\title{
Factors Influencing the Performance of Shared Services Centres
}

Thesis submitted in accordance with the requirements of the University of Liverpool for award of the degree of Doctor of Business Administration by Cicero Ferreira.

ORCID ID: 0000-0003-3334-2595

October 2016 


\begin{abstract}
The efficient use of public money is a concern of all society. The more efficient the government machinery, the smaller the portion of funds raised assigned to operating costs and more invested in health, education, security, and transport, for instance. Shared service centres (SSCs) have contributed to this, allowing billions of dollars cost cut in the public service in several countries. However, cases of SSCs failures are causing billiondollar losses, and it is necessary to understand and overcome the causes of failures. This scenery has motivated me to study the factors that contribute to the performance of SSCs and to explore whether there are SSCs models in the public sector that are simply copies of SSCs models of the private sector (without the necessary adaptations). Three objectives were established for the research: to analyse the factors that could influence the performance of SSC; to investigate to which extent there are significant differences between private and public SSCs; and to analyse if there are evidence of copy problems. Also, a principal research question: to what extent does factors such as culture, leadership, resources and readiness for change, influence on service excellence, market orientation and performance of Shared Services Centres? An action research design was defined with a mixed, quantitative and qualitative approach. The quantitative approach refers to a conceptual model with seven constructs (culture, leadership, resources, readiness for change, service excellence, market orientation, and performance), individually validated by previous studies. This proposed model was validated empirically through a survey with 146 SSCs respondents from countries like the USA, the UK, Canada, and Brazil, and the research hypotheses were confirmed. On the qualitative approach, were applied open-ended questions submitted later to content analysis, and the quantitative and qualitative results were discussed with an Action Learning Set
\end{abstract}


composed of SSC managers and public-sector experts. The main findings were the confirmation of the proposed model variables' relationship, influencing the SSC performance. This allows managers to establish actions to improve the similar dimensions of their SSC, improving the overall performance. It was also confirmed the existence of significant differences in the context of public SSCs operation regarding the private. These findings were also discussed in the Action Learning Set and resulted in eight measures proposed for the best adaptation of public SSC models to the reality of the public sector. For further research, I suggest investigating whether the SSCs of the public sector have in fact the minimum requirements to be classified as SSCs or are just departments that centralised services from other areas and were named SSC for convenience. Another opportunity for research is to verify to what extent the New Public Management has been successful in encouraging the adoption of SSCs, e.g. in countries like the UK and the US, so that they were more oriented to their clients, as this research found evidence there are public SSCs not oriented to their customers. 


\section{ACKNOWLEDGEMENTS}

Firstly, I would like to thank God, because without His help I would not have gotten this far. Thanks also to my mother, for their continuous prayers on my behalf and unrestricted support to my studies. Many other people were decisive and helped me throughout these years of walking during the doctorate, and I would like to take this opportunity to thank them. Dr. Allan Macpherson, for his meaningful and constructive comments, and all other DBA professors; to all my colleagues of DBA and the officials of the doctorate program. To the respondents of my survey, without which the results and contributions of this study would not exist, my many thanks. I also thank all the experts and managers that have integrated my Action Learning Set and, with their experience and dedication, helped me turn raw data into refined discoveries and actionable knowledge.

Thank you very much, everyone!

Cicero Ferreira 


\section{TABLE OF CONTENTS}

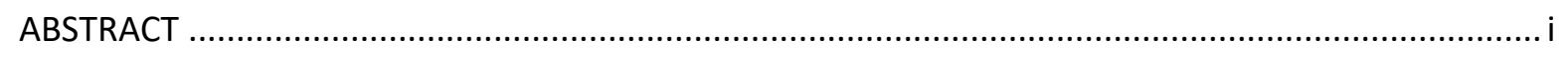

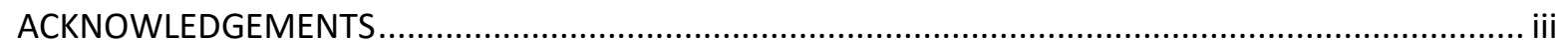

LIST OF FIGURES

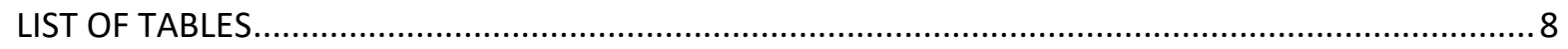

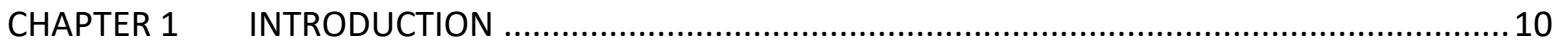

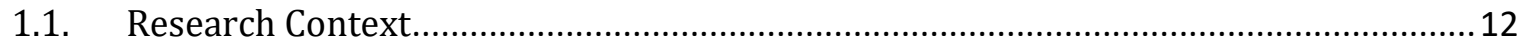

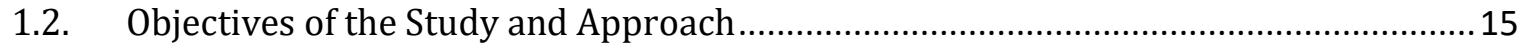

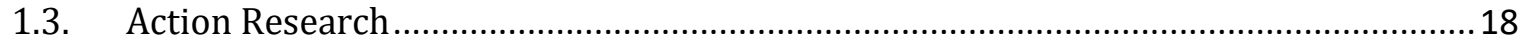

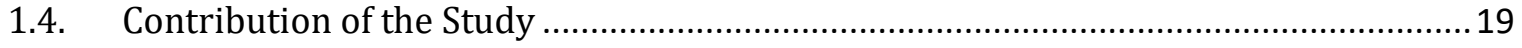

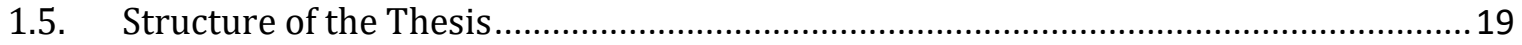

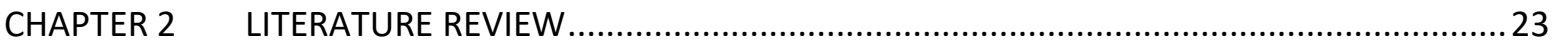

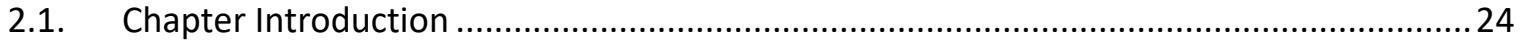

2.2. The Public Service Adoption of Private Sector Management Practices ............................. 26

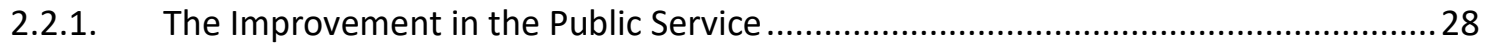

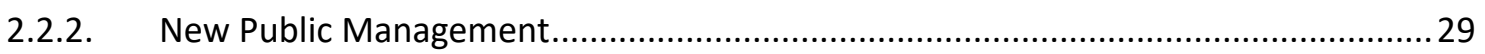

2.2.3. Key Differences Between the Private Sector and the Public Sector ........................... 31

2.3. Shared Services Centre: Definition and Genesis............................................................... 32

2.4. Shared Services Centre: Expansion in the Private Sector .............................................. 38

2.4.1. The Reported Problems with the Implementation of SSC in the Private Sector ........ 40

2.5. Shared Services Centre: Expansion in the Public Sector ............................................... 40

2.5.1. Expansion of the Shared Services Centre (SSC) in the public sector worldwide ........ 41

2.5.2. Example of Benefits - Expansion of SSC in Local Governments in the UK and Losses in

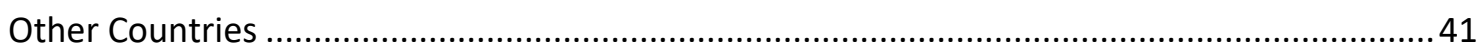

2.5.3. Example of Services Provided by the SSC in Local Governments .............................. 42

2.5.4. Three Cases to Illustrate Problems with Shared Services Centres .............................. 42

2.6. Conceptual Model: Modelling, Literature Review of each Concept and the Proposed Model 44

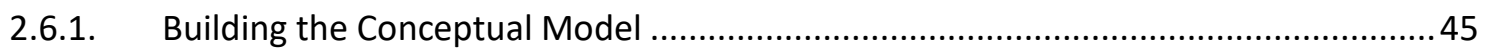

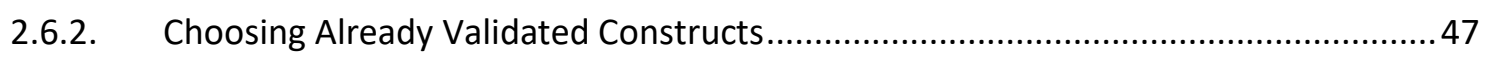

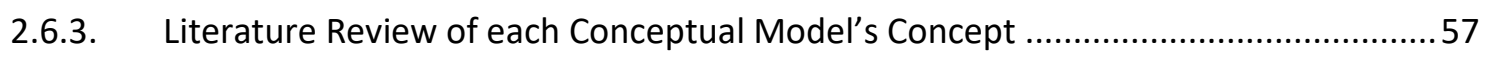

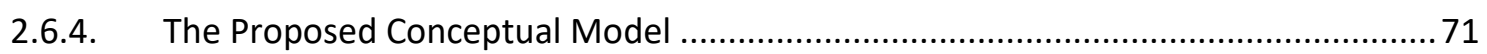

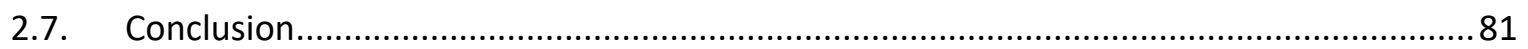




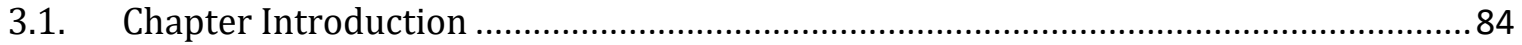

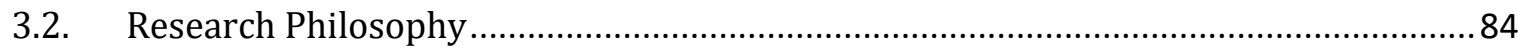

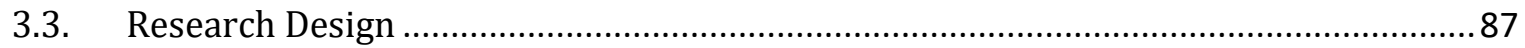

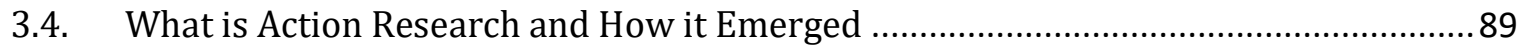

3.5. How this Study Applied the Action Research................................................................ 90

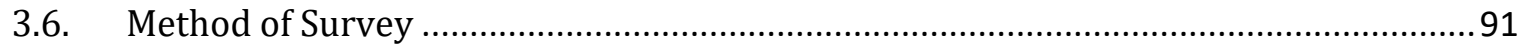

3.6.1. Sampling Approach and Considerations ................................................................... 91

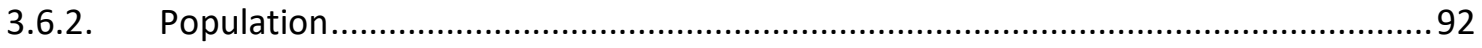

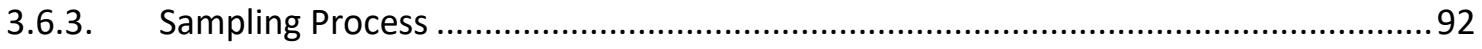

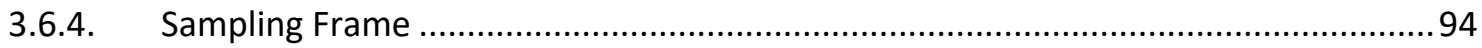

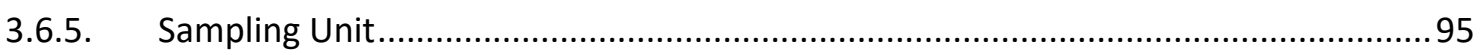

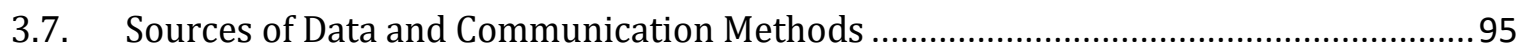

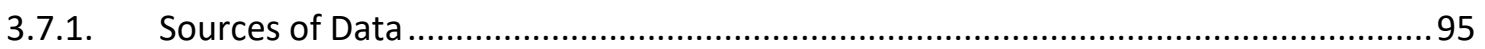

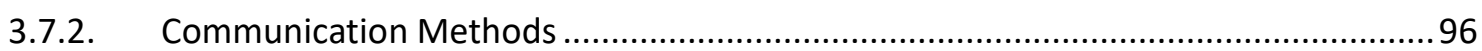

3.8. Questionnaire and Measurement Scales .................................................................. 97

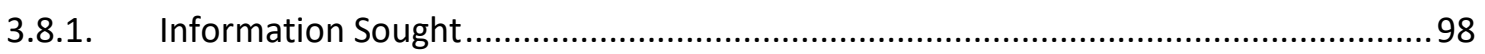

3.8.2. Type of Questionnaire and Method of Administration .............................................. 99

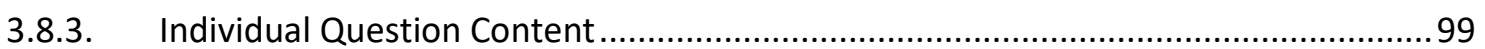

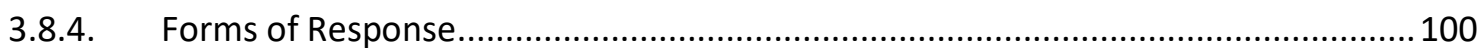

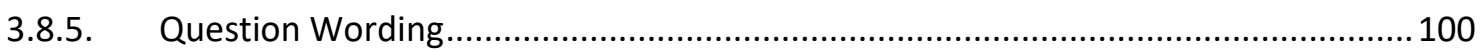

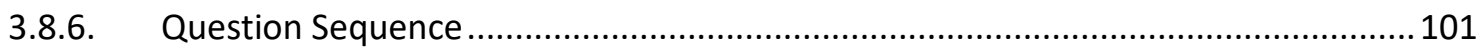

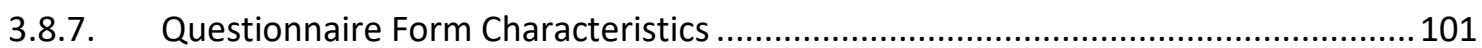

3.8.8. Re-examination and Revision of the Questionnaire ............................................. 102

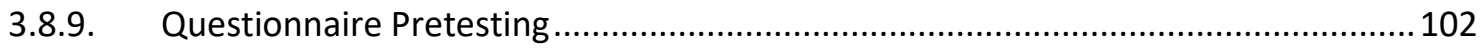

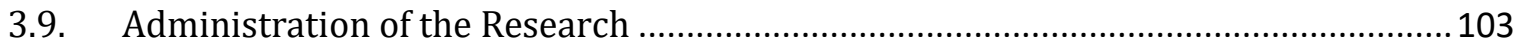

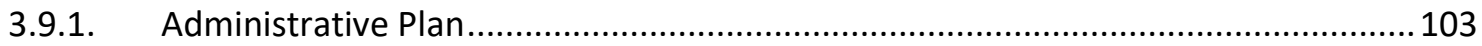

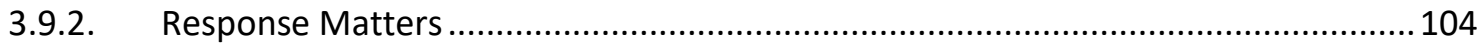

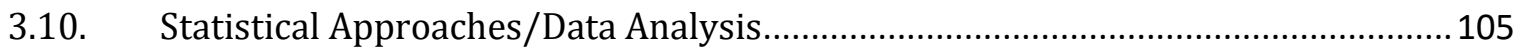

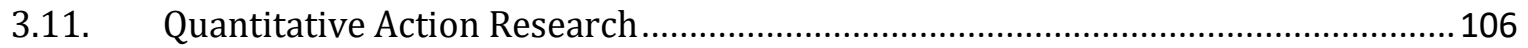

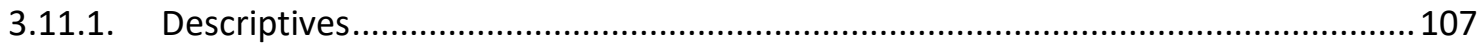

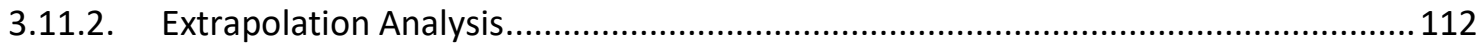

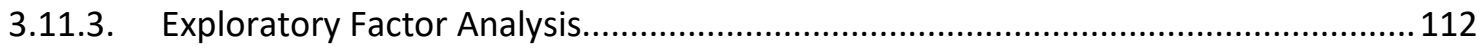

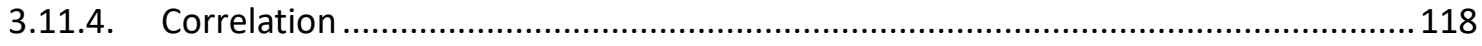

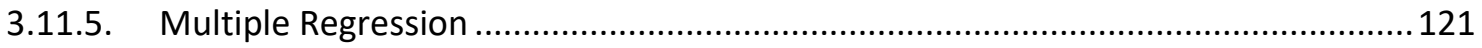




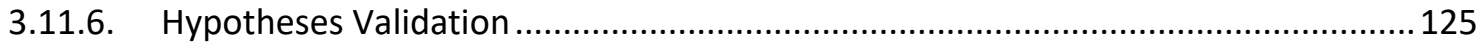

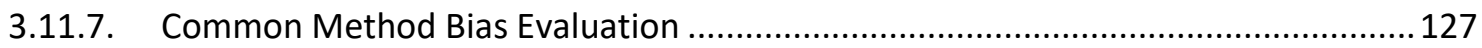

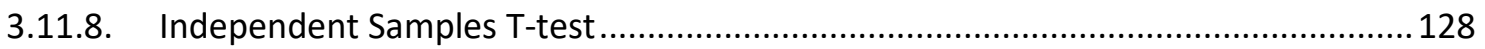

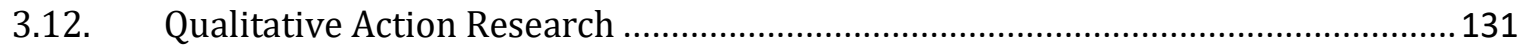

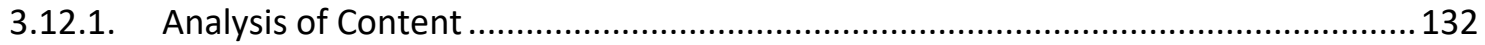

3.12.2. Results of the Analysis of Categories, Based on the Dimensions Impacted .............136

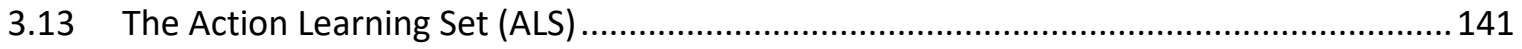

3.13.1 Composition of the Action Learning Set for this thesis .......................................141

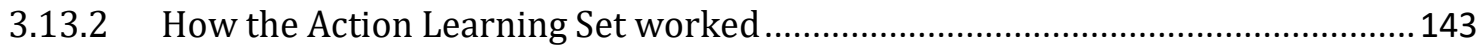

3.13.3 Dynamics and contributions of the Action Learning Set.....................................144

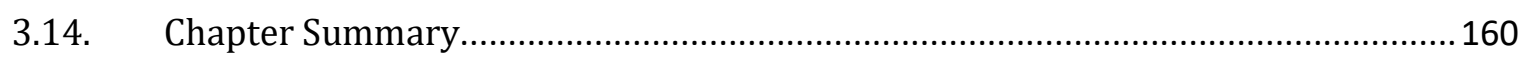

CHAPTER 4 CONCLUSIONS, IMPLICATIONS, AND RECOMMENDATIONS ..................................... 161

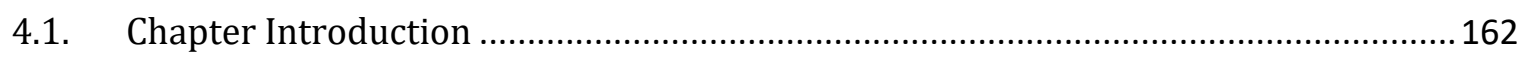

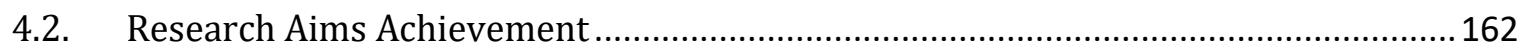

4.3. Discussion of the Results, Relevant Actions, and Recommendations for Public SSC

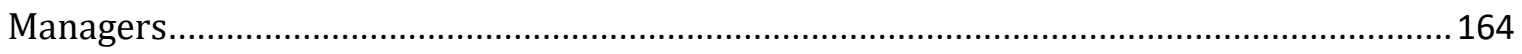

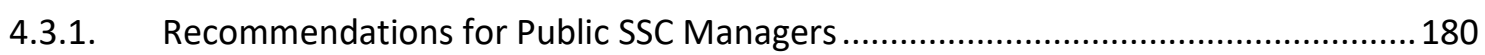

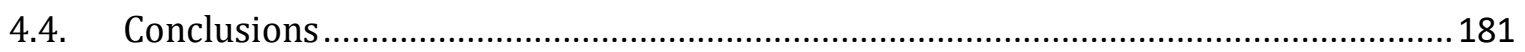

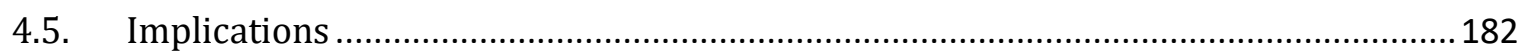

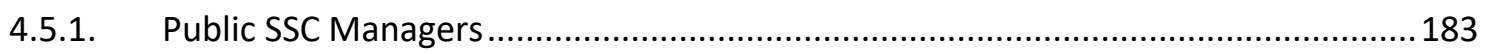

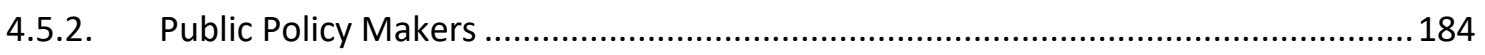

CHAPTER 5 REFLECTION AND LEARNING AS AN ACTION RESEARCHER ..................................... 185

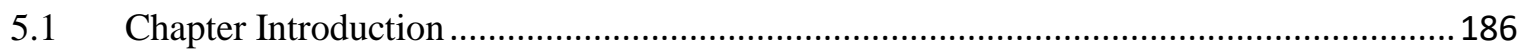

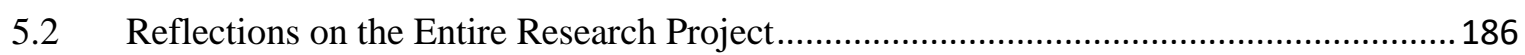

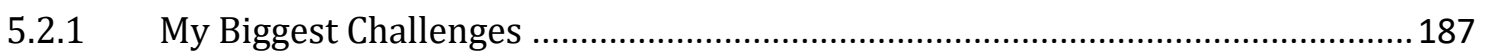

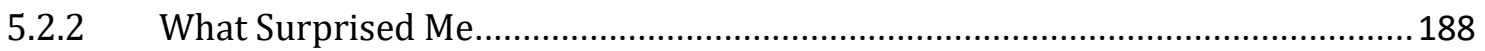

5.2.3 What Went Well and What Went Less Well ....................................................... 189

5.2.4 Personal Assumptions and Values Influencing the Research .............................189

5.3 Reflections on Each Step of the Research Project ......................................................... 190

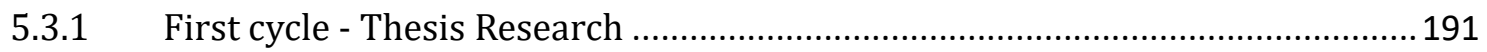

5.3.2 Second cycle - Survey, and Action Learning Set - ALS ......................................192

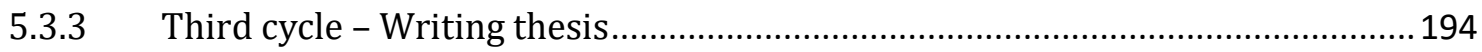

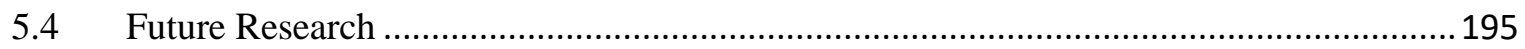

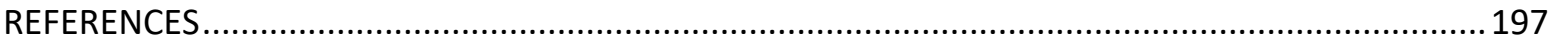

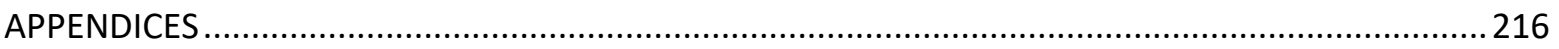

Appendix A - Examples of cases of problems with SSCs in the public or non-profit sector . 217 
Appendix B - EUSurvey Questionnaire

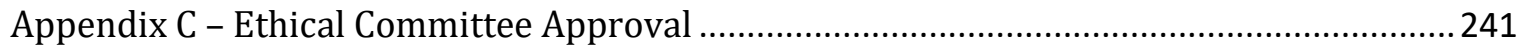

Appendix D - Example of Emails to the Action Learning Set.................................................2 242 


\section{LIST OF FIGURES}

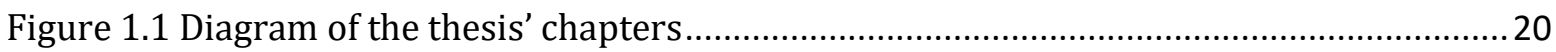

Figure 2.1 Conceptual model of factors influencing SSC performance ....................................... 74

Figure 3.1 Sampling Process adapted from Kalyan Acharjya (2016) ........................................ 93

Figure 3.2 Example of the Likert scale applied to the questionnaire ......................................... 100

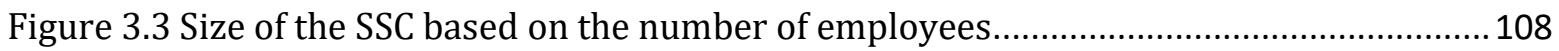

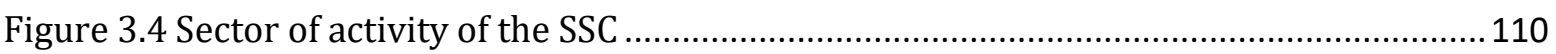

Figure 3.5 Conceptual model of factors influencing SSC performance, with the hypotheses... 126

Figure 3.6 Screen snapshot of the software SPSS Text Analytics for Surveys version 4.0 .......133

Figure 3.7 Schematic View of the Action Learning Set for this DBA Thesis ..............................142

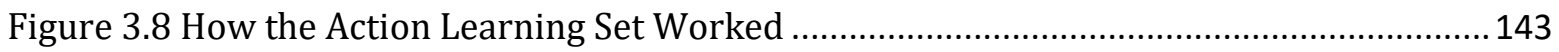

Figure 3.9 Self-reflection and learning roadmap based on (Coghlan \& Brannick, 2014)..........191

\section{LIST OF TABLES}

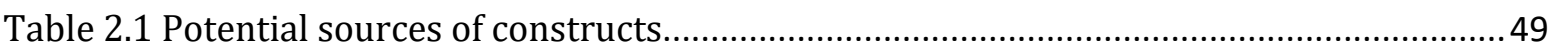

Table 2.2 Conceptual model variables and the corresponding support from the literature ...... 73

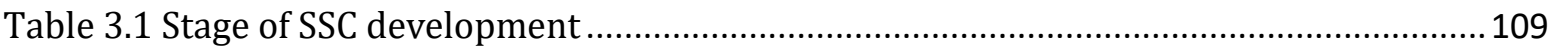

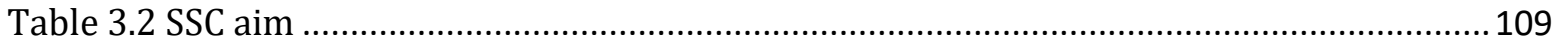

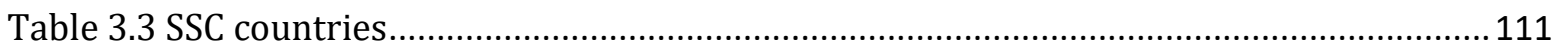

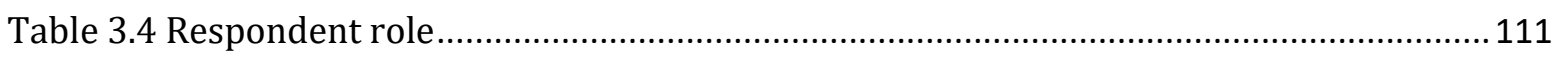

Table 3.5 Descriptive statistics of market orientation ............................................................116

Table 3.6 Exploratory factor analysis results - Rotated Component Matrix - Market Orientation

117

Table 3.19 Correlation Analysis of independent variables Culture, Leadership, Resources and

Readiness for Change, and the dependent variable Excellence...............................................120

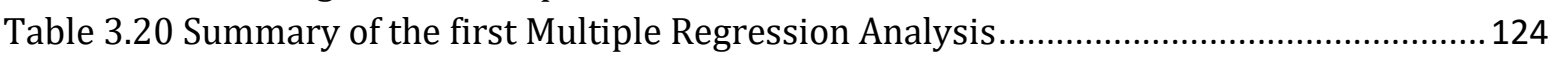

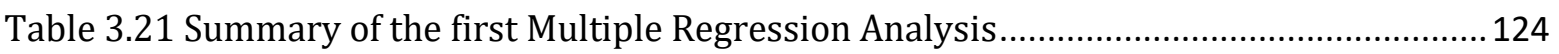

Table 3.22 Summary of the second Multiple Regression Analysis..........................................125

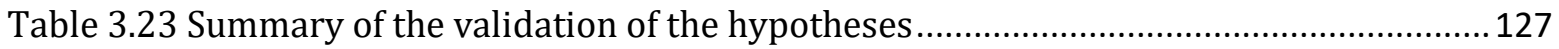

Table 3.24 PCA - Total Variance Explained - Harman's Test .......................................................... 128

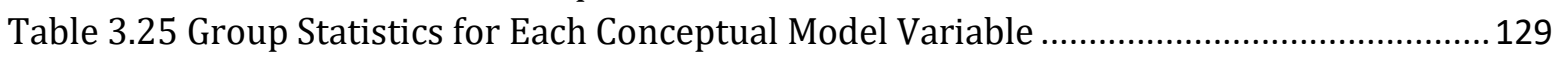

Table 3.26 Independent Samples Test (public versus private SSC) ............................................130

Table 3.27 Categorisation of responses during the analysis of content ..................................... 135

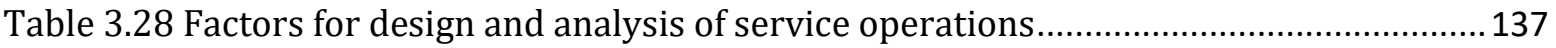

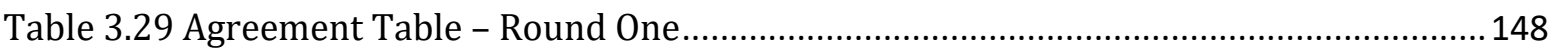

Table 3.30 ALS Members Agreeing with my Initial Interpretation of the Key Findings - Round

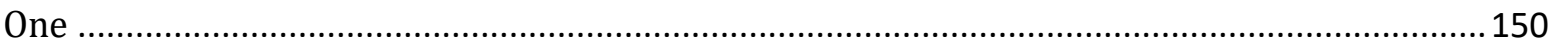

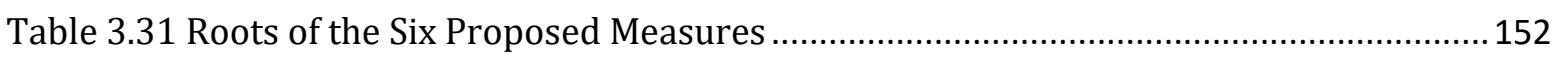

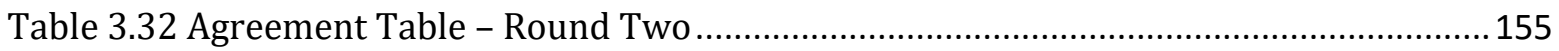

Table 3.33 ALS Members Agreeing with the Six Measures Proposed.........................................156

Table 4.1 Relevant actions to take the Measure 1 - Create its own service culture ...................170

Table 4.2 Relevant actions to take the Measure 2 - Steering by its customers (client focus) ..172 
Table 4.3 Relevant actions to take the Measure 3 - Develop an entrepreneurial leadership...173 Table 4.4 Relevant actions to take the Measure 4 - Bring some people from the industry to the public SSC.

Table 4.5 Relevant actions to take the Measure 5 - Public SSC as a State Policy .......................176

Table 4.6 Relevant actions to take the Measure 6 - Develop organisational resilience.............177

Table 4.7 Relevant actions to take the Measure 7 - Become 'market-oriented' ........................179

Table 4.8 Relevant actions to take the Measure 8 - Using the conceptual model within a diagnostic framework. 
CHAPTER 1

\section{INTRODUCTION}


Shared Services Centres (SSCs) have been shown to be a phenomenon with accelerated growth and represent an evolution of the traditional organisational models, more precisely, in support of service operations, such as HR, finance, purchasing, accounting and IT, as well as numerous others (Richter \& Brühl, 2016). An SSC can be defined as the concentration of a company's resources, for the processing of common and repetitive activities, to serve the internal partners with a high level of quality and reduced cost, to please customers, and to add value to the business (Schulman et al., 1999). Its most intense use began in the mid-1990 and is usually reported as pioneers in their adoption, GE, and Unilever. Today virtually every 500 largest companies listed by Fortune magazine have an SSC (Fersht \& Aird, 2011); this includes Walmart, Shell, Exxon Mobil, BP, and Toyota, to illustrate some. Even these few details are enough to explain the increasing adoption and relevance of SSC to the private sector. Another important information is that the gains from reduced operating costs in SSC implementation projects may exceed 20\% (Schulman et al., 1999; Quinn et al., 2000; Yusof et al., 2016). However, in the public sector, only a few countries like Australia, Canada, the USA and the UK, have been able to benefit in large-scale from the organisational model SSC, reducing their operational costs in billions of dollars and improving the quality of public service (OECD, 1995, 2010). That said, I will explain the process of choosing my research topic. The choice of a subject for social research is informed by the interest of the researcher, the influence of their environment, and the literature reviewed. In my case, these three components were present in the choice of the Shared Services Centre as the theme of my research. My research shows that there is a potential for SSC adoption in public sector of many countries, especially in developing countries, the case of Brazil, my country of birth and where I live. In Brazil, there are numerous SSCs in the private sector, but practically none in Government. 
The reason for the choice of the theme - In my professional career, as a management consultant, I worked for large management consulting firms such as Gemini Consulting, PricewaterhouseCoopers, and IBM Global Business Services. There, I had the opportunity to participate and lead numerous projects of diagnosis, design, and deployment of SSCs in the private sector. In my experience, when I did my Masters in business administration, I found during the research for the dissertation a lack of studies on SSC in the public segment. Also, the potential application of SSCs in public management and its capacity in the release of funds for governments core activities (better use of citizen's money on education, security, transportation, health, etc.) drew my attention to this opportunity for a contribution to the Academy and practitioners. Public managers, especially in developing countries, need and will need further systematised knowledge on this subject so that they can deploy SSCs effectively and without incurring basic errors that have led some public SSCs to failure with huge losses, like those in Australia found during the literature review for this research (NCA, 2015). This context described quickly, and the expansion scenario make the theme Shared Services Centre in the public sector very relevant and thought-provoking. In the following sections, I present more information about the context and approach of the research; the objectives of the study; the contributions of the study to the Academy, managers and SSC practitioners; and finally, I summarise what is presented in the remaining chapters of this thesis.

\subsection{Research Context}

As illustrated above, in the private sector, the SSCs have been adopted in virtually all economic sectors, most often by large enterprises. In these companies, the concentration of support services (such as HR, IT, finance, etc.) in a new service unit 
(SSC), conducted as a business and oriented to its customers, usually generate the greatest benefits, with substantial cost reduction and improving the quality of services provided by the SSC (Schwarz, 2014). Although for many years the SSCs are part of the public policy of some federal (e.g. the USA), state (e.g. Australia) and inter-municipal (e.g. the UK) governments, this occurs especially in developed countries. This fact is possibly due to greater professionalism in public administration and a lesser degree of political interference when changing governments, and better level of awareness of citizens about their rights and possibilities for improvement in public services (Denhardt \& Denhardt, 2000; Tomkinson, 2007). However, developing countries can also benefit from the implementation of SSCs in public administration, and I intend to contribute to this with this research. During the preliminary searches, I found that there is very little research on the SSCs of the public sector, as I report in the next chapter, the literature review. Based on these preliminary studies, including cases of failure of some public SSCs, e.g. NCA (2015), it became my initial assumption that some of the problems are related to two central issues: no market orientation and a copy of the SSC model of the industry, without proper adaptation. Being customer oriented (market-oriented) is a fundamental principle of an SSC, whether industry or the public sector (Schulman et al., 1999; Bergeron, 2002; Tomkinson, 2007). Thus, I decided to investigate what factors influence the performance of an SSC and how market orientation relates to this performance. For doing this, I prepared a conceptual model and tested it empirically. Regarding the copy of the SSC model that was not implemented properly (Ulbrich, 2010; Estabrooks et al., 2006), it was my assumption that this simple act of copying and pasting the private model of SSC did not consider the specific characteristics of the services rendered by the public sector, like the political context, nature and finality of the Civil Service-different from the private service-and the Civil Service staff profile. These 
differences may be more pronounced in a country and less in another, with a greater presence of best management practices observed by research in the public sector of developed countries.

Given these circumstances, I also decided to investigate in this research two groups of SSCs, public and private, especially in countries like the UK, the USA, Canada, and Brazil, to understand the main differences in the characteristics of services provided by public and private SSCs, and in their modus operandi, and results obtained by these groups in the scope of operation and management. I chose the UK, the USA and Canada, mainly for its tradition in applying SSC in the public sector, and Brazil because it is my home country and I intend to establish a knowledge foundation for future research and continue contributing to the advancement of its public SSCs. Besides the already mentioned studies, I investigated other areas of knowledge in management to gain insights and concepts for my research. For instance, the discussion on the appropriateness of the use of industry management practices in the public sector (Hughes, 2012; Pollitt, 1990; Flynn, 2007); the positive impacts of the use of SSCs in the public sector (Paagman et al., 2015; Goff, 2005; Janssen \& Joha, 2006); the reformist movement New Public Management (NPM), that defends the adoption of successful practices of the private enterprise in the Civil Service, and favoured the adoption of the SSCs by governments in countries like the USA and the UK (Hood, 1995b; Pollitt \& Summa, 1997); the comparisons between the practices of management in the public and private sectors (Hammer, 2007; Walsh et al., 2008; Denhardt \& Denhardt, 2007); and Knowledge Translation, concept that explains how to move knowledge (e.g. a shared services centre model) from one context (e.g. private sector) to another (e.g. public sector), 'translating it' accordingly (Armstrong et al., 2013; Straus et al., 2009; Ulbrich, 
2010). All these studies were also useful for a broader understanding of the phenomenon SSC and obtaining conceptual tools for analysing the research topic on the SSCs. Similarly, theories of the organisational model, management model, and service design were analysed, and concepts applicable to this research were systematically identified and compiled.

As for the ethical aspects of research, the methodological approach in all its aspects, especially the involvement of participants, was pre-approved by the Ethics Committee of the University of Liverpool. Although the participants' involvement was limited to providing answers to an online questionnaire for a non-controversial subject matter, all the initial contact to invite them, and clarifications on the nature of the research and its objectives, was provided within ethical standards for management research (Easterby-Smith et al., 2008). Furthermore, they were informed about the voluntariness of their involvement, and that they might interrupt such a participation at any time. Another important aspect highlighted was as for the confidentiality and anonymity of the individual answers, including on the non-disclosure of the name of their organisations. In addition to these precautions for ethics applied to the research, others have been taken to ensure complete adherence to the ten principles of ethics in social research to protect informants and research objects, according to Easterby-Smith et al. (2008, p.134).

\subsection{Objectives of the Study and Approach}

As discussed in the previous section, the SSC models introduced in the public sector were copied from the private sector, and the simple copy and paste may be the cause of problems that have led public SSCs to failure, since there are significant differences between the public and private management (see Section 2.2.3.), especially 
as for the context and nature. To understand the factors that influence the performance of SSCs and examine the differences empirically, I defined the following aims and research questions.

Research aims:

1. To analyse the factors that could influence the performance of SSC;

2. To investigate to which extent there are significant differences between private and public SSCs; and,

3. To analyse if there are evidence of copy problems (copy of SSC model from the industry right to the public sector without proper translation).

Based on the literature reviewed, I defined two research questions: RQ1: To what extent organisational factors (discussed below in this section) influence the performance of Shared Services Centres? RQ2: Do Shared Services Centres of the Public Sector differ from the SSCs of the Private Sector?

Research utilisation - Much has been discussed about the low level of practical application of the knowledge generated by the Academy. This subject is known as the 'theory-practice divide' (Lee \& Greenley, 2010; Halasz, 2016). For example, in some universities, the incentive model rewards the researchers based more on the amount of new knowledge generated than on the quality, or its effective contribution to improving management in the real world. It is a concern of this doctoral thesis to not only meet the requirements for obtaining the title of doctor in business administration by its author, but generate practical knowledge that is useful to managers, and others interested in the evolution of the SSCs management practices. For this, all the methodological approach was designed with this goal in mind, from the choice of the subject and setting 
of goals, until the formation of an Action Learning Set of experts in SSC and public service, to criticise the preliminary results of the analysis, help to broaden the understanding of the setting where the SSCs are inserted, and to generate knowledge that can be put into practice in SSCs.

Therefore, the research design was conceived to combine the methods of qualitative and quantitative traditions which, according to Creswell (2013), lead to complete results that can be directed to a practical application of the knowledge generated. The results are more complete because the limitations of a method are compensated by another. In the quantitative part of the inquiry, a conceptual model was conceived aiming to analyse the factors that influence the performance of the SSCs, composed by the variables Culture, Leadership, Resources, Readiness for Change, Excellence, Market Orientation, and Performance. The constructs and corresponding scales to these variables were obtained from previous empirical studies that had already validated these components of the conceptual model in other contexts. In the qualitative part of the model were studied the differences between the SSCs of the private and public sectors. In the collection of data for empirical validation of the conceptual model, the constructs and the corresponding scales were submitted through a questionnaire to managers and senior professionals of SSCs, resulting in a sample of 146 SSCs, public and private, establishing parameters for comparison. The qualitative part of the survey was applied using the same questionnaire with open-ended questions, whose answers were subjected to content analysis, and whose preliminary results were discussed with my Action Learning Set, consisting of executives from SSCs and public service experts. The Action Learning Set also evaluated and discussed the results of the quantitative part, and helped transform the knowledge generated by the research in eight measures, for 
adaptation and improvement, which can be implemented in the public sector SSCs to improve their performance.

\subsection{Action Research}

As my small management consulting company was not the proper setting for research, I chose the theme shared service centres in the public sector, as discussed in the previous sections. Then, the objectives established for the research mentioned above have led to a high degree of complexity for their achievement, requiring an approach that could reconcile quantitative studies (e.g. to understand the relationship of the factors that influence the performance of SSCs) and qualitative (e.g. to understand the differences in management of public and private sectors, or how the political influence affects the SSCs). I would also like, to promote change through the SSC managers. Therefore, I decided to adopt the approach Action Research (AR), in this case, aimed at solving the research problems and generating knowledge (Coghlan \& Brannick, 2014). The central idea of this approach is the use of scientific methods to study and solve organisational (or social) issues, together with people who experience such issues (Coghlan \& Brannick, 2014).

From the review of the literature on methodologies, I conceived a research design, based on AR, which could bring a solution to the issues in focus, and learning for me and the other participants. So, I could contribute with practitioners (e.g. managers of SSCs), the Academy and with my development and of those involved in the study. My role in the Action Learning Set (see details in Chapter 3) was as a researcher, change agent and facilitator in the consolidation of findings, knowledge, and actions for implementation. Throughout the research project, AR proved to be the right choice to deal with many 
methodological challenges, for instance, how to understand the social context of the civil SSCs, and how to put the knowledge developed in action.

\subsection{Contribution of the Study}

This study provides an original contribution to the development of theoretical and practical knowledge about the SSCs from the public sector and, equally important, the operational knowledge that is immediately applicable by SSCs public service managers. The knowledge proposed by this research can help in the adaptation and improvement of its operations to achieve client focus and high performance. The main contributions are the systematisation of the factors that influence the performance of an SSC (culture, leadership, resources, readiness for change, excellence, and market orientation), which enable managers to make the necessary adjustments for each factor and improve the performance of the SSC, as detailed in Section 4.3, Discussion of the Results. Also, eight measures that allow to adapt the current model of SSC, or implement a new one, as appropriate, to reflect the environment of the public sector and so that it is not a simple copy of the SSCs of the private sector. Furthermore, a contribution to the rescue of the history of the development of the SSC concepts and practices, as this research has identified as one of the forerunners of modern SSCs, an initiative of the US education sector in the 1960s, as discussed in Section 2.3, Shared Services Centre: Definition and Genesis.

\subsection{Structure of the Thesis}

This section provides an overview of this doctoral thesis, including an illustrative diagram (Figure 1.1 below) and a brief explanation of the purpose of each of its five chapters. 
Figure 1.1 Diagram of the thesis' chapters

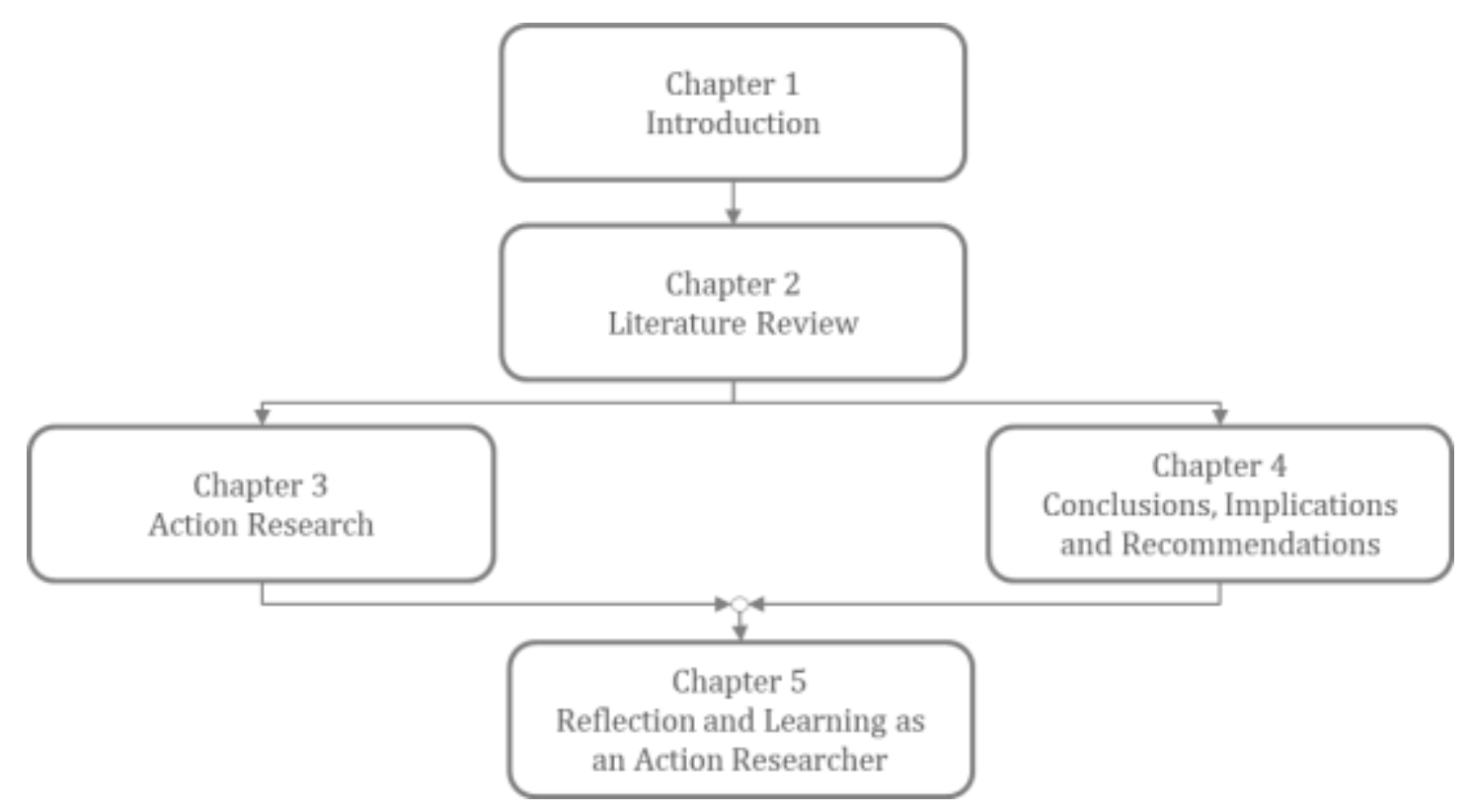

Chapter 1, Introduction, provides general information about the research project, the choice of the theme, its relevance, the background of the researcher, the objectives of the study, which methodological approaches were used, and the ethical aspects involved, allowing the reader a quick recognition of the content of the document. In addition to the introductory text, presents the Research Context, Objectives of the Study and Approach, Action Research, Contribution of the Study, and Structure of the Thesis.

Chapter 2, Literature Review, aims to present the knowledge identified and critically evaluated in my research on existing studies, on the topic Shared Services Centre, and concerning the concepts and models in management, mainly in the organisational and service operation fields, which I later used to investigate holistically the SSCs, target of this research. This chapter also informs the origin of the constructs and measurement scales used to build the conceptual framework of this research, which also led to the questionnaire used as an instrument for survey data collection. The previous studies that validate such constructs are properly identified and each of the 
seven components of the model (culture, leadership, resources, readiness for change, excellence, market orientation, and performance) is defined and discussed. The literature review was helpful throughout the project cycle; from the choice of the research problem, the better understanding of the public service, to the analysis and conclusions about the results.

Chapter 3, Action Research, aims to inform how the project was developed to solve specific problems of public sector SSCs, how knowledge was produced and how it was ensured that the knowledge generated by this research is applicable in practice of SSCs, with emphasis on the public sector. Thus, discusses approaches and methods used to conduct the research, explaining why the option was for a mixed method (qualitative and quantitative), and why it was necessary including an Action Learning Set formed by specialists in public service and shared services. Moreover, it describes the sources of data, how data were collected and analysed, and details about the questionnaire, survey controls, sample, participants, and the like. As for the quantitative action research approach, it presents the analysis and the results achieved, informing the sample characteristics and details of the statistical treatment applied to the sample both the validation and analysis (e.g. exploratory factor analysis for each construct, correlation, multiple-regression, hypotheses validation, among others). Therefore, it is in this chapter that are discussed the results of the empirical validation of the conceptual model proposed by this research. Also, it presents how the experience with the ALS contributed to my academic and professional growth and development.

Chapter 4, Conclusions, Implications, and Recommendations, is intended to report how the research made it possible to answer the research questions, and, so, achieve the goals established for the study. Thus, the main findings are discussed, and summarised 
the significance of this research. Moreover, there are presented the results of the validation of the hypotheses set out in the conceptual model. In addition, eight measures for the right adaptation of the SSC into the public service context are proposed, and some recommendations for SSC managers are made. Finally, are drawn up the implications of the knowledge generated by this research, both for Academia and the public sector SSCs.

Chapter 5, Reflection and Learning as an Action Researcher, shares my learning and development as an action researcher. Also, what has changed in my attitudes, assumptions and skills as a scholar-practitioner. Additionally, it gives suggestions to expand the public-sector SSC knowledge in future researches as were identified from this work. 
CHAPTER 2

LITERATURE REVIEW 


\subsection{Chapter Introduction}

This chapter discusses the literature reviewed to support the formulation of the research question, develop the conceptual model that directed the survey in the field, and perform the analyses and conclusions of the thesis.

Through literature review, it was possible to identify what other researchers have researched on the shared service centres (SSC) and find that there are very few academic works on public sector SSC, that, as explained in Chapter 1, intended to be the main beneficiary of this thesis. To give an idea on how the SSC is little explored, I searched the engine Discovery from the University of Liverpool for the keyword "shared services" in 30/10/16 ("shared services" with quotation marks, present in titles, and only in academic journals) and resulted in 396 texts; of which only 14 mentioned the term "public service" (also searched with quotation marks) in some part of the text. (Discover accesses the main scientific knowledge bases via EbscoHost). These few articles found have sought to study, for example, the following phenomena related to the Public Sector SSC: organisation redesign (Wang et al., 2007), case study (Becker et al., 2009; Ulbrich, 2010), critical success factors for adopting SSC (Borman \& Janssen, 2012), motives for introducing SSC (Paagman et al., 2015), SSC implementation (Farquhar et al., 2006), IT governance and SSC (Janssen \& Joha, 2007), among others. There was no research found seeking to understand the factors influencing the performance of a shared services centre, and this motivated me to perform this research. Another fact that caught my attention is that, though the SSC in the public sector has been born under the mantle of the New Public Management (which seeks, among other things, better serve interests of citizens), there are indications in the literature that the quality of service might not have improved with SSC, and the public SSC may not be 
market-oriented (Pollitt \& Summa, 1997; Tomkinson, 2007). Moreover, these articles also helped me find new references to other studies that complemented my knowledge on the subject. Similarly, my participation in two international conferences on SSC in the public sector, in the USA (Washington DC and Chicago), also broadened my vision and understanding of the topic.

In reviewing the previous studies, the gaps were evident, and this made it possible for me to choose to research an area not yet explored, that is the factors that can influence the performance of SSCs. To gain a broader understanding of what factors affect the SSC performance I tried to understand the drivers for SSC, and the SSC as a service operation, besides the factors that can influence its performance. Also, I tried to locate the SSC within our understanding of organisational research. All this context mentioned contributed to addressing and answering the two research questions, discussed in Section 1.2, RQ1: To what extent organisational factors influence the performance of Shared Services Centres? RQ2: Do Shared Services Centres of the Public Sector differ from the SSCs of the Industry?

Besides this introduction, this chapter covers the following content: first (Section 2.2), discusses the adoption of management practices from the industry by the public sector, exploring how it occurs and analysing some theories and concepts involved, for example, the New Public Management. These discussions are particularly important for understanding how the public sector 'imported' the management practice 'SSC' from the industry. Afterwards (Section 2.3), the SSCs are defined and the origins of this organisational model discussed, including for what purposes the companies and governments use them as service operations, serving internal and external customers. Then (Section 2.4), it explores the trajectory of expansion of SSCs in the corporate world, 
starting from the reasons that led the industry to embrace the SSCs with so much emphasis, and the associated risks are identified in this new organisational paradigm. In the next section (2.5), it focuses the expansion of the SSCs in the public sector, the reasons that led to its expansion, and the tendencies of growth observed for the use of SSCs for local and national governments. It goes on then to the exploration of the elements that can impact the SSC performance, as an operation of services, through the discussion of the factors that can influence its performance (Section 2.6), passing by the formulation of the conceptual model and hypotheses of inquiry. Finally, the Conclusion section (2.7) summarises how the issues raised in the discussions of this chapter were addressed in my research and offered subsidies to answer the research questions.

\subsection{The Public Service Adoption of Private Sector Management Practices}

As this thesis approaches with emphasis the public sector's Shared Services Centre, it is useful to delineate the public service context where such SSC have emerged. The term 'service' can be understood from different perspectives, such as from the supplier of its execution as well as from the benefits generated and the customer experience. As the investigation of my thesis is related to the performance of SSC, associated with the way in which the services are provided, an operational definition of the view of the service execution seemed the most appropriate, and the definition of Grönroos (2011, p. 285) was chosen:

"Service is value-creating support to another party's practices... this support may either relieve customers from taking on some task or enable them to do something that otherwise would not be possible to accomplish or would be accomplished less efficiently or effectively (Grönroos, 2011, p. 285)." 
A standard definition of 'public service' in academic texts was not found. To have a working definition, the Merriam-Webster dictionary entry was adopted:

"The business of supplying something (such as electricity, gas, or transportation) to the members of a community; work that someone does as part of a government: the work done by public servants (Merriam-Webster, 2015b)."

A simple reading of the definitions of 'service' and 'public service' makes it clear that there may be similarities between the private and the public service. Nevertheless, there is an old discussion about the appropriateness of using successful management practices of private management, in the public sector (Hughes, 2012; Pollitt, 1990; Allison, 1992). Authors who are against the use of private management practices in public service say, for example, that the public sector is so specific that private sector models become irrelevant to its operation. They also argue that it is very difficult to establish objectives in the public sector, as well as keeping them as a target for some time because they change constantly; and if the objectives change frequently, other industry practices will no longer make sense. Another relevant difficulty would be measuring results in the public sector (Hughes, 2012; Pollitt, 1990; Flynn, 2007). However, these authors recognise that it is necessary for the public sector to be effective in cost and manage performance, but, with exclusive management practices. The researchers who advocate the use of private management practices in public service have argued, for instance, that the industry is inherently more efficient by having practices already tested by time, and the public-sector environment leads to inefficient management practice. They mention, as an example, that similar contracts are much more expensive in the public sector (Hughes, 2012). Regardless of the conceptual discussion, it is certain that more and more the practices of private management are 
being placed in the public service, and SSCs were no exception, as we have seen so far and we will see in the following subsections.

\subsubsection{The Improvement in the Public Service}

Since I discuss public service operations in this thesis, it is useful to seek to understand how studies already performed, approached improvement in the public service. Boyne (2003, p. 223) proposed a definition of public service improvement based on quality: "A closer correspondence between the perception of the real, and wanted, patterns for the public service"; in other words, the more the real performance to be brought near of the wanted, the better. The question arises, however, as to the use of the word perception; for example, an improvement from whose perspective, as the public service has by nature many stakeholders, such as citizens, civil servants, the Government, the control bodies, and so forth. In the SSC context, there are also SSCs providing services for other agencies or departments of the Government and not solely to the citizen (this is important when considering the conceptual model set to explore the problem addressed by this research). An alternative view to improving the public service is adopted by other authors who focus on the customer's perception. According to Osborne (2010), to mention one of this authors, service performance is subjective and related to the client expectation and their perception of the quality of the process that provides the service.

Improving the quality and the performance of the public service is a continuous demand. The public sector is susceptible to changes in the local economy in such a way that during periods of lower tax revenue, there is more pressure to cut operating costs to release money for investments (Gershon, 2004; Farquhar et al., 2006). This pressure creates a demand for innovations in service operations of Governments (Gershon, 2004; 
Zuniga \& Murillo, 2014), and it was in a similar context that the SSC arose as a Government policy in the USA and the United Kingdom, as part of the New Public Management movement (Gershon, 2004; Schwarz, 2014; Tomkinson, 2007), addressed below. The SSCs were an appropriate response since they enable significant reductions in costs and improvements in the quality of services (Janssen \& Joha, 2006; Schwarz, 2014; Schulz \& Brenner, 2010).

\subsubsection{New Public Management}

The New Public Management (NPM) is a reformist movement with root in the 1970s/1980s, in Europe, with the intention of introducing the successful practices of private management into public administration (Hood, 1995b; Pollitt \& Summa, 1997). The primary goal was to provide better services to the public by encouraging the public administration to utilise their limited resources more efficiently. The term management was used instead of administration to underline the need for public managers to be more pro-active (less bureaucratic in comparison with the old public administration) in conducting the public's affairs (Schwarz, 2014; Hood, 1995a). Among its key features we find: (a) the empowerment and the incentives given to managers to encourage greater efficiency; (b) the citizen-orientation; the citizen should be called customer and be treated like a client that is referred to as a client and must be treated as such; (c) planned and structured performance management; and (d) the systematic recruitment of third parties to provide certain public services (Denhardt \& Denhardt, 2000; Schwarz, 2014).

While the former public administration (old public administration) was anchored in the bureaucratic model and political theory, the NPM is based on the economic theory and a post-bureaucratic paradigm (Kapucu, 2006; Klijn, 2008; Said \& Thuraya, 2016). The public management began to assert itself as a catalytic agent of market forces, 
shunning the role of a policy implementer, responsible for isolated goals dictated by politicians politically (Kapucu, 2006; Osborne, 1993). Thus, the public administration should not produce its goods and services, but provide goods and services that are produced by others, and regulated by it. The New Public Management has paved the way for the use of private resources, non-profit organisations, and innovative structures (such as decentralisation models and the SSC), as well as better technology in the provision of public services (Kapucu, 2006; Osborne, 1993). Additionally, it promoted an entrepreneurial culture with a focus on the reduction of costs and greater efficiency (Dunleavy \& Hood, 1994; Pesch, 2008). Critics of NPM say, for example, that there is no convergence among researchers about what is NPM (Hood \& Peters, 2013), and that using the word "client" is a mistake because citizens are much more than just recipients of services, they are voters and funders of public services (Kincaid, 1997).

With this background in mind, insight can be gained from the implicit and explicit principles that guided the design of public SSC, as lower operating costs, focus on results and outcomes (citizens), professional management, etc. (Osborne, 2010; Paagman et al., 2015). This research emphasises the focus on results and outcomes of the SSC, that is, its "market orientation (MO)" because the historical analysis reveals that the SSCs were born with this MO principle in their DNA. On the other hand, taking a model from the private sector and deploying it in the public sector requires some care because of the differences between these sectors, as discussed below.

Therefore, we have seen here that the NPM movement has encouraged governments to copy successful management models from the industry, and that this has occurred in the case of SSCs. However, there are significant differences between the contexts of private and the public sectors, as discussed below. These differences should 
have been considered for a correct translation (Armstrong et al., 2013; Straus et al., 2009; Ulbrich, 2010) of the industry's SSC model to be applied in the public sector, and this research verifies if there is evidence of this type of copy problem.

\subsubsection{Key Differences Between the Private Sector and the Public Sector}

Later in this study, I argue that the differences between the management context of the industry and the public sector justify the existence of variances in SSC models in these sectors, which often do not occur. Therefore, it is important to understand the main differences here in these two contexts. Several studies point to a significant difference in the essence and form of private sector management in comparison to the public sector. In the examples given below, some differences are identified that distinguish the public and private sector. In general, the surveyed authors present their arguments about the differences between management in both sectors in a convergent perspective. In a characterisation of the public sector, several features are often highlighted (Boyne, 2002):

- It is highly bureaucratic;

- Public managers are less materialistic and have a weaker organisational commitment;

- Organisations that receive funding from political sponsors are loyal to the preferences of these authorities at the expense of service users;

- The public sector has multiple sources of potentially conflicting authority;

- A discrepancy between the public service objectives and the actual outputs produced; private managers have more control over their organisations as opposed to public managers;

- Public organisations are funded by taxation rather than fees; 
- Other distinctions: Absence of competitive pressures; an overly formal structure for decision making; preoccupation with rules rather than results; and managers have less freedom to react to the circumstances they face (fragmentation of authority);

- Lastly, a characteristic worth highlighting is that the public sector is mainly concerned with working on the inputs, rules, and the process while the industry is more concerned with outputs and outcomes.

This research discusses the limitations of current models of public sector SSC, arising from an incomplete adaptation of these models when transferred from the private sector. Therefore, it is important to understand the significant differences between the two sectors, which elicit in a need for such adaptations. To give another example of those differences, Pollitt (1990) argues that it is foolhardy to import a foreign body of private management to the public sector because the ideology is different from the ideology of public service.

\subsection{Shared Services Centre: Definition and Genesis}

This section provides the definitions of SSC more used by researchers and seeks to bring more light to the roots of its emergence. Many authors report that SSC has raised in the 1990s (e.g. Tomkinson, 2007; Schulman et al., 1999), but my research found there is evidence the very first concepts of SSC has been born much earlier, in the 1960s, as demonstrated in this section. To complement, the view of critics and opponents of the SSC model is discussed.

Definitions of Shared Services Centre were initially conceived for the industry and varied in scope and emphasis. The following are five significant conceptions of SSC. The 
first, characterises the SSC as the concentration of a company's resources, for the processing of common and repetitive activities, to serve the internal partners with a high level of quality and low cost, to please customers, and to add value to the business (Schulman et al., 1999). The second conception, states that the SSC is created because the business units prefer to share a set of services, rather than to have them duplicated within the same organisation (Quinn et al., 2000). The third delineation defines the SSC as a cooperative strategy in which a subgroup of existing functions is concentrated into a new semi-autonomous business unit. The envisioned outcome of this project is greater efficiency, generation of value, reducing cost and a better service to internal customers of the organisation, competing in the market (Bergeron, 2002). In another definition, the SSC is a model of organisation in which a common company merges its business support functions - originally performed by various entities - operating in a separate unit that provides services to the rest of the company considering them as business customers (Ulrich, 1995). Finally, the fifth definition states that SSC is an organisational concept that consolidates the processes within the company group to reduce redundancies, and offers support processes; the definition goes on to list several defining characteristics of an SSC. It is a separate organisational unit in the group; it is aligned with the best practices of foreign competitors; it has the cost-cutting as a major driver of its adoption; is focused on internal clients; and is operated as a business (Schulz \& Brenner, 2010). Each of these definitions presents a different look at the same phenomenon, as emphasised by me underlining the keywords, which in my view are complementary.

The Shared Services Centre is, therefore, the type of high-performance organisation that sets up an operation based on a customer-supplier relationship, with a formal contract for service delivery and billing for the services delivered. This 
operation has its unique governance defined and driven by their customers who, besides sharing resources and investments in the SSC, actively engage in its decision-making process (Schulman et al., 1999; Quinn et al., 2000). For the SSC to work properly, these features exemplified here together with others, varying according to the degree of maturity of the organisation, must be present in the operation. Thus, the shared services unit needs to be semi-autonomous, not responding to any of their clients hierarchically, even though these customers take part collectively in its governance (Schulman et al., 1999). The SSC must seek continuous improvement in line with recognised market practices, aiming for excellence in services in its portfolio (Bangemann, 2005). According to Schulman et al. (1999), the SSC should conduct itself as a business unit and charge for its services. Finally, it must operate with a high degree of automation so that the goals of reducing costs and raising the quality of services can be achieved (Schwarz, 2014). All these characteristics need to be present so that an operation of services is considered a SSC.

If we want to draw a regressive timeline for establishing the genesis of the SSC, we can consider, on a historical basis, the milestones referred below, corresponding to three decades: A) the 1990s; B) the 1980s and C) the 1960s.

A) The 1990s. While there is no general agreement on the exact emergence date of the SSC, it is accepted that SSC has its growth in the private sector, in the 1990s for activities such as HR, IT, finance, and accounting, among others (e.g. Tomkinson, 2007; Schulman et al., 1999). These authors, among many others, refer the emergence of SSC in the Industry in the 1990s. Just to recall, regarding organisational design, in the late 1980s and 1990s, there was a predominance among corporations of decentralised Strategic Business Units. The reduction of more than $30 \%$ in costs and the impressive 
improvement in service quality increased the appeal of this new organisational model, with many companies preferring it over the centralised and decentralised models (Schulman et al., 1999; Ulrich, 1995). The significance of the emergence of SSC is that it may well signify a completely new paradigm in organisational design (Ulrich, 1995).

B) The 1980s. There is evidence the pioneering practices that led to the contemporary SSC concepts have their origins in practices that precede those just mentioned above. In 1984/1986 General Electric (GE) formed the 'Client Business Services', which operated shared financial and accounting services. Several publications have determined this to be the beginning of the current SSC (Quinn et al., 2000; Lacity \& Fox, 2008; Paagman et al., 2015). It is equally important to recall that in 1985 the concept of sharing also was present in what Porter (1985) called the horizontal organisation, i.e. arising opportunities between business units to share activities and resources of the value chain end and support activities, looking for economies of scale and scope. The purpose of this sharing can be related to cost reduction and an effort of differentiation. In the Porter's view, every company is a collection of activities performed to design, produce, market, deliver and support its product [or service] as part of its value chain, reflecting its history and business strategy. To share a valuable activity will turn in a significant advantage of costs if it involves a significant fraction of the assets or operating costs, and if the share to reduce the cost of its implementation. Porter also addresses the difficulties in establishing these interrelations. The sources of impediment mentioned by him are: the asymmetric benefits; the loss of autonomy and control; biased incentives; the different circumstances of the business units; and the fear of dealing improperly with the decentralisation (Porter, 1985). Also, being according to the referred author for the sharing possibilities are explored, it is necessary to establish 
a horizontal organisation, composed of mechanisms that enable the interrelationships between business units. An older reference to SSC is cited in the report of Alt \& Smits (2007), dating the SSC to a core banking system developed in Switzerland in 1973. However, it is not clear whether this was simply a consortium of banks or in fact an embryonic SSC.

C) The 1960s. During the research for this thesis, I discovered that during the 1960s, American researchers had been exploring the sharing of educational resources in rural areas (to improve the standard of schooling) while maintaining district autonomy. These organisations were termed 'Rural Shared Service' (DeBlassie \& Ludeman, 1973; Wilson, 1970). The main features of the educational initiative correlate with the SSC model, these include, providing services for multiple clients, sharing resources and governance, maintaining customer autonomy, and improving client satisfaction through better quality services. This discovery could set a new milestone in the formal reporting of the emergence of SSC. Nevertheless, it is important to note that Schwarz (2014) identified several European organisations operating in the 1930s with features of centralised support services to business units, for example, the Central Administrative Area in 1934 and the Central Department in 1936. However, further study is required to determine whether these initiatives can be classified as precursors to the SSC. The findings herein show that the root of the emergence of SSCs is much older than the 1990s, usually reported in studies on SSC as the time when the SSCs emerged (Schulman et al., 1999; Bergeron, 2002; Bangemann, 2005).

The SSC management model can be seen as successful, if we consider that most of the leading companies in the world adopt it (Fersht \& Aird, 2011), as well as most governments in developed countries, for instance, United States, United Kingdom, 
France, Italy, Spain, and Canada, among others. However, there are some critics of the SSC model (few as far as I could find in my research), that here are represented by Seddon (2008), perhaps the best known of them. Seddon argues that the SSC projects fail to achieve their goals by causing a disruption to the service flow when centralising these services, creating additional handoff, which generates more waste. Moreover, they cause, in his opinion, duplication of activities and rework increasing the time of delivery of the service to a client and creating what he calls "failure demand," which would be additional demands by customers due to failures and mistakes made in the first service (Seddon, 2008). The tone of his book "Systems thinking in the public sector: the failure of the reform regime... and a manifesto for a better way" seems more a political position against the British reform movement, which encouraged the adoption of SSC in public service, than an argument with empirical reasoning technique based on research. For example, he says that SSC is based in command-and-control and suffering from a systemic view, which in this way generalised is not true. There may be some SSC that way, but those that were implemented according to the concepts and best practices of SSC, are not (Schwarz, 2014; Tomkinson, 2007; Quinn et al., 2000). They excel at work toward the vision of end-to-end processes, considering the systemic vision and the work through cross-functional teams, where applicable (Brocke \& Rosemann, 2014; Schulman et al., 1999; Quinn et al., 2000). Furthermore, in his book, it is evident that Seddon (2008) uses the discussion of SSC, among other organisational practices, to defend the methodology created by him in the 1980s (The Vanguard Method). This methodology aims to help service providers to migrate from command-and-control to a more systemic approach, which is good, but inherent in the SSC model in the design of 
virtually all the SSC researches analysed (e.g. Schulz \& Brenner, 2010; Su, 2009; Bangemann, 2005; Schulman et al., 1999).

\subsection{Shared Services Centre: Expansion in the Private Sector}

In the previous section, I discussed the timeline of the emergence of the SSC. Here, I discuss the most relevant aspects of its expansion in the private sector. Until the 1970s, large corporations used the centralised model of organisation, derived from classical models of Taylor and Fayol, mainly for its support functions such as human resources, finance, information technology and accounting. The strengths of the centralised model are common systems, standards, consistent controls and economies of scale; by its turn, the weaknesses would be the passivity concerning business needs, the corporate support costs are not controlled by the business areas, and inflexibility on the dynamism of these areas, which are not seen as clients (Schulman et al., 1999).

In the 1980s, the strategic business units (SBU) emerged with greater emphasis. Each SBU had the freedom to get organised to reach the objectives that were attributed to it and so, they privileged the use of the decentralised model for the support functions (Porter, 1985). Because of this decentralisation emerged important redundancy of structures, resources, and activities. The main strengths referred to the decentralised model are the control by the SBUs of support services, and responsiveness to client's needs. As weaknesses stand out, the difficulty of adopting standards in the whole enterprise, the emergence of various types of controls, duplicating efforts, and the high costs caused by redundancy (Porter, 1985; Schulman et al., 1999).

It was in this context of decentralisation, and in search of better competitive conditions, that companies like Hewlett-Packard and General Electric (GE), referred to 
in the previous section, faced the reality of having high redundancy in its support functions. That circumstance led them to develop the model now known as Shared Services Centre (Mechling \& Schwarz, 2007; Schulman et al., 1999). Thereby, have become references as the first private sector companies to use this new model of service operation. The SSC model, which sought to combine the strengths of centralised and decentralised models, had high acceptance by businesses and began to be applied as an operational strategy in the industry, more intensely from the second half of the 1990s.

With the impact of successful cases of improving service quality and cost savings accounts of up to $30 \%$ for a significant number of companies, the use of SSC in the private sector has spread to virtually all industries (Mechling \& Schwarz, 2007; Quinn et al., 2000; Bergeron, 2002). To illustrate the expansion of SSC, let's look at some numbers for 2001, 2007 and 2011. In research conducted by Bywater in 2001 to the International Quality and Productivity Centre, with 200 of the 500 largest companies listed by Fortune magazine, it was found that $97 \%$ of them already used or intended to use SSC (Bywater, 2001). In 2007, research conducted by IBM Global Business Services, in cooperation with the Wharton School and The Economist Intelligence Unit, with more than 1200 CFOs and senior finance professionals from large companies around the world, revealed that more than $50 \%$ of the respondents have already adopted the SSC as effective practice in their organisations (Rogers et al., 2007). More recently, in 2011, it was found that about $90 \%$ of the companies present in the Fortune 500 had SSC (Fersht \& Aird, 2011), and it is reasonable to consider, roughly, that the SSC solution is present on most major global corporations. 


\subsubsection{Types of Problems with the Implementation of SSC in the Private Sector}

The expansion of the SSC also brought some problems worth to be known for perfecting the model. The decision and deployment of SSC involve risks and issues that must be well managed throughout the project lifecycle and service operation. One can assess the potential SSC risks through the model developed by Janssen \& Joha (2008), which provides analysis of the following potential risks for the implementation and operation of an SSC: service requirements not clear or unstable; uncertain interfaces, lack of participation of stakeholders; low quality on subcontracting; loss of control of the services; delays in the supply of services; low quality of services; increases in the costs of services; lack of motivation of service partners; services not accepted by clients; unpredictable costs for clients; detachment of goals and lack of governance mechanisms. Other risks are also reported for other studies, such as loss of control over the implementation costs (Bridelli, Werneck \& Martins, 2004). Expenditure on infrastructure, employee relocation, recruitment, training, technology, computer, travel and legal expenses can be easily underestimated and hardly controlled. For Bergeron (2002), among the problems for implementing an SSC are the laborious cultural change required to adopt a service to client vision, and the initial expenditure, which can be high, if compared, for example, with the outsourcing model, where the service provider assumes the inaugural investments. Therefore, challenging budgets, well-defined process indicators and a mentality of detailed control of costs are basic so that these problems are minimised (Deloitte, 2007).

\subsection{Shared Services Centre: Expansion in the Public Sector}

The Shared Services Centre (SSC) is a growing phenomenon in the public sector of many countries, promising to reduce costs and improve the quality of service to citizens (Tomkinson, 
2007). Since the 1990s they are part of strategies of different spheres of governments such as the US, Canada, the UK, and Australia, saving for the public sector of each country billions of dollars because of reduced operating costs (OECD, 1995, 2010). With few exceptions (e.g. the UK and the US), it appears more mature and widespread in the industry (Searle, 2014), which has been present since the early 1990s (Schulman et al., 1999).

\subsubsection{Expansion of the Shared Services Centre (SSC) in the public sector worldwide}

The OECD (Organisation for Economic Co-operation and Development) has stated that the SSC has become a core focus of the policies of their member countries government administration. The majority of developed countries and many developing countries have already adopted the SSC as a model for their service operations (OECD, 2010). It can be assumed that the SSCs in the public sector are part of the innovations in continuous growth brought by the NPM movement, as well as the customer orientation, e-government, control of results and professionalisation of management (Gonzalez et al., 2013). The autonomy to managers brought by NPM has fostered decentralised organisational models and the emergence of numerous redundancies in support functions such as finance, accounting, HR, and IT. The SSC was brought to public management as a way to correct this dysfunction and reduce operating costs (Janssen \& Joha, 2006; Tomkinson, 2007).

\subsubsection{Example of Benefits - Expansion of SSC in Local Governments in the UK and Losses in Other Countries}

Because of the financial crisis, the UK's public-sector spending faced greater scrutiny. The recession that followed the crisis saw the United Kingdom's government taking measures to cut progressively public spending using SSC as an organisational model for the public service. The intention behind the decision was to lower back office 
costs; with estimated savings of approximately $£ 20$ billion attributed to the implementation of the model (Turle, 2010). Although the UK's implementation of the model appears to be a success, other governments have been less successful. For example, the Government of Western Australia has accrued a billion dollars in losses. More pertinently, other governments have abandoned the SSC altogether and returned to the old, decentralised model (NCA, 2015).

SSCs in public management were set up with the external focus due to the New Public Management philosophy. However, there is evidence to suggest that there has been a trend toward an internal focus, which has resulted in an array of problems (see below). For this reason, this thesis seeks to understand whether the public SSC has an outside orientation (market orientation) or an inside orientation.

\subsubsection{Example of Services Provided by the SSC in Local Governments}

The public managed SSC provides services typical to its privately managed counterpart. For example, finance and accounting, HR, procurement and internal audits. Along with this, sector specific services are also provided; some of which are: welfare provision, building control and maintenance, waste collection, homeless accommodation, emergency planning and healthcare (Tomkinson, 2007). These are just a few examples of the extensive range of services covered by publically managed SSCs. A more in-depth look reveals a variety of services offered either directly to citizens or other agencies and government departments.

\subsubsection{Three Cases to Illustrate Problems with Shared Services Centres}

An analysis of three SSC projects (see Appendix A) that had partially or totally failed, exemplify the risk that governments and non-profit organisations face when 
embarking on these complex internal ventures, with inside orientation. The first two cases are from Governments (the United Kingdom and Australia) who experienced great challenges during the SSC implementation phase. The third case is an American University (Yale), which ran into difficulties during the running of the SSC. The main causes of failure in the former case were the inability of governments to balance between political and technical affairs as well as bureaucratic interference. In the second case, Yale, the project managers seem to have been insensitive to the customers of the SSC (NAO, 2008). The study revealed that a key fault, common to all three cases was a lack of orientation to their respective market. This situation led organisations to dissociate themselves from their customers and other stakeholders.

As we have seen in the discussions in the sections above, SSCs have expanded considerably in the public sector in several countries, bringing countless benefits and billions of dollars in avoided costs. However, we have also seen that there are several cases of failure, which have caused significant losses of public money. The understanding of the limitations of public SSC models and the extent to which these limitations are related to potential failures is a contribution that this study intends to offer to public managers. Preliminary analyses have led me to assume that SSC models copied from the industry by the public sector do not have all the features necessary for public SSC to function properly in the public context. The understanding of which characteristics of the SSC would need to be structured differently in the public sector, minimizing the risk of failure, is one of the expected products of this research. In section 2.6, I examined seven organisational elements (culture, leadership, resources, readiness for change, excellence, market orientation, performance) present in a service operation like an SSC. There is evidence that these elements can have a critical role in the success 
of an SSC (Bergeron, 2002; Robbins, 2011; Richter \& Brühl, 2016; NAO, 2008). Also in Section 2.6, these elements are structured as variables in a conceptual model so that the relationship between these variables is verified empirically.

\subsection{Conceptual Model: Modelling, Literature Review of Each Concept and the Proposed Model}

This section is dedicated to the conceptualisation, presentation, and discussion of the conceptual model developed during the literature review, whose main purpose is to

elucidate the research problem that aims to examine organisational factors that influence the performance of Shared Services Centres (SSCs). The conceptual model is the starting point of the quantitative part of the inquiry, which, in its whole, combines the qualitative and quantitative approaches, as explained in Chapter 1 and detailed in Chapter 3. The following is the first part of the discussion of the conceptual model, Building the Conceptual Model that conceptualises this type of model and clarifies which steps were fulfilled to reach the proposed model. The second part, Choosing Already Validated Constructs, focuses on the choice of constructs that would compose the model, detailing the applied selection criteria and exemplifying which constructs were considered or not. The third part, Literature Review of each Conceptual Model's Concept, brings the reviewed literature of each concept that makes up the conceptual model, contextualising existing theory and formal knowledge about the theme and its relevance to this study. The last part, The Proposed Conceptual Model, presents the conceptual model resulting from the modelling and discusses each of its components (constructs). It also shows how the constructs were tested and validated in previous studies, discussing their relationship with other constructs of the proposed model and the hypotheses I established that were later tested and validated in the field. 


\subsubsection{Building the Conceptual Model}

Here an introduction to the theme 'conceptual model' is provided, and discussed for what purpose and how the conceptual model proposed by this thesis was conceived.

What is a conceptual model - Conceptual model is a diagram, built on concepts and theory, that presents the main elements of a 'system' under study with relationship hypotheses between such elements (Jonker \& Pennink, 2008). The theory allows the identification of the variables (constructs) and the relationship between them so that hypotheses can be generated and tested empirically. Without the theory, it would not be possible, for example, to create an interpretation of reality up front, and build the proposed model. Theory instructs us on what to look at, how to look, and where to look. The conceptual model also helps in the visualisation of ideas and concepts and how their components are interrelated. The conceptual model intends to be an informative and revealing version of the reality under study. In the case of management, this is a simplified representation of complex organisational phenomena, which enables the mathematical formulation of such phenomena to be tested experimentally (Culyer, 2014). Thus, the mathematical model designed represents and allows to study the part of the real world of interest. This was the main reason for adopting such a quantitative approach in this thesis. And that is, because it is adequate to study the factors that influence SSC performance. Conceptual modelling has proven an important tool, since it allowed to establish the scope of the study and to understand the relationship between the factors analysed, and if in fact, each factor contributes to the performance of the SSCs.

Measurement scales - Every mathematical model needs evidence to feed its parameters. One of the ways to obtain such evidence is field surveys (e.g. 
questionnaires), using already existing measurement scales, carefully designed to measure each specific construct (latent variable). As the construct is a concept, it cannot be measured directly (Culyer, 2014; Easterby-Smith et al., 2008), and the measurement scales fulfil this role. It is important to verify that such scales had good performance when applied in the studies of origin. More information about scales of measurement can be found in Section 3.8. Questionnaire and Measurement Scales.

Three main purposes of a conceptual model - According to Jonker \& Pennink (2008), a conceptual model exists to fulfil three main roles: (1) The first role of a conceptual model is to associate research with the existing body of knowledge. The conceptual model shows how the researcher is approaching the researched phenomenon. The theories and concepts considered in the elaboration of the conceptual model show from which perspective the phenomenon is being approached. Furthermore, what factors were chosen to be analysed connecting the current research to other works. This makes it possible to justify the inquiry at a theoretical level. (2) The second role of a conceptual model is to help better structure the research problem since while building the model it is necessary to revisit the research problem several times. The model can be a good representation of the phenomenon under study and help to simplify it, because only the main factors end up remaining. This contributes to giving focus to what is essential for understanding the phenomenon. (3) The third role of a conceptual model is to enable the analysis of the phenomenon under the lens of systems theory. This allows, for example, that we analyse the components of the model in their relationships to verify how the elements interact dynamically in situations of cause and effect. 


\subsubsection{Choosing Already Validated Constructs}

This part of the document discusses the process applied for identification and selection of constructs, which should compose the conceptual model proposed by this thesis.

A conceptual model can be built with new constructs, developed from scratch from the theory, especially for a given research. In this case, the research project time may take some years between the proposal of the conceptual model, the validation of the measurement scales and the validation of the model hypotheses. However, the most common in quantitative research is, whenever applicable, to search for individual constructs already validated in previous studies, and with them to form a new conceptual model adequate for the scope and research problem of the study (Jonker \& Pennink, 2008; Hair, 2006) and use it. This last one was the approach adopted in this thesis, because it was established as a premise to the use of existing constructs, as a matter of time restriction to the development of the research project.

Next, I discuss the method adopted for conceptual modelling in this work. Since no established method of conceptual modelling was found during the literature review, I chose to develop my method, based on the recommendations of Jonker \& Pennink (2008) and Tappenden (2012). It is a two-stage method that leads the modeler from the identification of the factors relevant to the phenomenon studied, to the final modelling.

Stage 1 Identification of constructs potentially useful - The first stage in the conceptual modelling method used in this thesis was to identify potentially applicable constructs (organisational factors) that could express the analysed phenomenon, making it possible to model the reality of the research. For this thesis, the potentially applicable constructs are those that influence the performance of organisations 
(applicable to SSCs). In this way, the first step was to seek previous studies whose conceptual models or individual constructs influence the performance of organisations. To do this, I followed five steps: (a) I defined keywords relative to operations management, constructs, conceptual model, quality, and measurement scales; (b) I searched academic research databases accessible via the Discover mechanism of the University of Liverpool with access, for instance, to virtually all Business Management and Public Sector journals; (c) I selected articles through systematically reading the title, abstract and methodology section giving preference to articles whose objective was the development of measurement scales; (d) I expanded the search via Google Scholar; and after dozens of articles thoroughly examined, (e) I found 38 studies with constructs potentially applicable. It is important to note that I tried to start the search for already existing constructs quite openly, as explained in item (a) above so that I could find as many potential constructs as possible.

Table 2.1 below lists the 38 studies that I identified as potentially useful, and which brought more than 180 constructs as the source for my analysis and selection. The constructs in bold in the table are the most relevant for this thesis, and the articles with highlighted numbers show the articles whose constructs were selected at the end of Stage 1. Soon after the table, I comment on how I examined some of these works, to exemplify the construct analysis and filtering activity. 
Table 2.1 Potential sources of constructs

\begin{tabular}{|c|l|}
\hline$\#$ & Article name \\
\hline $\mathbf{0 1}$ & $\begin{array}{l}\text { Measuring critical success factors of TQM implementation } \\
\text { successfully - a systematic literature review }\end{array}$ \\
\hline 02 & $\begin{array}{l}\text { Market orientation and new-to-the-world products: exploring } \\
\text { the moderating effects of innovativeness, competitive strength }\end{array}$ \\
\hline 03 & $\begin{array}{l}\text { The effects of perceived market and learning orientation on } \\
\text { assessed organisational capabilities }\end{array}$ \\
\hline $\mathbf{0 4}$ & $\begin{array}{l}\text { Service orientation: the derivation of underlying constructs and } \\
\text { measures }\end{array}$ \\
\hline 05 & $\begin{array}{l}\text { Market orientation: A meta-analytic review of its antecedents } \\
\text { and impact on performance }\end{array}$ \\
\hline $\mathbf{0 6}$ & $\begin{array}{l}\text { Implementing Marketing Strategies: Developing and Testing a } \\
\text { Managerial Theory }\end{array}$ \\
\hline 07 & $\begin{array}{l}\text { Market orientation, marketing capabilities, and firm } \\
\text { performance }\end{array}$ \\
\hline 08 & $\begin{array}{l}\text { Market Orientation and Performance of Export Ventures: The } \\
\text { Process Through Marketing Capabilities and Competitive } \\
\text { Advantage }\end{array}$ \\
\hline $\mathbf{0 9}$ & $\begin{array}{l}\text { An empirical assessment of the EFQM Excellence Model: } \\
\text { Evaluation as a TQM framework relative to the MBNQA Model }\end{array}$ \\
\hline 10 & $\begin{array}{l}\text { Market orientation, service quality and business profitability: a } \\
\text { conceptual model and empirical evidence }\end{array}$ \\
\hline $\mathbf{1 1}$ & $\begin{array}{l}\text { Market orientation of service networks: direct and indirect } \\
\text { effects on sustained competitive advantage }\end{array}$ \\
\hline 12 & $\begin{array}{l}\text { A meta-analysis of the relationship between market orientation } \\
\text { and business performance: Evidence from five continents }\end{array}$ \\
\hline 13 & $\begin{array}{l}\text { Transformational leadership and market orientation: } \\
\text { Implications for the implementation of competitive strategies } \\
\text { and business unit performance }\end{array}$ \\
\hline 14 & $\begin{array}{l}\text { Achieving service excellence-measuring the impact of } \\
\text { leadership and senior management commitment }\end{array}$ \\
\hline $\mathbf{1 5}$ & $\begin{array}{l}\text { Measurement of perceived organisational readiness for change } \\
\text { in the public sector }\end{array}$ \\
\hline 16 & $\begin{array}{l}\text { Organisational readiness for change, individual fear of change, } \\
\text { and sales manager performance }\end{array}$ \\
\hline & (D) \\
\hline
\end{tabular}

Authors

(Hietschold et al.,

2014)

(Augusto \&

Coelho, 2009)

(Celuch et al,

2002)

(Oliveira \& Roth,

2012)

(Kirca et al., 2005)

(Noble \& Mokwa,

1999)

(Morgan et al.,

2009)

(Murray et al.,

2011)

(Bou-Llusar et al.,

2009)

(Baker \& Sinkula,

1999)

(Evanschitzky,

2007)

(Cano et al., 2004)

(Menguc et al.,

2007)

(Prabhu \& Robson,

2000)

(Cinite et al.,

2009)

(Weeks et al.,
Considered constructs (bold $=$ more relevant for this thesis)

Quality culture; Benchmarking; Top management commitment and leadership;

Process management; Customer focus and satisfaction; Supplier partnership; Strategic quality planning; Social and environmental responsibility.

Customer orientation; Competitor orientation; Interfunctional coordination;

Technological turbulence; Competitive intensity.

Marketing capabilities; Product/service capabilities; Global capabilities; Technical capabilities; Information systems capabilities; Upper management capabilities. Service culture; Measurement system; Service standards; Value orientation; Leadership; Market acuity; Relationship management; New service development.

Market orientation; Innovativeness; Customer loyalty; Quality; Organisational performance.

Organisational Buy-in; Championing; Senior Management Support; Organisational Commitment; Strategy Commitment; Role Commitment; Role Performance.

Market orientation; Marketing capabilities interaction; Marketing capabilities; Firm performance.

Market Orientation; Internal factors; External factors; Marketing capabilities; Competitive advantages; Performance.

Leadership; Society results; Policy and strategy; People; Partnerships and resources; Processes; Customer results; People results; Key performance results.

Market orientation; Service quality; Business Performance.

Resources (financial, physical, human, skills and competencies); Intangible Resources; Customer orientation; Inter-partner coordination; Market attractiveness.

Market orientation; Business performance; Business objective; Industry.

Transformational leadership; Market orientation; Innovation differentiation;

Marketing differentiation; Low-Cost strategy; Effectiveness; Efficiency.

Operational performance; Senior management commitment; Employee-related practice

Readiness for change; Unreadiness for change.

Organisational readiness to change; Individual fear of change; Job performance. 


\begin{tabular}{|c|c|c|c|}
\hline \# & Article name & Authors & Considered constructs (bold = more relevant for this thesis) \\
\hline 17 & $\begin{array}{l}\text { In search of excellence revisited: an empirical evaluation of } \\
\text { Peters and Waterman's attributes of excellence }\end{array}$ & $\begin{array}{l}\text { (Sharma et al., } \\
\text { 1990) }\end{array}$ & Excellence. \\
\hline 18 & $\begin{array}{l}\text { Relationship orientation or service quality?: What is the trigger } \\
\text { of performance in financial and insurance services? }\end{array}$ & (Camarero, 2007) & Service quality; Market orientation; Relationship Orientation. \\
\hline 19 & $\begin{array}{l}\text { The desired level of market orientation and business unit } \\
\text { performance }\end{array}$ & $\begin{array}{l}\text { (Song \& Parry, } \\
\text { 2009) }\end{array}$ & $\begin{array}{l}\text { Market environment; Innovation strategy; The desired level of market orientation } \\
\text { (DMO); GAP; Business unit performance. }\end{array}$ \\
\hline 20 & $\begin{array}{l}\text { Corporate culture, customer orientation, and innovativeness in } \\
\text { Japanese firms }\end{array}$ & $\begin{array}{l}\text { (Deshpandé et al., } \\
\text { 1993) }\end{array}$ & Culture; Customer orientation; Innovativeness. \\
\hline 21 & $\begin{array}{l}\text { Building organisational capabilities for managing economic } \\
\text { crisis: The role of market orientation and strategic flexibility }\end{array}$ & $\begin{array}{l}\text { (Grewal \& } \\
\text { Tansuhaj, 2001) }\end{array}$ & Environment; Market orientation; Strategic flexibility; Performance after crisis. \\
\hline 22 & $\begin{array}{l}\text { Do frontline mechanisms matter? Impact of quality and } \\
\text { productivity orientations on unit revenue, efficiency, and } \\
\text { customer satisfaction }\end{array}$ & $\begin{array}{l}\text { (Marinova et al., } \\
\text { 2008) }\end{array}$ & $\begin{array}{l}\text { Quality orientation; Productivity orientation; Unit autonomy; Unit cohesion; } \\
\text { Performance feedback; Unit revenue; Unit efficiency; Customer satisfaction. }\end{array}$ \\
\hline 23 & $\begin{array}{l}\text { Creating a firm-level dynamic capability through capitalising on } \\
\text { market orientation and innovativeness }\end{array}$ & $\begin{array}{l}\text { (Menguc \& Auh, } \\
2006 \text { ) }\end{array}$ & $\begin{array}{l}\text { Customer orientation; Competitor orientation; Interfunctional coordination; } \\
\text { Innovativeness; Market turbulence; Competitive intensity; Technological turbulence. }\end{array}$ \\
\hline 24 & $\begin{array}{l}\text { Understanding market orientation: A prospectively designed } \\
\text { meta-analysis of three market orientation scales }\end{array}$ & $\begin{array}{l}\text { (Deshpande \& } \\
\text { Farley, 1996) }\end{array}$ & Market Orientation. \\
\hline 25 & $\begin{array}{l}\text { In Search of Excellence: Lessons from America's Best Run } \\
\text { Companies }\end{array}$ & $\begin{array}{l}\text { (Peters et al., } \\
\text { 1982) }\end{array}$ & Excellence in business. \\
\hline 26 & $\begin{array}{l}\text { Customer-based corporate reputation of a service firm: scale } \\
\text { development and validation }\end{array}$ & $\begin{array}{l}\text { (Walsh \& Beatty, } \\
\text { 2007) }\end{array}$ & $\begin{array}{l}\text { Customer orientation; Good employer; Reliable and financially strong company; } \\
\text { Product and service quality; Social and environmental responsibility. }\end{array}$ \\
\hline 27 & $\begin{array}{l}\text { Using performance measures in public organisations: } \\
\text { challenges of Italian public administrations }\end{array}$ & $\begin{array}{l}\text { (Sole \& Schiuma, } \\
2010)\end{array}$ & Goal orientation; Resources for performance measurement; Rewards; Attitudes. \\
\hline 28 & $\begin{array}{l}\text { Commitment to Organisations and Occupations: Extension and } \\
\text { Test of a Three-Component Conceptualisation }\end{array}$ & $\begin{array}{l}\text { (Meyer et al., } \\
1993 \text { ) }\end{array}$ & $\begin{array}{l}\text { Affective occupational; Continuance occupational; Normative occupational; Affective } \\
\text { organisational; Continuance organisational; Normative Organisational. }\end{array}$ \\
\hline 29 & The Measurement of Organisational Commitment & $\begin{array}{l}\text { (Mowday et al., } \\
\text { 1979) }\end{array}$ & Organisational commitment. \\
\hline 30 & $\begin{array}{l}\text { Enterprise Resource Planning (ERP) Competence Constructs: } \\
\text { Two-Stage Multi-Item Scale Development and Validation }\end{array}$ & $\begin{array}{l}\text { (Stratman \& Roth, } \\
\text { 2002) }\end{array}$ & $\begin{array}{l}\text { Executive commitment; Project management; IT skills; Business process skills; ERP } \\
\text { training; Learning; Change readiness; Improved business performance. }\end{array}$ \\
\hline 31 & $\begin{array}{l}\text { The interplay of different types of governance in horizontal } \\
\text { cooperations: A view on logistics service providers }\end{array}$ & $\begin{array}{l}\text { (Raue \& Wieland, } \\
\text { 2015) }\end{array}$ & Formalisation; Relational capital; Performance; Learning. \\
\hline 32 & Development and validation of TQM implementation constructs & (Ahire et al, 1996) & $\begin{array}{l}\text { Top management commitment; Customer focus; Supplier quality management; } \\
\text { Design quality management; Benchmarking; Statistic process control usage. }\end{array}$ \\
\hline 33 & $\begin{array}{l}\text { Organisational Culture and New Service Development Performance: } \\
\text { Insights from Knowledge Intensive Business Service }\end{array}$ & (Liu, 2009) & Performance. \\
\hline 34 & Linking routines to operations capabilities: A new perspective & (Peng et al., 2011) & $\begin{array}{l}\text { Continuous improvement; Process management; Leadership involvement in quality; } \\
\text { Search for new technologies; Cross-functional product design. }\end{array}$ \\
\hline
\end{tabular}


Chapter 2

\begin{tabular}{|c|l|l|l|}
\hline$\#$ & Article name & Authors & Considered constructs (bold = more relevant for this thesis) \\
\hline 35 & $\begin{array}{l}\text { New service development competence in retail banking: } \\
\text { Construct development and measurement validation }\end{array}$ & $\begin{array}{l}\text { (Menor \& Roth, } \\
\text { 2007) }\end{array}$ & $\begin{array}{l}\text { New service development process focus; Market acuity; New service development } \\
\text { strategy; New service development culture; Information technology experience. }\end{array}$ \\
\hline 36 & $\begin{array}{l}\text { Accounting for external turbulence of logistics organisations } \\
\text { via performance measurement systems }\end{array}$ & $\begin{array}{l}\text { (Bühler } \text { et al., } \\
2016)\end{array}$ & $\begin{array}{l}\text { Attention focusing; Score keeping; PMS design for turbulence; Organisational } \\
\text { resilience; Distribution service performance; Customer orientation. }\end{array}$ \\
\hline 37 & $\begin{array}{l}\text { A framework for quality management research and an } \\
\text { associated measurement instrument }\end{array}$ & (Flynn et al., 1994) & $\begin{array}{l}\text { Top management support; Quality information; Process management; Product design; } \\
\text { Workforce management; Supplier involvement; Customer involvement. }\end{array}$ \\
\hline 38 & A Refinement and Validation of the MARKOR Scale & $\begin{array}{l}\text { (Matsuno } \text { et al., } \\
2000)\end{array}$ & Intelligence generation; Intelligence dissemination; Responsiveness; Performance. \\
\hline
\end{tabular}


The following, I use three examples to demonstrate how I examined the selected articles. The first example illustrates an article selection that presented a relevant construct for the conceptual model proposed by this thesis. The second and third examples show article analysis whose constructs were discarded, since they could not be used as factors that influence the performance of an organisation, directly or indirectly.

Example 1 (article \#04 of Table 2.1 above) Identification of relevant constructs - The initial selection of the article occurred as described in steps (b) and (c) above. In possession of the article, I proceeded with an initial reading to verify the methodological consistency of the work examining its validity and reliability (Easterby-Smith et al., 2008), and which constructs could actually be useful. Oliveira \& Roth (2012) in this study elaborated Service Orientation constructs; that is, the propensity of the organisation to deliver excellence in service. Examples of such constructs are Service Culture, Measurement System, Service Standards, Value Orientation, Leadership, Market Acuity, Relationship Management and New Service Development, and they have developed their respective measurement scales empirically through research involving 181 senior managers. It is interesting to note that the article makes clear the relationship of these constructs with excellence in service. In analysing carefully the constructs proposed by the authors, I verified that four could be useful: Service culture (the measurement scales could be applied in a broader construct 'Organisational Culture'), with standardised path loading 0.86 (it should be greater than 0.60); Measurement system (measurement scales could be applied in a broader construct 'Performance'), with standardised path loading 0.82; Service standards, with standardised path loading 0.84 and Value orientation, with standardised path loading 0.82 (both could be applied in a broader construct 'Excellence'). This initial assessment 
came through insights from the article itself and literature review, and showed that these constructs have the potential to verify the contribution of factors to the performance of an organisation, and this property was confirmed in Stage 2, discussed below. Although this evaluation is not conclusive at this point, it acts as an effective filter in the pre-selection of potential constructs.

Example 2 (article \#03 of Table 2.1 above) Discarded constructs - Initially, what caught my attention in this article was the association of the construct Upper Management Capabilities with the construct Market Orientation. My initial expectation was to understand how the first construct was developed, if, for example, encompassed or not leadership and which measurement scales have been proposed by the researchers Celuch et al. (2002). While analysing the article in this stage, I verified that the first construct, Upper Management Capabilities, includes Leadership, Strategy, and Planning, but to my surprise, the article does not bring the measurement scales, making its use impossible in my study.

Example 3 (article \#16 of Table 2.1 above) Discarded constructs - This study highlights three constructs (Organisational Readiness to Change, Individual Fear of Change, Job Performance), with measurement scales validated by 340 sales managers. It was pre-selected so that I could look more closely at the construct Organisational Readiness to Change. Two aspects of the analysis led me to discard it. First, the measurement scale of the construct of my interest was not very comprehensive (low coverage) when compared to other studies, such as, for example, Cinite et al. (2009). Second, the performance studied is that of the individual and not the organisation as would be of my interest. However, although the article has been dismissed, it brought an important contribution highlighting and justifying the relevance of organisational culture to the overall 
performance of an organisation. Given I was in search of constructs related to organisational performance, it has helped.

A conceptual model should have as few constructs as possible explaining the phenomenon, to comply with the principle of simplification (Jonker \& Pennink, 2008; Easterby-Smith et al., 2008). When one thinks of constructs associated with Organisational performance, one can expect to work with concepts such as economies of scale, specialisation, effectiveness, efficiency, and relationship. However, in the searches carried out constructs related to these themes that could apply to this thesis were not found; on the other hand, it was identified the construct Resources that allowed to study the influence of the organisational resources in the performance of SSCs. Other concepts/constructs, which were located and could, eventually, be adopted, were purposefully left out of scope. This is the case, for example, of constructs such as Customer Orientation, dropped because the concept was attended by the construct Market Orientation, and Executive Commitment, whose concept has been met by the construct Leadership. Another example of a construct thrown out by simplification of scope is New Service Development, which could influence the performance of an organisation while defining its service portfolio. For the study conducted in this thesis, it was evaluated as having less impact in comparison to the other selected constructs.

In Stage 1, to each analysed article there were countless queries to the reviewed literature, revisits to the research problem and complementary analyses, which is common during the design of a conceptual model (Jonker \& Pennink, 2008). From this interaction and informed analysis, it was possible to identify the seven concepts/constructs (culture, leadership, resources, readiness for change, excellence, market orientation and performance) considered relevant to be treated in Stage 2 as to 
the causality and relationship with other constructs, and the formulation of hypotheses, namely, the elaboration of the proposed conceptual model, discussed in the following.

Sage 2 Selection of final constructs - The second stage aims to ratify the relevant factors identified in Stage 1. The Stage 2 begins when Stage 1 already has sufficient knowledge produced and not at the end of Stage 1. One of the reasons for this is that in reviewing the articles in Stage 1, it is possible to identify several relationships between constructs confirmed in those studies, and this helps in choosing more relevant constructs (in my case, the factors that influence the performance of an SSC) and beginning of the modelling.

To ratify the constructs recommended by Stage 1, the key question in Stage 2 is 'What is relevant?' In this case, relevant is a small number of factors that have a significant influence on the performance of SSCs. Relevance may have been verified in empirical studies researched or be established by the researcher of the new study (Jonker \& Pennink, 2008; Easterby-Smith et al., 2008).

However, to ratify the relevant factors, first of all, I followed two suggestions of Jonker \& Pennink (2008). First, I began to sketch the conceptual model, and second, I put more on the right in the sketch the concept that will be explained by the model; in my case, Performance. In this way, the concepts used to explain Performance were more on the left of the diagram. Another insight for modelling was that during the literature review, it became clear that the New Public Management movement (which gave rise to SSCs in the public sector) emphasises the orientation of the public service to its clients/citizens. This, translated in terms of existing conceptual models and constructs, means Market Orientation (Deshpande \& Farley, 1996). Thus, I already had two 
constructs ratified as relevant, early in the modelling: Performance and Market Orientation.

After the filtration established in Stage 1, of the 38 studies with potentially applicable constructs, remained 11 studies, shown with article numbers highlighted in Table 2.1 above, which propose the constructs considered most relevant to this thesis (culture, leadership, resources, readiness for change, excellence, market orientation, and performance). In Stage 2, the modelling followed four steps (1) outlining the initial diagram of the conceptual model, starting with the Performance and Market Orientation constructs, as discussed above; (2) revisits to each of the articles from which the constructs considered to be relevant have been extracted, and carry out additional researches in the literature to better understand the scope of each construct and its proven relationship with other constructs; (3) complementation of the conceptual model diagram with each relevant construct ratified, including visual identification of the relations of causality between the constructs, based on the articles of origin and complementary literature; (4) conclusion of the conceptual model diagram and establishment of the hypotheses to be tested empirically.

To illustrate step (2) I cite an example of complementary research for the Excellence, Market Orientation and Performance constructs: the study of Caruana et al. (1999) confirmed the relationship between these constructs and helped me in the initial modelling. This procedure is discussed in more depth in Section 2.6.4 The Proposed Conceptual Model.

The following is a review of the theory regarding each dimension (concept) of the conceptual model. 


\subsubsection{Literature Review of each Conceptual Model's Concept}

A conceptual model can direct a research by providing a graphic representation of the variables (theoretical constructs) to be studied. The conceptual models express a systematic view of a phenomenon, through the specification of the relationship of a set of variables, and the resulting hypotheses (Creswell, 1994). To build a conceptual model is necessary to perform a thorough literature review to find the variables of interest for the model, and the measurement scales empirically tested. This section introduces the concepts and theories intended for design, development, and review of the conceptual model developed for this research (see Section 2.6.4 The Proposed Conceptual Model). They encompass the following areas of study: culture, leadership, resources, readiness for change, excellence, market orientation, and performance. Later, in Section 2.6.4, these elements are linked as relevant factors for an SSC service operation. It is interesting to note that the public sector SSCs, especially those with a New Public Management-related origin, should be market-oriented and each of the seven elements mentioned above should contribute to this.

\subsubsection{Culture}

Culture can be defined as "the collective programming of the mind which distinguishes the members of one human group from another" (Hofstede, 1984, p.21). In other words, culture pertains to the thought habits of a people and their subsequent actions (Liu, 2009). More than that, culture plays a key role in the success of all organisation's dimensions (Cameron \& Quinn, 2005). Organisational culture should not be stagnant; rather, success in a dynamic business environment requires it to adapt to changing circumstances to survive, for instance, through its re-enactment (Schein, 1999; Anderson \& Anderson, 2010). This is particularly crucial in a market-oriented 
organisation, which often operates in a highly turbulent environment, and it is necessary a deeper analysis of its basic values, artefacts, behaviours and standards (Homburg \& Pflesser, 2000). Several studies (e.g. Tomaskova, 2015) have identified culture as a major barrier to the adoption of Market Orientation (MO). A closed culture, whether strong or weak, it tends to direct the organisation to have internal focus; and open culture, too weak or strong, tends to direct the organisation to have an external focus. Therefore, the more open to its business environment is the organisational culture, the more it will favour the adoption of MO (Hayden, 1993; Deshpandé \& Farley, 2004).

Before embarking on a journey of cultural transformation aiming at the MO, the organisation needs to define how market-oriented it is, and as it should be to maximise the performance (Song \& Parry, 2009). However, why is it important to change? Why is change a need? Usually, the answer is related to the mission of the organisation, its external environment, and its current performance. A diagnosis of organisational culture can help the organisation to understand better their cultural characteristics most relevant. Also, it can help to establish a plan to enhance the cultural elements lagged (Cameron \& Quinn, 2005). Studying MO, Gebhardt, Carpenter and Sherry Jr (2006) identified that cultural transformation was the common aspect decisive for companies to become market-oriented successfully. They concluded that cultural artefacts enhanced gave the necessary support, not only for the organisations surveyed become MO, but also to rebalance the intra-organisational power properly. Moreover, the reviewed researches showed that, usually, cultural change is triggered by external factors, also in non-profit organisations (Gainer \& Padanyi, 2005).

To shape an organisation through a MO cultural lens, it is important to consider how an organisational culture can be more effective in creating the behaviours needed 
to generate higher value for customers and providing thus a superior performance for a sustainable business (Narver \& Slater, 1990). According to these authors, there are three behavioural components leading to the MO: (a) the customer orientation; (b) the functional coordination; and (c) the orientation to the competition. It is worth pointing out that, although in the public sector there is usually no market competition, and in the case of SSC, the characteristics and quality of the service need to be compared with what is on offer by other organisations. These include private sector companies (that have direct dealings with the public), who can be used as a quality benchmark to compare with public services. This is necessary because an SSC operation runs like a business unit; thus, it needs to be oriented to its corresponding market. A market-oriented SSC must have a culture that inspires high-quality customer service in the short, medium and long-term; this allows the organisation to provide appropriate responses to the expectations of the customers (Liu, 2009; Dessein \& Santos, 2006). Finally, the culture must promote the customer/citizen to the heart of the mission of the service operation. There are also evidences from SSC studies that culture is an important issue to be addressed (e.g. Janssen \& Joha, 2006; Schwarz \& Chakraborty, 2012; Persson \& Göransson, 2012).

\subsubsection{Leadership}

Proper leadership is a factor that determines the success or failure of an operation of services such as the SSC. The attitude of the first level management on market orientation (MO) is the major determining factor in whether this business model (SSC) is adopted by an organisation (Tomaskova, 2015). According to Kotter (2001), leadership rests on successfully governing change; thus, the primary role of a leader is to exercise influence over the behaviour of a group to ensure that their actions are 
conducive to achieving the necessary goals (Robbins, 2011). An effective leader is, for instance, proactive and decisive. She or he guides employees towards achieving the mission of the organisation and motivates them to provide quality customer service. Furthermore, an effective leader not only advocates the quality and excellence of services but also acts to set an example to employees, discussing important operational issues with the employees and engaging with customers. Senior management should be prepared to influence and to conduct the organisational changes (explored below) necessary for the evolutionary adaptation of a service operation (Cinite et al., 2009).

It is not possible to deploy MO without a leadership duly prepared to conduct the necessary transformation of the organisation (Menguc et al., 2007; Prabhu \& Robson, 2000). Empirical studies have demonstrated that there is a positive correlation between an effective leadership and the market success of distinguished, high-performance companies (Prabhu \& Robson, 2000). Leadership plays a crucial role in the cultural transformation that will direct the organisation towards market-orientation. There are at least four critical leadership actions for an organisation to become market-oriented: (a) establishing a pact to direct the organisation to MO; (b) establishing a vision of MO and the steps for its deployment; (c) communicating the vision; and (d) empowering the employees to perform the necessary actions so that the organisation becomes MO (Narver et al., 1998).

I analysed the three paradigms and models of leadership from Kanji \& Sá (2001): (a) transcendent leadership, which cultivate the transcendent motivation of their supporters; (b) transactional leadership, which motivate followers mainly through contingent-reward-based exchanges; and (c) leadership transformation, which try to inspire followers to accomplish great things, acting as strong role models). Among them, 
just transformational leadership seems to be the most appropriate conceptual approach to driving the change required in adopting MO. This approach is the one most suited to perform change management and organisational transformation. Unlike transactional leadership, for example, transformational leaders work to establish a vision and encourage their followers to achieve it. This inspiration is based more on truth and commitment than on contractual agreements, as occurs in transactional leadership.

\subsubsection{Resources}

Since the public sector organisations exist not to achieve profit, but to meet the needs of particular groups of customers/citizens, the strategic approaches in the public sector are focused on how to maximise the organisational performance, and not on how to increase profitability (Pablo et al., 2007). Therefore, an "outside-in approach (typically used by companies, not governments), as the Resource-Based View (RBV) is quite suitable for the study of how to monitor or evaluate the performance of an SSC in public management because the RBV studies the success of companies from their internal resources (Evanschitzky, 2007; Wernerfelt, 1984). Born originally to explain the competitive advantage of businesses in the industry, the RBV can help explain the performance of a public sector service operation showing to what extent its resource groups contribute to a better performance (Peteraf, 1993; Wernerfelt, 1984). The RBV believes that the source of competitive advantage is the organisation's resources and that there is a unique configuration of these resources and capabilities in each company (heterogeneity), distinctive competencies difficult to be copied by competitors (Barney, 1991; Mahoney \& Pandian, 1992).

In the perspective of the RBV framework, there is an important distinction between the enterprises with profitable ends in comparison with the government 
organisations as for the treatment given to the intellectual capital, the knowledge resource (Syed-Ikhsan \& Rowland, 2004; Landry et al., 2006). Many private companies have serious limitations in capturing, structuring and disseminating knowledge which their collaborators hold. Employees perform procedures are not always well documented, limiting the identification and transfer of knowledge, as well as its use as a business resource. In government organisations, the procedures are all documented (sometimes to excess) to ensure that laws are abided by (Denhardt \& Denhardt, 2000). However, because of their hierarchical and bureaucratic structure, government organisations have difficulty disseminating the knowledge (Syed-Ikhsan \& Rowland, 2004). That being said, it is important to consider that the public SSCs also experience similar problems with its use of resource knowledge (Syed-Ikhsan \& Rowland, 2004).

The RBV have been criticised for a limited view of resources, especially for assuming that a firm has sufficient resources (Janssen \& Joha, 2007). Therefore, any research that addresses RBV must examine the dynamic capabilities, which are intended to bridge this RBV gap. The concept of dynamic capabilities aims to cover this gap addressing aspects not encompassed by RBV such as specific skills, processes, procedures and organisational structures. The dynamic capabilities can be defined as the company's ability to develop, join in and reconfigure external and internal skills to adapt to fast-changing environments (Teece \& Pisano, 1994; Teece, 2007). Other researchers define the dynamic capabilities, roughly speaking, as a specific set of business processes (Eisenhardt \& Martin, 2000). However, in general, all of them recognise that the dynamic capabilities allow the organisation to adjust their resources adapting to the demands of the environment in which it is inserted (Janssen \& Joha, 2007). 
From the reviewed literature, five groups of resources were considered in preparing the construct used in this thesis: (a) financial resources - relating to needed investments in operation; (b) physical resources - relating to premises and equipment; (c) human resources - which include individual skills; (d) organisational resources which include organisational skills; and (e) specific service-related resources such as the posture and practices in the provision of services (Evanschitzky, 2007). Although there is no consensus among authors on what 'resources' are, the RBV offers theoretical tools for an organisation's internal vision, and it is possible to reconcile the RBV with MO concerning the organisation of resources to make them more customer-oriented. For example, the MO may contribute with its Outside-in vision, complementing the predominantly Inside-out vision of RBV (Evanschitzky, 2007).

\subsubsection{Readiness for Change}

As discussed above, the commitment of senior management is critical to the success of an SSC. Similarly, the willingness of an organisation to adapt to the necessary changes is also an essential deciding factor (Kotter, 2001; Schulman et al., 1999; Todnem By, 2005). The readiness for change is part of the change management process, i.e. the process of continuously renewing the direction of the organisation, its structure, and resources to meet the ever-changing needs of internal and external customers (Moran \& Brightman, 2000). Furthermore, the readiness for change within the organisation is dependent on the perception (by members of the organisation) that change is necessary as well as its ability to effect change (Jansen, 2000). If an organisation cannot administer management of change, the transformation project is likely to fail; in its attempts to become market-oriented (Hayden, 1993). Although the technical aspect of the transformation project is important, the most critical factor to success is the people; they 
will be the most impacted by the project. The changes affect the emotions of the collaborators because of the stressful nature of the project. Therefore, a climate of confidence, transparency, and respect must be established between participants. Every change causes discomfort and insecurity in the people involved, and care must be taken. Thus, productivity and camaraderie across the organisation are not adversely affected, as a high-stress environment will have a negative effect on the success of the project (Weeks et al., 2004; Kotter, 2001; Jansen, 2000).

Studies indicate that around $50 \%$ to $66 \%$ of transformation projects fail to achieve their goals and attributes due to resistance to change, this is known as the "veiled cause" (Waddell \& Sohal, 1998). The literature on resistance to change has identified several possible causes that can be categorised into three main groups. A) Communication, such as lack of understanding about what is happening; lack of knowledge about the reasons behind the change; ignorance of the impacts that will be brought about by the change, etc. B) Political reasons, such as reduction of power; opposing interests; interference; choice of suppliers, etc. (C) Technical reasons, such as disagreement regarding the adopted solution; ignorance/fear of new technology; financial impact; technological impact; impact on competitiveness, etc. These causes indicate the extent of the resistance to change; also, there are other minor causes (Anderson \& Anderson, 2010; Weeks et al., 2004). Although resistance to change is usually associated with negative attitudes of staff, such as delays in the project, higher costs and changes to the original scope, it can have positive consequences such as encouraging staff to get better engaged and informed about the transformation, this ultimately means that they can be used as agents of change (Waddell \& Sohal, 1998; Anderson \& Anderson, 2010). 
The readiness for change can be approached at an organisational level which may require the alignment of processes and methodologies with business goals, and at an individual level, requires the appropriate skills to be developed through training and alignment of staff expectations. Studies suggest that organisations with a culture of continuous learning favour the individual readiness for organisational change (Choi \& Ruona, 2010). It is important to note that the readiness for change works in the organisational cultural framework and Kotter (1995) reminds us that the preparation of the organisation to be able to change should be reflected and absorbed by the culture of the Organisation in question. A construct of readiness for change developed and tested for the public sector was used in this research (Cinite et al., 2009).

\subsubsection{Excellence}

Although there are many studies addressing business excellence, there are few clear definitions of the subject. The following are discussed definitions of service excellence and business excellence. Studies on excellence in service state that this concept is related to providing excellent service quality exceeding the customer's initial expectations, generating satisfaction and customer loyalty (Gouthier et al., 2012). The definition of excellence in services is also addressed by Johnston \& Clark (2001) which argues that, normally, excellence in services is defined as "exceed expectations," but that this definition is insufficient for operationalisation. In his research, he found that service excellence is related more to 'be easy to do business with' than to exceed the expectations. He concluded that service excellence depends on four characteristics: (a) delivering what was promised; (b) providing customers with a personal touch; (c) going the extra mile; and (d) excellent complaint, problem and query handling (Johnston, 2004). 
As for business excellence, some researchers link the concept to excellence in quality together with excellence in other aspects of management, for example, finance, logistics, and human resources. They also argue that business excellence is a way of achieving high-quality service and thus, customer satisfaction (Klefsjö et al., 2008). Other researchers associate business excellence with the principles and management models of the European Foundation for Quality Management (EFQM) and the International Organisation for Standardisation (ISO) (Dale et al., 2000). According to the Merriam-Webster dictionary, excellence means "the quality of being excellent"; "an excellent or valuable quality" (Merriam-Webster, 2015a). That is, it is something that exceeds the usual standard, something that is better than the alternative (for example, in services).

Excellence can be only achieved if the use and optimisation of key resources are aligned with all areas of the company and stakeholder interests are highly considered. The pursuit of excellence in services is an ongoing process (Caruana et al., 1999; EFQM, 2015; Bou-Llusar et al., 2009). It necessitates the alignment of all operational processes towards the goal of achieving high standards in customer satisfaction (Marques, 2006). The analysis of organisational excellence needs to go beyond "pro-excellence posture" and practices. The patterns and levels of service quality, benchmarking mechanisms for continuous improvement, and its orientation to deliver value to customers must be considered (Sharma et al., 1990; Camarero, 2007; Hietschold et al., 2014; Noble \& Mokwa, 1999). Excellence, market orientation, and service quality are interrelated and result in a significant contribution to the business performance (Caruana et al., 1999; Chang \& Chen, 1998). 


\subsubsection{Market Orientation}

Briefly, Market Orientation (MO) is the pursuit of the strategic alignment of an organisation with the objective of making it more proactive and oriented to its external environment. There are two main conceptual approaches to MO. In the first, MO refers to the generation and propagation of market intelligence throughout all departments and the ability to respond to changing needs (Kohli \& Jaworski, 1990). In the second, MO is the organisational culture that creates the most efficient behaviour necessary for the generation of superior value to the customer and thus a better business performance (Narver \& Slater, 1990). According to the MO, to deliver quality services and products a company needs to monitor, understand, and respond to the needs of its clients in a continuously evolving environment (Jaworski \& Kohli, 1993). This requires the development of a strong customer orientation along with the capacity to satisfy their expectation (Bearden \& Netemeyer, 1999; Deshpande \& Farley, 1998). The company's ethos should reflect their objectives by including values such as a commitment to meeting the client's needs (Day, 1994). In addition, a common view of the market needs to be shared by the organisation's managers (Gebhardt et al., 2006). Researchers have highlighted a need for further research be carried out on MO in different scopes to ratify the MO/performance relationship (Narver \& Slater, 1990; Kohli \& Jaworski, 1990).

It is also important to understand that to have a more complete and useful market orientation, it is not enough to address the needs expressed by the customer (responsive market orientation), but it is necessary also to identify and give an answer to the latent customers' needs (proactive market orientation). Various researchers have demonstrated that proactive MO contributes directly to the success of new products launched (Narver et al., 2004). 
Despite MO applies to all market environments and all type of organisation can embrace it and adjust the degree of MO according to their characteristics and reality (Narver \& Slater, 1990), there are still a few market-oriented government organisations, but the trend is that there will be a considerable expansion of it. Usually, it is the existence of competition that leads an organisation to become market oriented. However, the pressure of customers/ citizens for better services and better use of public money can be a sufficient stimulus to this (Hayden, 1993). The lack of competition in the public service could cause their service operations not to be the best possible and MO can help identify opportunities for improvement. At this moment when the public sector organisations are seeking to become market-oriented, there is little research that can help them in this activity (Cervera et al., 2001). To improve the performance, MO should lead the company to act geared to competition, to customers and with inter-functional coordination (Walker et al., 2011). While the application of MO is a more common approach to private companies, public sector organisations can obviously benefit from market intelligence, and their dissemination to all its departments, as they can respond appropriately with the required form, content and agility (Cervera et al., 2001). When analysing the application of MO to public management, clearly there is a latent risk of putting the focus solely on customers/citizens, for the attention to the interests of the various stakeholder groups can determine the success of an organisation (Hult, 2011). In public management, two other important stakeholder groups, in addition to customers and citizens, should be considered (a) public servants; and (b) public managers (Walker et al., 2011). A better understanding of the expectations and needs of the customers/citizens may make a public organisation a more efficient service provider (Jaworski \& Kohli, 1993); also the MO can bring social and psychological benefits to the employees, creating a more pleasant working environment, employee team spirit, and 
generating a better level of employee commitment (Jaworski \& Kohli, 1993). Also, according to Harris \& Ogbonna (2001), managers need to be fully engaged with the MO for the functional coordination to work properly.

Even though the MO was seen in the 1990s as a competitive advantage, today it is so widespread in companies that have become more a necessity, an essential item to compete, than a competitive advantage. Only companies that have adopted a MO approach earlier might take advantage of it as an advantage to compete in their markets (Kumar et al., 2011). However, this does not limit its relevance. Dozens of studies have confirmed the relationship of MO with the performance improvement of organisations, and no study showed a negative impact of MO (Kumar et al., 2011; Liao et al., 2011). That is, although not necessarily a competitive differential, it has a positive impact on company performance.

Although, as mentioned earlier, the SSCs in the public sector were born with the NPM movement which stimulates the customer orientation, it is not clear in the literature whether the public sector SSCs are market-oriented. On the contrary, there is evidence (e.g. the cases of SSC failure mentioned above) that government and non-profit organisation's SSC are not market-oriented. Thus, a well-tested MO construct from Narver and Slater is applied here to investigate the extent to which public sector SSCs are market-oriented (Narver \& Slater, 1990).

\subsubsection{Performance}

As noted in Section 2.2.2, New Public Management, public sector organisations are less susceptible to competitive pressures and are financed by taxes and not by revenue. Nevertheless, due to the quest for the professionalisation in public management, there is a growing demand for more efficient public management (Denhardt \& Denhardt, 
2000; Gonzalez et al., 2013). This social pressure, which also demands greater transparency in public management, pushes these organisations (including the SSCs) towards high-performance work practices and greater efficiency. High-performance improvement is one of the main motivations for the deployment of public sector SSCs. Therefore, several mechanisms for controlling and reporting performance have been initiated (Sole \& Schiuma, 2010). Studies have shown that the use of performance monitoring systems improves the results of public service (Speklé \& Verbeeten, 2014).

The strong relationship between market orientation and successful business performance has been proven by numerous empirical studies; the more the organisation is focused on its market, competition, and customers, the better its performance (Hult \& Ketchen, 2001; Baker \& Sinkula, 1999; Kirca et al., 2005). Studies show that, in the case of non-profit organisations, this positive relationship is even more intense (Cano et al., 2004). For a non-profit organisation, being open to an external environment engenders greater responsiveness, innovation, and flexibility. As a result, the organisation is compelled to improve its systems, process, and performance (Deshpande \& Farley, 1998).

It is worth noting that $\mathrm{MO}$ is just one of numerous factors that affect the performance of a company. These include alignment of strategy to the business environment, organisational and managerial issues, the alignment of the structure with the strategy, and the degree of innovation in its products, processes, and services (Greenley, 1995; Han et al., 1998). It is even affected by entrepreneurship, and organisational learning (Hult \& Ketchen, 2001); as well as organisational responsiveness (Hult et al., 2005). However, despite the proven existence of these 
moderators between MO and performance, all studies have ratified the positive association between market orientation and performance.

Five conceptual lenses were extracted from the literature to analyse the construct performance: (a) goal orientation - checking if there are goals and strategies to achieve; (b) measurement system - assessing the quality of the performance verification mechanisms; (c) resources for performance measurement - which seeks to identify the dedication of resources to the performance monitoring; (d) performance - to assess the performance of the service operation; and (e) society results - which assesses the impact of the service operation to society (Sole \& Schiuma, 2010; Oliveira \& Roth, 2012; BouLlusar et al., 2009).

This section discussed six key elements to understand the factors that can influence the performance of an SSC (culture, leadership, resources, readiness for change, excellence, market orientation), and also the performance dimension itself. To study the interrelationship of these factors, and their contribution to the performance of an SSC, a conceptual model was designed, which is discussed in Section 2.6.1.

\subsubsection{The Proposed Conceptual Model}

In the previous sections, the literature analysed for understanding the current knowledge about the shared services centre (SSC) phenomenon and where it is placed in the context of management theory was discussed. From the exploitation of the existing work, it has been possible to identify the gaps and choose the research problem. Moreover, it was possible to find constructs and measurement scales developed and tested and elaborate the conceptual model to analyse the interrelations of the factors that influence the performance of SSCs. In this section, the conceptual model is presented, and focus is given to the assumptions made and the interaction of variables. 
Section 2.6.4.1 below shows how the model was designed, the literature that supports each model variable, and an overview of the relationship between the variables. A more detailed theoretical discussion of each model variable is found in Section 2.6.3, including the operational definition of each concept. Soon after the conceptual model background, Section 2.6.4.2 discusses the hypotheses formulated for the empirical test of the model.

\subsubsection{Conceptual Model Background}

The conceptual model of this research should help understand how the organisational elements Culture, Leadership, Resources, Readiness for Change, and Excellence contribute to the strategic direction Market Orientation and the outcome Performance of the SSC. For this, I developed the following in the model shown in Figure 2.1, that is based on constructs and measurement scales properly established, and solidly tested in previous studies by other researchers. As there are virtually no specific measurement scales for shared services centres, measurement scales used were derived primarily from quality, and service operations, whose sources are shown in Table 2.2 below. 
Table 2.2 Conceptual model variables and the corresponding support from the literature

\begin{tabular}{|c|c|c|}
\hline Dimensions & $\begin{array}{l}\text { Variables (constructs) } \\
\text { to be tested }\end{array}$ & $\begin{array}{l}\text { Source of construct/ measurement } \\
\text { scales: }\end{array}$ \\
\hline \multirow{10}{*}{$\begin{array}{l}\text { Organisational } \\
\text { elements }\end{array}$} & \multirow{3}{*}{ Culture } & Quality culture: Hietschold et al. (2014) \\
\hline & & Service culture: Oliveira \& Roth (2012) \\
\hline & & $\begin{array}{l}\text { Organisational Buy-in: Noble \& Mokwa } \\
\text { (1999) }\end{array}$ \\
\hline & Leadership & Bou-Llusar et al. (2009) \\
\hline & Resources & Evanschitzky (2007) \\
\hline & Readiness for change & Cinite et al. (2009) \\
\hline & \multirow{4}{*}{ Excellence } & Excellence: Sharma et al. (1990) \\
\hline & & Service quality: Camarero (2007) \\
\hline & & Benchmarking: Hietschold et al. (2014) \\
\hline & & $\begin{array}{l}\text { Service standards and Value } \\
\text { Orientation: Oliveira \& Roth (2012) }\end{array}$ \\
\hline Strategic direction & Market Orientation & Deshpande \& Farley (1996) \\
\hline \multirow{5}{*}{ Outcome } & \multirow{5}{*}{ Performance } & $\begin{array}{l}\text { Goal orientation: Sole \& Schiuma } \\
\text { (2010) }\end{array}$ \\
\hline & & $\begin{array}{l}\text { Measurement system: Oliveira \& Roth } \\
\text { (2012) }\end{array}$ \\
\hline & & $\begin{array}{l}\text { Resources for performance } \\
\text { measurement: Sole \& Schiuma (2010) }\end{array}$ \\
\hline & & Performance: Liu (2009) \\
\hline & & Society results: Bou-Llusar et al. (2009) \\
\hline
\end{tabular}

The method of development of the conceptual model is discussed in sections 2.6.1 and 2.6.2.

Figure 2.1 below shows the conceptual model designed and the relationship between the independent and dependent variables. Also, it illustrates the proposition generated from the literature review that the more organisational elements Culture, Leadership, Resources, Readiness for Change, and Excellence are developed regarding best practices and maturity, the greater the shared service centre market orientation, 
and better its performance. For example, a culture that stimulates the practices of the quality, and of service to the client, in which there are sponsorship and incentive for the continuous improvement, must contribute to a better performance of the organisation. In the same way, a leadership that sets the example of near relationship with the client, and that drives the officials in work as a team and organisational transformation, must contribute positively to the performance. Furthermore, the right available resources (e.g. financial, physical, human) and the capacity of readiness for the change, influence the performance. The latter ensures that there is no discontinuity in the company's productivity, during the changes. As for the excellence, the existence of direction to the value for the client and rigid standards of service quality, lead the company to a higher level of market orientation (client) and performance.

As discussed earlier in this chapter, the market orientation has been investigated and confirmed to be related to business performance. Therefore, the conceptual model below tried to verify the degree of market orientation of the SSC. Finally, the model-in a sub-item of Performance-considers that performance should reflect not only the financial dimension but also the results for the society, especially if we consider the SSCs from the public sector.

Figure 2.1 Conceptual model of factors influencing SSC performance

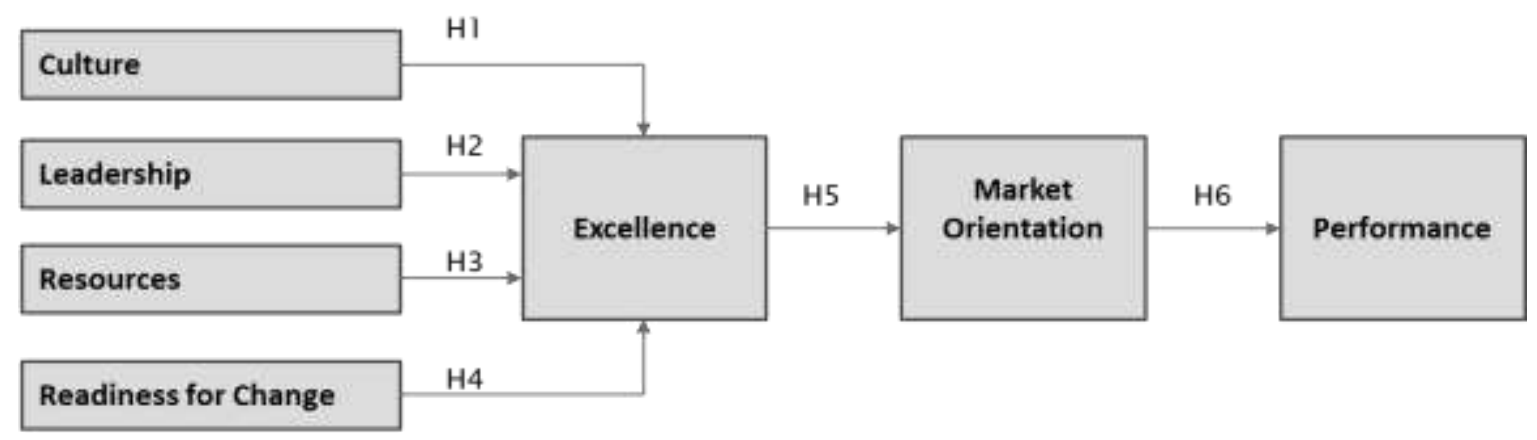


For the creation of this conceptual model, these seven variables were chosen since priority had been given to empirically tested constructs, which were investigating factors that contribute to the performance of service operations, which is the case of an SSC. These constructs were selected from the literature reviewed, considering mainly the extent of its practical application, that is, greater importance was given to those more established constructs by different empirical tests. Following these premises, it was possible to draw up a comprehensive and consistent conceptual model.

The following the hypotheses are contextualised and discussed to test the relationship between the variables.

\subsubsection{Conceptual Model and Research Hypotheses}

As seen above, the proposed conceptual model suggests that the independent variables of the model contribute to the performance of SSCs. To test the relationships of this proposition, six hypotheses were proposed (H1 to H6), as shown in Figure 2.1 above, and discussed below in specific subsection for each variable (2.6.4.2.1 to 2.6.4.2.6). According to Creswell (2013), in quantitative studies, hypotheses are used so that forecasts are made by the researcher on the relationships expected between the variables. The tests of the hypotheses use statistical methods and allow the investigator to make inferences about the population from the sample data. Note: the definitions of each concept/variable of the model are presented in Section 2.6.3.

\subsection{Culture}

Several studies have demonstrated the association between organisational culture and excellence in service, which has been confirmed by numerous works (Oliveira \& Roth, 2012; Mohammad Qawasmeh et al., 2013; Irani et al., 2004). To discuss organisational culture as a potential source of competitive advantage for a company, 
Barney (1991) puts it as an enabler of excellence. Hietschold et al. (2014) verified that the quality-oriented culture is essential for the success of total quality programs, which aim to achieve a level of excellence. Noble \& Mokwa (1999) proved that a culture where there is organisational buy-in contributes directly to the success of the implementation of a strategy (with an impact similar to the deployment of an SSC). Oliveira \& Roth (2012) have found that the culture of service relates positively to service orientation, with a propensity to deliver service excellence. These and other investigated studies have demonstrated that culture is related to excellence, and this was taken as probably also true in the case of SSC. Previous empirical studies found a positive relationship between culture and excellence in service (Hietschold et al., 2014; Noble \& Mokwa, 1999; Oliveira \& Roth, 2012), and this construct was taken to this research. Identifying the extent to which this is true in the context of public sector SSCs is one of the targets of this research.

Therefore,

H1: Culture relates positively to Excellence in SSCs

\subsection{Leadership}

The business excellence model by Kanji (1998), tested several times empirically, presents leadership as a protagonist in the generation of business excellence, in works originally developed in the field of quality. Another study, conducted by Prabhu \& Robson (2000), confirmed not only, that leadership is critical to the achievement of excellence in service, but that it is the determining factor in the differentiation of world class companies. Leadership has a prominent place in nearly all established models aimed at excellence in management, for example, the European Foundation for Quality Management (EFQM) and the Malcolm Baldrige National Quality Award (MBNQA) 
(Gouthier et al., 2012). Gouthier et al. (1994) while studying the main models of excellence in service, including the Johnston framework of the Deming Prize, found that leadership appears highlighted in these models, among the factors that enable excellence. Similarly, Bou-Llusar et al. (2009) studied four models of TQM of renowned authors, besides the EFQM and the MBNQA, and also proved the relation of causality between leadership and excellence empirically.

Therefore,

H2: Leadership relates positively to Excellence in SSCs

\subsection{Resources}

Theoretical approaches that study how organisational resources can bring competitive advantage for businesses, as the Resource-Based View (RBV), prove that resources are enablers for operational excellence (Barney, 1991; Fok-Yew \& Ahmad, 2014). Countless studies proved empirically that the resources connect positively with the operational excellence; in other words, for obtaining and sustaining competitive advantage and superior performance (Fok-Yew \& Ahmad, 2014), obtained through the company's resources. Operational excellence is not just about reducing costs and improving quality; it also involves the preparation and differentiated use of the resources (Fok-Yew \& Ahmad, 2014). According to Gouthier et al. (2012), operational excellence is the effective use of the company's resources and proper preparation of the processes leading to excellence. Evanschitzky (2007) also demonstrated empirically in his study that the resources (financial, physical, human, organisational, service-specific and intangible) relate positively to sustainable competitive advantage.

Therefore, 
H3: Resources relates positively to Excellence in SSCs

\subsection{Readiness for change}

Evans (2013, p. 437) argues that to develop and sustain performance excellence over time, it is essential that there is readiness for change. This causes companies to become stronger and more competitive, he said. To achieve Excellence, businesses must go through numerous changes, and be prepared for such changes is, therefore, a decisive factor. To respond to external changes, companies promote internal changes so that they can keep competitive (Brown \& Eisenhardt, 1997). Other authors such as Fok-Yew \& Ahmad (2014), Weeks et al. (2004) and Todnem By (2005), also have confirmed by empirical testing, the direct relationship between the readiness for change and operational excellence. Furthermore, it has been empirically proven that the preparation of the organisation's members is crucial to the success of the implementation of changes (Jones et al., 2005; Haffar et al., 2013). This positive relation between readiness for change and excellence having been proved in other areas of knowledge, is the starting point I need to check if such an observation is valid for the SSC.

Therefore,

H4: The greater the Readiness for change, the higher the level of the Excellence in SSCs

\subsection{Excellence}

Although studies on business excellence are not recent (Peters et al., 1982), just a few studies were found that evaluate the impact of the excellence on market orientation (Caruana et al., 1999). As we shall see in the following section in more detail, the abundant MO studies seek more to understand the consequences of being MO than its 
antecedents (Pulendran et al., 2000). For the development of the excellence part of the conceptual model, I sought support in four empirical studies: (a) attributes for

excellence (Sharma et al., 1990); (b) Service quality (Camarero, 2007); (c) Benchmarking (Hietschold et al., 2014); e (d) Service standards/ Value orientation (Noble \& Mokwa, 1999). From these studies, I extracted the constructs related to excellence, which allowed me to test the hypothesis $\mathrm{H} 5$ formulated below. The research of Caruana et al. (1999) with British service companies showed that there is a direct relationship between excellence and MO.

Therefore,

H5: Excellence relates positively to Market Orientation in SSCs

\subsection{Market orientation and Performance}

There are numerous studies of MO since the mid-20th century (Lear, 1963), with higher incidence from early 1990, and many of them research the impact of Market Orientation (MO) in business performance (for example, Pitt et al., 1996; Liao et al., 2011; Greenley, 1995; Hult et al., 2005; Narver et al., 2004; Kirca et al., 2005; Chang \& Chen, 1998). These studies analyse different complementary MO antecedents, as well as their consequences for the performance of organisations. For example, Narver et al. (2004) found that the higher the orientation of a company to the market, the greater the successful launch and performance of new products. Kirca et al. (2005) studied the antecedents and consequences of MO, concluding that manufacturing companies have higher relationship market orientation-performance than service companies. Finally, Chang \& Chen (1998) confirmed the positive relation between MO and performance, studying a broad conceptual model that contextualises the total quality. 
The conceptual model proposed in this document presents Performance as a dependent variable of MO. However, the performance of an enterprise is affected by other internal and external factors besides market orientation, for example, the regulations and the strategy. The organisational culture also can directly influence the performance, since according to Barney (1991) it can be a potential source of sustainable superior performance. Another study demonstrates that adopting the quality philosophy is significantly correlated to the firm performance in the long run (Hietschold et al., 2014). However, for the research of this thesis, the antecedent factor was deliberately restricted to MO. There is strong evidence that MO positively affects performance (Jaworski \& Kohli, 1993; Narver \& Slater, 1990; Slater \& Narver, 1994), so the conceptual model tested by the research of this thesis adopted Performance as the independent variable. It is possible that the SSC size influences the behaviour of the variables in relation to Performance, and this was also addressed to the scope of analysis to be performed. The existing studies reviewed show the positive relationship between MO and performance, and I did not find studies linking MO to the performance of Shared Services Centres.

Therefore,

H6: Market Orientation relates positively to Performance in SSCs

In this section, the conceptual model (factors influencing the performance of a SSC) was introduced and discussed, with a special focus on the hypotheses to be tested. An overview was offered on how each construct of the model was analysed and chosen from empirical research conducted by other researchers. Also, it sought to justify these choices with theoretical and empirical support. 


\subsection{Conclusion}

This chapter presented the literature reviewed for formulating the research question, develop the conceptual model that was one of the pillars of the field work, and support the analysis of the results and conclusions of the thesis. I tried to establish the emergence and the trajectory of the SSC in the private and public sectors, including its expansion in both sectors and referred to the major issues addressed by the literature. I then discussed the factors that may influence directly or indirectly the performance of SSC. As for the emergence of the SSC it was identified by my research that, although most authors regard the emergence of the SSC to the 1990s (e.g. Tomkinson, 2007; Schulman et al., 1999), the literature reviewed shows that they emerged much earlier, in the 1960s with the 'Rural Shared Service' in the USA (DeBlassie \& Ludeman, 1973; Wilson, 1970). Little research has been carried out on SSC in the public sector. Although a significant part of the literature reviewed here studied the SSC, their focus has been, for example, on themes like resources, competencies, governance, contracts and transaction costs, and the reasons for adopting (Fielt et al., 2014). As seen in this chapter, although they are present in most large businesses worldwide, the SSC is a very recent organisational phenomenon, mainly in the public sector. For this reason, the research on this subject is at the embryonic stage. We also saw that although the New Public Management-that, in essence, sought to introduce successful practices of private initiative in the public service, and one of these practices is the public service orientation to its customers (Hood, 1995b; Pollitt \& Summa, 1997)-has driven the SSC in the public sector, it is unclear if they are, in fact, market-oriented. On the contrary, there is evidence to suggest that the lack of market orientation has led to cases of failure. This gap was identified and addressed by the conceptual model developed from constructs already validated in other scholarly works as seen in the next chapter. Furthermore, in the conclusions 
chapter recommendations are made for the SSC public sector to become more marketoriented.

As also found in the literature reviewed, the adoption of the industry SSC model by the public sector resulted in numerous cases of success, but problems were also identified as well as significant financial losses (e.g. NCA, 2015; and Appendix A). This caught my attention, and I decided to explore the extent to which a single copy of the private sector SSC model, without due adaptation, may have caused such problems. For this, I have established the following research strategy: (a) first, to compare the performance of private sector SSC with public sector SSC and identify possible differences; (b) second, to obtain an explanation for these differences, based on current knowledge of management, for example, knowledge translation, organisational model, management model key differences between private and public sectors, and service design.

As many countries, including Brazil where I was born and live, tend greatly to expand the use of SSC in the public sector, I hope this study will be useful in contributing to accelerating the learning curve of the public managers embracing the SSC. The next chapter discusses the execution of the research, contextualizing Action Research with its mixed approach (quantitative and qualitative) applied in this inquiry, the accomplishment of the survey, the obtained results, and my apprenticeship along the project. 
CHAPTER 3

ACTION RESEARCH 


\subsection{Chapter Introduction}

With the central purpose of reaching the research aims, solving the research questions, and finding a good way to turn the knowledge developed by this research into action to improve the SSCs (especially from the public sector) performance, I adopted the Action Research (AR) approach. AR allowed me to, first, confirm the validity of the objectives and research questions of this study, which refer to relevant and concrete civil SSC problems. Secondly, identify, with the help of specialists/practitioners, appropriate solutions to the problems. Third, address measures and actions for the implementation of improvements in the adaptation of SSCs to the public-sector context, which can positively affect their performance. The specialists and practitioners were part of my Action Learning Set (ALS), as suggested by Coghlan \& Brannick (2014) and Greenwood \& Levin (2006). I have chosen to apply an ALS formed with outside experts because, as an independent consultant, I could not implement the knowledge developed by this thesis in my company, due to its small size. Thus, the ALS helped me draw up and format the knowledge for use in other organisations around the world. Broadly, with the AR approach, it was possible to understand what is happening with the SSCs, in the scope studied, and why these problems occur, as I explain in the following sections. The following, Section 3.2 provides the philosophical roots of this research.

\subsection{Research Philosophy}

The choice of a philosophical line to guide a social research is fundamental because besides clarifying how the researcher observes the social world, it helps to understand the own meaning of the developed knowledge. Easterby-Smith et al. (2008) point out that the philosophical orientation is important for three main reasons. First, it helps to define the general research strategy. Not only in defining what evidence will be required and how it will be obtained and interpreted, but also how the answers to the research 
questions will be provided. Second, the philosophical line helps the researcher identify which designs will be most suitable. For example, by clarifying the limitations of certain approaches and providing parameters for the choice of methodologies and methods, eliminating unnecessary efforts early in the study. Finally, it can help the researcher to be more creative and use approaches that (otherwise out of his or her knowledge), otherwise, would be ignored.

According to Johnson \& Onwuegbuzie (2004), It has been more than a hundred years since the proponents of the qualitative and quantitative paradigms have had heated debates that have led to the emergence of purists on both sides. Quantitative purists argue that social science research must be objective and that generalisations without regard to time, context, and to establish social laws are possible and desirable. On the other hand, qualitative purists (called interpretivists) reject what they call positivism and defend the superiority of the qualitative approaches, saying that in social events it is not possible to differentiate the cause of the effect and the subjective knower of knowledge completely. They also argue, unlike the positivists, that phenomena are susceptible to subjective interpretation. The two groups of purists understand that their views of the world are ideal, and think it is impossible to make them compatible (Johnson \& Onwuegbuzie, 2004). However, there are similar points in both approaches. For example, both quantitative and qualitative use empirical observations to solve research questions, and both take methodological care to avoid technical errors and preserve quality standards at acceptable levels.

Fortunately, many researchers propose pluralism as a solution to the philosophical positivism-interpretivism, and methodological quantitative-qualitative impasses (Saunders, 2012). After all, today's world is increasingly complex, dynamic and interdisciplinary, requiring methods that go beyond what a single tradition can offer 
(Easterby-Smith et al., 2008; Creswell, 2007). In the last thirty to forty years, pragmatism has been consolidated as a research philosophy. One of the central points of pragmatism, rooted in the works of American philosophers William James and John Dewey (EasterbySmith et al., 2008), is that in social studies, theories and concepts must come from people's practical experience. There must be a balance between observation and reflection on one side, and the abstract and concrete on the other. Many of the characteristics of pragmatism help to bridge the seemingly irreconcilable positivism and interpretivism, for example (Creswell, 2007): (a) the researchers are free to choose the techniques and methods that best meet the research objectives; (b) pragmatists do not view the world as a single unit, which in mixed approaches corresponds, as an illustration, to the use of various methods of data collection (quantitative and qualitative); and (c) pragmatists believe in the existence of an external world, but as well in a world within the people's mind. Pragmatism also enables the researcher to define how to make the mix of approaches more effective.

Action Research is rooted in pragmatism. Greenwood (2007), in discussing his proposal for 'pragmatic action research,' argues that Action Research is anchored in pragmatism and it was this worldview that led to the development of the multidisciplinarity of AR. He also understands that research participants should be free to choose the theories, methods, and techniques from social sciences and humanities. To give more example of scholars linking AR to pragmatism, Stringer (2013) makes a wideranging discussion of how the essence of pragmatism is inextricably present in the design and practices of the AR. He also argues there is space in the AR for the use of methods and techniques from the traditional science. Another aspect to be highlighted by adopting the philosophy of pragmatism is the concept of triangulation (Webb, 1989) that suggests the application of different perspectives and methods, which complement 
each other and lead to a more complete and better understanding of the subject under study.

The research for this thesis was conducted within the philosophy of pragmatism and used the design explanatory sequential mixed method (Creswell \& Clark, 2007). This method assumes that the quantitative approach is first performed, the results validated and analysed. The qualitative approach is then followed to complement the interpretation and to evidence the practical application of the initial results, as discussed in the following sections.

\subsection{Research Design}

Under an Action Research approach, with roots in the pragmatic philosophy and general systems theory (Greenwood \& Levin, 2006), the design of this study is descriptive, with cross-sectional survey, using mixed quantitative and qualitative methods. After investigation and study of the (scarce) existing literature about the Shared Services Centres (mainly in the public sector), as well as the literature on public service and service operations, it has been possible to set up the theme, and the problem of research referred to in the previous section. During the literature review, it became evident that for the empirical investigation of this thesis, it would be essential to apply an approach that could deal with a complex context of a problem with multiple faces. This required an approach that would work with mixed methods, to be carried out quantitative and qualitative analyses of data on the phenomenon under study. It is common when it comes to management research, that a problem requires more than one approach and eclectic designs to be studied and solved (Easterby-Smith et al., 2008; Johnson \& Onwuegbuzie, 2004; Creswell \& Clark, 2007). This is the context of the research for this thesis, which demanded a mixed method because the quantitative 
approach (developed applying a survey) was necessary to ensure a consistent study of the interrelationship of variables of the conceptual model proposed, seen in the previous chapter.

In this thesis, it was used a cross-sectional survey, applied to 146 respondents from different shared services centres, public and private, in countries such as Brazil, United States, the United Kingdom, and like. To collect the quantitative data, a questionnaire was developed in line with the inferential survey concept, according to which, it first established the conceptual model and the relationship between the variables and concepts (Easterby-Smith et al., 2008) to be then tested by application of that instrument. One of the main concerns with the use of questionnaires for data collection is the quality of the items applied to measure the variables under study, the scales of measurement (Easterby-Smith et al., 2008). To minimize this problem, I used, as detailed in Section 3.8 Questionnaire and Measurement Scales, peer-reviewed scales widely approved, developed and empirically tested by other researchers. This way, I could bring to the study the safety of the items presented in the questionnaire and measure the variables of the conceptual model (discussed in the previous chapter).

On the other hand, the polishing and preparation for the practical application of the knowledge produced by the quantitative approach required a qualitative approach. To do that I used the insights and experience of experts in Shared Services Centres (SSCs) and Public Service, through an Action Learning Set (Pedler, 2012), mentioned in the previous section. As shown below, experts in public services were heard because, although there were surveyed SSCs from the public sector, and industry, the priority target for applying the research results are the SSCs of governments-in all spheres of activity, federal, state, regional and municipal levels, as explained in Chapter 1. 
The following are presented sections What is Action Research and How it Emerged, How This Study Applied the Action Research, Method of Survey, Sources of Data and Communication Methods, Questionnaire and Measurement Scales, Administration of the Research, Statistical Approaches/Data Analysis, Quantitative Action Research, Qualitative Action Research, Results Obtained With the Action Learning Set, and My Learning With the Action Learning Set.

\subsection{What is Action Research and How It Emerged}

According to Reason \& Bradbury (2001), Action Research (AR) is a set of practices to apply knowledge and solve a given problem. The definition of McNeill \& Chapman (2005) perhaps is even more suitable for this research because according to them, AR is a systematic way for researchers and practitioners to conduct research which is designed to generate practical results, which are used to improve specific aspects of the context of the practitioners. AR offers to the practitioners the opportunity to study their problems scientifically (Greenwood \& Levin, 2006), giving prominence to the practical relevance of the research. AR helps cover the gap between theory and practice, combining ideas and actions necessary to promote some improvement for individuals, groups or entire communities. Although it combines action and research, it is not a methodology, but a process, participatory and democratic, which creates positive change in greater or lesser extent (Coghlan \& Brannick, 2014). In addition, Greenwood \& Levin (2006) pointed out that AR also provides apprenticeship to the participants of the process.

The emergence of the AR is attributed to the studies of Kurt Lewin, one of the fathers of social psychology in the 1940s (Barton et al., 2009; Susman \& Evered, 1978). However, the development of AR also goes by the works of Brazilian researcher Paulo 
Freire on consciousness-raising, and has even more ancient roots, as Marxists' studies and the Aristotelian philosophy (Coghlan \& Brannick, 2014). While it may have Marxist influence, the AR can be applied independently of any ideology. The basic idea of AR, according to Coghlan \& Brannick (2014), is to apply a scientific approach to study and find solutions to relevant organisational or social problems, together with the people who experience such problems. In the case of this thesis, AR was applied to better understand the problem and to submit the findings of the survey carried out, to specialists in public service and SSCs managers to assess its relevance and find ways to operationalise them. Barton et al. (2009) argue that AR can be developed in a two-step cycle, the first step of diagnostic when the problem is identified and analysed, and the interpretations (hypotheses) are formulated to explain it. The second step, when the hypotheses are tested, preferably in a real social situation. The study of this thesis was developed observing these two phases. The problem was identified and diagnosed through the qualitative and quantitative survey, and the test of the findings and preliminary interpretations was performed by the Action Learning Set. Furthermore, the ALS helped to provide a practical contribution applicable to the SSCs. The constitution of the ALS is detailed below in Section 3.13.1.

\subsection{How this Study Applied the Action Research}

As informed above, the empirical research conducted for this thesis used a mixed design that combined quantitative and qualitative approaches. The quantitative approach, discussed in Section 3.11, was used to test the conceptual model proposed by this thesis. Through this model, the factors that can influence the performance of SSCs were tested statistically. The qualitative approach, presented and discussed in Section 3.12, was applied to understand if there are significant differences between public and private SSCs. The results of both approaches, qualitative and quantitative, were then 
subjected to discussion and analysis of a team of seven experts, through an Action Learning Set, further discussed below. Therefore, it was possible to identify and format knowledge generated by this study that may help to improve the performance of public SSCs.

\subsection{Method of Survey}

\subsubsection{Sampling Approach and Considerations}

The three classical approaches to the definition of a sample (sampling strategy) to be researched are secondary data, probability sampling survey and population survey (Rungtusanatham et al., 2003; Easterby-Smith et al., 2008; Dillman et al., 2014). As this is an unmatched survey of a recent phenomenon, yet there is not much available secondary data, much less to the theme and the research questions of interest of this thesis. The population of SSCs (discussed below) is not yet known, which prevents the use of probability methods and population survey (Dillman et al., 2014). Therefore, I decided to adopt a non-probabilistic method (non-probability sampling), specifically, the convenience sampling. The convenience sampling was embraced because of my access to groups of SSC managers from countries like Brazil, Canada, UK, and the USA, among others. My access to SSC managers comes from three complementary sources: (a) my participation in SSC international conferences in the USA, with the presence of hundreds of managers; (b) contact details of SSC public sector managers of UK I have collected through Internet search; and (c) about 20 groups specialised in SSC on LinkedIn, which totalled in March 2016 more than 80,000 members. For example, HR Shared Services Network, with more than ten thousand members and Global Shared Services Executives, with more than seven thousand. The approach to these managers is detailed in the following subsections. 


\subsubsection{Population}

It was not found a total number of Shared Services Centres in the world, nor in any region, not even estimated. However, there is research conducted by a management consulting firm, focusing on the private sector, which illustrates the potential reach of the population of SSCs. Accenture (2013) reports that more than $75 \%$ of the 500 companies listed by Fortune magazine have SSC. Searle (2014), citing the same base of Fortune, says that companies adopting SSC in that group range $90 \%$. Thus, though there are not real numbers as for the total of SSC, it is possible to have an idea of the dimension and representativeness of the SSCs in the industry. As for the public sector, total numbers were also not found, but the relevance of the SSCs in governments like Canada, UK, and the USA, can be verified by their massive inclusion in their policies of reducing costs and improving service to the citizen (Schwarz, 2014; Tomkinson, 2007). Furthermore, the Organisation for Economic Co-operation and Development (OECD) has recommended that its members adopt the SSC as a way of making the public management more efficient in their countries (OECD, 2010).

\subsubsection{Sampling Process}

To test the hypotheses of the conceptual model by the appropriate public and verify the relationship between variables, were followed the steps of sampling process in Figure 3.1 below. 
Figure 3.1 Sampling Process adapted from Kalyan Acharjya (2016)

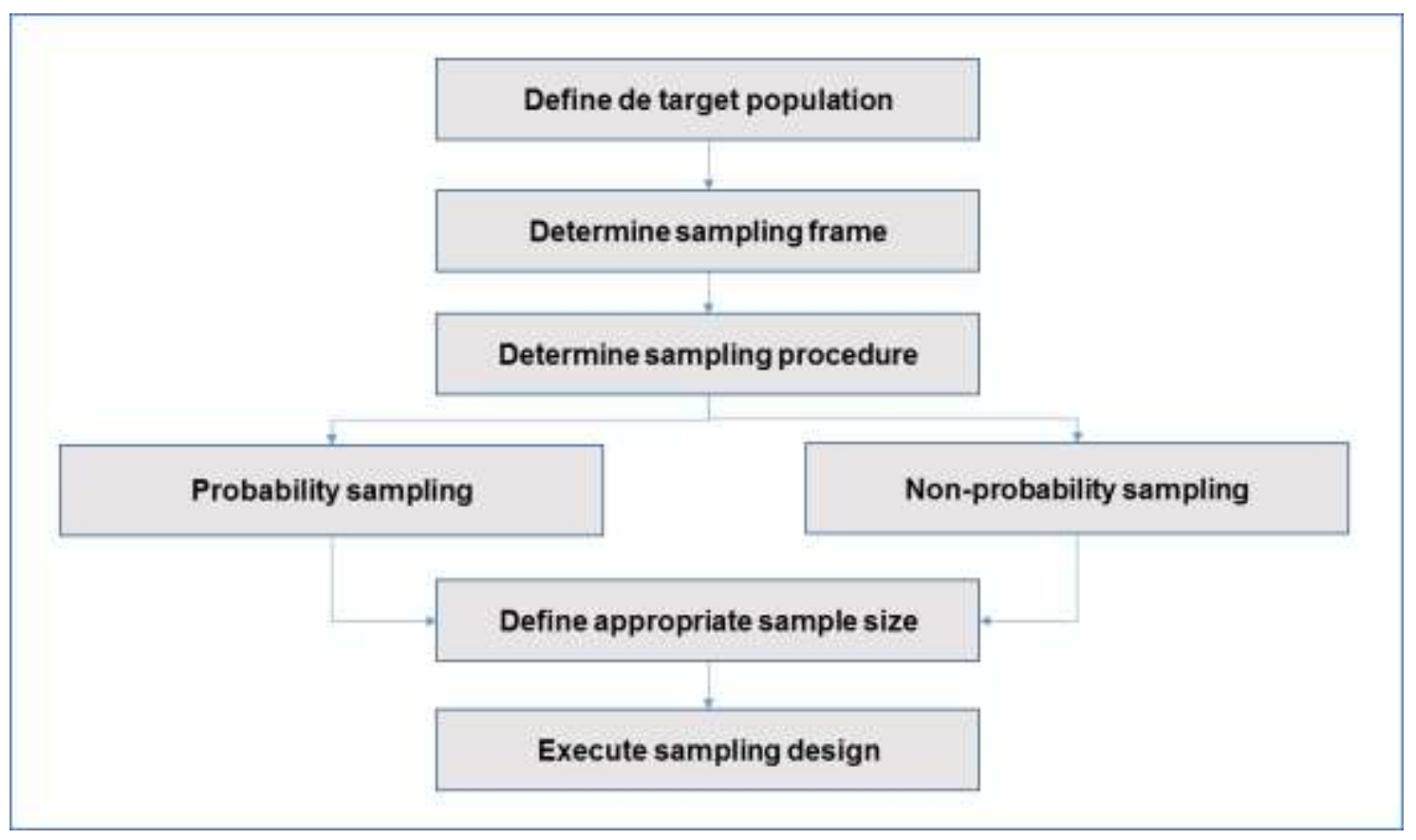

Figure 3.1 above presents the essential steps of sampling to ensure that the data collected are free of four major types of errors, according to Forza (2002) and Malhotra \& Grover (1998): (1) sampling error, which prevents the results from being generalised; (2) measurement error, arising from the use of measurement scales not compatible with components of the conceptual model; (3) statistical conclusion error, which leads to error in the determination between existing or not a relationship between the investigated variables; and (4) internal validity error, what takes place when the rival hypothesis can explain the relationships.

In step 'Define the target population' identifies the population to be considered in the survey, which will enable Determine the sampling frame in step two when potential respondents are identified. Completing this activity, it is possible to go to the next step to Determine sampling procedure that will ensure that the correct profile of respondents participates in the research. It goes on, then, to the choice of a method probabilistic or non-probabilistic, depending on the context of the project, on the maturity of the 
investigated phenomenon, on the existing population, and on the availability of respondents. In Define appropriate sample size established the required sample size to have representation and, in the last step Execute, sampling design defined the proceedings for deployment of the procedures that will lead to having the proper sampling frame, discussed below.

\subsubsection{Sampling Frame}

The sampling frame is the set of all those who can be chosen to participate in a survey, or a subset of the total population (Easterby-Smith et al., 2008; Dillman et al., 2014). Initially, I wanted to survey just the SSCs of the English public sector, using a list of 226 SSC managers that I prepared from a search on the Internet. However, after two months between the invitation sent by email to 226 managers to participate in the study, several follow-up contacts and only 26 responses (11.5\%), I decided with my supervisor to include the private sector SSCs from the UK and other countries. The response rate itself had been not so bad, because usually, the rate of responses on the Internet is smaller than those of classical methods, such as mail and telephone (Dillman et al., 2014), but with only 26 cases, I wouldn't have enough to ensure representativeness and accuracy, and to ensure precision in the results and recommendations (Easterby-Smith et al., 2008). After that decision with my supervisor, I adopted a new approach of survey known as networking sampling (Dillman et al., 2014), and extended the sampling frame to approximately 80,000 members of LinkedIn groups specialised in SSC and about 50 contacts details of managers of SSC from the US and Canada. With these measures, it was possible getting more 120 responses totalling 146 cases, an appropriate number for testing the conceptual models from a statistical standpoint. To have adequate sample size is important in any empirical study to be able to make inferences regarding the population. In empirical research with non-probability sampling, it is necessary a 
sample size greater than 100 cases (Hair, 1998; MacCallum et al., 1999), and the total of 146 cases in this study qualify it as for the size of the sample.

\subsubsection{Sampling Unit}

Though not always present in empirical research, the prior specification of the unit of analysis, that is, who or what is being studied, is a relevant factor within the sampling strategy (Rungtusanatham et al., 2003). In other words, the unit of analysis is the entity which is the basis of any sample outside-in (Easterby-Smith et al., 2008). It is from it that the data will be collected and analysed. The unit of analysis of my thesis is the shared services centre (SSC). That is, an entity providing services to other components of the organisation, or even to the external customer, or citizen in the case of the public sector, in unique and innovative rules (characteristics of an SSC), as discussed in Section 3.3. Research Design. There has been no geographic or industry restriction for the SSC to be investigated. Moreover, there is no concern to previous statistic procedures for selection of units to be surveyed due to, as mentioned above, the non-probability sampling nature of this study. As for the persons who will respond to the questionnaire, with this sampling unit definition, are discarded, for example, potential respondents who do not have enough degree of knowledge of the SSC target of the survey.

\subsection{Sources of Data and Communication Methods}

This section discusses the aspects related to data sources and communication methods for obtaining quantitative data needed to test the conceptual model.

\subsubsection{Sources of Data}

A researcher can collect data for a survey from secondary sources, i.e., data produced by other researchers, or primary sources when they are generated by the interested party. As mentioned in Section 3.6. Method of Survey, I had to opt for primary 
data due to the SSC theme practically do not have secondary data available, at least for this study. The primary sources of data can be accessed mainly through observation, interviews, or Web-based surveys (Dillman et al., 2014). The use of the Internet for realising inquiries has been growing substantially in the last years, making possible a significant decrease of costs and terms (Dillman et al., 2014; Easterby-Smith et al., 2008) and the Web-based survey was the way chosen by me for the inquiry driven in this thesis. More and more people from all over the world use the Internet, and the Internet penetration rate (percentage of the population with access to the Internet) grows every year. In some countries already exceeds $90 \%$, the case of the UK, for example, with 91.6\%, and Netherlands with 93.2\%. Other countries like the USA (87.4\%), Brazil (57.6\%) and Canada (87.1\%) also have satisfactory Internet penetration rate to conduct a survey via the Internet (Translated, 2016). Concerning the use of the Internet, the Web survey applied in this research is classified by Couper (2000) as List-based samples of high-coverage populations. This type of Web survey begins with a sample frame or list and inviting participants by email, using access controls to prevent more than one response of the same respondent. More details about the sampling frame considered to getting direct data are presented in the previous Section 3.6 Method of Survey, subsection Sampling frame.

\subsubsection{Communication Methods}

The chosen method of inquiry Web List-based samples of high-coverage populations (Couper, 2000), mentioned above, resulted in a prior communication by email to the potential participants of the survey, inviting them for the inquiry and giving a link to the online questionnaire to those who confirmed the willingness to taking part. They all were briefed on the voluntary nature of participation and anonymity and confidentiality in treating the data provided. The online questionnaire was initially 
hosted on the platform EUSurvey of the European Commission's ISA Program and later, with the extension of the sampling frame for other countries, the American platform SurveyGizmo. The contact with the invitation to engage in the survey took place in two different ways, depending on the audience.

Public 1, managers and other professionals with knowledge on SSC, with whom I had direct contact. They were sent individual emails, inviting them to join in. They were also sent two follow-up emails to non-respondents. Those who responded affirmatively received a new email with more details about the study and a link to the online questionnaire. The questionnaire for this group did not have pre-qualifying questions, and the respondents were already directed straight to the main sections of the questionnaire.

Public 2, participants of LinkedIn groups specialised in SSC. They were invited to take part in the inquiry, mostly through general, impersonal notice, and in some groups, through an individual note with reinforcement of the moderator of the group. The link for the online questionnaire was already supplied in the first message. The questionnaire for this group had pre-qualifying questions, and they were only directed to the main questions those respondents who had knowledge on SSC. In the next section, the rational and the content of the questionnaire are discussed in detail.

\subsection{Questionnaire and Measurement Scales}

A questionnaire is a body of questions used to obtain the desired information from the respondents (Malhotra, 2006; Dillman et al., 2014), i.e. an instrument to collect written data. Its use permits to transform qualitative information into quantitative that can receive statistical treatment, allowing, for example, to validate the suggested conceptual model and hypotheses of this thesis, through appropriate measurement 
scales. There are many risks involved in the design of measurement scales, which can lead to measurement errors (Dillman et al., 2014), and distortion of the results, and it is also necessary considerable time and numerous practical applications of a scale so that it can be considered mature and helpful. For this reason, I decided to use existing measurement scales, previously tested. In Section 2.6 Conceptual Model, the sources of the scales used to measure the constructs of the conceptual model of this research are presented, and the individual scales are discussed in Section 3.11. Quantitative Action Research, and the Appendix B presents the questionnaire applied. The sections below present, pragmatically, the most relevant concepts and practices used in the preparation of the questionnaire, considering the context and specific approach of this survey, and covering the entire questionnaire cycle from the design phase up to the pre-test and validation.

\subsubsection{Information Sought}

Knowing what information is required and for what purpose it will be used is critical to the design of a questionnaire. The central aim of this data collection instrument, as mentioned above, is the validation of the conceptual model proposed by this thesis, which obviously, was developed before the questionnaire. For the constructs used in the proposed conceptual model already existed measurement scales adequately tested. Thus, the definition of necessary data was quite easy, although the set of constructs and their interrelationship have been subjected to continuous attention, mainly to ensure consistency and coherence of the model. I sought at this step to make sure the questionnaire accurately reflects the scales of measurement of each construct, previously tested by their authors, as reported in Section 2.6. Conceptual Model. I used only one author by construct not to invalidate the previous tests, due to possible conflicts. Other information, such as the profile of the SSC and the respondent, were also 
included in the questionnaire to ensure the adherence of each case to the sampling strategy defined. Finally, an open question was added to understand the view of the respondent about the main differences between the SSC of the public sector and the private sector one.

\subsubsection{Type of Questionnaire and Method of Administration}

The developed questionnaire is a Web-based survey type, to be completed by the respondents themselves in their computers after they had been invited by e-mail and directed to a link to the online questionnaire. Because of having been created under the "Web List-based samples of high-coverage populations (Couper, 2000)," the questionnaire was accessed only by persons previously invited. Lowering the risk of being filled out by individuals with inappropriate profile. Even so, pre-qualification measures were adopted (see the previous section) to sources of potential respondents with diverse profiles, as the LinkedIn specialised SSC groups.

\subsubsection{Individual Question Content}

According to Malhotra (2006), the main concerns regarding the individual content of the questions are: if the question is really necessary; the need for more than one question to obtain the information; and that the question is not worded in such a way as to contain two questions (double-barrelled question), hinder the respondent to be objective in his or her response. Gil (2002) argues that one should not include questions whose answers can be obtained from another more accurate source; the question should not suggest answers; the question must not cause defensive responses; avoid the use of stereotypical words; and that the question should not invade the privacy of the respondent. All these recommendations were considered when reviewing each of the 
questions. Recalling, the questions used were obtained from previously designed and tested measuring scales.

\subsubsection{Forms of Response}

In social research, there are many ways to get answers such as, for example, checklist, yes or no, ranking, and Likert-type scales (Seashore \& Taber, 1975), which allow obtaining primary data, permitting the consolidation of data for comparison between groups. The research of this thesis held the scales originally developed in the Likert format, ranging from 1 - Strongly Disagree up to 7 - Strongly Agree (see Figure 3.2 below). This form of response, combined with other survey design features, enabled an average fill under 20 minutes thanks to the consistent and pleasant layout and informative opening text and closure, as recommended by Dillman et al. (2014).

Figure 3.2 Example of the Likert scale applied to the questionnaire

\section{SSC CULTURE}

In answering the following questions, please focus on your SSC organization.

SSC CULTURE - Quality culture

\begin{tabular}{|l|c|c|c|c|c|c|c|}
\hline & $\begin{array}{c}\text { Strongly } \\
\text { Disagree } \\
1\end{array}$ & 2 & 3 & 4 & 5 & 6 & $\begin{array}{c}\text { Strongly } \\
\text { Agree }\end{array}$ \\
\hline $\begin{array}{l}\text { Continuous quality improvement is part of } \\
\text { all employees' responsibility rather than of } \\
\text { the quality department alone. }\end{array}$ & 0 & 0 & 0 & 0 & 0 & 0 & 0 \\
\hline $\begin{array}{l}\text { Our SSC establishes long-term goals } \\
\text { related to quality. }\end{array}$ & 0 & 0 & 0 & 0 & 0 & 0 & 0 \\
\hline $\begin{array}{l}\text { Our SSC develops an organization-wide } \\
\text { culture of quality. }\end{array}$ & & 0 & 0 & 0 & 0 & 0 & 0 \\
\hline
\end{tabular}

\subsubsection{Question Wording}

Most of the questionnaire questions are derived from the predefined measurement scales, as mentioned above, so the wording of them could be used almost in full in the 
English questionnaire. However, translation into Portuguese was necessary for Brazilian respondents, and it adopted the following recommendations to review the writing of each question in both languages. Key Recommendations of Malhotra (2006) for writing questions: using simple words; avoid ambiguous terms; avoid assumptions; use positive and negative statements (control questions to ensure the quality of responses). When analysing the data, it is necessary to remember to reverse the negative questions. To these recommendations, Gil (2002) adds that it should provide adequate instructions for completing the questions. As Portuguese is my native language, it was not adopted the back-translation procedure.

\subsubsection{Question Sequence}

Having written questions, it is necessary to define the best sequence, which will promote more consistent responses from the participants. I took some precautions recommended by Gil (2002) e Malhotra (2006): pre-qualification questions before the questionnaire itself; start the questionnaire with the simpler questions and finish with the most complex; establish logical sense between the questions; disperse the questions that might cause some 'contagion'; questions for general information at the end. Furthermore, Dillman et al. (2014) add: choose the appropriate format of the question; check if the question is suitable for the respondent; check if the question is technically accurate; use the smallest number of words possible. In the research of this thesis, the main questions were grouped by the constructs of the conceptual model.

\subsubsection{Questionnaire Form Characteristics}

Care should be taken about the visual presentation of the questionnaire, to facilitate its fill. In General, studies have shown that as the wording of questions influences the quality of the responses, survey instrument design also contributes to 
both the research conducted by an interviewer as in self-administered surveys (Couper et al., 2001; Couper, 2000). The platforms EUSurvey and SurveyGizmo have resources of the last generation that allowed the drawing of a quite organised and visually attractive questionnaire, reducing the possibility of mistakes and lack of motivation among respondents.

\subsubsection{Re-examination and Revision of the Questionnaire}

Before doing the pre-test of the questionnaire, I did a full review, a few days after it was ready, in search of any error, possible difficulty in filling, or some situation that prevented the achievement of the final goals of data collection. Even before the pre-test, I submitted the questionnaire to two people experienced in scientific research, to know if they would have some difficulty in understanding or completing the questions, and it has been approved.

\subsubsection{Questionnaire Pretesting}

The first step of this stage is to select individuals of the target group and to apply the questionnaire, checking the time they took to answer and eventual doubts, difficulties or comments they may have. The pre-test of the questionnaire for this study was conducted with six respondents in the sample of potential participants, to verify, in particular, the following points (Gil, 2002): clarity and precision of the terms; the amount of questions; the form of the questions; the order of questions; and any opportunity to improve the introduction of the questionnaire. In the pre-test analysis I sought to check if all questions were answered correctly, if the answers were not showing any difficulty of understanding, if the open answers were susceptible to categorisation, and if all that could entail in the inadequacy of the questionnaire as an instrument of data collection (Gil, 2002; Dillman et al., 2014). 


\subsection{Administration of the Research}

Two important aspects related to the administration of the research are treated here. The first regarding the management of the research project discussed below in the topic Administrative plan. The second concerning the execution of the survey and the role of the respondents discussed in Response matters.

\subsubsection{Administrative Plan}

The management of the research involved issues such as the organisation of the project, the control of its progress and quality control. In the project organisation, I have developed a project timeline, and a milestone plan has been agreed with the first supervisor. Thus, it was possible to anticipate the main products of the project, as well as the activities for the preparation of each one, in addition to the time necessary for this. For tracking progress, fortnightly meetings have been set up with the first supervisor, where doubts were clarified, and feedback provided. As quality control, the partial products generated throughout the work were submitted to supervisors for review and getting suggestions for improvement. Besides taking care of these issues, the research management gave attention to compliance with ethical standards set by the Research Ethics Committee. For example, the original data collection records were exclusively in my power, password-protected and have been transformed into work records, taking care to give each of them a numeric code, so not to associate them with the respondents or their organisations. Also in the research management were defined the skills needed for successful work and achievement of goals as, for example, the appropriate profile of the participants of the Action Learning Set, as discussed in this chapter. 


\subsubsection{Response Matters}

In this subsection, two topics are covered, the quality of participation and the provided answers, and ethical issues related to the relationship with the actual and potential respondents. Concerning the quality, usually are referred to more often two main sources of errors in surveys, (A) non-response error, and (B) response error. (A) Non-response error, according to Dillman et al. (2014), occurs when the characteristics of the respondents are different from the characteristics of those who no longer respond, to such a degree that can distort the survey results. This checking is made difficult since we have no access to the data of those which did not answer, but it can be tested, to a certain extent, by comparing the data (demographic and population not) of those who responded last, with the answers of those who responded early in the study. The logic of this test is that those who answered last are likened more to those who did not respond, and can give a good idea about the occurrence or not of non-response error. This test was conducted using the extrapolation method and is described in Section 3.11 Quantitative Action Research. To minimize non-response error Easterby-Smith et al. (2008) recommend adopting techniques such as: make the task quick and easy for the respondent; explain the objectives clearly, to understand the value of the activity; ensure confidentiality and anonymity; and send reminders. All these techniques were used in this research. (B) Response error occurs when the respondent provides inaccurate or wrong answers while putting his answers in the questionnaire. To avoid this type of mistake, special care was taken in the design of the questionnaire (see Section 3.8) and in the general and specific communication on each question, to minimize the chance of the respondent error in responding. Another quality assurance feature was the use of statistical methods to confirm the consistency of the data collection instrument, before testing the hypotheses. 
Regarding the ethical standards, all respondents were informed of the voluntary nature of their participation, as well as confidentiality and anonymity in the treatment of the data collected, and that neither they nor their organisations would be reported individually in the publications relating to the research. Also, it was provided to them the email from the representative of the Research Ethics Committee, responsible for resolving any doubts about the legitimacy of the research.

\subsection{Statistical Approaches/Data Analysis}

The quality of the measurement scales used in data collection instruments is essential, especially in research seeking to test the relationship between constructs (Rungtusanatham et al., 2003). When it comes to validation of measurement scales and constructs, two main assessments are used, reliability and validity. According to Nunnally \& Bernstein (1994), reliability means stability over time, and validity is the scientific utility of a scale to measure what it intends to measure. The reliability of the scales used in the research of this thesis was evaluated through: (A) exploratory factor analysis and (B) Cronbach's alpha, and validity of each construct was analysed by (C) Pearson's correlation, and (D) regression (multicollinearity). (A) exploratory factor analysis is defined as a set of multivariate techniques that aims to find the underlying structure in an array of data and determine the number and the nature of the latent variables (factors) that best represent a set of observed variables (Brown, 2015). (B) The Cronbach's alpha was presented by Lee J. Cronbach in 1951 to estimate the reliability of a questionnaire used in a survey. Alpha measures the correlation between responses to a questionnaire, through the analysis of the profile of the answers given by the respondents. This is an average correlation between questions. Given that all the items of a questionnaire use the same scale of measurement, the coefficient $\alpha$ is calculated from the variance of individual items and the variance of the sum of the items 
of each evaluator (Rego et al., 2010). (C) The Pearson's correlation coefficient is a measure of the degree of linear relationship between two quantitative variables. This coefficient varies between -1 and 1 . The value zero $(0)$ means that there is no linear relationship, a value of 1 indicates a perfect linear relationship, and the value of -1 indicates a perfect but also an inverse linear relationship, namely when one variable increases the other decreases (Statstutor, 2016). (D) Multicollinearity in regression is a condition that occurs when some predictor variables in the model are correlated with other predictors. The strong multicollinearity is problematic because it may increase the variance of the regression coefficients, making them unstable (Williams, 2016). The application, results and discussion of these techniques can be found in Section 3.11 Quantitative Action Research.

It is presented in the next section, a detailed view of the established method for the quantitative part of the research. For example, the definition, execution, and analysis of the empirical study to test and validate the conceptual model, developed to confirm the hypotheses and assumptions about the factors that influence the degree of market orientation and performance of an SSC.

\subsection{Quantitative Action Research}

This section brings a description and analysis of data collected through a questionnaire applied to a sample of 146 shared service centres, from both public sector and industry of several countries, as discussed below. First, there's the characterisation and description of the sample. Next, are presented the factor analyses carried out with the objective of simplification of the constructs of the conceptual model, preparing it in this way for the subsequent analyses. After that, it explores the Pearson correlation method used to verify the association between the variables tested, and the results are 
discussed. It then passes to the explanation of the multiple regression analysis and what was found. Finally, it presents the result of the validation of the hypotheses tested empirically through the survey conducted, and the results of a t-test performed with all variables. To close the chapter, a summary of key findings and highlight points is offered. The interpretation and discussion of the results presented here are found in Chapter 4, and the qualitative part of the survey (an open-ended question about possible differences between SSCs from the public sector and industry) is presented in section 3.12 Qualitative Action Research.

\subsubsection{Descriptives}

The information presented below aim to characterise the sample (146 cases) of the population of SSCs.

\subsubsection{Sample characteristics}

The sample consists of 146 cases each corresponding to an SSC. It is reasonable to assume that each case roughly corresponds to a specific SSC, since, although it was optional, 110 respondents from a total of 146 (that is, $75 \%$ of participants), reported the names of the SSCs and it was possible to verify that in these cases, there is one reply per SSC. The other did not mention the name of the SSC, possibly for reasons of confidentiality. We received about 180 completed questionnaires that after screening resulted in 146 questionnaires suitable for the research. This sample of 146 cases has the following main characteristics:

As for the size, as shown in Figure 3.3 below, slightly over half (50.7\%) or 74 of the SSCs has more than 100 employees, which is reasonable because large organisations typically adopt the SSC as a solution to their support services, such as administrative, financial, HR services and ICT, among other. 
Figure 3.3 Size of the SSC based on the number of employees

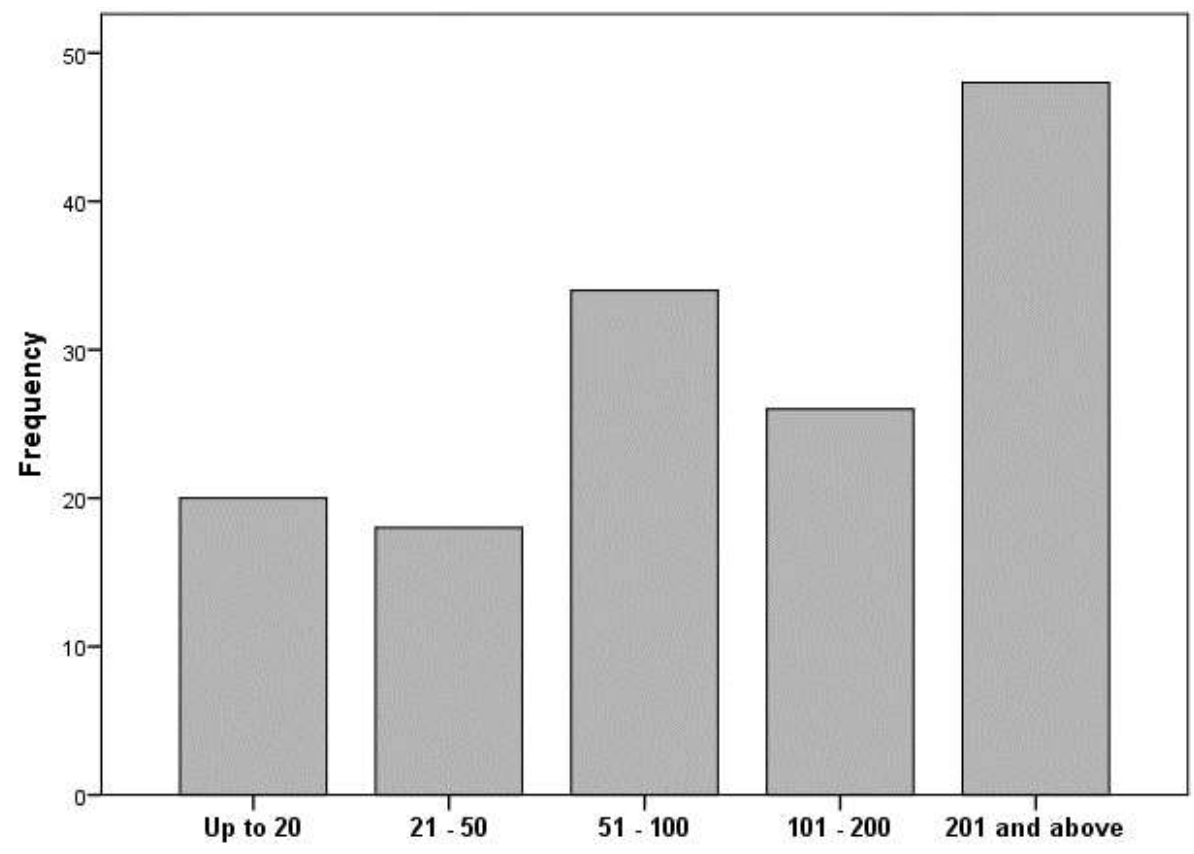

Regarding the stage of development (see Table 3.1 below), the majority (73.3\%) is at a time of growth or transformation. On the other hand, it caught my eye that only two of the evaluated SSCs are in a moment of deployment or post-deployment (Launching). This could denote a deceleration in new SSCs deployments. Also, 34 respondents report that the SSC is in design or revision of the initial design (Visioning). It was reported that one of the three SSC classified as other recently shut down, and the services provided by him returned to organisational areas of origin. 
Table 3.1 Stage of SSC development

\begin{tabular}{|c|c|c|c|c|c|}
\hline & & Frequency & Percent & Valid Percent & $\begin{array}{c}\text { Cumulative } \\
\text { Percent }\end{array}$ \\
\hline \multirow[t]{6}{*}{ Valid } & 0. Other & 3 & 2.1 & 2.1 & 2.1 \\
\hline & 1. Visioning & 34 & 23.3 & 23.3 & 25.3 \\
\hline & 2. Launching & 2 & 1.4 & 1.4 & 26.7 \\
\hline & 3. Growing & 58 & 39.7 & 39.7 & 66.4 \\
\hline & 4. Transforming & 49 & 33.6 & 33.6 & 100.0 \\
\hline & Total & 146 & 100.0 & 100.0 & \\
\hline
\end{tabular}

Regarding the principal reason of the adoption of the solution SSC (see Table 3.2 below), what stands out immediately is that only $15.8 \%$ of the respondents reported that the SSC is seen mostly as a way of reducing costs. One of the main benefits usually considered in the adoption of the SSCs is the reduction of costs. However, in the respondents' view, this is not the predominant cause. The number may have become notably low, possibly because of about half of the sample (see Figure 3.4 below) are SSCs from the public sector, where historically the improving service quality quest usually comes before the reduction costs item.

Table 3.2 SSC aim

\begin{tabular}{|c|c|c|c|c|c|}
\hline & & Frequency & Percent & Valid Percent & $\begin{array}{c}\text { Cumulative } \\
\text { Percent }\end{array}$ \\
\hline \multirow[t]{6}{*}{ Valid } & Other & 4 & 2.7 & 2.7 & 2.7 \\
\hline & A cut cost alternative & 23 & 15.8 & 15.8 & 18.5 \\
\hline & A new management model & 30 & 20.5 & 20.5 & 39.0 \\
\hline & An organisational solution & 39 & 26.7 & 26.7 & 100.0 \\
\hline & An operational solution & 50 & 34.2 & 34.2 & 73.3 \\
\hline & Total & 146 & 100.0 & 100.0 & \\
\hline
\end{tabular}


The predominance of the public sector (77 or $52.7 \%$ ) compared to the industry (67 or $45.9 \%$ ) between the respondents of the inquiry, presented in Figure 3.4 below, does not cause surprise. This probably occurred because the predominant countries in the sample (see Table 3.3 the following) use the SSCs like government policy for the improvement of the public service, on a great scale.

Figure 3.4 Sector of activity of the SSC

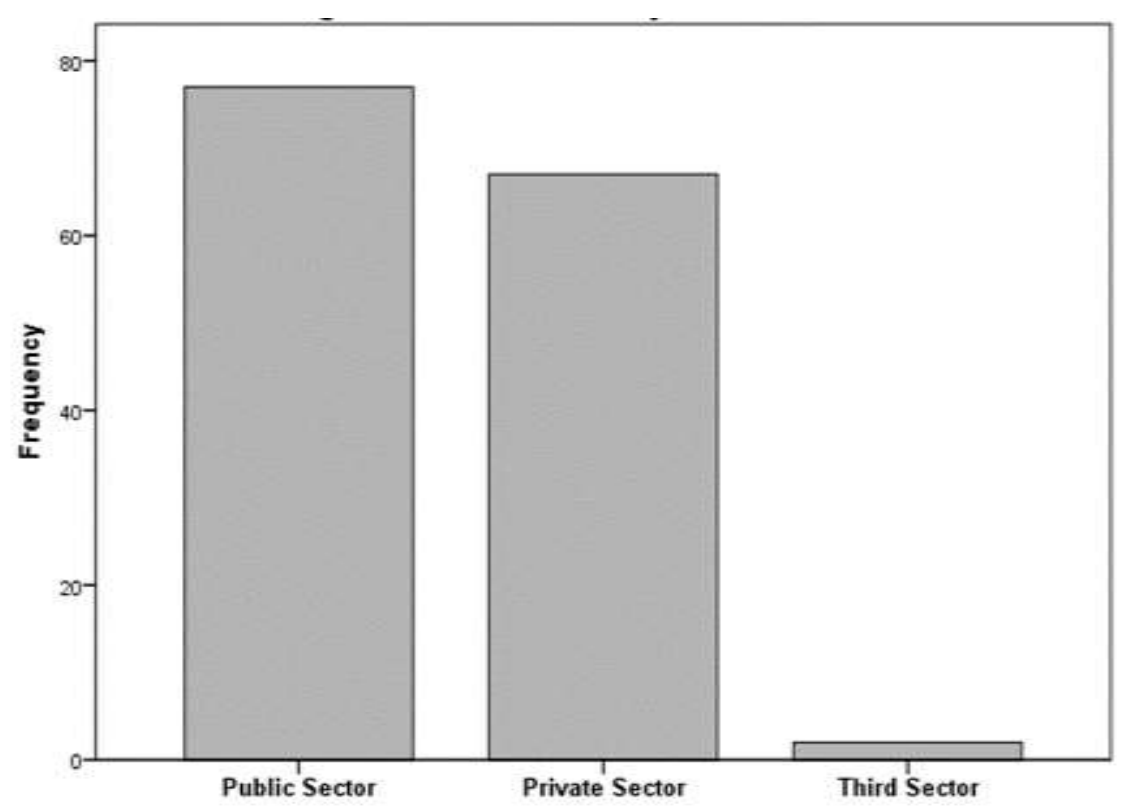

The US and the UK (see Table 3.3 below) represent almost $70 \%$ of the sample (69.9\%). Their governments intensively adopt the SSCs as official solution widely encouraged for public service. This may, to some extent, explain the prevalence of SSCs in the public sector (Figure 3.4 above). Added to this the fact, 20 UK SSCs have been directly invited and accepted to participate in this study. My country, Brazil, is represented by 20 SSC from the private initiative (of an estimated total of about 200 private SSCs - my estimation). This was expected since SSCs from governments hardly exist in the country, with very few exceptions, such as the State Government of Minas Gerais (Thomaz \& Gon, 2013), State Government of Rio Grande do Sul (Krüger, 2012) 
and a few public companies, as Correios (federal courier), Petrobras (oil and gas) and

Sabesp (sanitation and drinking water).

Table 3.3 SSC countries

\begin{tabular}{|c|c|c|c|c|c|}
\hline & & Frequency & Percent & Valid Percent & $\begin{array}{c}\text { Cumulative } \\
\text { Percent }\end{array}$ \\
\hline \multirow[t]{10}{*}{ Valid } & Netherlands & 1 & .7 & .7 & .7 \\
\hline & Portugal & 1 & .7 & .7 & 1.4 \\
\hline & Italy & 2 & 1.4 & 1.4 & 2.8 \\
\hline & Canada & 3 & 2.1 & 2.1 & 4.9 \\
\hline & India & 3 & 2.1 & 2.1 & 7 \\
\hline & Other & 14 & 9.6 & 9.6 & 16.6 \\
\hline & UK & 20 & 13.7 & 13.7 & 30.3 \\
\hline & Brazil & 20 & 13.7 & 13.7 & 44 \\
\hline & USA & 82 & 56 & 56 & 100.0 \\
\hline & Total & 146 & 100.0 & 100.0 & \\
\hline
\end{tabular}

As to the roles of the respondents (see Table 3.4 below), half (73 or $50 \%$ ) consists of managers or general managers.

Table 3.4 Respondent role

\begin{tabular}{|c|c|c|c|c|c|}
\hline & & Frequency & Percent & Valid Percent & $\begin{array}{c}\text { Cumulative } \\
\text { Percent }\end{array}$ \\
\hline \multirow[t]{7}{*}{ Valid } & Other & 24 & 16.4 & 16.4 & 16.4 \\
\hline & Analyst & 16 & 11.0 & 11.0 & 27.4 \\
\hline & Specialist & 19 & 13.0 & 13.0 & 40.4 \\
\hline & Coordinator & 14 & 9.6 & 9.6 & 50.0 \\
\hline & Manager & 51 & 34.9 & 34.9 & 84.9 \\
\hline & General manager & 22 & 15.1 & 15.1 & 100.0 \\
\hline & Total & 146 & 100.0 & 100.0 & \\
\hline
\end{tabular}




\subsubsection{Extrapolation Analysis}

To identify eventual nonresponse error bias (Caruana et al., 1999), an extrapolation analysis was done in accordance with Armstrong \& Overton (1977). This analysis consisted of checking the existence of significant differences between late responders and first responders. The explanation of why this method helps to prevent possible bias of non-representativeness of the sample is that late responders tend to be more alike with non-respondents. That is, if the late respondents are very different from the primary responders, these (the first respondent) probably do not adequately represent the population corresponding to the sample. Demographic data were compared (such as SSC developmental stage, SSC age, and level of experience in SSC) of the one-third $(1 / 3)$ of the respondents of the filled out received questionnaires (ranked in order of arrival) with the two-third (2/3) and the three-third (3/3), totalising 146 cases, and no significant differences were found. This analysis helped to confirm the adequacy and consistency of the data in its entirety, endorsing that the sample is representative of the population of SSCs.

\subsubsection{Exploratory Factor Analysis}

In social sciences' studies, it is common to want to measure something that cannot be measured directly (e.g. constructs, factors, or latent variables). The principle of parsimony present in factor analysis says that a large number of variables observed can be explained by a smaller number of hypothetical variables (latent), non-observed (Agresti \& Agresti, 2009). For example, one may be interested to know if an organisation is market-oriented. As the market orientation cannot be measured directly, as it involves some aspects such as customer orientation and the opening of the company to external influences, it is necessary to resort to the measurement of the aspects that characterise the market orientation. Also, it is interesting to see if the measures taken, measure a 
single variable. The factor analysis allows this to be verified (Field, 2009). Factor analysis is a multivariate statistical method, which allows transforming a large number of variables in a few variables, statistically not correlated, i.e., the factors (Dancey \& Reidy, 2007). It enables to turn a collection of highly correlated predictor variables on one or two factors of approximately the same predictive power (Field, 2009). Factor analysis also helps validate the developed constructs.

There are two usual approaches to factor analysis; confirmatory and exploratory. The confirmatory factor analysis brings together a set of techniques that aim to test and confirm theories or hypotheses concerning the structure of a set of variables (Pallant, 2010). Usually applied in more advanced stages of measuring scale maturity. Exploratory factor analysis helps in understanding the interrelationship of variables sets to refine and simplify constructs, allowing further analysis to validate the conceptual model. Therefore, it is applied at earlier stages of development and confirmation of measurement scales, and so it was chosen for this thesis.

Principal components analysis (PCA) is a technique commonly used in factor analysis, although it is not exactly a factor analysis (FA). For example, while factor analysis uses mathematical models for estimating the factors, PCA transforms the original variables into a reduced set of linear combinations (Pallant, 2010). Conceptually speaking, the PCA generates components, while the FA generates factors. Another difference is that the PCA is based only on the linear correlation of observed variables, and does not distinguish between common variance and the specific variance of the items. When items are retained in a particular component in the PCA, the rates shown include both common variance and specific variance. Many researchers prefer the PCA 
for its simplicity and practicality since the results of both techniques are equivalent. The PCA was also adopted in this thesis.

According to Pallant (2010) some checks and decisions need to take place along three stages of the factor analysis, so that an appropriate factorial structure is obtained: (a) Analysis of the compatibility of the data for factor analysis; (b) Extraction of factors; and (c) Factor rotation and interpretation.

In stage (a) Analysis of the compatibility of the data for factor analysis, it is checked whether the data set is suitable for factor analysis, first, the sample size - which must be greater than 100 cases according to Hair (1998) \& MacCallum et al. (1999). This is a necessary step because the correlation coefficients in very small samples are less reliable - According to this criterion, it was found that the research sample for this thesis is adequate, with its 146 respondents. Next, it is verified the strength of the relationship between the variables (issues, or items). It is necessary to inspect the correlation matrix in search of coefficients greater than $.3(\mathrm{r}<.30)$, since if there are few correlations above this level the factor analysis can be compromised. As main criteria for dropping items, I considered loadings lower than .50. Besides these, it is necessary to check two other statistical measures to confirm the factorability of data. Bartlett's sphericity test, where it must have $\mathrm{p}<.05$ to be significant, and the measure of the adequacy of samples of Kaiser-Meyer-Olkin (KMO), ranging between 0 and 1, with .6 being considered the minimum value for a proper factor analysis (Pallant, 2010). The results of these analyses are discussed further down.

In stage (b) Extraction of factors, it seeks to find the lowest number of factors that represents the interrelationship of the variables in the best possible way. The techniques applied here were the principal components analysis (PCA), mentioned above, with 
eigenvalue (Kaiser's criterion) above 1; scree plot analysis (verifying where the curve becomes horizontal), and parallel analysis, using the Monte Carlo PCA program as a comparative basis, to ratify the components initially indicated.

Finally, in stage (c) Factor rotation and interpretation, after the minimum number of factors was established, it seeks to interpret them and to do that, it makes the rotation of the factors which highlights the higher and lower correlations. Thus, the loadings are evidenced getting easier to interpret. In this work, I performed factor rotation applying the orthogonal method Varimax. To assess the reliability of the factorial structure, although it could have adopted other methods, such as the greatest lower-bound or intraclass correlation coefficient (Sijtsma, 2009; Narver et al., 2004), I used the Cronbach's alpha, since it attends the necessity of my study and is the most widely used method for this purpose. This internal consistency index assesses the degree to which the items in an array of data are correlated linearly with each other. I considered the following scale of adequacy of Cronbach's alpha values, in accordance with George \& Mallery (2003): $\mathrm{a}>0.90$ excellent; $\mathrm{a}>0.80$ good; $\mathrm{a}>0.70$ acceptable; $\mathrm{a}>0.60$ questionable; $\mathrm{a}>0.50$ poor; $\mathrm{a}<0.50$ unacceptable.

Factor analyses carried out for each conceptual model construct designed for this thesis - The questionnaire subscales (Market Orientation, Culture, Leadership, Resources, Readiness for Change, Excellence, and Performance) were subjected to principal components analysis (PCA) in SPSS version 22, using the file with 146 responses. Before carrying out the analysis, a checking of data suitability aiming for factor analysis was driven, and it presented the results below. All, or the most, variables of the correlation matrix presented various correlation coefficients greater than 0.3 . The Kaiser-Meyer-Olkin (KMO) test reached from 0.83 - which is classified as middling to 
meritorious according to Kaiser (1974) — to 0.92 (marvellous according to that classification), attesting that the variables are correlated. The Bartlett sphericity was statistically significant $(\mathrm{p}<.0005)$, representing factorisable data in the correlation matrix. The following I show, using Market Orientation as an example, I carried out for each factor, starting with the descriptive statistics of in Table 3.5. All the analyses were double-checked, by a statistic expert, and my first supervisor, experienced in quantitative research.

Table 3.5 Descriptive statistics of Market Orientation

\begin{tabular}{cccc}
\hline Item & Mean & Std. Deviation & Analysis N \\
\hline M01 & 5.32 & 1.644 & 146 \\
M02 & 5.55 & 1.395 & 146 \\
M03 & 5.28 & 1.570 & 146 \\
M04 & 5.58 & 1.338 & 146 \\
M05 & 5.38 & 1.458 & 146 \\
M06 & 5.11 & 1.674 & 146 \\
M07 & 5.18 & 1.583 & 146 \\
\hline
\end{tabular}

The PCA revealed initially two components with eigenvalue above 1 , corresponding, respectively, to $51.13 \%$ and $15.44 \%$ of the total variance. An analysis of the scree plot confirmed the initial identification of two components to be maintained (Field, 2009). Similarly, a parallel analysis using Monte Carlo PCA program (Pallant, 2010) confirmed the retention of two components, since exceeded the randomly generated values in the data matrix of the same size ( 7 variables $x 146$ respondents). Thus, it was decided to maintain two components.

Once kept the two components, which explain $66.57 \%$ of the total variance, an orthogonal Varimax rotation was performed to support the interpretability. The result of the rotation fits into the concept of 'simple structure' of Thurstone (Child, 2006) with, 
for example, each factor having at least one high loading and the other, very low. No items were dropped because there was no loading smaller than .50 , the main criteria adopted for dropping variables. As shown in Table 3.6 below, the interpretation of data is consistent in both proposed components. Also, two subscales of Market Orientation could be established here (for example, the Component 1 related to the client and Component 2 related to monitoring), but were not, as the construction and/or refinement of the scale are not in the scope of this thesis. The two components showed a good degree of internal consistency with acceptable Cronbach's alpha of .791 and .758 respectively (George \& Mallery, 2003; Bagozzi \& Yi, 1988). Component 2 would have good Cronbach's alpha of .819 (George \& Mallery, 2003) if the M05 item were dropped, but considering the results of other analyses, I decided to keep it.

Table 3.6 Exploratory factor analysis results - Rotated Component Matrix - Market Orientation

\begin{tabular}{|c|c|c|}
\hline \multirow{2}{*}{ Item } & \multicolumn{2}{|c|}{ Factor loadings } \\
\hline & Component 1 & Component 2 \\
\hline $\begin{array}{l}\text { M01 - Our business objectives are driven } \\
\text { primarily by customer/citizen satisfaction }\end{array}$ & .826 & \\
\hline $\begin{array}{l}\text { MO2 - We constantly monitor our level of } \\
\text { commitment and orientation to serving } \\
\text { customer/citizen needs }\end{array}$ & .826 & \\
\hline $\begin{array}{l}\text { MO3 - We measure customer/citizen } \\
\text { satisfaction systematically and frequently }\end{array}$ & .610 & \\
\hline $\begin{array}{l}\text { M04 - We have routine or regular measures of } \\
\text { customer/citizen service }\end{array}$ & & .684 \\
\hline $\begin{array}{l}\text { MO5 - I believe this SSC exists primarily to serve } \\
\text { customers/citizens }\end{array}$ & .669 & \\
\hline $\begin{array}{l}\text { M06 - We poll end-users at least once a year to } \\
\text { assess the quality of our products and services }\end{array}$ & & .871 \\
\hline $\begin{array}{l}\text { MO7 - Data on customer satisfaction are } \\
\text { disseminated at all levels in this business unit on } \\
\text { a regular basis }\end{array}$ & & .788 \\
\hline Eigenvalue & 3.579 & 1.080 \\
\hline$\%$ of variance explained & 51.13 & 15.44 \\
\hline Cronbach alpha & .791 & .758 \\
\hline
\end{tabular}




\subsubsection{Correlation}

I used the correlation to start the validation of the conceptual model proposed by this thesis. The correlation is a statistical method used to investigate (using a correlation coefficient) the association between two continuous variables, which may be a dependent variable and an independent variable or two independent variables (Dancey \& Reidy, 2007). According to Nunnally \& Bernstein (1994), the correlation demonstrates the degree of relationship between two variables. In this work the Pearson ProductMoment correlation coefficient was used (r), which varies between-1 and 1, e establishes the direction and strength of the linear existent association between the variables. On the positive relationship between two variables when one increases the other also increases. In the negative relation, when one lessens the other one increases (Field, 2009). While the sign of the coefficient of correlation shows the direction of the association, its absolute magnitude represents the strength of the association. For example, a coefficient of Pearson $r=0.950$ corresponds to a positive and strong association between two variables, while a coefficient $r=-.250$ represents a negative and weak association. The value zero indicates the non-existence of relationship, and the coefficient 1 expresses a perfect relation or, in other words, a variable can be determined when the value of the other one is known. For a correct use of the correlation, it is important to check, for example, if there are no outliers and if the variables present linear relationship. The latest can be identified graphically with a scatterplot, so it allows to visualize if the relation of two variables corresponds or not to a straight line.

The following are the procedures and results of the correlation analysis carried out for the constructs of the conceptual model developed and tested in this study, and discussed in Section 2.6 Conceptual Model. 
Procedures - The variables used in the analyses of correlation discussed below were those generated in Exploratory Factor Analysis (EFA) and included as new columns in the data file (FAC1_1, for example) by SPSS for each construct, as discussed in the previous section. Each of these corresponds to the new SPSS generated component during the Principal Components Analysis (PCA). In the case of the constructs Culture and Market Orientation, SPSS generated instead of one, two new components during the PCA. In this case, Component 1 , accounting for the greatest loads, was used in the correlation analysis. Before the correlation analysis of each pair of independent and dependent variables, the corresponding scatter plots were examined to confirm the linearity prerequisite of the relationship, and the existence of outliers was examined. These tests confirmed the appropriateness of all relations for correlation analysis, with positive linearity; outliers were not identified.

Results - following are presented the results and discussions of the driven analyses conducted using Pearson Product-Moment correlation coefficient (r). To categorize the strength of the association of the variables it was considered the scale of Cohen suggested by Pallant (2010): Small, r = .10 to .29; Medium, r = .30 to .49; and Large, $r=$ .50 to 1.0 .

The first analysis (see Table 3.19 below) investigated the correlation of each of the independent variables, Culture, Leadership, Resources and Readiness for Change, with the dependent variable Excellence. It was found to be strong (Large) positive correlation between each of these independent variables and the dependent variable analysed, with r ranging from .73 to .81 . 
Table 3.19 Correlation Analysis of independent variables Culture, Leadership, Resources and Readiness for Change, and the dependent variable Excellence

\begin{tabular}{lccccc}
\hline & Culture & Leadership & Resources & $\begin{array}{c}\text { Readiness } \\
\text { for Change }\end{array}$ & Excellence \\
\hline Culture & 1 & $.79^{* *}$ & $.74^{* *}$ & $.68^{* *}$ & $.73^{* *}$ \\
Leadership & $.79^{* *}$ & 1 & $.73^{* *}$ & $.77^{* *}$ & $.75^{* *}$ \\
Resources & $.74^{* *}$ & $.73^{* *}$ & 1 & $.79^{* *}$ & $.81^{* *}$ \\
$\begin{array}{l}\text { Readiness } \\
\text { for Change }\end{array}$ & $.68^{* *}$ & $.77^{* *}$ & $.79^{* *}$ & 1 & $.80^{* *}$ \\
Excellence & $.73^{* *}$ & $.75^{* *}$ & $.81^{* *}$ & $.80^{* *}$ & 1 \\
\hline
\end{tabular}

**. Correlation is significant at the 0.01 level (2-tailed). $\mathrm{N}=146$. Pearson correlation $(\mathrm{r})$.

The second analysis investigated the correlation between the variables Excellence and Market Orientation. It was found a positive correlation ('low' Medium) between these variables analysed, with $r=.36$.

The third analysis investigated the correlation between the variables Market Orientation and Performance. It was found a positive correlation ('low' Medium) between these variables analysed, also with $r=.36$.

The fourth analysis investigated the correlation between the variables Performance and Excellence. It was found a positive correlation (Large) between these variables analysed, with $r=.86$

The correlation analyses performed ratified the first level of validation of the conceptual model, demonstrating that the associations of the constructs identified during the literature review were corroborated empirically. However, the Pearson Product-Moment correlation coefficients showed that the degree of correlation between Excellence and Performance $(r=.86)$ is much more expressive than between Market Orientation and Performance $(r=.36)$, while the variable Market Orientation showed correlation, to a certain extent, shy $(r=.36)$ with the variable Excellence. The direct 
relation between Excellence and Performance was not initially identified by the proposed model. These findings suggest that while the impact of Excellence in Market Orientation is only medium, the impact of Excellence in the Performance of the SSCs is surprisingly higher. But, would be this finding true for both SSCs from the public sector and industry? The answer is yes, with Excellence impacting Performance almost equally in both sectors ( .84 in the public sector and .88 in the industry), but with an important difference. The correlation between Market Orientation and Performance is much stronger - almost double - in the industry that has $r=.45(\mathrm{~N}=77)$ against $r=.23$ in the public sector $(\mathrm{N}=67)$.

\subsubsection{Multiple Regression}

The analysis of correlation seen above is very useful to understand the relationship between variables, but it is not conclusive as to the validation of the conceptual model because it does not provide information about the predictive power of the variables. To test the hypotheses and finish the validation of the conceptual model, I used multiple regression analysis. Multiple regression is a set of techniques used to explore and explain the relationship between independent variables and the dependent variable, as an extension of the correlation analysis (Agresti \& Agresti, 2009). These techniques allow more sophisticated investigations between variables as, for example, if a set of variables can predict an outcome. It is also possible through multiple regression to identify which variable in a set of variables is the best predictor (Field, 2009). Therefore, multiple regression is an appropriate technique to complete the validation of the conceptual model and hypotheses of this thesis with more in-depth analysis.

The following are the procedures and results of multiple regression analyses conducted for the constructs of the proposed conceptual model. Also, further down, in 
Section 3.11.6 Hypotheses Validation are presented the results of the validation of each of the six formulated hypotheses.

Procedures - I used the same variables (factors) applied to the correlation analysis (extracted by SPSS), ensuring before the assumptions for an effective test were met. These assumptions are, independence of errors (residuals); a linear relationship between predictors variables and the outcome variable; residuals homoscedasticity; absence of multicollinearity; and no significant outliers (Field, 2009; Pallant, 2010). After analysing the applicability of each of the main regression methods - Standard Multiple Regression, Hierarchical Multiple Regression, and Stepwise Multiple Regression -, I selected the Standard Multiple Regression method as the most suitable for the validation of the conceptual model and the hypotheses. This method is typically used when it is necessary to understand how much variance can be explained by a set of predictor variables and by each variable individually (Pallant, 2010). Predictors are inserted simultaneously into the equation, and each independent variable is analysed to know its predictive power. One of the limitations of the Standard Multiple Regression is that it does not make possible to control straightly the shared variance between the independent variables, which may lead to conclusion errors (Hair, 1998; Field, 2009). This limitation was avoided in this research using the components (factors) generated in the Principal Components Analysis during the factor analysis reported earlier in this chapter.

According to Field (2009) and Pallant (2010), so that the results of the application of this method are technically valid, six assumptions must be met, under penalty of getting distorted results. (1) Sample size, because only a representative sample of the population allows the generalisation. The sample size of this research $(\mathrm{N}=146)$ is above 
the recommended by Hair (1998) and MacCallum et al. (1999) of, at least, 100 cases; (2) linearity, i.e. independent variables must relate linearly with the dependent variable, individually and collectively; (3) homoscedasticity, because in each level of the predictors variables, the variance of the residual term must be constant. The residuals at each level of the predictors must have the same variance; when the variances are not equal exists heteroscedasticity (4) multicollinearity, which refers to the relationship between the independent variables, and exists when these are highly correlated, with $\mathrm{r}$ $=.9$ or above. I used the Variance Inflation Factor indicator (VIF) seeking for values above 10, which could be problematic, indicating multicollinearity; (5) unusual points, because the multiple regression is quite sensitive to very high or very low scores (standardised residual values less than -3.3 or above 3.3 ) and this verification must include both independent and dependent variables; and (6) residuals normality, since it is assumed that the residuals are distributed within a normal curve, with zero mean. All these checks were conducted and approved before the regression analyses were applied. Results - The following are the results of the three multiple regression analyses carried out.

First analysis: A multiple regression was run to predict Excellence from Culture, Leadership, Resources, and Readiness for Change. The assumptions of sample size, linearity, homoscedasticity, multicollinearity, unusual points and residuals normality were met. These variables, statistically significant, predicted Excellence, $\mathrm{F}(4,141)=$ 105.633, $\mathrm{p}<.0005$, adj. $\mathrm{R} 2=.74$. All variables, individually, added statistically significantly to the prediction, $\mathrm{p}<.05$, except Leadership with the sig. $=.13$. Regression coefficients and standard errors can be found in Table 3.20 below. 
Table 3.20 Summary of the first Multiple Regression Analysis

\begin{tabular}{lccc}
\hline Variable & B & Std. Error & Beta \\
\hline (constant) & .000 & .042 & .153 \\
Culture & .153 & .075 & .125 \\
Leadership & .125 & .082 & .373 \\
Resources & .373 & .077 & .301 \\
$\begin{array}{l}\text { Readiness } \\
\text { for Change }\end{array}$ & .301 & .078 & \\
\hline
\end{tabular}

Note: $\mathrm{p}<.05 ; \mathrm{B}=$ Unstandardised Coefficients; Std. Error = Standard error of the coefficient; Beta $=$ standardised coefficient

Second analysis: A multiple regression was run to predict Market Orientation from Excellence. The assumptions of sample size, linearity, homoscedasticity, multicollinearity, unusual points and residuals normality were met. The variable statistically significantly predicted Market Orientation, $F(1,144)=21.067, \mathrm{p}<.0005$, adj. $\mathrm{R} 2=.12$. Regression coefficients and standard errors can be found in Table 3.21 below.

Table 3.21 Summary of the first Multiple Regression Analysis

\begin{tabular}{lccc}
\hline Variable & B & Std. Error & Beta \\
\hline (constant) & .000 & .078 & \\
Excellence & .357 & .078 & .357 \\
\hline
\end{tabular}

Note: $\mathrm{p}<.05 ; \mathrm{B}=$ Unstandardised Coefficients; Std. Error = Standard error of the coefficient; Beta $=$ standardised coefficient

Third analysis: A multiple regression was run to predict Performance from Market Orientation. The assumptions of sample size, linearity, homoscedasticity, multicollinearity, unusual points and residuals normality were met. The variable statistically significantly predicted Market Orientation, $F(1,144)=21.832$, p < .0005, 
adj. $\mathrm{R} 2=.13$. Regression coefficients and standard errors can be found in Table 3.22 below.

Table 3.22 Summary of the second Multiple Regression Analysis

\begin{tabular}{lccc}
\hline Variable & B & Std. Error & Beta \\
\hline (constant) & .000 & .077 & \\
Market Orientation & .363 & .078 & .363 \\
\hline
\end{tabular}

Note: $\mathrm{p}<.05 ; \mathrm{B}=$ Unstandardised Coefficients; Std. Error = Standard error of the coefficient;

Beta $=$ standardised coefficient

\subsubsection{Hypotheses Validation}

This section discusses the results of the analysis conducted on acceptance or rejection of hypotheses formulated for this study. Figure 3.5 below illustrates the conceptual model and the six hypotheses tested, formulated with an expected positive relation between the corresponding variables. The hypotheses presented and initially discussed in Section 2.6.4 are H1: Culture relates positively to excellence; H2: Leadership relates positively to excellence; H3: Resources relates positively to excellence; H4: The greater the readiness for change, the higher the level of the excellence; H5: excellence relates positively to market orientation; H6: Market orientation relates positively to performance. 
Figure 3.5 Conceptual model of factors influencing SSC performance, with the hypotheses

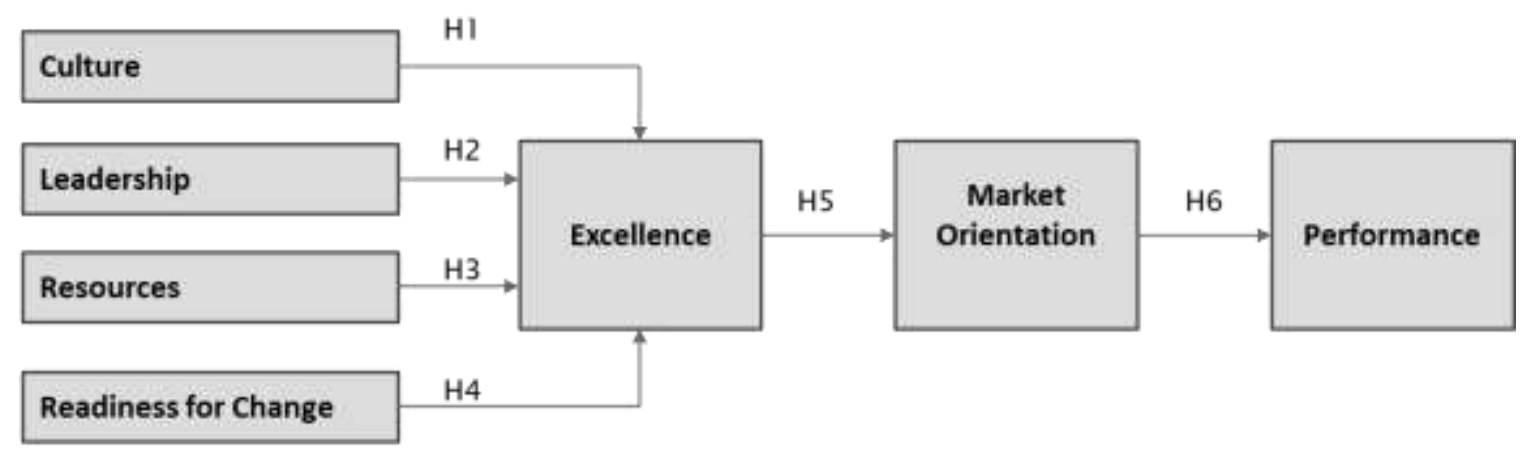

As summarised in Table 3.23 below, five of the six hypotheses (H1 and H3 to H6) have been validated (supported), with the respective null hypotheses rejected. The null hypothesis says, by definition, that there is no relationship between the variables (Creswell, 2013). The hypothesis H2 was rejected (fail to reject the null hypothesis) since the observed result cannot be considered statistically significant. That is, in this survey the predictor variable Leadership seems not to contribute significantly to estimate Excellence; although Leadership correlation is significant with all other variables, as seen in Section 3.11.4. The t-test, whose result is shown in that table, consists in formulating a null hypothesis and, consequently, an alternative hypothesis, calculate the value of $\mathrm{t}$ and draw conclusions as to the prediction power of the variable analysed, i.e., knowing the significance of the t-test of each predictor. 
Table 3.23 Summary of the validation of the hypotheses

\begin{tabular}{|c|c|c|c|c|}
\hline Hypotheses & Sign & Std. Coef. & t-value & $\begin{array}{l}\text { Hypothesis } \\
\text { test }\end{array}$ \\
\hline $\begin{array}{l}\text { H1: Culture relates positively to PSSC } \\
\text { excellence }\end{array}$ & + & .153 & $2.037^{* *}$ & Supported \\
\hline $\begin{array}{l}\text { H2: Leadership relates positively to PSSC } \\
\text { excellence }\end{array}$ & + & .125 & $1.527^{*}$ & Rejected \\
\hline $\begin{array}{l}\text { H3: Resources relates positively to PSSC } \\
\text { excellence }\end{array}$ & + & .373 & $4.843^{* *}$ & Supported \\
\hline $\begin{array}{l}\text { H4: The greater the readiness for change, } \\
\text { the higher the level of the PSSC excellence }\end{array}$ & + & .301 & $3.874^{* *}$ & Supported \\
\hline $\begin{array}{l}\text { H5: PSSC excellence relates positively to } \\
\text { market orientation }\end{array}$ & + & .357 & $4.590^{* *}$ & Supported \\
\hline $\begin{array}{l}\text { H6: Market orientation relates positively to } \\
\text { PSSC performance }\end{array}$ & + & .363 & $4.672^{* *}$ & Supported \\
\hline
\end{tabular}

Note: ${ }^{*} \mathrm{p}=.13 ;{ }^{* *} \mathrm{p}<.05$

\subsubsection{Common Method Bias Evaluation}

Since all participants responded to both the questions of the independent variables and dependent variables, the variance arising from the use of the same method (common method variance) could decrease or increase the estimated relationships. To verify the existence of common method bias, Harman's one-factor test was applied (Podsakoff \& Organ, 1986; Theodosiou \& Katsikea, 2013). If there were common method bias, the Principal Component Analysis employment would produce a single factor from the submitted data; and this single factor would explain most of the variance. The test was performed with the use of the unrotated solution, and a general factor did not appear. The first factor explained $38 \%$ of the variance, so below $50 \%$ and confirming the absence of common method bias (see Table 3.24 below, with the first 17 out of 71 components). 
Table 3.24 PCA - Total Variance Explained - Harman's Test

\begin{tabular}{|c|c|c|c|c|c|c|}
\hline \multirow[b]{2}{*}{ Component } & \multicolumn{3}{|c|}{ Initial Eigenvalues } & \multicolumn{3}{|c|}{$\begin{array}{l}\text { Extraction Sums of Squared } \\
\text { Loadings }\end{array}$} \\
\hline & Total & $\begin{array}{c}\% \text { of } \\
\text { Variance }\end{array}$ & $\begin{array}{c}\text { Cumulative } \\
\%\end{array}$ & Total & $\begin{array}{c}\% \text { of } \\
\text { Variance }\end{array}$ & $\begin{array}{c}\text { Cumulative } \\
\%\end{array}$ \\
\hline 1 & 26.856 & 37.826 & 37.826 & 26.856 & 37.826 & 37.826 \\
\hline 2 & 3.505 & 4.936 & 42.762 & & & \\
\hline 3 & 3.010 & 4.239 & 47.001 & & & \\
\hline 4 & 2.268 & 3.194 & 50.195 & & & \\
\hline 5 & 1.922 & 2.707 & 52.902 & & & \\
\hline 6 & 1.805 & 2.542 & 55.444 & & & \\
\hline 7 & 1.711 & 2.410 & 57.853 & & & \\
\hline 8 & 1.487 & 2.094 & 59.947 & & & \\
\hline 9 & 1.449 & 2.041 & 61.989 & & & \\
\hline 10 & 1.403 & 1.976 & 63.965 & & & \\
\hline 11 & 1.284 & 1.808 & 65.773 & & & \\
\hline 12 & 1.185 & 1.669 & 67.442 & & & \\
\hline 13 & 1.080 & 1.521 & 68.963 & & & \\
\hline 14 & 1.045 & 1.472 & 70.435 & & & \\
\hline 15 & 1.011 & 1.424 & 71.859 & & & \\
\hline 16 & .968 & 1.364 & 73.222 & & & \\
\hline 17 & .876 & 1.235 & 74.457 & & & \\
\hline
\end{tabular}

\subsubsection{Independent Samples T-test}

The Independent samples t-test is used to compare means between two groups and check if there are significant differences. I applied the test to compare the SSCs of the public sector (see 1 in the Table 3.25 below) and those in the industry (see 2 in the Table 3.25 below), analysing all variables of the conceptual model (market orientation, culture, leadership, resources, readiness for change, excellence, and performance). The results show no significant differences and are presented below. Table 3.25 below shows the descriptive statistics of the two groups, with the means and standard deviations, showing that do not exist important differences in the comparison of each variable to SSCs of the two sectors. Table 3.26 shows the specific results of the t-test. 
Table 3.25 Group Statistics for Each Conceptual Model Variable

\begin{tabular}{|ll|r|r|r|r|}
\hline & SECTOR & N & Mean & Std. Deviation & \multicolumn{1}{c|}{$\begin{array}{c}\text { Std. Error } \\
\text { Mean }\end{array}$} \\
\hline TOTAL_MO & 1 & 77 & 36,7143 & 8,25555 &, 94081 \\
& 2 & 67 & 38,0896 & 6,77502 &, 82770 \\
\hline TOTAL_CULT & 1 & 77 & 50,8182 & 9,29591 & 1,05937 \\
& 2 & 67 & 50,4328 & 8,11886 &, 99188 \\
\hline TOTAL_LEAD & 1 & 77 & 42,4416 & 10,24502 & 1,16753 \\
& 2 & 67 & 43,7015 & 7,99055 &, 97620 \\
\hline TOTAL_RESOUR & 1 & 77 & 61,0519 & 13,22965 & 1,50766 \\
& 2 & 67 & 63,4776 & 10,77646 & 1,31655 \\
\hline TOTAL_READIN & 1 & 77 & 48,4675 & 10,06503 & 1,14702 \\
& 2 & 67 & 50,2239 & 8,80396 & 1,07557 \\
\hline TOTAL_EXCELL & 1 & 77 & 71,7403 & 15,16610 & 1,72834 \\
& 2 & 67 & 73,8358 & 14,45536 & 1,76600 \\
\hline TOTAL_PERFOR & 1 & 77 & 52,8442 & 10,39745 & 1,18490 \\
& 2 & 67 & 53,0299 & 9,91627 & 1,21147 \\
\hline
\end{tabular}

$1=$ Public Sector and 2 = Private Sector

As shown in the table 3.26 below, the variables analysed by comparing the public and private SSCs do not show statistically significant differences when looking to the Sig (2-tailed) measures; the Sig value of Levene's test is greater than .05, except for the variable Leadership, but whose Sig. (2-tailed), which aims to test for equality of means, is appropriate. 
Table 3.26 Independent Samples Test (public versus private SSC)

\begin{tabular}{|c|c|c|c|c|c|c|c|c|c|c|}
\hline & & \multicolumn{2}{|c|}{$\begin{array}{l}\text { Levene's Test for } \\
\text { Equality of } \\
\text { Variances }\end{array}$} & \multicolumn{7}{|c|}{ t-test for Equalty of Means } \\
\hline & & \multirow[b]{2}{*}{$\mathrm{F}$} & \multirow[b]{2}{*}{ Sig. } & \multirow[b]{2}{*}{$\mathrm{t}$} & \multirow[b]{2}{*}{ if } & \multirow{2}{*}{$\begin{array}{l}\text { Sig. (2- } \\
\text { tailed) }\end{array}$} & \multirow{2}{*}{$\begin{array}{c}\text { Mean } \\
\text { Difference }\end{array}$} & \multirow{2}{*}{$\begin{array}{l}\text { Std. Error } \\
\text { Difference }\end{array}$} & \multicolumn{2}{|c|}{$\begin{array}{c}85 \% \text { Confidence interval of } \\
\text { the Difference }\end{array}$} \\
\hline & & & & & & & & & Lower & Upper \\
\hline \multirow[t]{2}{*}{ TOTAL_MO } & $\begin{array}{l}\text { Equal variances } \\
\text { assumed }\end{array}$ & 2,305 &, 131 & $-1,089$ & 142 & .281 & $-1,37527$ & 1,27029 & $-3,88640$ & 1,13586 \\
\hline & $\begin{array}{l}\text { Equal variances } \\
\text { not assumed }\end{array}$ & & & $-1,099$ & 141,54 & .274 & $-1,37527$ & 1,25309 & $-3,85244$ & 1,10190 \\
\hline \multirow[t]{2}{*}{ TOTAL_CULT } & $\begin{array}{l}\text { Equal variances } \\
\text { assumed }\end{array}$ & 472 &, 493 & .263 & 142 & .793 & 39535 & 1,46495 & $-2,51059$ & 3,28128 \\
\hline & $\begin{array}{l}\text { Equal variances } \\
\text { not assumed }\end{array}$ & & &, 260 & 142,00 &, 791 &, 30536 & 1,45123 & -2.48347 & 3,25416 \\
\hline \multirow[t]{2}{*}{ TOTAL_LEAD } & $\begin{array}{l}\text { Equal variances } \\
\text { assumed }\end{array}$ & 4,457 &, 037 & -814 & 142 & .417 & $-1,25993$ & 1,54801 & $-4,32006$ & 1,80019 \\
\hline & $\begin{array}{l}\text { Equal variances } \\
\text { not assumed }\end{array}$ & & & -828 & 140,39 & .409 & $-1,25993$ & 1,52187 & $-4,26868$ & 1,74881 \\
\hline \multirow[t]{2}{*}{$\begin{array}{l}\text { TOTAL RES } \\
\text { OUR }\end{array}$} & $\begin{array}{l}\text { Equal variances } \\
\text { assumed }\end{array}$ & 2,561 & .112 & $-1,195$ & 142 & .234 & $-2,42566$ & 2,03010 & $-6,43879$ & 1,58746 \\
\hline & $\begin{array}{l}\text { Equal vanances } \\
\text { not assumed }\end{array}$ & & & $-1,212$ & 141.41 & .228 & 2,42566 & 2,00159 & -6.38256 & 1,53124 \\
\hline \multirow[t]{2}{*}{$\begin{array}{l}\text { TOTAL_READ } \\
\text { IN }\end{array}$} & $\begin{array}{l}\text { Equal vaniances } \\
\text { assumed }\end{array}$ & .444 & .506 & $-1,107$ & 142 & .270 & $-1,75635$ & 1,58712 & -4.89379 & 1,38109 \\
\hline & $\begin{array}{l}\text { Equal variances } \\
\text { not assumed }\end{array}$ & & & $-1,117$ & 141.99 & .266 & $-1,75635$ & 1,57242 & $-4,86473$ & 1,35203 \\
\hline \multirow[t]{2}{*}{$\begin{array}{l}\text { TOTAL_EXCE } \\
\amalg\end{array}$} & $\begin{array}{l}\text { Equal variances } \\
\text { assumed }\end{array}$ & 185 & .668 &,- 845 & 142 & .399 & $-2,09556$ & 2,47932 & -6.99670 & 2,80558 \\
\hline & $\begin{array}{l}\text { Equal variances } \\
\text { not assumed }\end{array}$ & & &,- 848 & 140,80 &, 398 & $-2,03556$ & 2,47102 & $-6,98065$ & 2,78953 \\
\hline \multirow[t]{2}{*}{$\begin{array}{l}\text { TOTAL_PERF } \\
\text { OR }\end{array}$} & $\begin{array}{l}\text { Equal variances } \\
\text { assumed }\end{array}$ & 315 &, 575 &,- 108 & 142 & 913 &,- 18569 & 1,70021 & $-3,54669$ & 3,17530 \\
\hline & $\begin{array}{l}\text { Equal variances } \\
\text { not assumed }\end{array}$ & & & -110 & 140,79 &, 913 &,- 18569 & 1,69459 & $-3,535 \mathrm{~B} 3$ & 3,16444 \\
\hline
\end{tabular}

Therefore, the results suggest that there are no practical differences between the groups of SSC public and private.

In this section, there were presented analyses employed to the data of the survey carried out with the sample of 146 SSCs, with the objective to validate the conceptual model and the respective hypotheses of research. The factors (components) representing each construct were extracted through Exploratory Factor Analysis. The most significant factor for each construct was subjected to correlation analysis, which confirmed a positive correlation between the variables of the conceptual model, as originally expected, with Medium or Large strength of association. Emphasis on the discovery of that the degree of correlation between Excellence and Performance is much more expressive $(r=.86)$ than the degree of correlation between Market Orientation and Performance $(\mathrm{r}=.36)$, differently of the previously imagined, while the variable Market 
Orientation was correlated to a certain timid point $(r=.36)$ with variable Excellence, which was also not envisioned. As for the multiple regression analyses, they confirmed the causal relationships of the model, except for the variable Leadership, which did not provide sufficient significance, and led the hypothesis $\mathrm{H} 2$ to fail to reject the null hypothesis. An independent samples t-test was also carried out confirming the similarities of the SSCs from the industry and the public sector regarding the conceptual model.

The next section, Qualitative Action Research, discusses the methods and the results of the Action Learning Set part of the research, developed with the goal of transforming the findings and conclusions of the quantitative and exploratory qualitative research into workable recommendations, which can be implemented by the SSCs, especially the ones from the public sector.

\subsection{Qualitative Action Research}

During the literature review were identified differences, of both form and content, between public management and private management. Some examples of these differences include lower commitment of civil servants; the tendency of public officials to favour the politicians who sponsor and manage their funding; the existence of multiple sources of potentially conflicting authority; the discrepancy between the objectives of public service and the outcomes delivered; concern with rules and not results; less freedom of public officials to respond to the circumstances; and the characteristic of the public sector to be more concerned with inputs and process (insideout view), while the industry is more concerned with outputs and outcomes (tending to an outside-in view). To understand the extent to which these or other differences are part of the context of SSCs of the public and private sectors, two open-ended questions 
were elaborated and submitted to the respondents during the survey: (1) "Do you believe there are significant differences between private sector and public-sector SSC models? If yes, what are the main differences? (2) If not, why not?" The responses were collected and analysed using content analysis techniques, as explained below.

\subsubsection{Analysis of Content}

To analyse the answers to the open-ended questions, I applied techniques of analysis of content, which are a structured way to organize, describe and interpret the content of texts and surveys (Bardin, 1977; Creswell, 2007). The content analysis was performed using qualitative data analysis methodology in three stages, adapted from the work of Bardin (1977) and Creswell (2007). The first stage, called 'pre-analysis,' basically consisted of a preliminary reading of the responses, to have the general knowledge necessary for categorisation to be held in the next stage. The second stage, 'Exploration' of the material, consisted of more detailed analysis with excerpts of text and categorisations seeking to extract, group and describe the concepts (codes) existing in the analysed texts. Finally, the third stage, 'Interpretation', included the explanation of the original content declared or latent in the material analysed. Creating categories in this stage take into account the five principles of Bardin (1977): mutual exclusion, between categories; homogeneity, inside the categories; relevance, or not distortion, of the transmitted message; fertility for inferences; and objectivity, or understanding and clarity. To support this content analysis, it was used the software SPSS Text Analytics for Surveys version 4.0 (see Figure 3.6 below), which applies natural language processing and streamlines the extraction and categorisation of concepts from the examined texts. The following is a brief description of the found frequencies and then goes to the description of the categorisation and presentation of results. 
Figure 3.6 Screen snapshot of the software SPSS Text Analytics for Surveys version 4.0

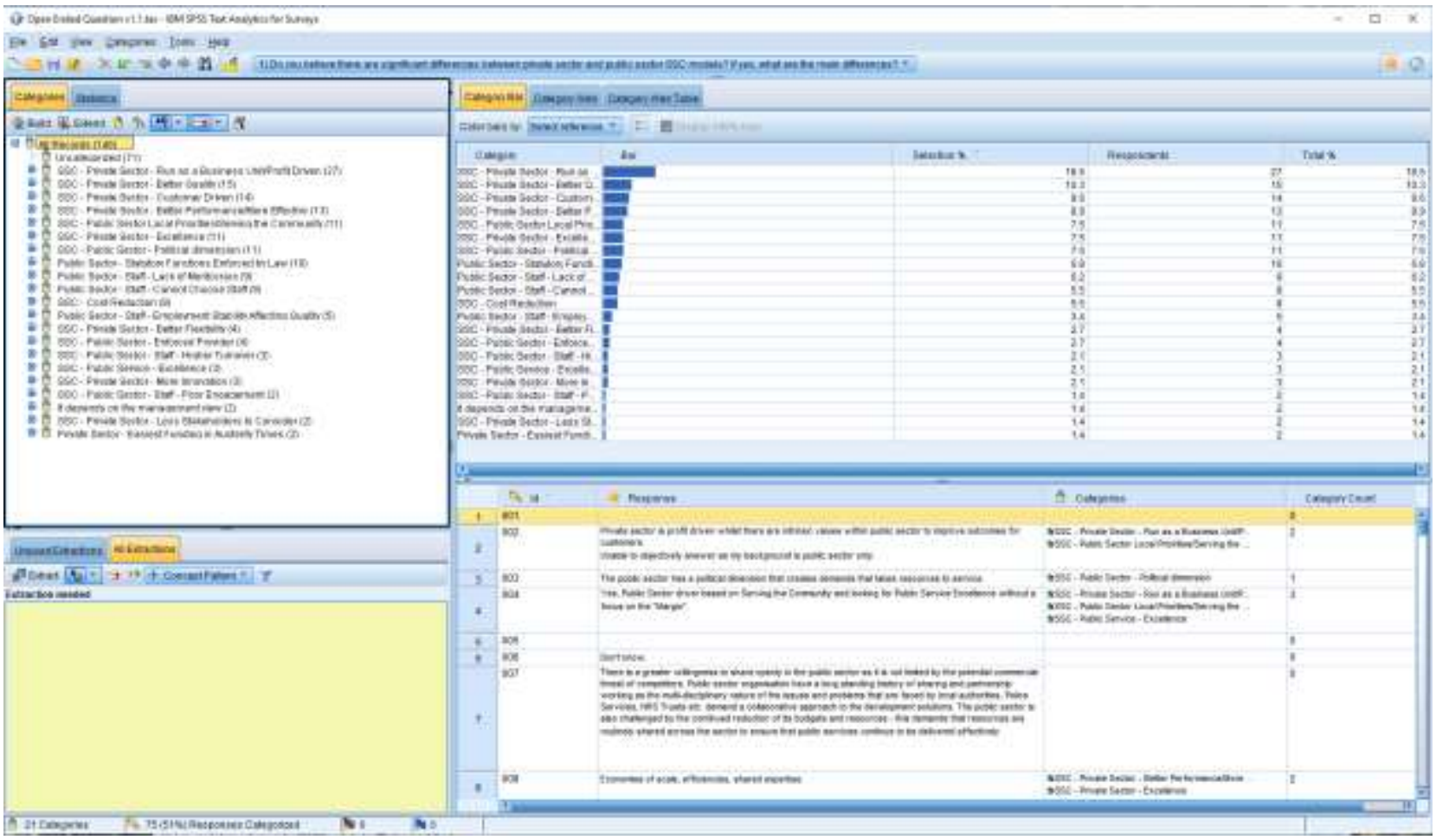

Among the 146 respondents, 86 (59\%) responded that they believe there are significant differences between the SSC of the public sector and the industry. Ten respondents (7\%) believe that there are no such differences, and 50 (34\%) remained neutral, choosing not to respond. The responses of those who believe there are significant differences were interpreted through the content analysis, and the results are discussed below.

As for the ten respondents who do not believe there are significant differences $(7 \%$ of the total), the reasons mentioned revolving around the understanding that the audience served by SSCs of Governments is so demanding as customers of the private SSCs. Also, state that the back-office services are similar many times in the two sectors. They mention that the public and private SSCs also work to satisfy customers, despite public sector bureaucracy ("Both have the customer as the heart," as one respondent said). 
The comments from respondents who believe there are significant differences resulted initially (after consolidation observing the principle of mutual exclusion mentioned earlier) in 21 categories of concepts, drawn from 154 excerpts of responses. These 21 categories analysed again and passed by one more consolidation that resulted in five broad categories whose details are found in Table 3.27 below. This table also presents the dimensions (of the organisational environment, external and internal) impacted by the concepts extracted from comments and arguments of the respondents. From an analysis based on these dimensions was possible to confirm that the SSC models of the industry and the public sector have significant differences. It was also found that may exist significant opportunities to improve the SSCs of the public sector, associated with relevant aspects such as service design, customer focus (outside-in), mission and governance of the SSC, management of the human factor in a service operation, and SSC business model. These differences are presented below and discussed in Chapter 4 .

Next, Table 3.27 below presents the following information: number of the category constituted from the analysis of content; names assigned to the five categories, representative of its content; summarisation of the characteristics of the categories; description of each category, associated with the respondents' comments; impacted organisational dimensions (external and internal); and several excerpts comments from respondents on the topics of each category. The categories with the bigger representativeness of respondents' comments are presented first. The information has been analysed in the light of the reviewed management theory about SSCs, service operations and organisations, and the results of these analyses are presented right after Table 3.27 below. 
Table 3.27 Categorisation of responses during the analysis of content

\begin{tabular}{|c|c|c|c|c|c|}
\hline$\#$ & $\begin{array}{l}\text { Categories of } \\
\text { differentiation } \\
\text { (public } x \\
\text { private SSCs) }\end{array}$ & $\begin{array}{l}\text { Main } \\
\text { characteristics of } \\
\text { the category }\end{array}$ & Category description & $\begin{array}{l}\text { Dimensions } \\
\text { impacted }{ }^{*}\end{array}$ & $\begin{array}{c}\text { Resp. } \\
\text { excerpts }\end{array}$ \\
\hline 01 & $\begin{array}{l}\text { Private Sector } \\
\text { SSC as a } \\
\text { service unit }\end{array}$ & $\begin{array}{l}\text { - Better quality } \\
\text { - Better } \\
\text { performance } \\
\text { - More effective } \\
\text { - Excellence } \\
\text { - Customer driven } \\
\text { - Innovation } \\
\text { - Better Flexibility }\end{array}$ & $\begin{array}{l}\text { Category that brings together the responses mentioning that the SSCs in the private sector have better } \\
\text { quality, and this sets them apart from the SSCs from the public sector. Brings together the answers that } \\
\text { still attach to private SSCs better performance and effectiveness when compared to the public sector } \\
\text { SSCs, and responses opining that the private SSCs feature excellence as a differential. Also, contains the } \\
\text { responses mentioning that the private SSCs are customer-oriented and most innovative. Moreover, } \\
\text { respondents in this category state that the private SSCs feature flexibility. }\end{array}$ & $\begin{array}{l}\text { - Service } \\
\text {-Service operation } \\
\text {-Service design } \\
\text { - Client } \\
\text { - Outside-in } \\
\text { - Governance } \\
\text {-Customer board }\end{array}$ & 46 \\
\hline 02 & $\begin{array}{l}\text { Public Sector } \\
\text { SSC as a (local) } \\
\text { public agency }\end{array}$ & $\begin{array}{l}\text { - Local priorities } \\
\text { - Serving the } \\
\text { community } \\
\text { - Statutory functions } \\
\text { enforced by law } \\
\text { - Enforced provider }\end{array}$ & $\begin{array}{l}\text { It groups the responses that include the obligation of attending local priorities as one of the main } \\
\text { limitations to the performance of the public SSCs. Also, contains the responses that the public SSCs are } \\
\text { intended to meet the needs of the community, and that this is an important distinction about the } \\
\text { private SSCs. It also includes the responses that present as one of the principal differences the fact of } \\
\text { the SSCs of the public sector must provide some services by law. Includes the responses that think that, } \\
\text { since the public SSCs have captive clients, who have not the option of being served by another provider, } \\
\text { they become less competitive. }\end{array}$ & $\begin{array}{l}\text { - Mission } \\
\text { - Legislation } \\
\text { - Bureaucracy } \\
\text { - Leadership } \\
\text { - Competition } \\
\text {-Monopoly }\end{array}$ & 39 \\
\hline 03 & $\begin{array}{l}\text { Private Sector } \\
\text { SSC as a } \\
\text { Business Unit }\end{array}$ & $\begin{array}{l}\text { - Run as a business } \\
\text { - Profit driven } \\
\text { - Easiest funding }\end{array}$ & $\begin{array}{l}\text { Encompasses the responses mentioning that the private SSCs are conducted as a business unit, unlike } \\
\text { the public SSCs. Also, groups the responses mentioning that private SSCs perform better because they } \\
\text { are oriented to profit, and this differentiates them from the public sector SSCs. Other respondents in } \\
\text { this category believe that in times of crisis, recently experienced, private SSCs have more access to } \\
\text { financial resources, and this results in better performance. }\end{array}$ & $\begin{array}{l}\text { - Business model } \\
\text {-Service fees } \\
\text {-Profit orientation } \\
\text { - Culture }\end{array}$ & 29 \\
\hline 04 & $\begin{array}{l}\text { Public Sector } \\
\text { SSC and the } \\
\text { public servant } \\
\text { dilemma }\end{array}$ & $\begin{array}{l}\text { - Lack of } \\
\text { meritocracy } \\
\text { - Cannot choose } \\
\text { staff } \\
\text { - Employment } \\
\text { stability } \\
\text { - Higher turnover } \\
\text { - Poor engagement }\end{array}$ & $\begin{array}{l}\text { This category groups the respondents who report that the lack of promotion and compensation based } \\
\text { on individual merit takes public SSCs having lower performance. It also includes the responses that } \\
\text { include the impossibility of choosing the right employees for the right positions as a major cause for } \\
\text { the underperformance of public SSCs. It also includes responses of those who understand that stability } \\
\text { in public service adversely affects the quality of service, and the responses indicating that the public } \\
\text { SSCs have staff turnover greater than private SSCs, hurting its performance. It also includes } \\
\text { respondents who understand that civil servants have comparatively a low level of engagement, which } \\
\text { negatively affects the performance of public SSCs. }\end{array}$ & $\begin{array}{l}\text { - Human Resources } \\
\text {-Motivation } \\
\text {-Leadership } \\
\text {-Team } \\
\text {-Culture }\end{array}$ & 27 \\
\hline 05 & $\begin{array}{l}\text { Public Sector } \\
\text { SSC and the } \\
\text { political } \\
\text { environment }\end{array}$ & $\begin{array}{l}\text { - Political } \\
\text { interference } \\
\text { - More stakeholders }\end{array}$ & $\begin{array}{l}\text { Consists of responses that the political aspects take the public SSCs having an inferior performance. } \\
\text { This group is also home to the responses of those who believe that the public SSCs are affected } \\
\text { negatively by having more stakeholders than the private SSCs. }\end{array}$ & $\begin{array}{l}\text { - Political } \\
\text { environment and } \\
\text { power } \\
\text { - Alignment of } \\
\text { interests } \\
\text { - Leadership } \\
\end{array}$ & 13 \\
\hline
\end{tabular}

Note: * The impacts are explained and discussed in Chapter 4 . 


\subsubsection{Results of the Analysis of Categories, Based on the Dimensions Impacted}

The analysis developed and presented here seeks to explain the nature of the concepts mentioned by respondents (see Table 3.27 above) and how they impact the SSCs. The resultant five categories are candidates of been transformed into actions for perfect the public SSC model, and later were submitted to the Action Learning Set (see Section 3.13).

Category 01 Private Sector SSC as a service unit. This category represents the set of responses from those who believe that the SSCs from the industry offer services with better quality, better performance, more effectiveness, excellence, and that they are more oriented to customers, innovative and flexible. All these concepts are linked to attributes of services that are defined during the design of the service that will be offered by a service operation (Johnston \& Clark, 2001; Frei, 2008), such as an SSC. Although these characteristics may be present both in public and private SSCs, this research showed that the perception of the respondents is that the industry SSCs are more skilled in these matters. A possible interpretation in the light of the theory is that the starting point for the design of the SSC operation in the industry tends to be more 'outside-in', that is, tends to start at the clients' view, and the attributes that lead to satisfaction with the service (see Table 3.28 below) tend to be adopted more efficiently by the private SSCs. 
Table 3.28 Factors for design and analysis of service operations

\begin{tabular}{|c|c|}
\hline 12 Factors & Description of the Factors \\
\hline Availability & $\begin{array}{l}\text { Availability of the services, of the staff and facilities, offered to the } \\
\text { client, escalation. }\end{array}$ \\
\hline Reliability & Performance consistency of services and staff. Accuracy of information. \\
\hline Feature & Adequacy of services to customer needs. \\
\hline Control & Security of the client's possessions while benefits from the service. \\
\hline Service price & Price suitability for service. \\
\hline Aesthetics & $\begin{array}{l}\text { The extent to which the service package components are in line or to } \\
\text { the satisfaction of the client. }\end{array}$ \\
\hline Responsiveness & $\begin{array}{l}\text { Speed and service delivery punctuality. Prompt response to client } \\
\text { requests. }\end{array}$ \\
\hline Communication & $\begin{array}{l}\text { Ability to communicate the service in an intelligible way. Clarity and } \\
\text { precision in verbal and written information. }\end{array}$ \\
\hline Competency & $\begin{array}{l}\text { Skill, expertise, and professionalism. Adoption of correct procedures } \\
\text { and correct execution of the instructions of the clients and regulators. }\end{array}$ \\
\hline Service & $\begin{array}{l}\text { Organisation for service and help to the client. The staff passes the } \\
\text { impression of being interested in the client, showing willingness in } \\
\text { serving. }\end{array}$ \\
\hline Attitude & $\begin{array}{l}\text { Reaction to adversity, receptivity to criticism to the services, attitude to } \\
\text { promote changes, proactivity, "extra-mile/ best effort." }\end{array}$ \\
\hline Flexibility & $\begin{array}{l}\text { Willingness on the part of the company to supplement or amend the } \\
\text { nature of service to meet the needs of clients. }\end{array}$ \\
\hline
\end{tabular}

Source: Adapted from Johnston \& Clark (2001).

According to Johnston \& Clark (2001), the 12 attributes presented in the Table 3.28 above express the various characteristics of a service and if carefully defined and practiced from the vision of the client (outside-in), raise the chances of success of the service operation. Another important aspect to consider is the governance of the SSC, which must be administered by its clients, usually through a Client Board or equivalent 
(Ashkenas, 1995). This is one of the core principles of the constitution of an SSC (Schulman et al., 1999; Bergeron, 2002; Quinn et al., 2000).

Category 02 Public Sector SSC as a (local) public agency. This category includes respondents who consider that the fact that the SSCs of governments having to meet local priorities, provide the services prescribed by law and as they are of compulsory use of its clients (which cannot receive the service from another provider), makes these SSCs comparatively less competitive. Although research shows that this is a reality, this need not necessarily be that way. The search for a public service of better quality and more suitable for citizens is at the core of the principles of New Public Management (Gershon, 2004; Schwarz, 2014; Tomkinson, 2007), adopted by many governments (such as USA and UK) as the driver of adoption of SSCs as a solution to support services. The correct definition of SSC's mission (considering the best use of public money and the outside-in design discussed in the previous category), as well as the entrepreneurial attitude of leadership, can soften the negative impact that the bureaucracy and the legislation provoke in the effectiveness of the public SSCs. They can also mitigate the negative consequences that the 'monopoly,' or lack of competition leads on mechanisms and encouragement for the continuous improvement of public SSCs, which have comparatively lower quality, in the opinion of survey respondents. It is also important to recognize that the law exerts a counterforce to the sound performance of the public service, in general, because unlike the private sector, a public service, usually, can only be provided if there is a law that permits, and with the authorized content and form (Denhardt \& Denhardt, 2000). While the industry has greater freedom since, roughly, the service can be provided if there is no law that prohibits. 
Category 03 Private Sector SSC as a Business Unit. This category brings together those respondents who claim that the industry SSCs feature better overall quality because they are conducted as a business, profit-oriented and can fund more easily, especially in times of crisis. Although this reality seems to be more common in private SSCs, it is often feasible also for public SSCs. The SSCs of the public sector also can adopt the charging for the offered services and use the resources obtained in the maintenance of services with high level of quality, working as a public agency or in another legal configuration, as a public-private partnership, for example (Schwarz, 2014; Gould et al., 2007; Janssen \& Joha, 2010). Another essential factor for this, is the formation of a culture specific for the SSC, focused on excellence and best practices in services with inter-sector benchmarks established, whenever the type of service offered allows (Keuper \& Lueg, 2013; Schwarz, 2014).

Category 04 Public Sector SSC and the public servant dilemma. Respondents in this category attribute the comparatively lower performance of SSCs of the public sector to the human factor, as the absence of meritocracy as criteria for pay and promotion; the fact that many public SSCs cannot choose their staff; job stability as a sponsor of accommodation and not encouraging the continued development; a higher turnover of staff in the SSCs, which reduces the average level of competency of employees and their condition to provide a better service; and a minor commitment of the public officials pro-excellence of service. Since these causes are difficult to change in the public sector, the management theory offers alternatives and best practices in areas of study as motivation, leadership, teamwork and organisational culture as possible remedies for overcoming these barriers to quality of service (Schwarz, 2014; Baldwin, 1987; Gershon, 2004; Robbins, 2011). Even with these difficulties, it is possible to establish a context of 
centre of excellence in public SSCs (Ulbrich, 2010) and, in general, this is more linked to the willingness and management ability of the managers than the 'corporative' formal systems (Schwarz, 2014; Denhardt \& Denhardt, 2000).

Category 05 Public Sector SSC and the political environment. This category is formed by the comments that affirm that the political interference affects the performance of the public SSCs negatively, as well as for the comments that attribute this level of inferior performance to have been to work with a number expressively bigger of stakeholders in the public context. To deal with these opposing forces and to enable the management of expectations and demands of stakeholders, management theory offers solutions for understanding the power and political contexts (Robbins, 2011), as well as successful professional managing these forces. It also provides extensive theory and case studies for effective mapping and stakeholder management (Klefsjö et al., 2008; Robbins, 2011) and effective organisational communication (Robbins, 2011).

This analysis identified significant differences in the context of public and private SSCs, which brings numerous challenges to public sector managers to establish and manage the SSCs. From the results of this analysis we can infer that the questioning that the SSC models of public sector require specific settings and not simply be copied from the private sector-corresponding to the research question 2 (RQ2) “Do Shared Services Centres of the public sector differ from the SSCs of the private sector? In which way?"is well-founded. These results were submitted to the Action Learning Set for a reality check and refinement, which are discussed in the next section; and the final discussion and conclusions of this theme are carried out in Chapter 4. 


\subsection{The Action Learning Set (ALS)}

Action Learning Sets are small groups (usually 5 to 8 people) used as a means for learning and solving complex problems. ALS shares, discusses and helps solve problems brought by its members, and therefore, is a learning community (Pedler, 2008). The central role of ALS is not to find a solution for the problems presented, but help the member who has the problem to find the solution and grow. Herr \& Anderson (2014) defend that the ALS is a way to approach the researcher of those who are the object of research. According to Pedler (2012), the ALS is one of four interrelated elements of the action learning, along with the person, the problem and the action. Among the benefits of the ALS, I mention: it offers an opportunity for personal growth; it improves problemsolving skills; it helps the member with a problem to deal with complex tasks; the participant with an issue is challenged and stimulated by the group; it contributes clarifying the ideas and analyses. The ALS of this research was created following recommendations of Pedler (2012), Coghlan \& Brannick (2014), and Greenwood \& Levin (2006) as, for example, confidentiality, commitment, common interest, cooperation, mutual development, helpful and democratic environment, among others. Thus, the survey results were discussed with experienced managers and experts, members of the ALS, and transformed into applicable knowledge to meet the reality of Shared Services Centres. Because of the need of having participants from several countries in my ALS, a virtual ALS (Pedler, 2011), asynchronous, was used for this inquiry, as detailed in the following.

\subsubsection{Composition of the Action Learning Set for this Thesis}

Since the survey respondents were from several countries, mainly the UK, the USA, Canada, and Brazil, I tried to form the ALS with experts from the same countries, as this 
could help in a more precise understanding of the survey results, and an improved outcome for the SSC community.

Figure 3.7 below shows a schematic overview of the ALS of this thesis and, afterward, I explain its composition, and how it was formed.

Figure 3.7 Schematic View of the Action Learning Set for this DBA Thesis

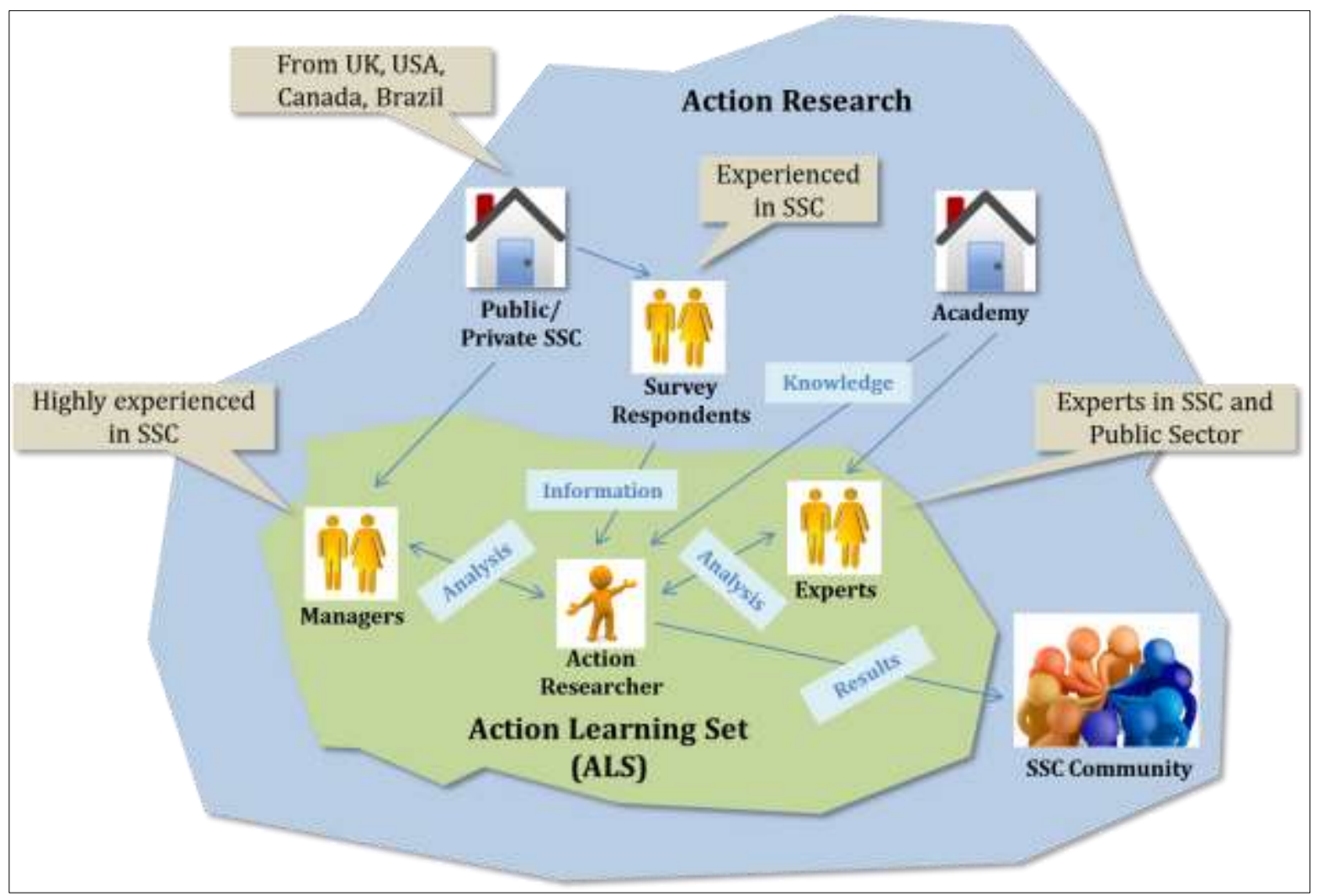

The ALS was composed of seven members in total, five public SSCs managers, one private SSCs manager, and one doctored professor who specialises in public service. To constitute the ALS, first, it was identified which competencies should be present in the ALS and next (solid knowledge in SSC and public sector), 14 potential participants, with the necessary skills, were invited based on a previous search on LinkedIn, and my contacts. Thus, seven managers and experts from Brazil, Canada, the USA, and the UK (same countries of much of survey respondents) confirmed their interest, willingness, 
and availability to take part, and became members of the ALS. Then, the ALS members received the details of the ALS objectives, and instructions for their effective contribution (see Appendix D).

\subsubsection{How the Action Learning Set Worked}

The ALS working method was based on questioning and reflection (Pedler, 2012), and two rounds of discussion were applied, within 40 days. Because of participants agenda limitation, six of seven ALS members joined the first round of debate, and six the second (with one different participant, comparing to the first round). Figure 3.8 below shows how the ALS worked and, the following, I explain in more details.

Figure 3.8 How the Action Learning Set Worked

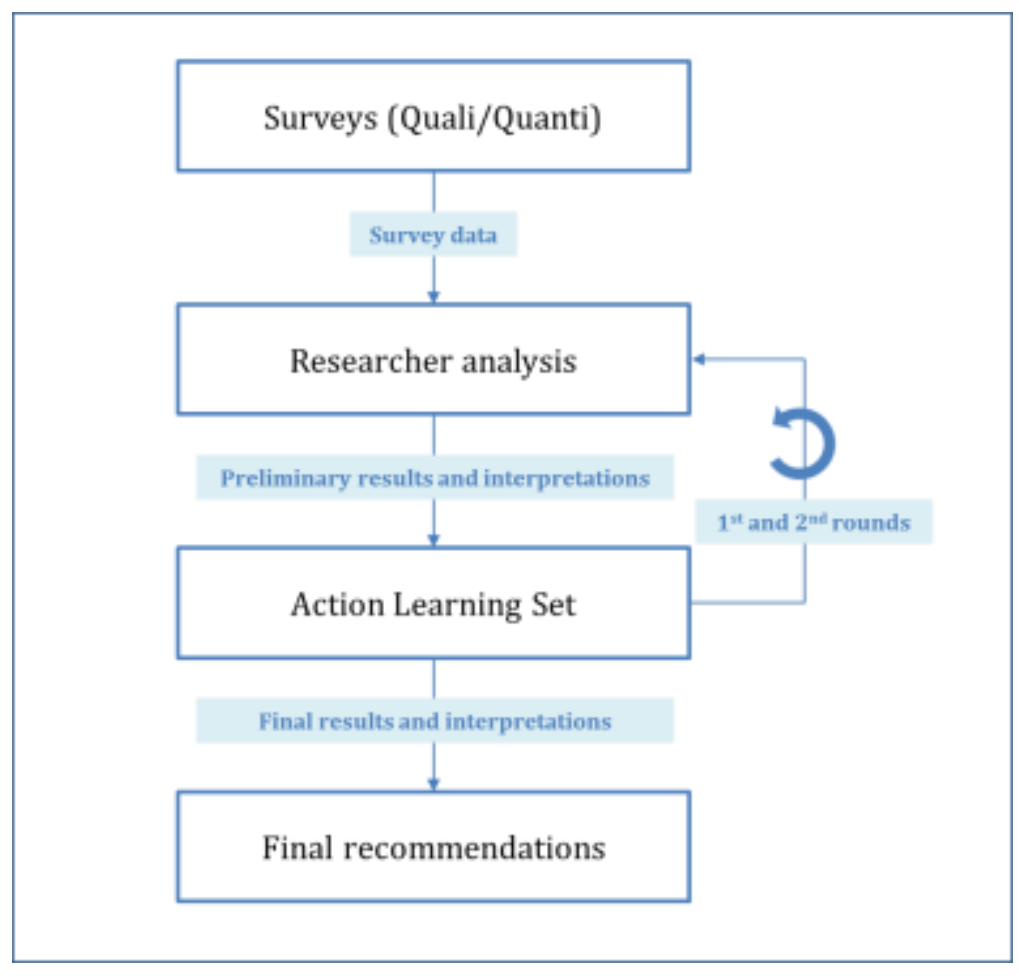

In the first round, submitted to the ALS were the results of the quantitative and qualitative approaches (see Sections 3.11 and 3.12), properly consolidated and with my 
initial interpretation of them considering the theory; step further discussed in the next section.

The five categories of findings that emerged in the qualitative approach (see Section 3.13.3.1 below), along with the results obtained in the quantitative approach, were analysed and interpreted by me, with the support of the literature reviewed. These original interpretations, discussed the following, were then submitted to the ALS. Each ALS member evaluated the findings and my initial interpretations and made comments agreeing or disagreeing, and offering new elements of SSC or Public-Sector context, and possible practical application. The responses were then consolidated and submitted by me to a new treatment of content analysis using the same technical protocol discussed in Section 3.12. The only difference to that protocol is that, although I had access to other more prominent software, as NVivo and SPSS Text Analytics, I decided to usesuccessfully—the QDA Miner Lite. This free software proved to be friendly, lightweight, flexible and with appropriate resources to categorisations, coding, and analysis. Because of the interaction of ALS (first round) and results of the ALS content analysis, after treatment and consolidation of the suggestions received, emerged eight measures for proper adaptation and improvement of public SSCs. These eight measures, presented and discussed in Section 4.3, were then submitted to the ALS (second round) for last reflections and final considerations. In the next section, I show the process mentioned above in more details and explain how I understood and interpreted the ALS discussions.

\subsubsection{Dynamics and Contributions of the Action Learning Set}

The starting point for working with the ALS were the results obtained from the quantitative and qualitative approaches. In Sections 3.11 and 3.12, I explain how I 
performed the interpretations and analyses that led to the preliminary results submitted to ALS. Next, I discuss how the interaction with ALS members occurred, illustrate their reactions to the initial results, and exemplify their contributions, disagreements, and concordances. Besides, I explore how I applied the interpretation (with the support of content analysis) to the responses of ALS members in the first round of discussions to reach the recommendations proposed by this thesis, refined in the second round of discussions.

\subsubsection{First Round of Discussions}

In the first round, the preliminary results of the survey (see Sections 3.11 and 3.12), were sent to the members of the ALS, as described in the items (A), for the qualitative part of the survey, and (B), for the quantitative part of the survey, as presented below.

(A) Key findings of the qualitative part of the survey (Appendix D - Example 2, Item 1) - The responses of the qualitative part were analysed with support of content analysis, resulting in five categories of concepts or findings: Category 01 Private Sector SSC as a service unit; Category 02 Public Sector SSC as a (local) public agency; Category 03 Private Sector SSC as a Business Unit; Category 04 Public Sector SSC and the public servant dilemma; Category 05 Public Sector SSC and the political environment. Next, I present my initial interpretation for each of these five categories, in the light of the theory, which was submitted to the analysis of ALS members.

Category 01 Private Sector SSC as a service unit - This category represents the set of responses from those who believe that the SSCs from the industry offer services with 
better quality, better performance, more effectiveness, excellence, and that they are more oriented to customers, innovative and flexible.

My initial interpretation - A summarised preliminary interpretation, in the light of the theory, is that the starting point for the design of the SSC operation in the industry tends to be more 'outside-in.' That is, tends to start at the clients' view, and the attributes that lead to satisfaction with the service (Table 3.28 above) tend to be adopted more efficiently by the private SSCs.

Category 02 Public Sector SSC as a (local) public agency - This category includes respondents who consider the fact that the SSCs of governments having to meet local priorities, provide the services prescribed by law and as they are of compulsory use of its clients (which cannot receive the service from another provider), makes these SSCs comparatively less competitive.

My initial interpretation - A summarised preliminary interpretation, in the light of the theory, is that the correct definition of SSC's mission (considering the best use of public money and the outside-in design discussed in the previous category), as well as the entrepreneurial attitude of leadership, can soften the negative impact that the bureaucracy and the legislation provoke in the effectiveness of the public SSCs.

Category 03 Private Sector SSC as a Business Unit - This category brings together those respondents who claim that the industry SSCs feature better overall quality because they are conducted as a business, profit-oriented and can fund more easily, especially in times of crisis.

My initial interpretation - A summarised preliminary interpretation, in the light of the theory, is that the SSCs of the public sector also can adopt the charging for the offered 
services. They can use the resources obtained in the maintenance of services with high level of quality, working as a public agency or in another legal configuration, as a publicprivate partnership, for example. Another essential factor for this is the formation of a culture specific for the SSC, focused on excellence and best practices in services with inter-sector benchmarks established, whenever the type of service offered allows.

Category 04 Public Sector SSC and the public servant dilemma - Respondents in this category attribute the comparatively lower performance of SSCs of the public sector to the human factor, as the absence of meritocracy as criteria for pay and promotion; the fact that many public SSCs cannot choose their staff; job stability as a sponsor of accommodation and not encouraging the continued development; a higher turnover of staff in the SSCs, which reduces the average level of competency of employees and their condition to provide a better service; and a minor commitment of the public officials pro-excellence of service.

My initial interpretation - A summarised preliminary interpretation, in the light of the theory, is that since these causes are difficult to change in the public sector, the management theory offers alternatives and best practices in areas of study as motivation, leadership, teamwork and organisational culture as possible remedies for overcoming these barriers to quality of service.

Category 05 Public Sector SSC and the political environment - This category is formed by the comments that affirm that the political interference affects the performance of the public SSCs negatively, as well as for the comments that attribute this level of inferior performance to have been to work with a number expressively bigger of stakeholders in the public context. 
My initial interpretation - A summarised preliminary interpretation, in the light of the theory, is that to deal with these opposing forces and to enable managing expectations and demands of stakeholders, management theory offers solutions for understanding the power and political contexts, as well as successful professional managing of these forces. It also provides theory and case studies for effective mapping and stakeholder management and effective organisational communication.

ALS members were asked to review these five categories and my initial interpretations and answer the open questions asked (Appendix D - Example 2), based on their experiences and expertise in SSC and the public service. Each ALS member sent their responses by email, and I performed an analysis to consolidate the results of the first round of discussion; thus, I created a control table of concordance by a member (Table 3.29 below). As we can see in that table, most of the ALS members agreed with my initial interpretations, of the qualitative part, with two exceptions discussed after the table.

Table 3.29 Agreement Table - Round One

\begin{tabular}{|l|c|c|c|c|c|c|}
\hline Key findings & M1 & M2 & M3 & M4 & M5 & M6 \\
\hline $\begin{array}{l}\text { Category 01 Private Sector SSC as a } \\
\text { service unit }\end{array}$ & A & A & D & A & A & A \\
\hline $\begin{array}{l}\text { Category 02 Public Sector SSC as a (local) } \\
\text { public agency }\end{array}$ & A & A & A & P & $?$ & P \\
\hline $\begin{array}{l}\text { Category 03 Private Sector SSC as a } \\
\text { Business Unit }\end{array}$ & A & A & A & D & $?$ & A \\
\hline $\begin{array}{l}\text { Category 04 Public Sector SSC and the } \\
\text { public servant dilemma }\end{array}$ & A & A & A & A & A & A \\
\hline $\begin{array}{l}\text { Category 05 Public Sector SSC and the } \\
\text { political environment }\end{array}$ & P & A & P & P & $?$ & P \\
\hline
\end{tabular}

Legend: $\mathbf{A}=$ Agree; $\mathbf{D}=$ Disagree; $\mathbf{P}=$ Partially agree; = Comments with no clear answer; $\mathbf{M}=$ Action learning set member.

In the first exception, Member 3 commented for Category 01 (Table 3.29 above), differently from the others, that he did not agree that private SSCs tend to be more 
'outside-in' than public SSCs because, in his view, both are similar operations (what changes is the context). He said, "No. In my experience, the design stage of both public and private shared services starts with the objectives that the organisation can achieve by setting up (or purchasing) the shared services organisation... the difference is who receives the benefit from shared services and their ability to sponsor the changes that, by definition, need to occur introducing shared services. In the industry, shared services organisations are typically driven by the CEO or the senior executive team..." In the second exception, for Category 03, the ALS Member 4 not fully agreed that public SSCs can charge for services offered because, he thinks, governments follow a no economic logic. In his words, "Not in principle... As we know, in Brazilian and even Latin American experience, the integration and sharing of services of the private field make cut-outs that adhere to an economic logic."

About the ALS members who agreed with my preliminary interpretations in the five categories of key findings, to illustrate their arguments, I captured a few comments, which can be seen in Table 3.30 below. 
Table 3.30 ALS Members Agreeing with my Initial Interpretation of the Key Findings - Round One

\begin{tabular}{|c|c|}
\hline Key findings & Example of ALS memberagreement \\
\hline $\begin{array}{l}\text { Category } 01 \text { Private Sector SSC as a } \\
\text { service unit }\end{array}$ & $\begin{array}{l}\text { "Public sector organisations are inherently inward looking (despite what they say!). Generally, } \\
\text { they do not have to market their services, or compete with others, and can rely on regulations } \\
\text { to make customers comply." (ALS Member 1]. "It would be required that public services, } \\
\text { usually fragmented in various areas... were performed as public policy, integrated and as such } \\
\text { they would be seen and perceived by the population." [ALS Member 4]. }\end{array}$ \\
\hline $\begin{array}{l}\text { Category } 02 \text { Public Sector SSC as a (local) } \\
\text { public agency }\end{array}$ & $\begin{array}{l}\text { 'Yes, your interpretation is correct from my experience. Just the act of separating the service } \\
\text { from its 'home' organization increases organizational focus, exposes the service to competitive } \\
\text { market forces and increases expectations of the 'home' organization." [ALS Member 3]. }\end{array}$ \\
\hline $\begin{array}{l}\text { Category } 03 \text { Private Sector } 55 C \text { as a } \\
\text { Business Unit }\end{array}$ & $\begin{array}{l}\text { "In the federal government [USA] we consider an organization a SSC if they have a 'Charge } \\
\text { back' model. SSCs can serve customers from other organitations or just components within the } \\
\text { same organization (all on a fee for service basis). Without the 'charge back' model, it would } \\
\text { really be consolidating administrative functions, which is not part of the federal SSCS. Having a } \\
\text { charge back model is critical and having a 'data focus' on performance and compliance with } \\
\text { service level agreements will help set the right 'culture'. [ALS Member 6]. }\end{array}$ \\
\hline $\begin{array}{l}\text { Category } 04 \text { Public Sector SSC and the } \\
\text { public servant dilemma }\end{array}$ & $\begin{array}{l}\text { I agree although to fully address the issues more specific solutions would need to be } \\
\text { recommended. Issues with performance include a lack of ability to fully hold employees } \\
\text { accountable, employees not feeling connected to the mission of the organization(s) they serve, } \\
\text { and the transactional work they perform is very tedious and repetitive with very little } \\
\text { recognition for your efforts (which leads to the turnover)." [ALS Member 6]. "Organisations } \\
\text { remain risk averse. No one is prepared to take a chance (primarily as it is public money). } \\
\text { Organisations need to look to be more innovative, make greater use of technology to drive } \\
\text { down costs (being acutely aware of potential significant upfront costs)" [ALS Member S]. }\end{array}$ \\
\hline $\begin{array}{l}\text { Category } 05 \text { Public Sector SSC and the } \\
\text { political environment }\end{array}$ & $\begin{array}{l}\text { "As I mentioned, the SSC is at the mercy of the government, even being an administrative area } \\
\text { that should be perennial. if the SSC is not placed as a public policy for cost reduction... every } \\
\text { new mandate the new administration will not support the model." [ALS Member 2]. }\end{array}$ \\
\hline
\end{tabular}

On the partial concordances, shown in the Table 3.30 above, an illustrative example is that of Member 4, for Category 02. He said, "I do not fully agree because bureaucracy and legislation are previously determined by fragmented organisational culture, by departmental obedience to patronage modes of political action. Bureaucracy and legislation are not decisive." In another example, Member 6 commented on this same category, "In my experience, federal SSCs in the US have rigorous federal regulations and requirements that can be burdensome. They also cannot work independently in that their 'parent' agency may set priorities that may limit their focus area (given the mission of the parent agency differs from that of the SSC)."

The high number of concordances supported my initial interpretations. However, the comments and reflections submitted by the ALS members brought new relevant elements (and 'new readings' of the reality) and led me to do a new analysis of content, 
now of the reflections and other comments from the ALS. As well as a new general analysis of all the findings so far, with the goal of transforming them into actionable knowledge (e.g. practical actions for the SSC community). This new general analysis was made considering four main sets of information: the seven components of the conceptual model; the five criteria resulting from the qualitative part of the survey; the reflections of the ALS members; and the revised literature. The new general analysis resulted in six measures that the SSCs of the public sector can adopt to perfect its service operations, presented in the Table 3.31 below, which were submitted to the ALS for validation in the second round of discussions. 


\begin{tabular}{|c|c|c|}
\hline \multirow[b]{2}{*}{$\begin{array}{l}\text { Six Proposed Measures } \\
\text { (see Section 4.3) }\end{array}$} & \multicolumn{2}{|r|}{ Examples of research findings supporting the six proposed measures } \\
\hline & $\begin{array}{l}\text { Categories resulting } \\
\text { from the Survey (see } \\
\text { above) }\end{array}$ & Action Learning Set Insights \\
\hline $\begin{array}{l}\text { Measure } 1 \text { - Create its own } \\
\text { service culture }\end{array}$ & Categories 04 and 05 & $\begin{array}{l}\text { "Don't try to make this SSC become just another arm of the bureaucracy. Give it different targets which derive from } \\
\text { customers, and not from 'these are everybody's targets.' Allow the SSC to develop its own culture." [Member 1]. } \\
\text { "Implement management practices that provide the leadership which will create a customer service oriented culture. An } \\
\text { adequate level of segregation from the public service for this culture to develop." [Member 3]. }\end{array}$ \\
\hline $\begin{array}{l}\text { Measure } 2 \text { - Steering by its } \\
\text { customers (client focus) }\end{array}$ & Category 02 & $\begin{array}{l}\text { "So, the objective is to be counter-cultural, to put the bureaucracy aside and do what the customer wants/needs." } \\
\text { [Member 1]. "SSCs could set up a governance board that helps to decide how investments are made (e.g," new } \\
\text { functionality, upgrades, new customers, etc.) and ensure their clients have more of a voice." [Member 6]. }\end{array}$ \\
\hline $\begin{array}{l}\text { Measure } 3 \text { - Develop an } \\
\text { entrepreneurial leadership }\end{array}$ & Categories 03 and 04 & $\begin{array}{l}\text { "Essentially, leadership is required, preferably from the person or people who have experience of running a business in } \\
\text { the real world. Get good leadership. That may not come from a traditional public-sector background." [Member 1]. "The } \\
\text { leader must have the vision of providing services." [Member 2]. "A management team that is capable of running the SSC } \\
\text { as an independent not for profit organisation... provide the leadership which will create a customer service oriented } \\
\text { culture." [Member 3]. }\end{array}$ \\
\hline $\begin{array}{l}\text { Measure } 4 \text { - Bring some } \\
\text { people from the industry to } \\
\text { the public SSC }\end{array}$ & Category 01 & $\begin{array}{l}\text { "The attitudes required are to be found in the private sector (but why would they want to work in a bureaucracy?) and in } \\
\text { a minority of public sector staff." [Member 1]. }\end{array}$ \\
\hline $\begin{array}{l}\text { Measure } 5 \text { - Public SSC as a } \\
\text { State Policy }\end{array}$ & Categories 03 and 05 & $\begin{array}{l}\text { "There is a need to agree strategies, objectives, boundaries, and then let the managers manage. Politicians have to } \\
\text { recognise they are not managers of these business units." [Member 1]. "Implementation of policies that are more } \\
\text { perennial to mature the practices placed in the public sector." [Member 2]. "Difficult for local authorities with elections } \\
\text { which can frequently change the political make-up of the Council. Different political parties could have different } \\
\text { priorities? Services are then altered or even stopped which may not be to the customers good. Only accountable at the } \\
\text { next election." [Member 5]. }\end{array}$ \\
\hline $\begin{array}{l}\text { Measure } 6 \text { - Develop } \\
\text { organisational resilience }\end{array}$ & Categories 02 and 03 & $\begin{array}{l}\text { "Compensation programs that are aligned with the SSC business rather than the "home" organisations business. Effective } \\
\text { Customer Service program. Effective Governance and Relationship Management program. Segregation of the SSC from } \\
\text { the client public service." [Member 3]. "... moving federal SSCs outside of their existing federal agencies to another agency } \\
\text { that focuses on the delivery of administrative services will help to ensure mission alignment." [Member 6]. }\end{array}$ \\
\hline
\end{tabular}

Note: Section 4.3 discusses the seven components of the conceptual model, associating them to the proposed measures and highlighting the reviewed literature most relevant. 
(B) Key findings of the quantitative part of the survey - In the first round, ALS members were also sent the quantitative research results that needed to be discussed using the expertise of the ALS (Appendix D - Example 2, Item 2). That is, the proposed conceptual model was empirically validated, and the relationship among its variables was confirmed (Section 3.11). Meanwhile, the relationships of the Market Orientation variable brought some surprises that I could explore with the ALS experts. For this, I submitted the following question to them (Appendix D - Example 2): "The results of quantitative research (extracted by multivariate data analysis) confirmed that there is a positive relationship between the elements (variables) of the proposed model, validating the model. However, the relationship between Excellence and Market Orientation proved to be much weaker than the relationship between Excellence and Performance. Also, the relationship between Market Orientation and Performance was weak. In both cases, for public and private SSCs. An initial interpretation could be that the degree of market orientation (customer-orientated SSC) does not influence much on the SSC performance and that the level of excellence is more crucial in this. What is your opinion about this?"

Five out of the six managers and experts engaging in the first round disagreed with my interpretation. They argued that, in general, Market Orientation is key to the success of any SSC, and the only one who agreed (Member 6), understands that excellence already has built a significant portion of customer orientation. The following are three examples of disagreeing comments. Member 1: "I think market orientation is key to success. There is no point in glorifying excellence if you do not understand what it looks like (to stakeholders!!).” Member 3: "Excellence and performance get the SSC into the game. Market orientation keeps you in the game. In other words, without Market Orientation, an SSC will not be able to sustain performance." Member 5: "I am of the view 
that they all impact/influence on a similar level. Organisations are starting to get savvier and need to take all through equally."

At the end of the first round of the ALS discussions, I got the following products: (A) in the qualitative part of the research - validation of the five categories of survey findings, and my initial interpretation for them, with relevant comments and insights from the ALS members. New general analysis of available data, which resulted in the six measures discussed above. (B) in the quantitative part of the research - new insights on Market Orientation, Performance, and Leadership, useful for interpreting the quantitative survey results.

\subsubsection{Second Round of Discussions}

I split the interaction with the ALS members in the second round, as I did in the first round, in two parts (A) Qualitative; and (B) Quantitative, which I discuss below.

(A) Qualitative part - I have consolidated the comments and suggestions from the ALS participants, obtained during the first round, making new content analysis, considering the studied conceptual lenses. Then, I proposed to ALS, for the second round of discussion (Appendix D - Example 3), for the qualitative part of the survey, the six measures for a proper adaptation and improvement of public SSCs that are listed here and discussed in Chapter 4, along with recommendations for implementation: 1 . Create its own service culture; 2. Steering by its customers (client focus); 3. Develop an entrepreneurial leadership; 4. Bring some people from the industry to the public SSC; 5 . Public SSC as a State policy; 6. Develop organisational resilience. These measures were conceived from the discussions of the five categories generated during the content analysis and the reflection on the validation of the conceptual model in conjunction with 
the ALS (first round). I sought to gather, analyse and consolidate the individual contributions of the ALS participants, and submit them to the group. Thus, the six measures, generated as a result of the first round of discussion, were sent to ALS for further consideration, which ratified it (Table 3.32 below) with only two members (5 and 7) disagreeing with the Measure 5 - Public SSC as a State policy, because they understand that the SSC should not be deployed 'by imposition.'

Table 3.32 Agreement Table - Round Two

\begin{tabular}{|c|c|c|c|c|c|c|}
\hline Key findings & M1 & M2 & M3 & M5 & M6 & M7 \\
\hline Measure 1 - Create its own service culture & A & A & A & A & A & A \\
\hline $\begin{array}{l}\text { Measure } 2 \text { - Steering by its customers } \\
\text { (client focus) }\end{array}$ & A & A & A & A & A & A \\
\hline $\begin{array}{l}\text { Measure } 3 \text {-Develop an entrepreneurial } \\
\text { leadership }\end{array}$ & A & A & A & A & A & A \\
\hline $\begin{array}{l}\text { Measure 4-Bring some people from the } \\
\text { industry to the public SSC }\end{array}$ & A & A & A & A & A & $\mathrm{N}$ \\
\hline Measure 5 - Public SSC as a State Policy & A & A & A & D & A & D \\
\hline $\begin{array}{l}\text { Measure } 6 \text {-Develop organisational } \\
\text { resilience }\end{array}$ & A & A & $\mathrm{N}$ & A & A & A \\
\hline
\end{tabular}

Legend: $\mathbf{A}=$ Agree; $\mathbf{D}=$ Disagree; $\mathbf{N}$ = Neutral; $\mathbf{M}$ = Action learning set member.

In the words of Member 7: "Shared services are logical, if they're not then they would not happen regardless of legislative drivers... you cannot force people/organisations to work together... if you get unwilling partners working together, it will be a huge costly failure despite legislation. Of course, if it was a public-private venture and a highly leftwing administration came into power at any level, then they could philosophically go backwards... but the world is too complex for the 1970s these days."

Table 3.33 the following shows some examples of comments of those who agreed with the proposed six measures. 
Table 3.33 ALS Members Agreeing with the Six Measures Proposed

\begin{tabular}{|c|c|}
\hline Key findings & Example of ALS member agreement \\
\hline $\begin{array}{l}\text { Measure } 1 \text { - Create its own service } \\
\text { culture }\end{array}$ & $\begin{array}{l}\text { The incentive to provide a good + service to customers is enhanced through charging as the } \\
\text { customer can withhold payment; where competition exists the customer can move to another } \\
\text { provider." [Member } 1 \text { ]. I do strongly agree that it has to have its own culture but also one that } \\
\text { meets the overall culture of the sector it is servicing-" [Member } 7 \text { ]. }\end{array}$ \\
\hline $\begin{array}{l}\text { Measure } 2 \text { - steering by its } \\
\text { customers (client focus) }\end{array}$ & $\begin{array}{l}\text { 'The sustenance of a SSC to be 'everlasting,' must value highly the clients' needs, and not just cost } \\
\text { reduction, Over the years, as the SSC becomes more mature, the recurring cost reduction in } \\
\text { almost null." [Member } 2 \text { ]. 'One element through which SSC's deliver value is through the } \\
\text { efficiency provide by scale. This scale is obtained through the standardization of work processes } \\
\text { and items purchased. The natural desire of customers is to avoid this change. While I agree it is } \\
\text { essential that the customers have input into the SSC's strategic direction this must also be } \\
\text { balanced off by the SSC imperative to provide value." [Member 3]. }\end{array}$ \\
\hline $\begin{array}{l}\text { Measure } 3 \text { - Develop an } \\
\text { entrepreneurial leadership }\end{array}$ & $\begin{array}{l}\text { "So the SSC will seek to break through (or round) the bureancracy in order to deliver good } \\
\text { service, and not to hide behind the bureaucracy as an excuse." [Member 1]. "It is important that } \\
\text { in developing entrepreneurial leadership that organization recognizes that there is structure to } \\
\text { entrepreneurialleadership. Often organizations mistake entrepreneurship with the removal of } \\
\text { all restrictions allowing each staff to act as if they "owned the business". This will lead to a } \\
\text { chaotic organization that is unsuccessfuL. "[Member 3]. }\end{array}$ \\
\hline $\begin{array}{l}\text { Measure } 4 \text { - Bring some people } \\
\text { from the industry to the public SSC }\end{array}$ & $\begin{array}{l}\text { "Bringing in a few individuals from the private sector to seed a public sector SSC with "best in } \\
\text { class" practices is a very effective strategy." [Member 3]. "There is alrearty a considerable } \\
\text { number of experienced professionals in best practices of SSC in the private sector in many } \\
\text { countries. Why not use their apprenticeship as a reference for the cultural change (role model) } \\
\text { in the public sector SSCs that need this change?" [Member 5]. }\end{array}$ \\
\hline $\begin{array}{l}\text { Measure } 5 \text { - Public SSC as a State } \\
\text { Policy }\end{array}$ & $\begin{array}{l}\text { 'Perfect, this is the most important issue for a Public SSC. The American norms should be applied } \\
\text { in other places where the politicians' meddling is intense, as Brazil" [Member 2]. "It is important } \\
\text { that the SSC have a clear mandate and that this mandate is isolated, as much as possible, from } \\
\text { the shifting and changing perspectives of politics. Establishing the SSC through the use of formal } \\
\text { legislative vehicles will protect, as much as is possible, from interference." [Member 3]. }\end{array}$ \\
\hline $\begin{array}{l}\text { Measure } 6 \text {-Develop organisational } \\
\text { resilience }\end{array}$ & $\begin{array}{l}\text { "I agree that, irrespective of the environment the SSC operates in it, should be able to adapt } \\
\text { quickly and respond to changing needs of its customers." [Member 3]. "It is about services and } \\
\text { ability to adapt to changing needs and stakeholder environments... if it can't do that it will fail just } \\
\text { like every other business on the planet." [Member 7]. }\end{array}$ \\
\hline
\end{tabular}

These six measures seek to improve the Public SSCs model, giving them alternatives to perfect a model, sometimes, only copied from the industry, without the necessary customisations (Ulbrich, 2010; Estabrooks et al., 2006), to consequently operate successfully in the public service.

(B) Quantitative part - In the first round, a comment caught my attention regarding those points on Market orientation and Excellence, apparently conflicting on their relationship with the SSC Performance, raised in the preliminary analysis. One of the ALS members commented that this is because the conceptual model has captured the current view of respondents and not the desired view for public SSCs. From this view, it is argued that public SSCs should, for sure, structure their service more and more from the customer focus. Thus, I proposed the first of two statements searching for ALS 
agreement (using a Likert scale), as follows. (1) It is desirable that the SSCs of the public sector are 'market-oriented' in their practices, that is, the design of its service operation considers the needs of its customers (besides other stakeholders) primarily. Note: 'market' understood here as the external environment to the SSC ('outside-in' view). It was also suggested by the ALS that the conceptual model (reflected in the survey questionnaire, Appendix B), could be transformed into part of a diagnosis tool for SSCs. This would help SSC managers to verify their degree of alignment with the best practices for this kind of service operation. Thus, I proposed the second of two statements searching for ALS agreement: (2) The conceptual model of this research (reflected in detail in the questionnaire), transformed into a diagnostic tool could be useful for an internal SSC assessment (managers and employees) and external (customers and stakeholders), providing a comparative analysis of perceptions, and resulting improvement actions.

The six ALS members, joining the second round, agreed with the two statements, and because of that decided to add two more measures to the first six measures proposed above: Measure 7 - Become 'market-oriented'; and Measure 8 - Using the conceptual model within a diagnostic framework, explained in Chapter 4. The following, I present some ALS members' comments on Measure 7.

ALS members' comments on Measure 7 - Member 3: "It is important that public sector SSC become Customer Service organisations that are "market-oriented," that is they understand their customer's challenges and the dynamics of the customers "business." It is also important that the SSC become a Shareholder-oriented organisation that is oriented to delivering the value which the organisation or individuals that formed the SSC hoped to achieve. It is the tension between these two stakeholder groups which causes 
organisations to be innovative and entrepreneurial." Member 6: "I agree that customer focus is key and important, but it's critical that federal SSCs also ensure they focus on compliance to rules, regulations, etc. and that could conflict with what the customers want now.” Member 7: “...your questions also imply an SSC is not a business... my SSC runs as if it were... it has over 2000 employees, E90m U0 and multiple clients (over 100 of varying sizes), yet entirely owned and run by three public sector shareholding organisations... to get to that scale, you have to run as though you are a business... focused on your shareholders, customers, have the right skills, respond to market conditions, etc..."

ALS members' comments on Measure 8 - Member 1: "Difficult to give a single answer to so many different types of SSC, from the expert advisory to the bulk transactional." Member 3: "I can see that the content of this research could assist SSC's in improving their business and in engaging with key stakeholders." Member 6: "The analysis you conducted identified key predictors of performance which would be the components that would be most helpful for SSCs to assess themselves.” Member 7: “...there ultimately has to be a real cultural buy in... I have spoken at many shared services conferences/workshops... I ask the audience how many are the full support of their MD/senior stakeholders if they're involved in the company project to set up an SSC... I then nicely say, because if they are not then they might leave now... creating any shared venture SSC or otherwise is entirely and Emotional not a Logical journey... after all, it is a marriage of different organisations."

At the end of the second round of the ALS discussions, the last revisions and adjustments resulting from interaction with the experts were consolidated, and the knowledge generated by the research was transformed into actionable knowledge (the eight measures). 
I would like to emphasise here, how I interpreted and understood the learning set discussion results to reach the recommendations. The purpose of the ALS work was not only to validate my preliminary findings but to bring the SSCs and the public-sector reality, doing a reality check. Thus, the conclusions drawn from the first round of discussion were again submitted to the ALS to be transformed into actions to improve the SSCs of the public sector. Therefore, my work in interpreting the results came both from the light of the literature and from the help of experienced practitioners of relevant SSCs.

To conclude my reflections, I would like to highlight some contradictions that I have found among the various findings. I will mention three examples, one illustrating differences between the findings of the quantitative and qualitative parts of the survey, and the other two are diverging views of ALS members. First example - One contradiction to note is that although the positive relationship between Leadership and Excellence has not been statistically confirmed in the conceptual model, the ALS understood that leadership is critical for the existence of excellence in an SSC. Second example - In the first round of discussions of the ALS, during the debate on the Category 01 Private Sector SSC as a service unit, all members agreed that the starting point of the design of an SSCs of the industry tends to be the perspective of the client (outside-in view), differently from what occurs in the public sector. The only exception was Member 3 , with the argument that both in the public sector, and in private sector, the starting point for the design are the objectives established for the SSC, not the client. Third example - During the discussion of Category 03 Private Sector SSC as a Business Unit, again, all ALS members agreed that public SSCs should be established as 'business units' (e.g. charging for services delivered, with a service culture oriented to the client). The 
only exception this time, was Member 4, arguing that public service should not follow the economic logic that drives private enterprise. According to him, this would go against the principles of public service, such as impersonality, participation, and transparency.

\subsection{Chapter Summary}

This chapter shows how the Action Research process applied in this thesis resulted in eight measures for properly adaptation and improvement of public SSCs. It also presents the quantitative and qualitative approaches conducted. The test of the conceptual model, on the quantitative side, and on the qualitative side by applying two open-ended questions to understand the existence of significant differences between the SSCs from the public and private sectors. This 'reading' of the contextual qualitative reality of public SSCs showed to be essential to a better use of the findings obtained by the quantitative modelling since it helped to understand and interpret the modelling results. Moreover, this chapter presents a brief account of my experience and personal growth as a researcher of the SSC theme, working in an Action Learning Set.

In the next chapter, the results of each stage of the research, presented in chapter three, are discussed, and conclusions and opportunities for SSC improvement structured, so that the public SSCs managers can benefit from new knowledge generated, and are also analysed the implications of this new knowledge. 
CHAPTER 4

CONCLUSIONS, IMPLICATIONS, AND RECOMMENDATIONS 


\subsection{Chapter Introduction}

The SSCs play a prominent role in the public-sector service operations, and increasingly governments are switching to this organisational model, managing to reduce billions of dollars in costs and improve the quality of public service. However, there are reports of failures and significant losses in unsuccessful civil SSC initiatives, with equally billion-dollar losses. This study proposes a model of factors that influence the performance of SSCs and eight measures for properly adaptation and improvement of public SSCs, which can help prevent failures both existing as well as new SSC to be deployed.

This chapter aims to discuss the results of the study, focusing on the proposed model and the eight measures mentioned for SSC adaptation and improvement, and is organised to match and complement the Chapter 3, that presents the results of the research, with four sections in addition to this: Research Aims Achievement, Discussion of the Results and Recommendations for Public SSC Managers, Conclusions, and Implications.

\subsection{Research Aims Achievement}

Next, I examine how each of the research objectives was achieved, to what extent this happened, and what it means, recapping and discussing each one. This discussion takes place here in a synthetic form, and further details are provided in the following section that discusses the results more extensively.

How the research objectives were achieved - Recalling, three objectives were established for this study (see Chapter) with a view (1) to analyse the factors that influence the performance of SSCs; (2) to investigate possible differences between 
public and private SSCs; and (3) to analyse potential evidence of inadequate copies of the SSC model of the industry by the public sector. All objectives were achieved, as discussed below. To operationalise the objective 1, it was elaborated the research question RQ1: To what extent does factors such as culture, leadership, resources and readiness for change, influence on service excellence, market orientation and performance of Shared Services Centres? This research question is related to the conceptual model proposed by this work (see Section 2.6), which has been tested and validated empirically in the quantitative action research part (Section 3.11) and had a positive relationship between the variables confirmed. The objective 2 was operationalised through RQ2: Do Shared Services Centres of the Public Sector differ from the SSCs of the Private Sector? This research question was answered affirmatively, because it was found in the qualitative part of the research (Section 3.12) that there are significant differences between public and private SSCs, particularly as for their operational contexts, and that the SSC model of the public sector must address these differences appropriately to be successful (eight proposed measures for adaptation and improvement of current public SSCs that need it are discussed in the next section). As for objective 3 , its operationalisation also occurred through the qualitative part of the research (Chapter 3) and was identified evidence of the existence of civil SSCs experiencing problems arising from using improperly copied models of the industry. There were reports of respondents and members of the Action Learning Set, which reinforced this conjecture. Other studies will be necessary to deepen the findings and analysis, opening opportunities for further research. 


\subsection{Discussion of the Results, Relevant Actions, and Recommendations for Public SSC Managers}

Public SSCs have played a key role in improving the public service and reducing operating costs in developed and developing countries, as part of government policies often. With this, governments can release billions of dollars that would be allocated to operational expenses and direct them to their core activities such as education, health, transportation, security, etc. However, there are accounts of failure and loss in the implementation of SSCs in the public sector. This study extends the knowledge of the SSCs of the public sector to propose and empirically test a model of factors that influence the performance of SSCs. In addition to substantiating the importance of each factor of the model, there are eight proposed measures, related to these factors, which public managers can take to improve their SSCs models, existing or to be implemented to help to prevent new cases of failure.

The proposed model represents the relationship of six factors (culture, leadership, resources, readiness for change, excellence, and market orientation) that affect performance (seventh factor) of an SSC and the results of empirical research that ratified the hypotheses of relationship between these factors are discussed below in the light of the results obtained. The only exception was for the relationship between leadership and excellence, whose evidence was not statistically proven, as discussed below. To recall, the established hypotheses were H1: Culture relates positively to Excellence in SSCs; H2: Leadership relates positively to Excellence in SSCs; H3: Resources relates positively to Excellence in SSCs; H4: The greater the Readiness for Change, the higher the level of the Excellence in SSCs; H5: Excellence relates positively to Market Orientation in SSCs; and H6: Market Orientation relates positively to Performance in SSCs. 
Culture - The results confirm the existence of a positive relationship between culture and excellence. Thus, the more the culture is, for example, oriented to quality and customer service, and engaged in the mission of the SSC, the higher the operational excellence. Other studies have also proven that the organisational culture is related to excellence (e.g. Oliveira \& Roth, 2012; Mohammad Qawasmeh et al., 2013; Irani et al., 2004; Oakland, 2001). Research findings suggest that culture is an enabler of service excellence and that it contributes to the success of the strategy desired with the deployment (for example, quality of service to citizens). The established culture in the public SSC is one of the determinants of success in providing quality services and the final performance of the SSC. One of the eight measurements discussed later, Measure 1, relates to these findings.

Leadership - The results showed no statistical significance and led to the rejection of the hypothesis that there is a positive relationship between leadership and excellence (see Section 3.11.4). This could suggest that the leadership style is not directly related to the achieved level of excellence, but it is not the case, as several other studies, for example, in the field of quality, confirmed the existence of this positive relationship (Prabhu \& Robson, 2000; Kanji, 1998; Mohammad Qawasmeh et al., 2013). With the information available, I could not find a conclusive reason for the lack of statistical significance; causes can be related to the sample and respondents, for instance. A final analysis of the reasons for this discrepancy will require further study, possibly in a new research. As the qualitative part of the research, in addition to studies of other researchers, corroborated strongly (including the Action Learning Set) the importance of leadership to an appropriate degree of excellence and performance of the SSC, and this factor led to an improvement measure as a suggestion of this study, see Measure 3. 
Resources - The results indicate that there is a positive relationship between resources and excellence. This is also a relation proven in numerous other studies as Barney (1991) and Fok-Yew \& Ahmad (2014). Another main finding is that the way the financial, human, and specific to services resources, among others, are organised and used is so important for excellence, as cost reduction or improving quality (Fok-Yew \& Ahmad, 2014). For this reason, the SSC must organise its resources in a vision and approach for a service operation. This finding suggests that the SSC in pursuit of excellence should also be aware of the differentiated orchestration of their resources, as specified in Measure 8, discussed later.

Readiness for change - The results show that the relationship between readiness for change and excellence is positive. This means that the higher the commitment of managers and employees with the desired organisational changes, and the better the SSC's competencies to lead change, the more favourable are the conditions to achieve excellence. Certainly, these competencies need to be incorporated in the SSC culture in greater or lesser degree, depending on the requirements of the portfolio of services offered. I.e. services that require more flexibility, and continuous changes will require skills and culture more favourable to change fastest than more stable services that require very few variations over the years (Goldstein et al., 2002; Clark et al., 2000). Even so, in the latter case, the customer service and the way services are provided (processes) will always need to be improved, and SSC employees be prepared to accept and adhere to the changes, preferably being co-responsible for the design and implementation of the changes (Kotter, 2001; Anderson \& Anderson, 2010).

Excellence - The results confirm the positive relationship between excellence and market orientation. Achieving service excellence means, in practice, exceeds the initial 
expectations of the customer (Gouthier et al., 2012; Johnston \& Clark, 2001). It also means providing quality service, be compared to the best in the category (benchmarking), have established quality standards and be oriented to delivering value to customers and other stakeholders (Camarero, 2007; Hietschold et al., 2014; Noble et al., 2002). For this, contributing factors as an organisational culture defined and implemented to promote excellence, and likewise, the leadership, resources, and readiness for change. The results from this study suggest that in the opinion among the survey respondents, excellence contributes directly with more emphasis on the SSC's performance than to market orientation. That is, the greater the degree of excellence, the better the performance, and with less intensity, the greater the market orientation (customers). As discussed in Chapter 3, the ALS experts believe that the trend is that the relationship between excellence and market orientation is strengthened as the SSCs reach new levels of maturity and customer focus ('outside -in' view).

Market orientation - The results indicate that the relationship between performance and market orientation is positive. Thus, to be able to deliver quality services, be proactive and have great performance, an SSC needs to be oriented to its clients and open to the external environment (Jaworski \& Kohli, 1993). In this way, it will be possible to meet customer expectations and comply with the other stakeholders. However, guiding the SSC to their clients is just the first step because usually, this first movement leads the organisation only to answer to what the customer requests, which is called responsive market orientation. Though, to have high-level performance, the SSC needs to go one step further and practice what is called proactive market orientation, which consists of anticipating the customers' latent needs (Narver et al., 2004). The absence of competition in the public service tends to lead the SSCs not to achieve optimal 
performance. In this context, market orientation with its management practices and mechanisms can compensate for the lack of competition, and help the SSC managers in the continuous improvement of the service operation.

Performance - The results show that the performance is influenced by market orientation, as per excellence. The empirical validation of the model has shown that the relationship between performance and excellence is more intense than between market orientation and performance (as discussed in Section 3.11 Quantitative Action Research) when it was expecting the opposite. These findings, in the interpretation of the Action Learning Set, is because the model has captured the view of the current situation of the SSCs and not the desired situation, or in future with a higher level of maturity. Performance here is not limited to the financial dimension, but is extended, for example, the positive impact that the SSC has in society, motivation and productivity of their employees, resources dedicated to monitoring performance, customer satisfaction, and coordinated implementation of strategy defined for the SSC (see Section 2.6 Conceptual Model).

In short, culture, leadership, resources, readiness for change, excellence, and market orientation, are key predictors of performance, and this means that if these factors are organised, developed and operated properly, and achieve high levels of best practices, will influence decisively, so there is high performance of the SSC. The findings from the quantitative and qualitative results (e.g. Table 3.27), were discussed on the Action Learning Set composed of experts in SSC and public service (see Chapter 3). The experts gave suggestions and commented on the results and preliminary findings I found. Based on these insights, I developed the first version of the eight measures for proper adaptation and improvement of civil SSCs that were then submitted to the 
ALS for refinement, resulting in these eight measures discussed here. The eight measures summarise the knowledge generated from my research, and facilitate the operationalisation of the research knowledge by the public SSCs managers. The following I present and discuss each one of these measures:

Measure 1 - Create its own service culture. It has been found by the qualitative research that many public SSCs lack adequate culture for service oriented to the customer (customer centric culture) and the lack of resources for investment makes them less competitive than the private SSCs. It should be allowed for an SSC have its own culture, oriented to the delivery of service to the customer/citizen with high employee engagement; combining with creative responses, the excellence in service with the necessity of fulfilment of the legislation and the limitations inherent in the civil service. This culture must also be appropriate and aligned with the culture of the client organisations served. The SSC should also have mechanisms of 'chargeback' (Bergeron, 2002), charging for their services and thus having the resources to invest, to maintain high levels of excellence in service. While paying for the service, the clients also will be compelled to be more demanding, taking the SSC to a virtuous cycle of continuous improvement. In services provided directly to citizens, the SSC may charge, for instance, the organs of core activities, since the citizen already pay his taxes. Care should be taken that the SSC compensation model does not take to limit their focus to acting only in 'their' processes, but also to integrate 'its processes' to the processes of its customers (actually processes should be (re)designed from end to end). Cultural adaptation also needs to be made to the client organisations to assimilate the 'chargeback,' so don't try to avoid the service because it is charged. However, beyond that, not to generate resistance in the SSC clients, the collection of chargeback must be made with due care, 
so that they feel that the value and form of the collection are fair. If not in this way, the chargeback can derail the success of the SSC. The moments of the greatest need of austerity tend to favour the adoption of chargeback, and the decision as to the form of the collecting, values by type of service and the SSC margin, must pass the approval of the Board of customers in a forum discussed in the Measure 2 the following. Below, Table 4.1 presents the relevant actions to developing and implementing the Measure 1, which I suggest to the public SSC managers.

Table 4.1 Relevant actions to take the Measure 1 - Create its own service culture

\begin{tabular}{|c|c|}
\hline RELEVANT ACTIONS & $\begin{array}{l}\text { Factors influencing the SSC } \\
\text { performance impacted by these } \\
\text { relevant actions (Section } 2.6 \text { ) }\end{array}$ \\
\hline \multirow{3}{*}{$\begin{array}{l}\text { 1.Carry out a diagnosis of the SSC culture of service to check } \\
\text { the existent gaps between current and desired culture. }\end{array}$} & \begin{tabular}{l|l}
$\mathrm{X}$ & Culture \\
\end{tabular} \\
\hline & \begin{tabular}{l|l}
$\mathrm{X}$ & Leadership \\
\end{tabular} \\
\hline & \begin{tabular}{l|l|}
$\mathrm{X}$ & Resources \\
\end{tabular} \\
\hline \multirow{2}{*}{$\begin{array}{l}\text { 2.Conduct conceptual alignment about the culture of } \\
\text { service with the whole management. }\end{array}$} & \begin{tabular}{l|l}
$\mathrm{X}$ & Readiness for change \\
\end{tabular} \\
\hline & \begin{tabular}{l|l|}
$\mathrm{X}$ & Excellence \\
\end{tabular} \\
\hline \multirow{2}{*}{$\begin{array}{l}\text { 3.Develop and implement a communication and awareness } \\
\text { plan (internal and external) about the culture of service. }\end{array}$} & \begin{tabular}{l|l}
$\mathrm{X}$ & Market orientation \\
\end{tabular} \\
\hline & \begin{tabular}{l|l}
$\mathrm{X}$ & Performance \\
\end{tabular} \\
\hline \multicolumn{2}{|l|}{$\begin{array}{l}\text { 4.Apply the diagnostic framework 'Factors for design and } \\
\text { analysis of service operations' to identify which service } \\
\text { attributes need to be improved (Section } 3.12 .2 \text { ). }\end{array}$} \\
\hline \multicolumn{2}{|l|}{ 5.Conduct training at all levels of the SSC. } \\
\hline \multicolumn{2}{|l|}{$\begin{array}{l}\text { 6.Adjust the other mechanisms and systems of } \\
\text { management (e.g. performance, compensation) to reflect } \\
\text { the attitude of the people regarding the culture of service. }\end{array}$} \\
\hline \multirow[t]{2}{*}{ See also the following section(s): Section 2.6 and 3.4} & \\
\hline & $\begin{array}{l}\text { Note: ' } X \text { ' means that the factor is impacted } \\
\text { by the relevant actions mentioned }\end{array}$ \\
\hline
\end{tabular}

Measure 2 - Steering by its customers (client focus). The research revealed that many public sector SSCs operate with a vision toward inside (inside-out), when they should run from the customer focus (outside-in), and that because of it have impaired performance and dissatisfied customers. An SSC must, by definition, be directed by its customers, and this direction level is typically more strategic (not in the daily management of the SSC), through a Council or similar mechanism. In this strategic 
board, the customer should have a voice and the power of veto in any decision relevant to the life of the SSC, and not only in matters of his specific interest. Moreover, the SSC should not be seen only as a form of cost reduction, but also a provider of best services to its customers, corresponding to their quality expectations. The public SSC will naturally be 'more competitive' if guided and directed by its customers (in the words of one of the members of the ALS to 'Do what the customer needs'). Note: the meaning of 'more competitive' here is to respond with effectiveness to the needs of the SSC customers. On the other hand, it is essential that the SSC learns to balance what the client requests with the need for standardisation and economies of scale, which will bring a significant part of the cost reduction and the final value for the existence of SSC. Another important aspect is that depending on the nature of the service provided by the SSC client may be an internal organ or the citizen. Therefore, it is necessary to define who is the customer who will be part of the board (e.g. the owners of the SSC, the stakeholders, those who pay the SSC for the services rendered?). And if it is not the end customer, they should also be heard through other mechanisms - that should always be part of all SSC-as, for example, periodic satisfaction surveys (e.g. after each customer service via phone, sent by email or placed to the client access portals, as well as research with probabilistic sampling), complaint channels that work in a structured way (e.g. web portals), ombudsman channel, etc. Below, Table 4.2 presents the relevant actions to developing and implementing the Measure 2, which I suggest to the public SSC managers. 
Table 4.2 Relevant actions to take the Measure 2 - Steering by its customers (client focus)

\begin{tabular}{|c|c|}
\hline RELEVANT ACTIONS & $\begin{array}{l}\text { Factors influencing the SSC } \\
\text { performance impacted by these } \\
\text { relevant actions (Section } 2.6 \text { ) }\end{array}$ \\
\hline \multirow{3}{*}{$\begin{array}{l}\text { 1.Define and deploy the Council of customers and other } \\
\text { stakeholders, to decide on the strategic issues of the SSC. }\end{array}$} & \begin{tabular}{|l|l|}
$\mathrm{X}$ & Culture \\
\end{tabular} \\
\hline & \begin{tabular}{|l|l|}
$\mathrm{X}$ & Leadership \\
\end{tabular} \\
\hline & \begin{tabular}{|l|l|}
$\mathrm{X}$ & Resources \\
\end{tabular} \\
\hline \multirow{2}{*}{$\begin{array}{l}\text { 2. Implement periodic research and other mechanisms to } \\
\text { check continuously customer satisfaction. }\end{array}$} & \begin{tabular}{|l|l|}
$\mathrm{X}$ & Readiness for change \\
\end{tabular} \\
\hline & \begin{tabular}{|l|l|}
$\mathrm{X}$ & Excellence \\
\end{tabular} \\
\hline \multirow{2}{*}{$\begin{array}{l}\text { 3.Deploy direct channel for complaints and suggestions } \\
\text { from customers. }\end{array}$} & \begin{tabular}{|l|l|}
$\mathrm{X}$ & Market orientation \\
\end{tabular} \\
\hline & \begin{tabular}{|l|l|}
$\mathrm{X}$ & Performance \\
\end{tabular} \\
\hline \multirow[t]{2}{*}{ See also the following section(s): Section 2.6} & \\
\hline & $\begin{array}{l}\text { Note: ' } \mathrm{X} \text { ' means that the factor is impacted } \\
\text { by the relevant actions mentioned }\end{array}$ \\
\hline
\end{tabular}

Measure 3 - Develop an entrepreneurial leadership ${ }^{1}$. The results of the inquiry point that, though many public SSCs are high-performance operations of services, comparable to the best of the industry, others are driven by administrators and not leaders, with performance complacency and more preoccupied in paying attention to the political context than to the clients of the SSC. An entrepreneurial leadership (Osborne, 2006, 2010; Keuper \& Lueg, 2013), as part of an entrepreneurial culture (see Section 2.2.2), can revolutionise a lenient, too indulgent SSC, even with the limitations arising from bureaucracy, scope defined by law, politics, and limitations related to staff. This can lead to the transformation of the attitude of the SSC staff and positively affect service quality. As discussed in Section 2.6.4, an effective leader must have strong skills to drive change towards continuous improvement of a public SSC, inspiring staff with their conduct in defence of service excellence in every decision made daily. This leadership should not be a 'transactional leadership,' oriented to everyday transactions, but a 'transformational leadership,' as argued by Kanji \& Sá (2001), that every day leads to a continuous transformation of the public SSC towards excellence in service. With such leadership, the SSC will seek to provide a public service with excellence, despite 
the bureaucracy of the public sector. On the other hand, a challenge always present will be to find the right leader for the SSC context in question, as well as having an entrepreneurial attitude, and lead and motivate staff towards excellence in service. This leader should have other equally important leadership predicates, not discussed here, but, which will need to be described in the profile of the desired head when designing the SSC model. This measure will also help reduce the impact of problems that were reported by survey respondents as the high turnover of staff in many public SSCs and lack of leadership control over who will work in their teams. Below, Table 4.3 presents the relevant actions to developing and implementing the Measure 3, which I suggest to the public SSC managers.

Table 4.3 Relevant actions to take the Measure 3 - Develop an entrepreneurial leadership

\begin{tabular}{|c|c|}
\hline RELEVANT ACTIONS & $\begin{array}{l}\text { Factors influencing the SSC } \\
\text { performance impacted by these } \\
\text { relevant actions (Section } 2.6 \text { ) }\end{array}$ \\
\hline \multirow{4}{*}{$\begin{array}{l}\text { 1.Set the desired profile for entrepreneurial leadership (top } \\
\text { and middle management), after enough discussion of the } \\
\text { subject with the SSC managers, and the other } \\
\text { stakeholders. }\end{array}$} & \begin{tabular}{l|l}
$\mathrm{X}$ & Culture \\
\end{tabular} \\
\hline & \begin{tabular}{l|l}
$\mathrm{X}$ & Leadership \\
\end{tabular} \\
\hline & Resources \\
\hline & \begin{tabular}{l|l}
$\mathrm{X}$ & Readiness for change \\
\end{tabular} \\
\hline \multirow{3}{*}{$\begin{array}{l}\text { 2.Align the profile of entrepreneurial leadership to the } \\
\text { other management mechanisms, such as performance and } \\
\text { compensation. }\end{array}$} & \begin{tabular}{l|l}
$\mathrm{X}$ & Excellence \\
\end{tabular} \\
\hline & \begin{tabular}{l|l}
$\mathrm{X}$ & Market orientation \\
\end{tabular} \\
\hline & \begin{tabular}{l|l|}
$\mathrm{X}$ & Performance \\
\end{tabular} \\
\hline \multicolumn{2}{|l|}{$\begin{array}{l}\text { 3.Train managers and staff, and deploy the new profile of } \\
\text { entrepreneurial leadership. }\end{array}$} \\
\hline \multirow[t]{2}{*}{ See also the following section(s): Section 2.6} & \\
\hline & $\begin{array}{l}\text { Note: ' } X \text { ' means that the factor is impacted } \\
\text { by the relevant actions mentioned }\end{array}$ \\
\hline
\end{tabular}

${ }^{1}$ Note: Despite the hypothesis H2 (Leadership relates positively to excellence) was rejected (see Section 3.11.6), the Action Learning Set considered leadership as a critical element to lead the civil SSC to be more client-oriented.

Measure 4 - Bring some people from the industry to the public SSC. It has been found in the literature review, and corroborated by the results of the research that, 
although in some countries the level of best practices in public service SSCs is comparable to those of the industry, many public servants working in other SSCs have limitations in their training, experience, or mainly, attitude. Thus, people with the right skills to promote high-performance SSCs could be brought from the industry to the SSCs of the public sector that needs them. This could occur (with the appropriate legal mechanisms in place) for instance on a temporary (but, not too short) basis, by project, or other similar initiatives. Public-private partnerships could also provide the legal means for the establishment of SSCs that can count on professionals from the industry; they can disseminate best practice and form a culture that favours acting as a clientoriented service unit. A member of the ALS commented that the public sector would need to develop mechanisms to identify the right people for doing that. Another challenge in some countries may be to convince these professionals to go to the publicsector due to substantial differences in the characteristics and dynamics inherent to each sector workplace. Below, Table 4.4 presents the relevant actions to developing and implementing the Measure 4, which I suggest to the public SSC managers.

Table 4.4 Relevant actions to take the Measure 4 - Bring some people from the industry to the public SSC

\begin{tabular}{|c|c|}
\hline RELEVANT ACTIONS & $\begin{array}{l}\text { Factors influencing the SSC } \\
\text { performance impacted by these } \\
\text { relevant actions (Section } 2.6 \text { ) }\end{array}$ \\
\hline \multirow{3}{*}{$\begin{array}{l}\text { 1.Identify whether Measure } 4 \text { can help make your SSC more } \\
\text { customer-oriented, considering its specific context. }\end{array}$} & \begin{tabular}{l|l|}
$\mathrm{X}$ & Culture \\
\end{tabular} \\
\hline & \begin{tabular}{l|l}
$\mathrm{X}$ & Leadership \\
\end{tabular} \\
\hline & \begin{tabular}{l|l|}
$\mathrm{X}$ & Resources \\
\end{tabular} \\
\hline \multirow{4}{*}{$\begin{array}{l}\text { 2.Develop/ Adopt legal mechanisms, internal skills and } \\
\text { conditions to attract industry professionals with the } \\
\text { appropriate profile, to act as catalysts for the new service } \\
\text { culture. }\end{array}$} & \begin{tabular}{l|l}
$\mathrm{X}$ & Readiness for change \\
\end{tabular} \\
\hline & \begin{tabular}{l|l}
$\mathrm{X}$ & Excellence \\
\end{tabular} \\
\hline & \begin{tabular}{l|l}
$\mathrm{X}$ & Market orientation \\
\end{tabular} \\
\hline & \begin{tabular}{l|l}
$\mathrm{X}$ & Performance \\
\end{tabular} \\
\hline 3.Implement and evaluate the contribution of the initiative. & \\
\hline \multirow[t]{2}{*}{ See also the following section(s): Section 2.6} & \\
\hline & $\begin{array}{l}\text { Note: ' } \mathrm{X} \text { ' means that the factor is impacted } \\
\text { by the relevant actions mentioned }\end{array}$ \\
\hline
\end{tabular}


Measure 5 - Public SSC as a State Policy. Another finding of the research is that many SSCs are susceptible to political interference affecting their performance and customer service quality. This is especially serious if we consider the fact that more and more countries should now adopt the SSCs as a solution for reducing operating costs and improving public service quality, as recommended by OECD $(1995,2010)$. The SSC should be protected by law (or equivalent) against political interference (whether at the federal, state or regional/municipal level), not to be a management practice dependent on an elected Government-which will govern during a relatively short period of timeto avoid being subsequently discontinued, or severely impacted, when there is a change of Government. In the words of one member of the ALS "It is important that the SSC has a clear mandate and that this mandate is isolated, as much as possible, from the shifting and changing perspectives of politics. Establishing the SSC using formal legislative vehicles will protect, as much as is possible, from interference." Another ALS member informs that in the US, for example, the level of this kind of political interference (in federal SSCs), practically does not exist. This is not a matter of wanting to force the adoption of SSC by law, as it most likely would not work, but once defined by consensus that SSC is an appropriate solution, to protect services operation and the results for citizens of vested political interests (political shadowy interests/hidden motives), especially in countries where the practices and mechanisms of democracy are less perfected. Below, Table 4.5 presents the relevant actions to developing and implementing the Measure 5, which I suggest to the public SSC managers. 
Table 4.5 Relevant actions to take the Measure 5 - Public SSC as a State Policy

\begin{tabular}{|c|c|}
\hline RELEVANT ACTIONS & $\begin{array}{l}\text { Factors influencing the SSC } \\
\text { performance impacted by these } \\
\text { relevant actions (Section } 2.6 \text { ) }\end{array}$ \\
\hline \multirow{7}{*}{$\begin{array}{l}\text { 1.Analyse the risk and consequence of undue political } \\
\text { interference in the SSC. } \\
\text { 2.Set a clear mandate for the SSC and assured continuity. } \\
\text { 3.Protect the SSC from undue political interference via } \\
\text { politics and legislative vehicles. }\end{array}$} & Culture \\
\hline & Leadership \\
\hline & Resources \\
\hline & Readiness for change \\
\hline & Excellence \\
\hline & Market orientation \\
\hline & \begin{tabular}{l|l}
$\mathrm{X}$ & Performance \\
\end{tabular} \\
\hline \multirow[t]{2}{*}{ See also the following section(s): Section 2.6} & \\
\hline & $\begin{array}{l}\text { Note: ' } \mathrm{X} \text { ' means that the factor is impacted } \\
\text { by the relevant actions mentioned }\end{array}$ \\
\hline
\end{tabular}

Measure 6-Develop organisational resilience. The context of this measure may, to some extent, be benefited by Measure 5, because if there is specific legislation to 'shield' the SSC against undue political interference, this may also contribute to making a public SSC resilient (Brunsdon \& Dalziell, 2005). But, in addition, public SSCs should have the ability to adapt quickly, respond and continue to provide high-quality services to its customers, regardless of possible changes brought about in the political context. (Shield the SSC against any kind of interference.) My research found that the political aspects negatively affect several SSCs in the public sector. It also noted that the greater number of stakeholders in these SSCs affects its ability to provide quality services to its customers. These findings highlight the need for greater public SSC resilience. Can contribute to this resiliency: The financial independence of public SSC, with the adoption of chargeback (discussed in Measure 1); governance instruments such as the board with the effective participation of clients (see Measure 2); and likewise, the clear SSC's mission definition for which can be driven as an 'independent' service unit, and therefore, also less susceptible to organisational interference. Here too, the adequacy of the relevant legislation is an important aspect, and should even cover the human factor, 
to have lower turnover and to choose professionals with the right profile to compose SSC teams. Below, Table 4.6 presents the relevant actions to developing and implementing the Measure 6, which I suggest to the public SSC managers.

Table 4.6 Relevant actions to take the Measure 6 - Develop organisational resilience

\begin{tabular}{|c|c|}
\hline RELEVANT ACTIONS & $\begin{array}{l}\text { Factors influencing the SSC } \\
\text { performance impacted by these } \\
\text { relevant actions (Section } 2.6 \text { ) }\end{array}$ \\
\hline \multirow{3}{*}{$\begin{array}{l}\text { 1. Keep risk management mechanisms continuously } \\
\text { monitoring the political and regulatory environment in } \\
\text { which the SSC is inserted (Brunsdon \& Dalziell, 2005). }\end{array}$} & \begin{tabular}{l|l}
$\mathrm{X}$ & Culture \\
\end{tabular} \\
\hline & \begin{tabular}{l|l}
$\mathrm{X}$ & Lead \\
\end{tabular} \\
\hline & \begin{tabular}{l|l}
$\mathrm{X}$ & Reso \\
\end{tabular} \\
\hline \multirow{3}{*}{$\begin{array}{l}\text { 2.Develop a business continuity plan (Brunsdon \& Dalziell, } \\
\text { 2005). }\end{array}$} & \begin{tabular}{l|l}
$\mathrm{X}$ & Readi \\
\end{tabular} \\
\hline & \begin{tabular}{l|l}
$\mathrm{X}$ & Excellence \\
\end{tabular} \\
\hline & \begin{tabular}{l|l}
$\mathrm{X}$ & Market orienta \\
\end{tabular} \\
\hline $\begin{array}{l}\text { 3.Ensure to have capacity for rapid SSC adaptation to the } \\
\text { new external realities, for example, keeping the SSC with } \\
\text { high level of readiness for change. }\end{array}$ & \begin{tabular}{l|l}
$\mathrm{X}$ & Performance \\
\end{tabular} \\
\hline \multicolumn{2}{|l|}{$\begin{array}{l}\text { 4.Mastering the ability to change management to reduce } \\
\text { the time of response to external demands (see 2.6.4). }\end{array}$} \\
\hline \multirow{2}{*}{$\begin{array}{l}\text { See also the following section(s): Section } 2.6 \\
\text { Further insights to Measure } 6 \text { deployment on Brand (2006) }\end{array}$} & \\
\hline & $\begin{array}{l}\text { Note: ' } X \text { ' means that the factor is impacted } \\
\text { by the relevant actions mentioned }\end{array}$ \\
\hline
\end{tabular}

Measure 7 - Become 'market-oriented.' As discussed in Section 2.6.4., the public SSCs in countries like the USA and the UK were born under the mantle of New Public Management, which encourages the public service to be market-oriented. However, this research showed that many civil SSCs, in those and other countries, do not have the best practices in services and performance comparable to the private SSCs because they are not oriented to their customers. It is desirable (and practically compulsory to be a truthful SSC) that the SSCs of the public sector are 'market-oriented' in their practices, that is, the design of its service operation considers primarily the needs of its customers (in addition to other stakeholders). A better understanding of customer needs can make the SSC most efficient in providing services (Jaworski \& Kohli, 1993), as well as the knowledge of the challenges and dynamics of the clients 'business'. Moreover, with MO 
is also easier to identify and meet the latent needs of customers. Note: 'market' is understood here as the external environment to the SSC ('outside-in' view). Important to note that the service design should consider the level of flexibility possible, because the SSC must, by definition, provide a high degree of standardisation in their processes, that is, limited flexibility, see Johnston (2004) and Frei (2006), to achieve its proposals to reduce costs and increase quality of service. It is also vital that the public SSC meets all legislation, and that it is 'shareholder-oriented,' that reaches its customers, but without losing the expectations of their 'proprietary' organisations. The tension caused by these stakeholder groups may result in an appropriate balance to a high-performance organisation. Below, Table 4.7 presents the relevant actions to developing and implementing the Measure 7, which I suggest to the public SSC managers. 
Table 4.7 Relevant actions to take the Measure 7 - Become 'market-oriented'

\begin{tabular}{|c|c|}
\hline RELEVANT ACTIONS & $\begin{array}{l}\text { Factors influencing the SSC } \\
\text { performance impacted by these } \\
\text { relevant actions (Section 2.6) }\end{array}$ \\
\hline \multirow{4}{*}{$\begin{array}{l}\text { 1.Fine-tune the organisational factors culture, leadership, } \\
\text { resources, readiness for change, and excellence to become } \\
\text { market-oriented. }\end{array}$} & \begin{tabular}{l|l|}
$\mathrm{X}$ & Culture \\
\end{tabular} \\
\hline & \begin{tabular}{l|l}
$\mathrm{X}$ & Leadership \\
\end{tabular} \\
\hline & \begin{tabular}{l|l|}
$\mathrm{X}$ & Resources \\
\end{tabular} \\
\hline & \begin{tabular}{l|l}
$\mathrm{X}$ & Readiness for change \\
\end{tabular} \\
\hline \multirow{3}{*}{$\begin{array}{l}\text { 2.Adopt the Council of customers and other stakeholders } \\
\text { (see Measure 2), to be strategically oriented by your } \\
\text { clients. }\end{array}$} & \begin{tabular}{l|l|}
$\mathrm{X}$ & Excellence \\
\end{tabular} \\
\hline & \begin{tabular}{l|l|}
$\mathrm{X}$ & Market orientation \\
\end{tabular} \\
\hline & \begin{tabular}{l|l|}
$\mathrm{X}$ & Performance \\
\end{tabular} \\
\hline \multirow[t]{2}{*}{ See also the following section(s): Section 2.6} & \\
\hline & $\begin{array}{l}\text { Note: ' } X \text { ' means that the factor is impacted } \\
\text { by the relevant actions mentioned }\end{array}$ \\
\hline
\end{tabular}

Measure 8 - Using the conceptual model within a diagnostic framework. The performed literature review identified the lack of a diagnostic framework that can help public sector SSCs on their journey to become market-oriented. The Action Learning Set understood that the survey instrument used in this study (Appendix B) could be transformed into a diagnostic questionnaire and employed by the managers of public SSCs to identify their current state and establish their improvement goals. The diagnostic questionnaire could be useful for an internal SSC assessment (managers and employees) and external (customers and stakeholders), providing a comparative analysis of perceptions, and resulting improvement actions. From a methodological point of view, any adaptation to the questionnaire used in this research should be done in light of the theory discussed in the formulation of each of its seven constructs (Culture, Leadership, Resources, Readiness for change, Excellence, Market Orientation, and Performance), as detailed in Section 2.6 Conceptual Model. The first six constructs are performance predictors; thus, based on the proposed model, tested empirically, for the public SSC performance to be top, all these elements represented by the constructs must be effectively implemented. Below, Table 4.8 presents the relevant actions to 
developing and implementing the Measure 8, which I suggest to the public SSC managers.

Table 4.8 Relevant actions to take the Measure 8 - Using the conceptual model within a diagnostic framework

\begin{tabular}{|c|c|}
\hline RELEVANT ACTIONS & $\begin{array}{l}\text { Factors influencing the SSC } \\
\text { performance impacted by these } \\
\text { relevant actions (Section } 2.6 \text { ) }\end{array}$ \\
\hline \multirow{3}{*}{$\begin{array}{l}\text { 1.Develop a diagnostic tool for the SSC from the conceptual } \\
\text { model and questionnaire applied in this research }\end{array}$} & \begin{tabular}{l|l}
$\mathrm{X}$ & Culture \\
\end{tabular} \\
\hline & \begin{tabular}{l|l}
$\mathrm{X}$ & Leadership \\
\end{tabular} \\
\hline & \begin{tabular}{l|l}
$\mathrm{X}$ & Resources \\
\end{tabular} \\
\hline \multirow{2}{*}{$\begin{array}{l}\text { 2. Obtain the diagnosis of market orientation level of your } \\
\text { SSC. }\end{array}$} & \begin{tabular}{l|l}
$\mathrm{X}$ & Readiness for change \\
\end{tabular} \\
\hline & \begin{tabular}{l|l}
$\mathrm{X}$ & Excellence \\
\end{tabular} \\
\hline \multirow{2}{*}{$\begin{array}{l}\text { 3.Set goals about the degree of market orientation desired. } \\
\text { 4.Prepare and deploy an action plan to achieve the level of } \\
\text { market orientation set. }\end{array}$} & \begin{tabular}{l|l}
$\mathrm{X}$ & Market orientation \\
\end{tabular} \\
\hline & \begin{tabular}{l|l}
$\mathrm{X}$ & Performance \\
\end{tabular} \\
\hline \multirow{2}{*}{$\begin{array}{l}\text { See also the following section(s): Section } 3.8 \\
\text { See Section } 4.3\end{array}$} & \\
\hline & $\begin{array}{l}\text { Note: ' } \mathrm{X} \text { ' means that the factor is impacted } \\
\text { by the relevant actions mentioned }\end{array}$ \\
\hline
\end{tabular}

\subsubsection{Recommendations for Public SSC Managers}

As research products, the eight measures suggested above are generic, and their adoption and implementation require proper SSC candidate context analysis, which can start, for example, with the application of the survey instrument used as a diagnostic questionnaire (Measure 8). This will provide a first reading of how market-oriented the rated public SSC is, in its current situation. It will also allow identifying the existing gap in each construct, or dimension of analysis, and together with a feasibility study on the adoption of other measures, will allow establishing an action plan (for each dimension of the questionnaire/model) to raise the SSC to the category of market-oriented. The eight measures proposed can be implemented individually but would be more appropriate if considered and evaluated together. However, it is important to understand that an organisational transformation of this magnitude is quite complex and requires specific competences to conduct such a program successfully. Among these competences, I highlight the change management, discussed in section 2.6.4. Moreover, 
an organisational transformation program in a public SSC may be an appropriate context for using the methodological Action Research approach (see Chapter 3). In my view, the main advantage of using AR in this context is that it is an approach originally oriented to problem-solving (Coghlan \& Brannick, 2014). It provides a very practical approach, generating beyond the solution of the problems in scope, learning for all participants, in a systematic and democratic way.

\subsection{Conclusions}

It is common knowledge among managers and Shared Service Centre researchers that the success or failure of an SSC is related to factors that influence their performance. However, these factors need to be adapted to the SSC context, whether private or public. This study, pioneer in the exploration of the factors that influence the performance of SSCs, with emphasis on the public sector, brings three main original contributions to expand the body of knowledge in the promising field of SSCs. First, it provides a conceptual model, designed and tested empirically in this research, establishing the relationship between the factors: culture, leadership, resources, readiness for change, excellence, and market orientation, and the performance of an SSC. This enables managers to perfect their service operations, adjusting each factor to contribute accordingly to the performance of their respective SSC. For example, the study showed that the greater the degree of public SSC guidance to their customers (market orientation), the better their performance. Second, my empirical research has identified significant differences in the contexts of public and private SSCs. Therefore, this study suggests eight measures, discussed with experts in public sector and SSC, to adapt (or fine-tune) and improve public SSCs model to reflect the peculiarities of the public sector and are not simply a copy (not translated properly) of SSC models from the industry, 
because the industry has different dynamics and socio-economic purpose. These measures for public SSC are: creating an outside-in service culture, specific for the SSC; the SSC's customers actively participate in its strategic management; the establishment of an entrepreneurial leadership in SSC, leading employees to excellence in service; bring to the SSC public professionals from industry, experts in high-performance SSC; have adequate legislation to ensure that the SSC has a very low degree of negative political interference; develop organisational resilience to 'shield' the SSC against political manipulation; make the SSC customer-oriented (market oriented); and use the proposed conceptual model and the questionnaire on a diagnostic framework to adjust each factor and raise the SSC performance. Third, a contribution to the rescue of the history of the emergence of SSCs, because during the literature review, it was found that the roots of the SSC date back, at least, to the 1960s, in the US education sector, with an initiative called 'Rural Shared Service,' which can be considered an embryo of the current SSCs.

\subsection{Implications}

Although the findings of this study were obtained by following the scientific methodological rigor for quantitative and qualitative research, they are not intended only for academic scholars. They are proposed mainly for practitioners of Shared Services Centres and, therefore, are presented through suggestions of practical measures that can be used by public managers in improving the focus and the performance of current or future SSCs. Next, the implications of the results of this study are highlighted considering two audiences, public SSC managers and those responsible for public policy development. 


\subsubsection{Public SSC Managers}

The results of the proposed conceptual model validation confirmed the importance of the factors: culture, leadership, resources, readiness for change, excellence, and market orientation for the performance of public SSCs. Therefore, SSC managers in the public sector need to be aware of these factors and adjust them to the extent of the need for each SSC to achieve high levels of performance. To help to know what the level of need is, the questionnaire of this study can be applied within a diagnostic framework. It is recommended that this survey instrument is applied to respondents internal and external to the SSC. Internal respondents could be, for example, three groups: the senior managers, other managers, and staff in general; it will serve to capture existing perceived differences among these groups. The external respondents could be, for instance, the other stakeholders including the SSC customers' agencies. The results will show the current level of match of the SSC to the expectations of different groups participating in the survey, for each dimension of analysis (culture, leadership, resources, readiness for change, excellence, and market orientation). The results also will make possible to prepare a plan of action, and a gradual implementation strategy toward excellence in service, oriented by the customer focus (marketoriented).

Furthermore, it is recommended that the SSC managers of public service assess the applicability of each of the eight suggested measures, mentioned in the previous section and discussed in more detail in Section 5.4, adopting those that are relevant to their reality. The eight measures are intended to adapt the SSC model, that already exists or to be deployed whose starting model is usually inherited from the industry, without 
a proper translation (Estabrooks et al., 2006; Armstrong et al., 2013; Straus et al., 2009; Ulbrich, 2010) to the context of the public sector.

\subsubsection{Public Policy Makers}

To those who have the responsibility to establish the conditions for the provision of public service with high quality and low cost, in this case through the Shared Services Centre, the suggestion is to analyse the Measure 5 - Public SSC as a State Policy, proposed by this study. The research identified that one of the most severe problems faced by some SSCs, especially in contexts where the mechanisms of democracy are partially developed, is the political interference contrary to the SSC initiatives, for example, by ideology or just to discontinue or hinder the initiatives of competing political parties. The existence of legal support to 'shield' the governments SSCs against this type of political interference, can help in such circumstances and ensure that public resources are better used. This measure becomes even more important as there is a tendency to expand the adoption of SSCs by the public sector, especially in developing countries (OECD, 1995, 2010). The implications and opportunities for the community of researchers at the SSC theme are discussed in the next chapter. 
CHAPTER 5

REFLECTION AND LEARNING AS AN ACTION RESEARCHER 


\subsection{Chapter Introduction}

According to Coghlan \& Brannick (2014), one of the most significant results of a project that uses the Action Research (AR) approach is the learning and development of the action researcher. After several months of work on the project, and interaction with stakeholders, I can share here my learning, not only concerning methodology, or the subject under discussion, but also what has changed in my attitudes, assumptions, and skills as a scholar-practitioner. Since I opted not to use the 'reflective boxes' (Coghlan \& Brannick, 2014) in the previous sections to narrate my dilemmas and progress as an action researcher, I concentrate in this section all the most important reflections. I preferred to use a narrative style, because narration allows me to talk about my experience as a subject of the research, with the possibility of exploring several interpretations, and still dialoguing with the reader (Ramsey, 2014). I organised my reflections in two blocks. In the first block, 5.2 Reflections on the Entire Research Project, I discuss the aspects common to all the steps of my research project. In the second block, 5.3 Reflections on Each Step of the Research Project, I address the issues associated with each step of the project, following the learning roadmap proposed by Coghlan \& Brannick (2014), which shows the main steps covered in the development of a doctoral thesis applying Action Research.

\subsection{Reflections on the Entire Research Project}

On the following, I present my biggest challenges before and during the project, as well as some surprises I had as a person and researcher. I also report my learning about SSCs, and I talk about what went well in the project and what was not so good. Finally, I conclude by commenting on how my assumptions and personal values may have 
influenced the nature of my research, especially in the discussions of the Action Learning Set.

\subsubsection{My Biggest Challenges}

Since I started the DBA, I knew that the subject of my research would be the SSCs in the public sector. After all, they have been my object of study since my academic masters in 2010, and I wanted to deepen my knowledge and research in this area. However, it is when we put our hands at work that the real difficulties arise. From the existence of few studies carried out around the research sought, to the choice of approaches and methods, passing through the research locus. These three circumstances frightened me for some weeks and represented, perhaps, my great challenges, as I share below.

Only a few studies on Shared Services Centre - The existence of only a few studies on SSC was a challenge (even less in the public sector). Mainly, by the absence of references to having as a starting point on which to build. However, the challenge is increased when there are nearly no quantitative studies in the SSC universe, with conceptual models and measurement scales previously developed and empirically confirmed. Some may argue that this is also a great opportunity, which is true. However, it ends up taking a longer time than one might initially think to develop a conceptual model from scratch. This challenge led me to have at least three extra months on schedule about what I had planned.

Choice of approaches and methods - During the planning of this thesis, I was a little surprised by the suggestion of my first supervisor to adopt an AR approach, not only qualitative but also quantitative. I was preparing myself for over two years for an 
eminently qualitative approach and, I must confess, surprise caught me. I was reluctant initially to follow her suggestion, mainly because the quantitative approach was out of my comfort zone. Then, I realised it would be an opportunity for learning and development, for example, of my statistical skills, and went ahead to include the quantitative approach. This was indeed a wise decision, and I feel today a more complete researcher, able to conduct research using qualitative and quantitative approaches and methods.

Research locus - One concern that accompanied me during the months that preceded the research planning was, as an independent management consultant, not having an organisation where I could act as an inside researcher. This upset me a lot until I discussed it with my supervisor and proposed to involve people from other organisations, through an Action Learning Set, that could help in the development of knowledge, validate its applicability and turn that knowledge into action. In the end, this path proved to be very useful, both in generating new knowledge and in addressing actionable knowledge. It was also a way to bridge the theory-practice divide.

\subsubsection{What Surprised Me}

Two things surprised me in the cycle from the preparation to the completion of the research. The first was the ethical and technical rigour demanded by the University of Liverpool for conducting management research involving people. The legitimate concern with adequate information and obtain authorisation of the participants, a guarantee of anonymity, mandatory password and other care with information stored in a computer, etc. Similarly, the Ethics Committee authorizing the protocol and accompanying the development of the research, caught my attention. It was a valuable learning experience that I will take for the entire career of a researcher. The second 
surprise was the degree of seniority of the participants in the Action Learning Set. I decided to seek out the best, but I was unsure whether I would succeed. For example, I invited an SSC expert, renowned and pioneered in SSC deployment in the UK public sector, and he accepted the invitation. I also invited other senior executives and teachers and obtained seven ALS members who had all the seniority, knowledge, and experience requirements I defined as critical factors for the quality of knowledge generated in this research. This level of expertise was providential and wonderful.

\subsubsection{What Went Well and What Went Less Well}

As an example of what went well, I mention the back-up of high-level professionals at the University of Liverpool as my first and second supervisors and examiners. Also, the support offered, that is, all software required for research, availability of knowledge bases, access to practically all the knowledge on management existent, etc. As an example of what went less well, I mention my delay in recognising that it would need to expand the project scope of the public-sector SSC to public and private sector SSCs. The expansion of scope only occurred when I realised that just with public sector SSCs, I would not have the minimum number of respondents to ensure a quality sample for the quantitative part of the survey. I lost months in this indecision until I expanded the scope and it worked and got, even more, respondents than I needed (see Section 3.6 Method of Survey). I should have set a deadline for a decision-making if I did not have all the required sample.

\subsubsection{Personal Assumptions and Values Influencing the Research}

All research is subject to direct influences of the researcher's way of being and thinking, which to some extent, is natural. On the other hand, we must avoid the occurrence of the so-called 'researcher bias,' which occurs when the researcher obtains 
from the research the results that he or she would like to obtain (Burke, 1997). To avoid such a bias, I followed the whole protocol established for the quantitative part of the research and detailed the entire qualitative research procedure, to ensure external validation, as recommended by Creswell (2013). I also applied critical self-reflection (Burke, 1997), Checking systematically whether any potential bias or predisposition of mine occurred during the process or conclusion of the research. For example, in analysing the qualitative part of the research, I attempted to identify technically, through content analysis, what the respondents were saying and not what I thought I understood at first what they meant. These ways of minimizing the chances of researcher bias to occur were also a great learning experience, which is worth noting here.

\subsection{Reflections on Each Step of the Research Project}

As the Figure 3.9 below shows, the first cycle (Thesis research) has most of the activities conducted by the researcher with some independence during the production of the first draft of his thesis. The exception is the activity 2. Action/fieldwork, which triggers the second cycle (Survey and Action Learning Set - ALS), when it occurs, the interaction of the investigator with the other stakeholders interested in the research problem solution. In this research, the stakeholders are the respondents of the survey and the Action Learning Set (ALS) members. Once completed, the first and second cycles, the third cycle (Writing thesis), which also takes place with a certain independence of the researcher, is activated, and in this thesis, resulting in eight measures for improvement and adjustment of SSCs model to the context of the public sector. In addition, the third cycle also resulted in an action plan for the implementation of the 
measures, with recommendations to managers of civil SSC. Below, I discuss my learning and development, in each of these three cycles, following the logic of Figure 3.9.

Figure 3.9 Self-reflection and learning roadmap based on (Coghlan \& Brannick, 2014)

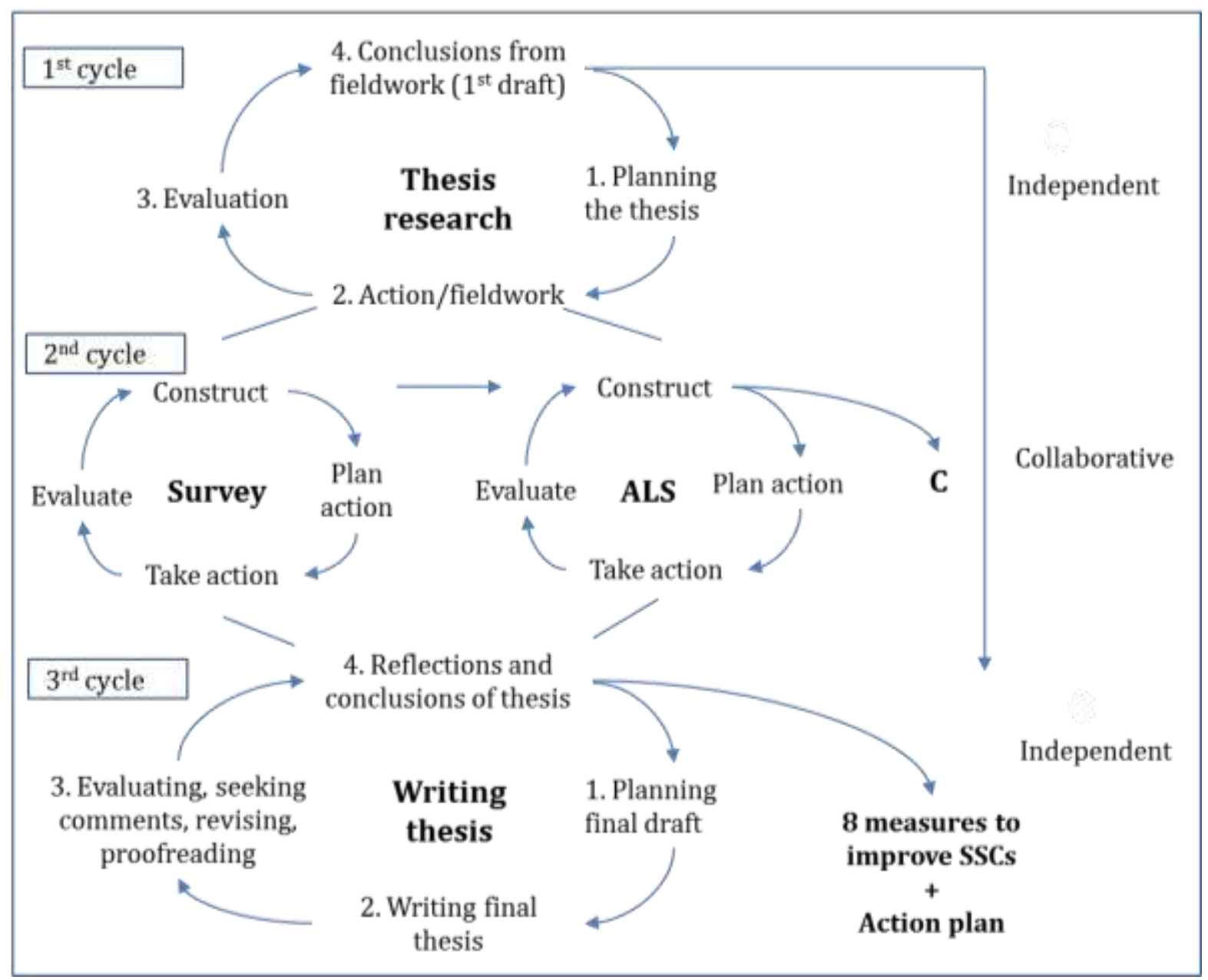

\subsubsection{First cycle - Thesis Research}

During the thesis planning, I identified that, although I was a management consultant who had led or participated in numerous SSC projects in the industry, I would not have all the necessary skills to analyse the results of research focused on the public sector, and extract the best findings. As argued by Mohrman et al. (2001), the usefulness of knowledge is related to the quality of interpretation of research results. For this reason, I began to idealise the use of skills of experienced researchers in the SSC theme 
in the public sector, as well as SSC managers, which eventually led to an Action Learning Set (ALS), formed by professionals and academics with outstanding expertise (as discussed in the previous sections). This type of 'joint interpretive forum' contributes to collectively reflect and interpret the information (Mohrman et al., 2001), and sense making. Work with the experts in ALS made it possible to ensure the appropriate level of quality of the interpretive research results, and the applicability of the knowledge developed for the target context (SSCs public sector). I was afraid of not getting people with the right profile to compose the ALS. However, as commented in item 5.2.2, the seniority and the effective contribution of the ALS were excellent.

\subsubsection{Second Cycle - Survey, and Action Learning Set - ALS}

Before applying the survey through a questionnaire, it was necessary to develop a conceptual model to express the main variables related to the performance of a SSC. This was one of my main issues, finding references of other empirical studies that were appropriate, as there are just a few empirical studies on SSCs. The apprenticeship was proportional to the size of the challenge, and I learnt how to compose a conceptual model from other analogous inquiries (even related to other objects of management), already validated empirically. Once prepared the conceptual model, with its constructs and measurement scales, the questionnaire was drawn up, and it was now necessary to find the right sample of SSCs to participate in the survey. The sampling criterion adopted was intentionality (Gil, 2002). I had a database of United Kingdom's public SSCs, with hundreds of contacts from its managers, and thought it would be relatively straightforward to have enough respondents. New learning. It was not like that, and I had low adherence to my invitation to participate in the survey. It was then necessary to expand the sample geographically to countries such as Brazil, Canada, the UK, and the 
US and include SSCs of the private sector. This ended up being very good because it brought plurality to the study data and enabled a direct comparison between SSCs of the public and private sectors. During the solution of this problem, I found numerous specialised SSC groups (public and private) on LinkedIn and even resumed contact with SSCs professionals from my network. Besides that, the discussion rounds with ALS members were very rich in learning. For example, one of the ALS comments was that the public affairs should not be managed as a business because the logic and purpose of government are different from the logic and business end. I remembered that I had read something similar in the literature review and this caused me deep reflection. Given my background in the industry, I was happy to have someone with an expert profile in public service to remember this kind of important principle. Another learning came from a comment from an ALS member noting that in her country, there is practically no negative political interference in the management of SSCs. This reality is so far away in countries like my Brazil. I remembered that one of the few experiences with SSCs in government in Brazil, after a successful implementation regarding results and reduced costs, was 'frozen' so that a different political party assumed the State Government responsible for that SSC, only due to an ideological matter. Moreover, that another SSC project, in another State, didn't go ahead due to different political interests between the potential secretaries of State participants. Another learning I had, occurred when a participant in the ALS suggested that the conceptual model could be transformed into part of a diagnostic framework. I had at no time thought of this and perhaps would never think of it. These experiences further reinforced the importance of having several experienced pairs of eyes helping to look and reflect on our work. Maybe to know that working in an Action Learning Set is such a wealth of experience, is my primary learning. Another learning is that a Virtual Action Learning Set (Pedler, 2011), as was the case of 
this thesis, works perfectly. Since the experts could participate at a time most convenient to them, it was possible to obtain the contribution of participants with the highest skills.

\subsubsection{Third cycle - Writing thesis}

This cycle favours the application of the knowledge window proposed for Coghlan \& Brannick (2014), that helps reflect on 1) what we know we know, 2) what we think we know, 3) what we know that we don't know, and 4) what we don't know we don't know. For example, 1) about what I knew I knew. On entering the research for this thesis, I knew my knowledge about the SSCs was good, both for industry (where I implemented many SSCs, working at PricewaterhouseCoopers and IBM GBS), and public service (which was the subject of my master's thesis). Looking back, my perception is that this knowledge, overall, has evolved let's say from seven to eight on a scale of zero to ten. However, knowledge about the public sector (in the context of this research) has evolved significantly, say from five to seven. 2) As for what I thought I knew, but were only assumptions and not facts, four examples are worth mentioning: (a) The origin of the SSCs dates back to the 1960s and not 1990s (see Section 2.3), as generally referred to; (b) Action Research (AR) can in practice help cover the gap theory-practice divide (Lee \& Greenley, 2010); (c) I understood well the differences between the contexts of the SSCs in the public and private sectors, and (d) Having a supervisor with quantitative background and another with qualitative background I could learn to work better with these two approaches and how to combine them in an AR project. 3) Regarding what I knew that I did not know, I think two outstanding examples were the new knowledge on statistic, to deal with the validation of the conceptual model, and the use of Action Research. The knowledge that, probably, it will be useful for my whole life. Finally, as for 
what 4) I did not know that I did not know, I can quote my discovery that I can work very well with statistics applied to research. Initially, I thought I would have much more difficulty on that; perhaps a little fear of the unknown.

\subsection{Future Research}

This research concentrated its efforts on examining the factors that influence the performance of SSCs, and the differences between the SSCs of the private and public sectors. This analysis resulted in the identification of eight measures that can contribute to the correct implementation and management of a public service SSC. Other measures may be identified. The results and conclusions obtained by this research, although they have generated knowledge that can be put in place immediately, also pave the way for new research on the public sector SSCs. As mentioned in Chapters Introduction and Literature Review, very little research has been conducted on the SSCs of the public service, although they represent a source of billions of dollars in cost reduction for governments that adopt them as government policy. As the trend continues to be the expansion of public SSCs, the time is opportune for more research to help public managers in identifying opportunities of SSC adoption, in projects ranging from the conception to the implementation, and on management and improvement of the services offered.

It has been found, for example, that some so-called SSCs in the public sector, does not present the necessary characteristics so that they can be categorized as SSCs. For instance, they do not collect for the given services (chargeback), they are not orientated to the clients, are not organised like unities of services, with relative independence, among others. In short, they are not SSCs, but departments that centralised activities that were carried out by government agencies, decentralized. Research in this area, to 
understand the motivations and offer alternative ways, possibly can help such departments to become effective SSCs. Another opportunity from the evidence of this study is that, although the New Public Management has encouraged the adoption of SSCs by governments-so that services to citizens could achieve quality levels like those of the private sector-was found that, although there are SSCs with high-quality service in some governments, this does not seem to be a predominant reality. This provides an opportunity for understanding the extent to which the NPM has failed, or not, to achieve this objective and why this occurred. 
REFERENCES 


\section{References}

Accenture (2013) High performance outcomes: Government shared services. [Online]. Available from https://www.accenture.com/t20160513T023713_w_/usen/_acnmedia/PDF-18/Accenture-High-Perfromance-Outcomes-POV.pdf [Accessed: 20 July 2016].

Agresti, A. \& Agresti, B.F. (2009) Statistical Methods for the Social Sciences. 4th Ed. London: Prentice Hall.

Ahire, S.L., Golhar, D.Y. \& Waller, M.A. (1996) Development and validation of TQM implementation constructs. Decision Sciences. 27 (1). pp. 23-53.

Allison, G.T. (1992) 'Public and private management: Are they fundamentally alike in all unimportant respects?', Classics of Public Administration. Belmont: C. A. Wordsworth, pp. 457-474.

Alt, R. \& Smits, M. (2007) 'Networkability of organizations and business networks', European Conference on Information Systems. St Gallen, Switzerland, 2007, pp. 119130.

Anderson, D. \& Anderson, L.A. (2010) Beyond change management: How to achieve breakthrough results through conscious change leadership. John Wiley \& Sons.

Armstrong, J.S. \& Overton, T.S. (1977) Estimating nonresponse bias in mail surveys. Journal of marketing research. pp. 396-402.

Armstrong, R., Waters, E., Dobbins, M., Anderson, L., Moore, L., Petticrew, M., Clark, R., Pettman, T.L., Burns, C., Moodie, M., Conning, R. \& Swinburn, B. (2013) Knowledge translation strategies to improve the use of evidence in public health decision making in local government: intervention design and implementation plan. Implementation Science. 8 (1). pp. 121-131.

Ashkenas, R. (1995) The Boundaryless Organization: Breaking the Chains of Organizational Structure. The Jossey-Bass Management Series. ERIC.

Augusto, M. \& Coelho, F. (2009) Market orientation and new-to-the-world products: Exploring the moderating effects of innovativeness, competitive strength, and environmental forces. Industrial Marketing Management. 38 (1). pp. 94-108. [Online]. DOI: $10.1016 /$ j.indmarman.2007.09.007.

Bagozzi, R.P. \& Yi, Y. (1988) On the evaluation of structural equation models. Journal of the academy of marketing science. 16 (1). pp. 74-94.

Baker, W.E. \& Sinkula, J.M. (1999) The Synergistic effect of Market Orientation and Learning Orientation on Organizational Performance. Journal of Academy of Marketing Science. 27 (4). pp. 411-427.

Baldwin, J.N. (1987) Public versus Private: Not That Different, Not That consequential. Public Personnel Management. 16 (2). pp. 181-193.

Bangemann, T.0. (2005) Shared services in finance and accounting. Gower Publishing, Ltd. 
Bardin, L. (1977) Análise de conteúdo. Paris: Presses Universitaires de France.

Barney, J. (1991) Firm resources and sustained competitive advantage. Journal of management. 17 (1). pp. 99-120.

Barton, J., Stephens, J. \& Haslett, T. (2009) Action research: its foundations in open systems thinking and relationship to the scientific method. Systemic Practice and Action Research. 22 (6). pp. 475-488.

Bearden, W.O. \& Netemeyer, R.G. (1999) Handbook of marketing scales: Multi-item measures for marketing and consumer behavior research. Sage.

Becker, J.J., Krause, A. \& Becker, J.J. (2009) Shared Services Strategies and Their Determinants : A Multiple Case Study Analysis in the Public Sector. Americas Conference on Information Systems (AMCIS). pp. 1-11.

Bergeron, B. (2002) Essentials of shared services. John Wiley \& Sons.

Borman, M. (2006) The Design and Success of Shared Services Centres. Business. (6). pp. 2209-2220.

Borman, M. \& Janssen, M. (2012) Reconciling two approaches to critical success factors: The case of shared services in the public sector. International Journal of Information Management. 33 (2). pp. 390-400.

Bou-Llusar, J.C., Escrig-Tena, A.B., Roca-Puig, V. \& Beltrán-Martín, I. (2009) An empirical assessment of the EFQM Excellence Model: Evaluation as a TQM framework relative to the MBNQA Model. Journal of Operations Management. 27 (1). pp. 1-22.

Boyne, G.A. (2002) Public and private management: what's the difference? Journal of Management Studies. (January). pp. 97-122.

Boyne, G.A. (2003) What is public service improvement? Public Administration. 81 (2). pp. 211-227.

Brand, A. (2006) The Politics of Shared Services. London.

Bridelli, S., Werneck, S. \& Martins, F. (n.d.) Centros de Serviços Compartilhados: Uma Solução Definitiva para os Processos Administrativos? (Shared Services Centers: A Definitive Solution for the Administrative Processes?). São Paulo [Online]. Available from http://www.bain.com/bainweb/PDFs/cms/Public/Centros_de_servicos_compartilhad os_Portuguese.pdf [Accessed: 13 June 2016].

Brocke, J. vom \& Rosemann, M. (2014) Handbook on Business Process Management 1: introduction, methods, and information systems.

Brown, S.L. \& Eisenhardt, K.M. (1997) The Art of Continuous Change: Linking Complexity Theory and Time-paced Evolution in Relentlessly Shifting Organizations. Administrative Science Quarterly. 42 (1). pp. 1-34.

Brown, T.A. (2015) Confirmatory factor analysis for applied research. Boston: Guilford Publications. 
Brunsdon, D. \& Dalziell, E. (2005) Making organisations resilient: understanding the reality of the challenge.

Bühler, A., Bühler, A., Wallenburg, C.M., Wallenburg, C.M., Wieland, A. \& Wieland, A. (2016) Accounting for external turbulence of logistics organizations via performance measurement systems. Supply Chain Management: An International Journal. 21 (6). pp. 694-708.

Burke, J.R. (1997) Examining the validity structure of qualitative research. Education. 118 (2). pp. 282-293.

Bywater (2001) Realizing the potential of shared services [Online]. Available from http://www.bywaterpartners.com/briefpss.htm [Accessed: 13 June 2016].

Camarero, C. (2007) Relationship orientation or service quality?: What is the trigger of performance in financial and insurance services? International Journal of Bank Marketing. 25 (6). pp. 406-426.

Cameron, K.S. \& Quinn, R.E. (2005) Diagnosing and changing organizational culture: Based on the competing values framework. John Wiley \& Sons.

Cano, C.R., Carrillat, F. a. \& Jaramillo, F. (2004) A meta-analysis of the relationship between market orientation and business performance: evidence from five continents. International Journal of Research in Marketing. 21 (2). pp. 179-200. [Online]. Available from http://linkinghub.elsevier.com/retrieve/pii/S0167811604000151.

Caruana, A., Pitt, L. \& Berthon, P. (1999) Excellence-Market Orientation Link: Some Consequences for Service Firms. Journal of Business Research. 15 (1). pp. 5-15.

Celuch, K.G., Kasouf, C.J. \& Peruvemba, V. (2002) The effects of perceived market and learning orientation on assessed organizational capabilities. Industrial Marketing Management. 31 (6). pp. 545-554. [Online]. DOI: 10.1016/S0019-8501(02)00187-6.

Cervera, A., Mollá, A. \& Sánchez, M. (2001) Antecedents and consequences of market orientation in public organisations. European Journal of Marketing. 35 (11). pp. 12591286.

Chang, T.-Z. \& Chen, S.-J. (1998) Market orientation, service quality and business profitability: a conceptual model and empirical evidence. Journal of Services Marketing. 12 (4). pp. 246-264.

Child, D. (2006) The essentials of factor analysis. 3rd Ed. London: Continuum.

Choi, M. \& Ruona, W.E.A. (2010) Individual readiness for organizational change and its implications for human resource and organization development. Human Resource Development Review. pp. 46-73.

Cinite, I., Duxbury, L.E. \& Higgins, C. (2009) Measurement of perceived organizational readiness for change in the public sector. British Journal of Management. 20 (2). pp. 265-277.

Clark, G., Johnston, R. \& Shulver, M. (2000) Exploiting the service concept for service design and development. New Service Design.Sage, Thousand Oaks, CA. pp. 71-91. 
Coghlan, D. \& Brannick, T. (2014) Doing action research in your own organization. Thousand Oaks: Sage Publications.

Couper, M.P. (2000) Web surveys - A review of issues and approaches. Public opinion quarterly. 64 (4). pp. 464-494.

Couper, M.P., Traugott, M.W. \& Lamias, M.J. (2001) Web survey design and administration. Public opinion quarterly. 65 (2). pp. 230-253.

Creswell, J.W. (2007) Qualitative inquiry \& research design: choosing among five approaches. Thousand Oaks: Sage Publications.

Creswell, J.W. (2013) Research design: Qualitative, quantitative, and mixed methods approaches. 3d Ed. Thousand Oaks: Sage publications.

Creswell, J.W. (1994) Research Design: qualitative and quantitative approaches. Thousand Oaks: Sage Publications.

Creswell, J.W. \& Clark, V.L.P. (2007) Designing and conducting mixed methods research. Thousand Oaks, Calif: Sage Publications.

Culyer, A.J. (2014) Encyclopedia of health economics. London: Elsevier.

Dale, B.G., Zairi, M., der Wiele Van \& Williams, A.R.T. (2000) Quality is dead in Europe long live excellence - true or false? Measuring Business Excellence. 4 (3). pp. 4-10.

Dancey, C.P. \& Reidy, J. (2007) Statistics without maths for psychology. London: Pearson Education.

Day, G.S. (1994) The Capabilities of Market-Drive Organizations. Journal of Marketing. 58 (4). pp. 37-52.

DeBlassie, R.R. \& Ludeman, M. (1973) Guidance Programs and Practices in Rural and Small Schools. National Educational Laboratory Publishers, Inc.

Deloitte (2007) Centros de serviços compartilhados: Tendências em um modelo de gestão cada vez mais comum nas organizações [Shared Service centres: Trends in a management model increasingly common in organisations.]. São Paulo.

Denhardt, J.V. \& Denhardt, R.B. (2007) The new public service: serving, not steering. Armonk, N.Y. : M.E. Sharpe, c2007; Expanded ed.

Denhardt, R.B. \& Denhardt, J.V. (2000) The New Public Service: Serving Rather than Steering. Public Administration Review. 60 (6). pp. 549-559.

Deshpande, R. \& Farley, J. (1998) Measuring Market Orientation: Generalization and Synthesis. Journal of Market-Focused Management. 2 (3). pp. 213-232. [Online]. Available from http://search.ebscohost.com.ezproxy.liv.ac.uk/login.aspx?direct=true \&db=edsbl\&AN= RN039228555\&site=eds-live\&scope=site.

Deshpande, R. \& Farley, J.U. (1996) Understanding market orientation: A prospectively designed meta-analysis of three market orientation scales. Marketing Science Institute 
Cambridge Massachusetts.

Deshpandé, R. \& Farley, J.U. (2004) Organizational culture, market orientation, innovativeness, and firm performance: An international research odyssey.

International Journal of Research in Marketing. 21 (1). pp. 3-22.

Deshpandé, R., Farley, J.U., Jr., F.E.W., Deshpande, R., Farley, J.U. \& Webster, F.E. (1993) Corporate Culture, Customer Orientation, and Innovativeness in Japanese Firms: A Quadrad Analysis. Journal of Marketing. 57 (January). pp. 23-27. [Online]. DOI: $10.2307 / 1252055$.

Dessein, W. \& Santos, T. (2006) Adaptive organizations. Journal of Political Economy. 114 (5). pp. 956-995.

Dillman, D.A., Smyth, J.D. \& Christian, L.M. (2014) Internet, phone, mail, and mixed-mode surveys: the tailored design method. John Wiley \& Sons.

Dunleavy, P. \& Hood, C. (1994) From old public administration to new public management. Public Money \& Management. 14 (3). pp. 9-16.

Easterby-Smith, M., Thorpe, R. \& Jackson, P.R. (2008) Management research. 3d. Sage (ed.) London: SAGE, 2008.

EFQM (2015) What is Excellence? 2015 (7/11/2015). [Online]. Available from http://www.efqm.org/efqm-model/what-is-excellence.

Eisenhardt, K.M. \& Martin, J.A. (2000) Dynamic capabilities: what are they? Strategic Management Journal. 21 (10-11). pp. 1105-1121.

Estabrooks, C., Thompson, D.S., Lovely, J.J.E. \& Hofmeyer, A. (2006) A guide to knowledge translation theory. The Journal of continuing education in the health professions. 26 (1). pp. 25-36.

Evans, J.R. (2013) Quality \& performance excellence. Cengage Learning.

Evanschitzky, H. (2007) Market orientation of service networks: direct and indirect effects on sustained competitive advantage. Journal of Estrategic Management. 368 (September). pp. 349-369.

Farquhar, C.R., Fultz, J.M. \& Graham, A. (2006) Implementing Shared Services in the Public Sector: The Pillars of Success. Conference Board of Canada.

Fersht, P. \& Aird, C. (2011) The Evolution of Global Business Services: Enhancing the Benefits of Shared Services and Outsourcing. HfS Research. (July). pp. 1-19.

Field, A. (2009) Discovering statistics using SPSS. Thousand Oaks: Sage Publications.

Flynn, B.B., Schroeder, R.G. \& Sakakibara, S. (1994) A framework for quality management research and an associated measurement instrument. Journal of Operations management. 11 (4). pp. 339-366.

Flynn, N. (2007) Public sector management. Sage.

Fok-Yew, O. \& Ahmad, H. (2014) Management of Change and Operational Excellence in 
the Electrical and Electronics Industry. 3 (2).

Forza, C. (2002) Survey research in operations management: a process-based perspective. International Journal of Operations \& Production Management. 22 (2). pp. 152-194.

Frei, F.X. (2006) Breaking the trade-off between efficiency and service. Harvard business review. 84 (11). pp. 93-101.

Frei, F.X. (2008) The Four Things a Service Company Must Get Right. Harvard Business Review. 86 (4). pp. 70-80.

Gainer, B. \& Padanyi, P. (2005) The relationship between market-oriented activities and market-oriented culture: Implications for the development of market orientation in nonprofit service organizations. Journal of Business Research. 58 (6). pp. 854-862.

Gebhardt, G.F., Carpenter, G.S. \& Sherry Jr, J.F. (2006) Creating a Market Orientation: A Longitudinal, Multifirm, Grounded Analysis of Cultural Transformation. Journal of Marketing. 70 (4). pp. 37-55.

George, D. \& Mallery, P. (2003) SPSS for Windows step by step: a simple guide and reference. 11.0 update. 4th Ed. Boston: Allyn \& Bacon.

Gershon, P. (2004) Releasing resources to the front line: Independent Review of Public Sector Efficiency. HM Stationery Office.

Gil, A.C. (2002) Como Elaborar Projetos de Pesquisa [How to develop research projects]. 4a Ed. Sao Paulo: Editora Atlas.

Goff, P. Le (2005) Shared Services: Lower Costs, Improved Services and a Change in Culture. Parliamentary Information and Research Service [Online]. Available from http://books.google.com.br/books?id=KMBWnQEACAAJ.

Goldstein, S.M., Johnston, R., Duffy, J. \& Rao, J. (2002) The service concept: the missing link in service design research? Journal of Operations Management. 20 (2). pp. 121134.

Gonzalez, R., Llopis, J. \& Gasco, J. (2013) Innovation in public services: The case of Spanish local government. Journal of Business Research. 66 (10). pp. 2024-2033.

Gould, K., Gould, K., Partner, A., Partner, A., Magdieli, A., Magdieli, A., Consultant, M. \& Consultant, M. (2007) Optimizing government effectiveness through shared services: Perspectives from IBM Corporation. IBM Global Business Services. (April).

Gouthier, M., Giese, A. \& Bartl, C. (2012) Service excellence models: a critical discussion and comparison. Managing Service Quality: An International Journal. 22 (5). pp. 447464.

Gouthier, M., Giese, A. \& Bartl, C. (1994) Service excellence models: a critical discussion and comparison. Managing Service Quality. 22 (5). pp. 447-464.

Greenley, G.E. (1995) Market Orientation and Company Performance : Empirical Evidence From UK Companies. British Journal of Management. 6 (1). pp. 1-13. 
Greenwood, D.J. (2007) Pragmatic action research. International Journal of Action Research. 3 (1+2). pp. 131-148.

Greenwood, D.J. \& Levin, M. (2006) Introduction to action research: Social research for social change. Thousand Oaks: Sage Publications.

Grewal, R. \& Tansuhaj, P. (2001) Building organizational capabilities for managing economic crisis: The role of market orientation and strategic flexibility. Journal of Marketing. 65 (2). pp. 67-80.

Grönroos, C. (2011) Value co-creation in service logic: A critical analysis. Marketing theory. 11 (3). pp. 279-301.

Haffar, M., Al-Karaghouli, W. \& Ghoneim, A. (2013) The mediating effect of individual readiness for change in the relationship between organisational culture and TQM implementation. Total Quality Management and Business Excellence. 24 (5-6). pp. 693706.

Hair, J.F. (2006) Multivariate Data Analysis. Pearson international edition Pearson Prentice Hall [Online]. Available from https://books.google.com.br/books?id=WESxQgAACAAJ.

Hair, J.F. (1998) Multivariate data analysis. P. Hall (ed.) Upper Saddle River, N.J : Prentice Hall, c1998. [Online]. Available from https://liverpool.idm.oclc.org/login?url=http://search.ebscohost.com/login.aspx?dire ct=true \&db=cat00003a\&AN=lvp.b1774447\&site=eds-live \&scope=site.

Halasz, G. (2016) 'School-university partnership for effective teacher learning', KIP Regional Methodological Centre. 2016.

Hammer, M. (2007) The Process Audit. Harvard business review. 85 (4). pp. 111-123. [Online]. Available from http://search.ebscohost.com.ezproxy.liv.ac.uk/login.aspx?direct=true \&db=bth\&AN=24 $267684 \&$ site $=$ eds-live $\&$ scope $=$ site.

Han, J.K., Kim, N. \& Srivastava, R.K. (1998) Market orientation and organizational performance: is innovation a missing link? Journal of Marketing. 62 (4). pp. 30-45.

Harris, L.C. \& Ogbonna, E. (2001) Leadership style and market orientation: an empirical study. European journal of marketing. 35 (5/6). pp. 744-764.

Hayden, V. (1993) How to increase market orientation. Journal of management in medicine. 7 (1). pp. 29-46.

Herr, K. \& Anderson, G.L. (2014) The action research dissertation: A guide for students and faculty. Sage publications.

Hietschold, N., Reinhardt, R. \& Gurtner, S. (2014) Measuring critical success factors of TQM implementation successfully - a systematic literature review. International Journal of Production Research. 52 (July). pp. 1-19.

Hofstede, G. (1984) Culture's consequences: International differences in work-related values. sage. 
Homburg, C. \& Pflesser, C. (2000) A Multiple-Layer Model of Market-Oriented Organizational Culture: Measurement Issues and Performance Outcomes. Journal of Marketing Research. 37 (4). pp. 449-462.

Hood, C. (1995a) Contemporary public management: a new global paradigm? Public policy and administration. 10 (2). pp. 104-117.

Hood, C. (1995b) The 'New Public Management' in the 1980s: variations on a theme. Accounting, organizations and society. 20 (2). pp. 93-109.

Hood, C. \& Peters, G. (2013) The Middle Aging of New Public Management: Into the Age of Paradox? Journal of Public Administration Research and Theory. 14 (3). pp. 267-282.

Hughes, O.E. (2012) Public management and administration. Palgrave Macmillan.

Hult, G.T.M. (2011) Market-focused sustainability: Market orientation plus! Journal of the Academy of Marketing Science. 39 (1). pp. 1-6.

Hult, G.T.M. \& Ketchen, D.J. (2001) Does market orientation matter?: A test of the relationship between positional advantage and performance. Strategic Management Journal. 22 (9). pp. 899-906.

Hult, G.T.M., Ketchen Jr, D.J. \& Slater, S.F. (2005) Market Orientation and Performance: an integration of disparate approaches. Strategic Management Journal. 26 (12). pp. 1173-1181.

Irani, Z., Beskese, A. \& Love, P.E.D. (2004) Total quality management and corporate culture: constructs of organisational excellence. Technovation. 24. pp. 643-650. [Online]. Available from http://10.0.3.248/S0166-4972(02)00128-1.

Jansen, K.J. (2000) The Emerging Dynamics of Change: Resistance, Readiness, and Momentum. Human Resource Planning. 23 (2). pp. 53-55.

Janssen, M. \& Joha, A. (2006) Motives for establishing shared service centers in public administrations. International Journal of Information Management. 26 (2). pp. 102-115.

Janssen, M. \& Joha, A. (2010) Public-private partnerships, outsourcing or shared service centres? Motives and intents for selecting sourcing configurations. Transforming Government: People, process and policy. 4 (3). pp. 232-248.

Janssen, M. \& Joha, A. (2008) The Strategic Determinants of Shared Services Public Information Technology Handbook. Handbook of Research on Public Information Technology. II.

Janssen, M. \& Joha, A. (2007) Understanding IT governance for the operation of shared services in public service networks. International Journal of Networking and Virtual Organisations. 4 (1). pp. 20-34.

Janssen, M., Joha, A. \& Weerakkody, V. (2007) Exploring relationships of shared service arrangements in local government. Transforming Government. 1 (3). pp. 271-284.

Jaworski, B.J. \& Kohli, A.K. (1993) Market orientation: antecedents and consequences. The Journal of marketing. 57 (July). pp. 53-70. 
Johnson, R.B. \& Onwuegbuzie, A.J. (2004) Mixed methods research: A research paradigm whose time has come. Educational researcher. 33 (7). pp. 14-26.

Johnston, R. (2004) Towards a better understanding of service excellence. Managing Service Quality. 14 (2/3). pp. 129-133.

Johnston, R. \& Clark, G. (2001) Service Operations Management. London: Prentice-Hall. Jones, R.A., Jimmieson, N.L. \& Griffiths, A. (2005) The Impact of Organizational Culture and Reshaping Capabilities on Change Implementation Success: The Mediating Role of Readiness for Change. Journal of Management Studies. 42 (2). pp. 22-2380.

Jonker, J. \& Pennink, B.J.W. (2008) The essence of research methodology: a concise guide for master and PhD students in management science. New York: Berlin; Springer.

Kaiser, H.F. (1974) An index of factorial simplicity. Psychometrika. 39 (1). pp. 31-36.

Kalyan Acharjya (2016) Research Methodology and Research Design [Online]. Available from http://pt.slideshare.net/kalyanacharjya/research-methodology-kalyan-acharjya [Accessed: 21 July 2016].

Kanji, G.K. (1998) Measurement of business excellence. Total Quality Management. 9 (7). pp. 633-643.

Kanji, G.K. \& Sá, P.M. (2001) Measuring leadership excellence. Total Quality Management. 12 (6). pp. 701-718.

Kapucu, N. (2006) 'New Public Management: Theory, Ideology, and Practice', Ali Farazmand and Jack Pinkowski (ed.). Handbook of Globalization, Governance, and Public Administration. New York: Taylor \& Francis Group, pp. 889-902.

Keuper, F. (Hrsg. . \& Lueg, K.-E. (Hrsg. . (2013) Finance bundling and finance transformation : shared services next level. Wiesbaden [Online]. Available from http://search.ebscohost.com.ezproxy.liv.ac.uk/login.aspx?direct=true\&db=edszbw\&A $\mathrm{N}=\mathrm{EDSZBW726932825 \& \text {site=eds-live\&scope=site. }}$

Kincaid, J. (1997) Moore, Mark H. Creating Public Value: Strategic Management in Government. Journal of Politics. 59 (1). pp. 257-258.

Kirca, A.H., Jayachandran, S. \& Bearden, W.O. (2005) Market Orientation: A MetaAnalytic Review and Assessment of Its Antecedents and Impact on Performance. Journal of Marketing. 69 (2). pp. 24-41.

Klefsjö, B., Bergquist, B. \& Garvare, R. (2008) Quality management and business excellence, customers and stakeholders: Do we agree on what we are talking about, and does it matter? TQM Journal. 20 (2). pp. 120-129.

Klijn, E.-H.-H. (2008) Complexity Theory and Public Administration: What's New? Public Management Review. 10 (3). pp. 299-317.

Kohli, A.K. \& Jaworski, B.J. (1990) Market orientation: the construct, research propositions, and managerial implications. The Journal of Marketing. 54 (April). pp. 118. 
Kotter, J.P. (1995) Leading change: Why transformation efforts fail. Harvard business review. 73 (2). pp. 59-67.

Kotter, J.P. (2001) What Leaders Really Do. Harvard business review. 79 (11). pp. 85-96.

Krüger, G.P. (2012) Implementation of Shared Service Centers (SSC) in the State of Rio Grande do Sul (RS). p.pp. 1-41.

Kumar, V., Jones, E., Venkatesan, R. \& Leone, R.P. (2011) Is Market Orientation a Source of Sustainable Competitive Advantage or Simply the Cost of Competing? Journal of Marketing. 75 (1). pp. 16-30.

Lacity, M.C. \& Fox, J. (2008) Creating Global Shared Services: Lessons from Reuters. MIS Quarterly Executive. 7 (1). pp. 17-32.

Landry, R., Amara, N., Pablos-Mendes, A., Shademani, R., Gold, I., é. Landry, R., Amara, N., Pablos-Mendes, A., Shademani, R. \& Gold, I. (2006) The knowledge-value chain: a conceptual framework for knowledge translation in health. Bulletin of the World Health Organization. 84 (8). pp. 597-602.

Lear, R.W. (1963) No easy road to market orientation. Harvard business review : HBR. 41 (5). pp. 53-60.

Lee, N. \& Greenley, G. (2010) The theory-practice divide: thoughts from the Editors and Senior Advisory Board of EJM. European Journal of Marketing. 44 (1-2). pp. 5-20.

Liao, S.-H.H., Chang, W.-J.J., Wu, C.-C.C. \& Katrichis, J.M. (2011) A survey of market orientation research (1995-2008). Industrial Marketing Management. 40 (2). pp. 301310.

Liu, S. (2009) Organizational Culture and New Service Development Performance: Insights From Knowledge Intensive Business Service. International Journal of Innovation Management. 13 (3). pp. 371-392.

MacCallum, R.C., Widaman, K.F., Zhang, S. \& Hong, S. (1999) Sample size in factor analysis. Psychological methods. 4 (1). pp. 84-99.

Mahoney, J. \& Pandian, J. (1992) The Resource- Based View within the Conversation of Strategic Management. Strategic Management Journal. 13 (5). pp. 363-380.

Malhotra, M.K. \& Grover, V. (1998) An assessment of survey research in POM: from constructs to theory. Journal of Operations Management. 16 (4). pp. 407-425.

Malhotra, N.K. (2006) Questionnaire design and scale development. The handbook of marketing research: Uses, misuses, and future advances. pp. 83-94.

Marinova, D., Ye, J. \& Singh, J. (2008) Do Frontline Mechanisms Matter? Impact of Quality and Productivity Orientations on Unit Revenue, Efficiency, and Customer Satisfaction. Journal of Marketing. 72. pp. 28-45.

Marques, F. (2006) Guia prático de excelência em serviços [Practical guide for excellence in services]. Sao Paulo: NBL Editora. 
Matsuno, K., Mentzer, J.. T. \& Rentz, J.0. (2000) A refinement and validation of the MARKOR scale. Journal of the Academy of Marketing Science. 28 (4). pp. 527-539.

McNeill, P. \& Chapman, S. (2005) Research methods. Psychology Press.

Mechling, J. \& Schwarz, S.G. (2007) Shared service center. RAR-103, Kennedy School of Government, Harvard University, Student-Gary Schwarz.

Menguc, B. \& Auh, S. (2006) Creating a firm-level dynamic capability through capitalizing on market orientation and innovativeness. Journal of Academy of Marketing Science. 34 (1) p.pp. 63-73.

Menguc, B., Auh, S. \& Shih, E. (2007) Transformational leadership and market orientation: Implications for the implementation of competitive strategies and business unit performance. Journal of Business Research. 60 (4). pp. 314-321.

Menor, L.J. \& Roth, A. V. (2007) New service development competence in retail banking: Construct development and measurement validation. Journal of Operations Management. 25 (4). pp. 825-846. [Online]. DOI: 10.1016/j.jom.2006.07.004.

Merriam-Webster (2015a) Excellence / Definition of excellence by Merriam-Webster. 2015 (9/6/2015).

Merriam-Webster (2015b) Public Service / Definition of public service by MerriamWebster. 2015 (9/16/2015).

Meyer, J.P., Allen, N.J. \& Smith, C.A. (1993) Commitment to organizations and occupations: Extension and test of a three-component conceptualization. Journal of Applied Psychology. 78 (4). pp. 538-551. [Online]. DOI: 10.1037/0021-9010.78.4.538.

Mohammad Qawasmeh, F., Darqal, N. \& Farid Qawasmeh, I. (2013) The Role of Organization Culture in Achieving Organizational Excellence: Jadara University as a Case Study. International Journal of Economics and Management Sciences Culture. 2 (7). pp. 5-19.

Mohrman, S.A., Gibson, C.B. \& Mohrman, A.M. (2001) Doing research that is useful to practice a model and empirical exploration. Academy of Management journal. 44 (2). pp. 357-375.

Moran, J.W. \& Brightman, B.K. (2000) Leading organizational change. Journal of Workplace Learning. 12 (2). pp. 66-74.

Morgan, N. a., Vorhies, D.W. \& Mason, C.H. (2009) Market orientation, marketing capabilities and firm performance. Strategic Management Journal. 30 (8). pp. 909-920. [Online]. DOI: 10.1002/smj.764.

Mowday, R.T., Steers, R.M. \& Porter, L.W. (1979) The measurement of organizational commitment. Journal of Vocational Behavior. 14 (2). pp. 224-247. [Online]. DOI: 10.1016/0001-8791(79)90072-1 [Accessed: 2 May 2017].

Murray, J.Y., Gao, G.Y. \& Kotabe, M. (2011) Market orientation and performance of export ventures: The process through marketing capabilities and competitive advantages. Journal of the Academy of Marketing Science. 39 (2). pp. 252-269. [Online]. 
DOI: $10.1007 / s 11747-010-0195-4$.

NAO (2008) Shared services in the Department for Transport and its agencies. National Audit Office.

Narver, J.C. \& Slater, S.F. (1990) The effect of a market orientation on business profitability. The Journal of Marketing. (October). pp. 20-35.

Narver, J.C., Slater, S.F. \& MacLachlan, D.L. (2004) Responsive and proactive market orientation and new-product success. Journal of Product Innovation Management. 21 (5). pp. 334-347.

Narver, J.C., Slater, S.F. \& Tietje, B. (1998) Creating a Market Orientation. Journal of Market-Focused Management. 2 (3). pp. 241-255.

NCA (2015) Towards Responsible Government - The Report of the National Commission of Audit. 2015 (7/9/2015).

NLGN (2002) Advancing a new public service ethos. New Local Government Network London.

Noble, C.H. \& Mokwa, M.P. (1999) Implementing Marketing Strategies: Developing and Testing a Managerial Theory. Journal of Marketing. 63 (4). pp. 57-73.

Noble, C.H., Sinha, R.K. \& Kumar, A. (2002) Market Orientation and Alternative Strategic Orientations: A Longitudinal Assessment of Performance Implications. Journal of Marketing. 66 (4). pp. 25-39.

Nunnally, J.C. \& Bernstein, I.H. (1994) Psychometric theory. McGraw-Hill series in psychology New York; McGraw-Hill, 1994.

Oakland, J.S. (2001) Total organizational excellence: Achieving world-class performance. Routledge.

OECD (1995) Governance in transition: public management reforms in OECD countries. Organization for Economic Co-operation and Development.

OECD (2010) Value for Money in Government: Public Administration after New Public Management. Organization for Economic Co-operation and Development.

Oliveira, P. \& Roth, A. V (2012) Service orientation: the derivation of underlying constructs and measures. International Journal of Operations \& Production Management. 32 (1-2). pp. 156-190. [Online]. Available from http://search.ebscohost.com.ezproxy.liv.ac.uk/login.aspx?direct=true \&db=edswss\&AN $=000302510200007 \&$ site $=$ eds-live $\&$ scope $=$ site .

Osborne, D. (1993) Reinventing Government. Public Productivity \& Management Review VO - 16. (4). pp. 349-356. [Online]. Available from

https://liverpool.idm.oclc.org/login?url=http://search.ebscohost.com/login.aspx?dire $c t=$ true $\& \mathrm{db}=$ edsjsr \&AN=edsjsr.10.2307.3381012\&site=eds-live \&scope=site.

Osborne, S.P. (2010) Delivering Public Services: Time for a new theory? Public Management Review. 12 (1). pp. 1-10. 
Osborne, S.P. (2006) The new public governance? PUBLIC MANAGEMENT REVIEW. 8 (3). pp. 377-387.

Paagman, A., Tate, M., Furtmueller, E. \& de Bloom, J. (2015) An integrative literature review and empirical validation of motives for introducing shared services in government organizations. International Journal of Information Management. 35 (1). pp. $110-123$.

Pablo, A.L., Reay, T., Dewald, J.R. \& Casebeer, A.L. (2007) Identifying, enabling and managing dynamic capabilities in the public sector*. Journal of Management Studies. 44 (5). pp. 687-708.

Pallant, J. (2010) SPSS survival manual [electronic book]: a step by step guide to data analysis using SPSS. Online access with purchase: Dawsonera Maidenhead : McGrawHill, 2010. [Online]. Available from https://liverpool.idm.oclc.org/login?url=http://search.ebscohost.com/login.aspx?dire $\mathrm{ct}=$ true $\& \mathrm{db}=$ cat00003a $\& A N=$ lvp.b2499673\&site=eds-live \&scope=site.

Pedler, M. (2012) Action learning for managers. Gower Publishing, Ltd.

Pedler, M. (2011) Action learning in practice. Gower Publishing, Ltd.

Pedler, M.J. (2008) Action learning for managers. [electronic book]. Aldershot, England; Ashgate Pub., c2008. [Online]. Available from

https://liverpool.idm.oclc.org/login?url=http://search.ebscohost.com/login.aspx?dire $\mathrm{ct}=$ true $\& \mathrm{db}=$ cat00003a $\& A N=$ lvp.b2359349\&site=eds-live \&scope=site.

Peng, D., Schroeder, R. \& Shah, R. (2011) Linking routines to operations capabilities: A new perspective. Journal of operations management. [Online]. Available from http://www.sciencedirect.com/science/article/pii/S0272696307001441 [Accessed: 3 May 2017].

Persson, D. \& Göransson, P. (2012) The Diffusion of Shared Service Centers - Based on Rational Arguments or Trends? A Case Study of The Motives and Decision-Making Process at Four Organizations.

Pesch, U. (2008) The publicness of public administration. Administration and Society. 40 (2). pp. 170-193.

Peteraf, M.A. (1993) The cornerstones of competitive advantage: A resource-based view. Strategic Management Journal. 14 (3). pp. 179-191.

Peters, T.J., Waterman, R.H. \& Jones, I. (1982) In search of excellence: Lessons from America's best-run companies.

Pitt, L., Caruana, A. \& Berthon, P.R. (1996) Market orientation and business performance: some European evidence. International Marketing Review. 13 (1). pp. 518.

Podsakoff, P.M. \& Organ, D.W. (1986) Self-reports in organizational research: Problems and prospects. Journal of management. 12 (4). pp. 531-544.

Pollitt, C. (1990) Managerialism and the public services: The Anglo-American experience. 
Basil Blackwell.

Pollitt, C. \& Summa, H. (1997) Trajectories of Reform: Public Management Change in Four Countries. Public Money \& Management. 17 (1). pp. 7-18.

Porter, M.E. (1985) Competitive advantage: creating and sustaining superior performance. New York: Free Press.

Prabhu, V.B. \& Robson, A. (2000) Achieving service excellence - measuring the impact of leadership and senior management commitment. Managing Service Quality. 10 (5). pp. 307-317.

Price, R. \& Brodie, R.J. (2001) Transforming a Public Service Organization from inside Out to Outside in The Case of Auckland City, New Zealand. Journal of Service Research. 4 (1). pp. 50-59.

Pulendran, S., Speed, R., Ii, R.E.W. \& Widing, R.E. (2000) The antecedents and consequences of market orientation in Australia. Australian journal of management. 25 (2). pp. 119-143.

Quinn, B., Cooke, R. \& Kris, A. (2000) Shared services: mining for corporate gold. Financial Times, Prentice Hall.

Ramsey, C. (2014) Management learning: A scholarship of practice centred on attention? Management Learning. 45 (1). pp. 6-20.

Raue, J.S. \& Wieland, A. (2015) The interplay of different types of governance in horizontal cooperations: A view on logistics service providers. The International Journal of Logistics Management. 26 (2). pp. 401-423. [Online]. DOI: 10.1108/IJLM-082012-0083 [Accessed: 3 May 2017].

Reason, P. \& Bradbury, H. (2001) Handbook of action research: Participative inquiry and practice. Thousand Oaks: Sage Publications.

Rego, H., Da Hora, M., Torres, G., Monteiro, R. \& Arica, J. (2010) Confiabilidade em Questionários para Qualidade: Um Estudo com o Coeficiente Alfa de Cronbach [Reliability in Questionnaires for Quality: a study with the Cronbach's alpha Coefficient] [Online]. Available from

http://seer.ufrgs.br/index.php/ProdutoProducao/article/viewFile/9321/8252 [Accessed: 23 July 2016].

Richter, P.C. \& Brühl, R. (2016) Shared service center research: A review of the past, present, and future. European Management Journal. [Online]. Available from http://10.0.3.248/j.emj.2016.08.004.

Robbins, S.P. (2011) Organizational behavior. Upper Saddle River, NJ : Prentice Hall, 2011; 11th ed [Online]. Available from

http://search.ebscohost.com.ezproxy.liv.ac.uk/login.aspx?direct=true $\& d b=$ cat00003a \&AN=lvp.b1848848\&site=eds-live \&scope $=$ site.

Rogers, S., Lukens, S., Lin, S. \& Jon, E. (2007) Balancing Risk and Performance with an Integrated Finance Organization: The Global CFO Study 2008. IBM Corporation in 
cooperation with The Wharton School and Economist Intelligence Unit. October.

Rungtusanatham, M.J., Choi, T.Y., Hollingworth, D.G., Wu, Z. \& Forza, C. (2003) Survey research in operations management: historical analyses. Journal of Operations Management. 21 (4). pp. 475-488.

Said, H. \& Thuraya, F. (2016) Implementing performance management in Brunei: a case study of public sector reform.

Saunders, M.N.K. (2012) Research methods for business students. 6th Ed. Harlow: Pearson Education Limited.

Schein, E.H. (1999) The corporate culture survival guide.

Schulman, D.S., Harmer, M.J., Dunleavy, J.R. \& Lusk, J.S. (1999) Shared services: Adding value to the business units. Wiley New York, NY.

Schulz, V. \& Brenner, W. (2010) Characteristics of shared service centers. Transforming Government: People, Process and Policy. 4 (3). pp. 210-219. [Online]. Available from http://www.emeraldinsight.com.eaccess.ub.tum.de/journals.htm?issn=17506166\&volume $=1 \&$ issue $=4 \&$ articleid $=1631527 \&$ show $=$ html.

Schwarz, G. (2014) Public shared service centers: a theoretical and empirical analysis of US public sector organizations. Wiesbaden: Springer Gable. [Online]. Available from http://search.ebscohost.com.ezproxy.liv.ac.uk/login.aspx?direct=true $\& d b=c a t 00003 a$ \&AN=lvp.b3433598\&site=eds-live \&scope=site;

Schwarz, G. \& Chakraborty, A. (2012) Public Shared Service Centers: A Theoretical and Empirical Analysis of US Public Sector Organizations.

Searle, P. (2014) Exploding the Myths of Shared Services. [Online]. Available from http://uknowledgeshare.com/wp-content/uploads/Exploding-the-Myths-of-SharedServices.pdf [Accessed: 19 June 2016].

Seashore, S.E. \& Taber, T.D. (1975) Job satisfaction indicators and their correlates. The American Behavioral Scientist. 18 (3). pp. 333-368.

Seddon, J. (2008) Systems thinking in the public sector. [electronic book]: the failure of the reform regime... and a manifesto for a better way. Axminster, United Kingdom : Triarchy Press.

Sharma, S., Netemeyer, R.G. \& Mahajan, V. (1990) In search of excellence revisited: an empirical evaluation of Peters and Waterman's attributes of excellence. Enhancing knowledge development in marketing. 1. pp. 322-328.

Sijtsma, K. (2009) On the use, the misuse, and the very limited usefulness of Cronbach's alpha. Psychometrika. 74 (1). pp. 107-120. [Online]. Available from http://link.springer.com/article/10.1007/s11336-008-9101-0/fulltext.html.

Slater, S.F. \& Narver, J.C. (1994) Market orientation, customer value, and superior performance. Business Horizons. 37 (2). pp. 22-28.

Sole, F. \& Schiuma, G. (2010) Using performance measures in public organisations: 
challenges of Italian public administrations. Measuring Business Excellence. 14 (3). pp. 70-84.

Song, M. \& Parry, M.E. (2009) The desired level of market orientation and business unit performance. Journal of the Academy of Marketing Science. 37 (2). pp. 144-160.

Speklé, R.F. \& Verbeeten, F.H.M.M. (2014) The use of performance measurement systems in the public sector: Effects on performance. Management Accounting Research. 25 (2). pp. 131-146.

Statstutor (2016) Pearson's correlation [Online]. Available from http://www.statstutor.ac.uk/resources/uploaded/pearsons.pdf [Accessed: 23 July 2016].

Stratman, J.K. \& Roth, A. V (2002) Enterprise Resource Planning (ERP) Competence Constructs: Two-Stage Multi-Item Scale Development and Validation. Decision Sciences. 33 (4). pp. 601-628. [Online]. Available from http://search.ebscohost.com.ezproxy.liv.ac.uk/login.aspx?direct=true $\& d b=b t h \& A N=93$ $24984 \&$ site $=$ eds-live $\&$ scope $=$ site .

Straus, S.E., Tetroe, J. \& Graham, I. (2009) Defining knowledge translation. Journal of the Canadian Medical Association. 181 (3-4). pp. 165-168.

Stringer, E.T. (2013) Action research. London: Sage Publications.

Su, N. (2009) Shared Services Transformation: Conceptualization and Valuation from the Perspective of Real Options. 40 (3). pp. 381-403.

Susman, G.I. \& Evered, R.D. (1978) An assessment of the scientific merits of action research. Administrative science quarterly. pp. 582-603.

Syed-Ikhsan, S.O.S. \& Rowland, F. (2004) Knowledge management in a public organization: A study on the relationship between organizational elements and the performance of knowledge transfer. Journal of Knowledge Management. 8 (2). pp. 95111.

Tappenden, P. (2012) Conceptual modelling for health economic model development. HEDS Discussion Paper 12/05.

Teece, D. \& Pisano, G. (1994) The dynamic capabilities of firms: An introduction. Industrial and Corporate Change. 3 (3). pp. 537-556.

Teece, D.J. (2007) Explicating Dynamic Capabilities: The Nature and Microfoundations of (Sustainabile) Enterprise Performance. Strategic Management Journal. 298. pp. 1319-1350.

Theodosiou, M. \& Katsikea, E. (2013) The Export Information System: An Empirical Investigation of Its Antecedents and Performance Outcomes. Journal of International Marketing. 21 (3). pp. 72-94.

Thomaz, A.R. \& Gon, M.V. (2013) 'Centro de Serviços Compartilhados: a experiência do Estado de Minas Gerais [Shared Services Centre: the experience of the State of Minas Gerais]', XXVII Encontro da EnANPAD. Rio de Janeiro: EnANPAD, 2013, pp. 1- 
16.[Online]. Available from http://www.anpad.org.br/admin/pdf/2013_EnANPAD_APB974.pdf.

Todnem By, R. (2005) Organisational change management: A critical review. Journal of Change Management. 5 (4). pp. 369-380.

Tomaskova, E. (2015) Internal barriers of market orientation application. Economics and Management. (14). pp. 535-540.

Tomkinson, R. (2007) Shared services in local government: improving service delivery. Gower (ed.) Aldershot, England; Burlington, VT: Gower.

TRANSLATED (2016) T-Index 2016: the rankings for online markets [Online]. Available from http://www.translated.net/en/languages-that-matter [Accessed: 21 July 2016].

Turle, M. (2010) Shared services: An outline of key contractual issues. Computer Law \& Security Review. 26 (2). pp. 178-184.

Ulbrich, F. (2010) Adopting shared services in a public sector organization M. Janssen (ed.). Transforming Government: People, Process and Policy. 4 (3). pp. 249-265.

Ulrich, D. (1995) Shared Services: From Vogue to Value. Human Resource Planning. 18 (3). pp. 12-23. [Online]. Available from

http://search.ebscohost.com/login.aspx?direct=true \&db=bth\&AN=5667835\&site=eho st-live\%5Cnhttp://www.questia.com/googleScholar.qst?docId=5001655953.

Waddell, D. \& Sohal, A.S. (1998) Resistance: a constructive tool for change management. Management Decision. 36 (8). pp. 543-548.

Walker, R.M., Brewer, G.A., Boyne, G.A. \& Avellaneda, C.N. (2011) Market orientation and public service performance: new public management gone mad? Public administration review. 71 (5). pp. 707-717.

Walsh, G. \& Beatty, S.E. (2007) Customer-based corporate reputation of a service firm: scale development and validation. Journal of the Academy of Marketing Science. 35 (1) p.pp. 127-143.

Walsh, P., McGregor-Lowndes, M., Newton, C.J., McGregor-Lowndes, M. \& Newton, C.J. (2008) Shared services: Lessons from the public and private sectors for the nonprofit sector. Australian Journal of Public Administration. 67 (2). pp. 200-212.

Wang, S., Wang, H., Mary, S. \& Bh, N.S. (2007) Shared services beyond sourcing the back offices: Organizational design. Human Systems Management. 26 (4). pp. 281-290. [Online]. Available from http://iospress.metapress.com/index/u0538g0578325x58.pdf.

Webb, C. (1989) Action research: philosophy, methods and personal experiences. Journal of Advanced Nursing. 14 (5). pp. 403-410. [Online]. DOI: 10.1111/j.13652648.1989.tb01548.x [Accessed: 20 May 2017].

Weeks, W.A., Roberts, J., Chonko, L.B. \& Jones, E. (2004) Organizational readiness for change, individual fear of change, and sales manager performance: An empirical investigation. Journal of Personal Selling \& Sales Management. 24 (1). pp. 7-17. 
Wernerfelt, B. (1984) A Resource based view of the firm. Strategic Management Journal. 5 (2). pp. 171-180.

Williams, R. (2016) Multicollinearity [Online]. Available from http://www3.nd.edu/ rwilliam/ [Accessed: 23 July 2016].

Wilson, A.P. (1970) Educational Innovations in Rural America. Educational Resources Information Center (ERIC).

Yusof, A.F., Miskon, S., Iahad, N.A., Rahman, A.A., Abdullah, N.S., Sim, A.T.H. \& Ahmad, N. (2016) DRIVERS INFLUENCING SHARED SERVICES ADOPTION. Journal of Theoretical and Applied Information Technology. 90 (1). pp. 93-100.

Zuniga, R. \& Murillo, R. (2014) Draining the judiciary bottleneck: A quasi-experiment in improving a government service. Journal of Business Research. 67 (6). pp. 1267-1276. 
APPENDICES 


\section{Appendix A - Examples of cases of problems with SSCs in the public or non-profit sector}

\section{Case 1 - Department for Transport SSC - England}

The Department for Transport in 2004 began to examine the chance of gains from the scale in back-office of the Department and its agencies. Then, SSC was already well proven in the English public practice. They set up the SSC in 2006 to provide HR, payroll, finance, and procurement services to six agencies of the Department for Transport (DfT). Since the implementation, there were serious problems with overall performance and systems. The project was poorly managed, with imposing unrealistic dates, which led to the lack of quality in each partial deployment. The costs of carrying out the SSC jumped from 35 million pounds to more than 113 million. They considered returning to a decentralised model, but in the end chose to privatize the SSC that happened in 2013 (NAO, 2008).

The following is a summary of more information about the DfT SSC deployment initiative (Figure 1).

Figure 1 - Summary of information about the DfT SSC (NAO, 2008).

\begin{tabular}{|l|l|}
\hline Evaluated Factors & Findings \\
\hline $\begin{array}{l}\text { 1. Motivation for choosing the } \\
\text { SSC as a solution }\end{array}$ & Better quality of service, cost reduction and integration \\
\hline 2.Scope & HR, payroll, finance, and procurement \\
\hline 3.Clients & The DfT plus six agencies \\
\hline 4. Estimated benefits & Gross savings of $£ 112.4$ million up to March 2015 \\
\hline 5.Leadership of the program & Politicians of Department \\
\hline 6.SSC model & In-house, centralised SSC \\
\hline 7.Problems highlighted & $\begin{array}{l}\text { Very demanding project schedule; they did not agree on a } \\
\text { common set of business processes; first two agencies not } \\
\text { well prepared for the implementation; poor program } \\
\text { governance and staffing, priorities definition and } \\
\text { implementation strategy }\end{array}$ \\
\hline 8. Main difficulties & $\begin{array}{l}\text { Lack of commitment of the stakeholders; not enough skills } \\
\text { to manage complex projects; political interference }\end{array}$ \\
\hline
\end{tabular}




\begin{tabular}{|l|l|}
\hline Evaluated Factors & Findings \\
\hline 9.Financial loss & f81.1 million of loss \\
\hline 10.Final adopted solution & SSC was outsourced \\
\hline
\end{tabular}

\section{Case 2 - Government of Western Australian SSC}

After many cases of success of SSC in the public sector of the British Commonwealth, the Government of Western Australia implemented the SSC in 2003 for payroll services and administration of the public service with annual benefits of 50 million Australian dollars estimated. The total cost of 80 million ended up being more than 440 million. In 2011, most customers were dissatisfied, and the cost of the SSC services was higher than if the services were performed in government agencies, clients of SSC. The government opted for the rollback of the SSC, which will cost around two billion Australian dollars for the taxpayer (NAO, 2008; NCA, 2015). See Figure 2 below for a summary of information for the Case 2 .

Figure 2 - Summary of information about the Government of Western Australian SSC (NCA, 2015).

\begin{tabular}{|l|l|}
\hline Evaluated Factors & Findings \\
\hline $\begin{array}{l}\text { 1. Motivation for choosing } \\
\text { the SSC as a solution }\end{array}$ & Cost cut of \$50 million per year \\
\hline 2.Scope & Payroll services \\
\hline 3.Clients & Agencies \\
\hline 4.Estimated benefits & \$50 million per year \\
\hline 5. Leadership of the program & Government politicians \\
\hline 6.SSC model & In-house, centralised SSC \\
\hline 7.Problems highlighted & $\begin{array}{l}\text { Costs underestimated, serious errors, unhappy customers, } \\
\text { the \$80 million initial investment turned into more than } \\
\text { \$440 million }\end{array}$ \\
\hline Main difficulties & $\begin{array}{l}\text { Unrealistic Business Case; lack of skills and experience to } \\
\text { manage the complex program; processes and systems not } \\
\text { standardised before the consolidation; did not manage the } \\
\text { change comprehensively and effectively }\end{array}$ \\
\hline 9. Financial loss & Over \$1 billion \\
\hline 10.Final adopted solution & $\begin{array}{l}\text { Rollback. The SSC was discontinued, and each agency has } \\
\text { returned to its payroll service }\end{array}$ \\
\hline
\end{tabular}




\section{Case 3 - Yale University SSC}

There are many American universities that have deployed or are deploying initiatives of SSC (e.g., University of Michigan, Berkeley, University of California, Santa Barbara, Cornell University, University of North Carolina, University of New Hampshire and Yale University), seeking to reduce its operating costs and devote more resources to their main activities related to education.

The Yale University, a private not-for-profit organisation, founded in 1701, adopted an SSC to provide financial, administrative, procurement and HR services, for all its departments aiming at helping to reduce the $\$ 350$ million operating deficit of the university, and share resources. They formed the SSC in January 2010 by consolidating three existing service units. SSC's clients recently have stepped up their complaints about the lack of responsiveness of the SSC managers. They further argue the SSC has not reached its goal of simplifying their lives, but rather, much more complicated (Yale Shared Services, 2011; Yale Daily News, 2012b, 2012a). Next, Figure 3 shows a summary of information about the Yale SSC.

Figure 3 - Summary of information about the Yale SSC (Yale Shared Services, 2011).

\begin{tabular}{|l|l|}
\hline Evaluated Factors & Findings \\
\hline $\begin{array}{c}\text { 1. Motivation for choosing } \\
\text { the SSC as a solution }\end{array}$ & $\begin{array}{l}\text { Cost reduction within a broader operational transformation } \\
\text { program to have more employees in research and education }\end{array}$ \\
\hline 2.Scope & Administrative and financial services, procurement and HR \\
\hline 3.Clients & All university departments \\
\hline 4.Estimated benefits & Not informed \\
\hline $\begin{array}{l}\text { 5.Leadership of the } \\
\text { program }\end{array}$ & Academics and experienced executives \\
\hline 6.SSC model & In-house, centralised SSC \\
\hline 7.Problems highlighted & $\begin{array}{l}\text { The SSC does not meet the needs of individual departments; } \\
\text { customers feel there was imposing a centralised model; lack } \\
\text { of response from SSC managers; impact of SSC in the } \\
\text { departments not properly anticipated; poor SSC staff skills; }\end{array}$ \\
\hline
\end{tabular}




\begin{tabular}{|l|l|}
\hline Evaluated Factors & Findings \\
\hline 8. Main difficulties & $\begin{array}{l}\text { customers do not realize improvements to the quality of } \\
\text { services and do not know the cost cut amount }\end{array}$ \\
\hline 9. Financial loss & $\begin{array}{l}\text { Distance between the program leadership and customers due } \\
\text { to trying to speed up the deployment; lack of sensitivity for } \\
\text { activities that are not provided by SSC, whose resources were } \\
\text { removed from the departments }\end{array}$ \\
\hline 10.Final adopted solution & $\begin{array}{l}\text { The solution had not yet been found, but the SSC will be } \\
\text { maintained, with adjustments to improve the quality of } \\
\text { service }\end{array}$ \\
\hline
\end{tabular}

It is important to underline that, although the focus here is the SSC project failures, there are hundreds of success stories of SSC in Governments and other non-profit organisations, and the purpose here is to highlight some problems to be further investigated through this research. Usually, the public managers seek most of the time the reduction of costs and improvement of the quality of service when they choose to implement an SSC, but the complexity of the organisational transformation required is an important barrier to transpose (Paagman et al., 2015). 


\section{Appendix B - EUSurvey Questionnaire}

\section{Factors Influencing the Performance of Shared Services Centre}

Fields marked with * are mandatory.

Disclaimer

The European Commissian is nat responsuble for the content of questionnstres cragted using the EUSUrvey senice - if remaing the sole responsitwily of the form creafor and manager, The use of EUSumey sember does not imply a recommendation or endorsenent, by the Eumpasn Commission, of the wews expressed within thent

实

Dear respondent,

This survey will take approximately 15 minute to complete. Thank you for taking your time to help improve the public Shared Services Centres (SSC).

The survey aims to identify the extent to which factors such as market orientation, organizational culture, leadership, and partnership impact the performance of public SSCs. The data gathered will be used in the doctoral thesis of Cicero Ferreira at the University of Liverpool. The purpose of this research is to identify improvements to the common models of the public SSC.

By filing in the questionnaire, you express your understanding that your participation is voluntary, and that you are free to withdraw at any time without giving any reason, and without your rights being affected. Moreover, that you understand that confidentiality and anonymity will be maintained, and it will not be possible to identify you in any publications. If deemed necessary, you can contact the University of Liverpool Research Participant Advocate via email: liverpoolethics@ohecampus.com.

If you have any questions or comments, please do not hesitate to contact me:

\section{Cicero Ferreira}

cicero-ferreira@iverpool.ac.uk

Let's begin!

The following seven sections present statements for which you must express your agreement or disagreement based on the Likert scale 1-7 on the right, where 1 is Strongly Disagree and 7 is Strongly Agree. In the last section (number eight), there is one open-ended question and two multiple choice questions. 


\section{SSC MARKET ORIENTATION}

When answering the following questions, please focus on a public SSC organization you know well.

SSC MARKET ORIENTATION

\begin{tabular}{|c|c|c|c|c|c|c|c|}
\hline & $\begin{array}{c}\text { Strongly } \\
\text { Disagree } \\
1\end{array}$ & 2 & 3 & 4 & 5 & 6 & $\begin{array}{c}\text { Strongly } \\
\text { Agree } \\
7\end{array}$ \\
\hline $\begin{array}{l}\text { "Our business } \\
\text { objectives are } \\
\text { driven primarily by } \\
\text { customericitizen } \\
\text { satisfaction. }\end{array}$ & 6 & 0 & 0 & $\theta$ & 6 & 0 & 0 \\
\hline $\begin{array}{l}\text { We constantly } \\
\text { monitor our level } \\
\text { of commitment } \\
\text { and orientation to } \\
\text { serving } \\
\text { customericitizen } \\
\text { needs. }\end{array}$ & 0 & 0 & 0 & $\theta$ & 0 & 0 & 0 \\
\hline $\begin{array}{l}\text { We measure } \\
\text { customericitizen } \\
\text { satisfaction } \\
\text { systematically and } \\
\text { frequently. }\end{array}$ & 0 & 0 & $\theta$ & 6 & 0 & $\theta$ & $\theta$ \\
\hline $\begin{array}{l}\text { "We have routine } \\
\text { or regular } \\
\text { measures of } \\
\text { customericitizen } \\
\text { service. }\end{array}$ & $\Theta$ & 0 & $\theta$ & 6 & 0 & 0 & $\theta$ \\
\hline $\begin{array}{l}\text { "I believe this SSC } \\
\text { exists primarily to } \\
\text { serve } \\
\text { customersvcitizens. }\end{array}$ & $\theta$ & 0 & 0 & 0 & 0 & 0 & 0 \\
\hline $\begin{array}{l}\text { "We poll } \\
\text { end-users at least } \\
\text { once a year to } \\
\text { assess the quality } \\
\text { of our products } \\
\text { and services. }\end{array}$ & 0 & 0 & 0 & 0 & 0 & 0 & 0 \\
\hline
\end{tabular}




\begin{tabular}{l|l|l|l|l|l|} 
"Data on customer & & & & & \\
satisfaction are \\
disseminated at all \\
levels in this \\
business unit on a \\
regular basis.
\end{tabular}

\section{SSC CULTURE}

When answering the following questions, please focus on a public SSC organization you know well.

SSC CULTURE - Quality culture

\begin{tabular}{|c|c|c|c|c|c|c|c|}
\hline & $\begin{array}{c}\text { Strongly } \\
\text { Disagree } \\
1\end{array}$ & 2 & 3 & 4 & 5 & 6 & $\begin{array}{c}\text { Strongly } \\
\text { Agree } \\
7\end{array}$ \\
\hline $\begin{array}{l}\text { 'Continuous } \\
\text { quality } \\
\text { improvement is } \\
\text { part of all } \\
\text { employees' } \\
\text { responsibility } \\
\text { rather than of the } \\
\text { quality } \\
\text { department } \\
\text { abone. }\end{array}$ & 0 & 0 & 0 & 0 & $\theta$ & $\theta$ & 6 \\
\hline $\begin{array}{l}\text { *Our SSC } \\
\text { establishes } \\
\text { long-term goals } \\
\text { related to quality. }\end{array}$ & $\phi$ & 6 & $\theta$ & $\theta$ & $\theta$ & $\theta$ & 0 \\
\hline $\begin{array}{l}\text { "Our SSC } \\
\text { develops an } \\
\text { organization-wide } \\
\text { culture of quality. }\end{array}$ & 0 & $\theta$ & 8 & 0 & $\theta$ & $\theta$ & 6 \\
\hline
\end{tabular}

SSC CULTURE - Service culture

\begin{tabular}{|l|c|c|c|c|c|c|c|}
\hline & $\begin{array}{l}\text { Strongly } \\
\text { Disagree } \\
1\end{array}$ & 2 & 3 & 4 & 5 & 6 & $\begin{array}{c}\text { Strongly } \\
\text { Agree }\end{array}$ \\
\hline $\begin{array}{l}\text {-Service quality } \\
\text { values are } \\
\text { explicitly } \\
\text { addressed and } \\
\text { actively }\end{array}$ & 6 & 0 & 0 & 0 & & & 7 \\
\hline
\end{tabular}




\begin{tabular}{|c|c|c|c|c|c|c|c|}
\hline $\begin{array}{l}\text { promoted within } \\
\text { our } \\
\text { organization. }\end{array}$ & & & & & & & \\
\hline $\begin{array}{l}\text { "Our } \\
\text { organization } \\
\text { promotes a } \\
\text { culture that } \\
\text { emphasizes } \\
\text { service. }\end{array}$ & 0 & 0 & 0 & 0 & 0 & 0 & 0 \\
\hline $\begin{array}{l}\text {-Our } \\
\text { employees are } \\
\text { fully committed } \\
\text { to } \\
\text { customerloitizen } \\
\text { service. }\end{array}$ & 0 & 0 & 0 & 0 & 0 & 0 & 0 \\
\hline
\end{tabular}

SSC CULTURE - Organizational buy-in

\begin{tabular}{|c|c|c|c|c|c|c|c|}
\hline & $\begin{array}{c}\text { Strongly } \\
\text { Disagree } \\
1\end{array}$ & 2 & 3 & 4 & 5 & 6 & $\begin{array}{c}\text { Strongly } \\
\text { Agree } \\
7\end{array}$ \\
\hline $\begin{array}{l}\text { "Across the } \\
\text { organization, } \\
\text { there was a } \\
\text { high level of } \\
\text { "buy in" for } \\
\text { this SSC. }\end{array}$ & 0 & 6 & 0 & 0 & 0 & 6 & 0 \\
\hline $\begin{array}{l}\text { "Our work } \\
\text { group felt } \\
\text { like we were } \\
\text { on our own } \\
\text { in trying to } \\
\text { make the } \\
\text { SSC a } \\
\text { success. }\end{array}$ & $\theta$ & 0 & 0 & 0 & 0 & 0 & 0 \\
\hline $\begin{array}{l}\text { "There was } \\
\text { a general } \\
\text { lack of } \\
\text { support for } \\
\text { the SSC } \\
\text { across our } \\
\text { government } \\
\text { organization. }\end{array}$ & 0 & 0 & 0 & 0 & 0 & 0 & 0 \\
\hline $\begin{array}{l}\text { "There was } \\
\text { a }\end{array}$ & & & & & & & \\
\hline
\end{tabular}




\begin{tabular}{l|l|l|l|l|} 
tremendous & groundswell \\
of support in & & & & \\
the \\
government \\
organization \\
for the SSC.
\end{tabular}

\section{SSC LEADERSHIP}

When answering the following questions, please focus on a public SSC organization you know well.

\section{SSC LEADERSHIP}

\begin{tabular}{|c|c|c|c|c|c|c|c|}
\hline & $\begin{array}{c}\text { Strongly } \\
\text { Disagree } \\
1\end{array}$ & 2 & 3 & 4 & 5 & 6 & $\begin{array}{c}\text { Sirongly } \\
\text { Agree } \\
7\end{array}$ \\
\hline $\begin{array}{l}\text { "Leaders } \\
\text { develop the } \\
\text { mission, vision, } \\
\text { values and } \\
\text { ethics and are } \\
\text { role models for } \\
\text { a culture of } \\
\text { excellence. }\end{array}$ & 0 & $\theta$ & $\theta$ & $\theta$ & $\theta$ & $\theta$ & 6 \\
\hline $\begin{array}{l}\text { "Managers } \\
\text { become } \\
\text { involved in } \\
\text { nunning the } \\
\text { SSC as a set } \\
\text { of interrelated } \\
\text { processes, all } \\
\text { of them } \\
\text { responsible for } \\
\text { quality. }\end{array}$ & 0 & $\theta$ & $\theta$ & $\theta$ & $\theta$ & $\theta$ & 0 \\
\hline $\begin{array}{l}\text { "Managers } \\
\text { ensure that } \\
\text { employees are } \\
\text { capable of } \\
\text { taking } \\
\text { initiatives and } \\
\text { assimilating } \\
\text { better ways of } \\
\text { doing their } \\
\text { jobs. }\end{array}$ & $\Theta$ & 0 & 0 & 0 & 0 & 6 & 0 \\
\hline & & & & & & & \\
\hline
\end{tabular}




\begin{tabular}{|c|c|c|c|c|c|c|c|}
\hline $\begin{array}{l}\text { "Leaders } \\
\text { inferact with } \\
\text { cusiomers, } \\
\text { partners and } \\
\text { representatives } \\
\text { of society. }\end{array}$ & 0 & 0 & 0 & 0 & 0 & 0 & 0 \\
\hline $\begin{array}{l}\text { "Managers } \\
\text { behave in a } \\
\text { way that allows } \\
\text { the integration } \\
\text { and } \\
\text { mobilization of } \\
\text { members of a } \\
\text { team. }\end{array}$ & 0 & $\theta$ & 0 & $\theta$ & 0 & 0 & 0 \\
\hline $\begin{array}{l}\text { "Leaders } \\
\text { identify and } \\
\text { champion } \\
\text { organizational } \\
\text { change. }\end{array}$ & 0 & $\theta$ & $\theta$ & 0 & 0 & 0 & 0 \\
\hline $\begin{array}{l}\text { "Managers } \\
\text { continuousty } \\
\text { acquire and } \\
\text { update } \\
\text { knowledge that } \\
\text { is valuable for } \\
\text { the } \\
\text { organization. }\end{array}$ & 0 & 0 & 0 & 0 & 0 & 0 & 0 \\
\hline $\begin{array}{l}\text { "Managers act } \\
\text { in a way that } \\
\text { makes it easier. } \\
\text { for employees } \\
\text { to accept } \\
\text { proposed } \\
\text { changes } \\
\text { voluntarity. }\end{array}$ & 0 & 0 & 0 & 0 & 0 & 0 & 0 \\
\hline
\end{tabular}

\section{SSC RESOURCES}

When answering the following questions, please focus on a public SSC organization you know well.

SSC RESOURCES - Financial resources

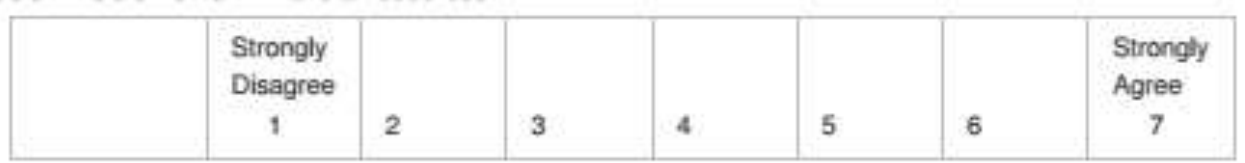




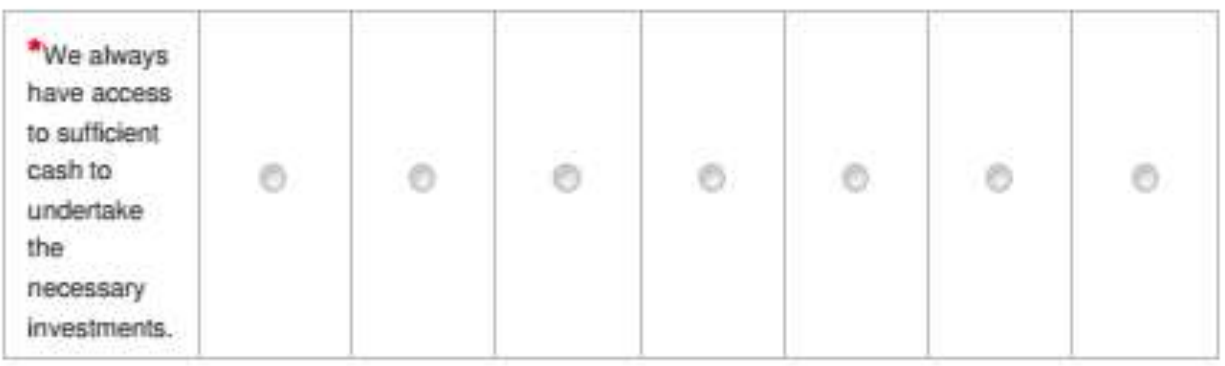

SSC RESOURCES - Physical resources

\begin{tabular}{|l|c|c|c|c|c|c|c|}
\hline & $\begin{array}{c}\text { Strongly } \\
\text { Disagree } \\
1\end{array}$ & 2 & 3 & 4 & 5 & 6 & $\begin{array}{c}\text { Strongly } \\
\text { Agree }\end{array}$ \\
\hline $\begin{array}{l}\text { * Our locations } \\
\text { are excellent } \\
\text { with respect to } \\
\text { customericitizen } \\
\text { needs. }\end{array}$ & 0 & 6 & 0 & 0 & 0 & 0 & 0 \\
\hline $\begin{array}{l}\text { *Our equipment } \\
\text { is excellent for } \\
\text { our purposes. }\end{array}$ & 0 & 0 & 0 & 0 & 0 & 0 & 0 \\
\hline
\end{tabular}

SSC RESOURCES - Human Resources

\begin{tabular}{|c|c|c|c|c|c|c|c|}
\hline & $\begin{array}{c}\text { Strongly } \\
\text { Disagree } \\
1\end{array}$ & 2 & 3 & 4 & 5 & 6 & $\begin{array}{c}\text { Strongly } \\
\text { Agree } \\
7\end{array}$ \\
\hline $\begin{array}{l}\text { "The } \\
\text { knowledge, } \\
\text { skills and } \\
\text { capabilities of } \\
\text { our } \\
\text { employees } \\
\text { are superior } \\
\text { to those of } \\
\text { similar } \\
\text { organizations. }\end{array}$ & 0 & 0 & 6 & 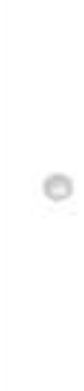 & 0 & 0 & 0 \\
\hline $\begin{array}{l}\text { *The skills } \\
\text { and } \\
\text { capabilities of } \\
\text { our top } \\
\text { management } \\
\text { team are }\end{array}$ & 0 & 0 & 0 & 0 & $\theta$ & 0 & 0 \\
\hline
\end{tabular}




\begin{tabular}{|c|c|c|c|c|c|c|c|}
\hline $\begin{array}{l}\text { superior to } \\
\text { that of similar } \\
\text { organizations. }\end{array}$ & & & & & & & \\
\hline $\begin{array}{l}\text { "We can } \\
\text { attract/recruit } \\
\text { qualitied } \\
\text { employees } \\
\text { more easily } \\
\text { than similar } \\
\text { organizations. }\end{array}$ & 0 & 0 & 0 & 0 & 0 & 0 & 0 \\
\hline
\end{tabular}

SSC RESOURCES - Organizational resources, skills, and competencies

\begin{tabular}{|l|c|c|c|c|c|c|c|}
\hline & $\begin{array}{c}\text { Strongly } \\
\text { Disagree } \\
1\end{array}$ & 2 & 3 & 4 & 5 & 6 & $\begin{array}{c}\text { Strongly } \\
\text { Agree } \\
7\end{array}$ \\
\hline $\begin{array}{l}\text { *Interaction with } \\
\text { customers/citizens } \\
\text { gives direction to } \\
\text { the innovation } \\
\text { process. }\end{array}$ & 0 & 0 & 0 & 0 & 0 & 0 & 0 \\
\hline $\begin{array}{l}\text { *We scan the } \\
\text { environment } \\
\text { constantly for new } \\
\text { oppontunities. }\end{array}$ & 0 & 0 & 0 & 0 & 0 & 0 & 0 \\
\hline $\begin{array}{l}\text { *Cross-functionat } \\
\text { groups work } \\
\text { together to } \\
\text { innovate. }\end{array}$ & 0 & 0 & 0 & 0 & 0 & 0 & 0 \\
\hline
\end{tabular}

SSC RESOURCES - Service specitic resources, skills, and competencies

\begin{tabular}{|l|c|c|c|c|c|c|c|}
\hline & $\begin{array}{l}\text { Strongly } \\
\text { Disagree } \\
1\end{array}$ & 2 & 3 & 4 & 5 & 6 & $\begin{array}{c}\text { Strongly } \\
\text { Agree } \\
7\end{array}$ \\
\hline $\begin{array}{l}\text { We are very } \\
\text { rellable and } \\
\text { precise in } \\
\text { delivering cur } \\
\text { services. }\end{array}$ & 0 & 0 & 0 & 0 & 0 & 0 & 0 \\
\hline $\begin{array}{l}\text { "We are ready to } \\
\text { help and serve } \\
\text { our } \\
\text { customersicitizens } \\
\text { swittly. }\end{array}$ & 0 & 0 & 0 & 0 & 0 & 0 & 0 \\
\hline
\end{tabular}




\begin{tabular}{|c|c|c|c|c|c|c|c|}
\hline $\begin{array}{l}\text { We measure } \\
\text { customericitizen } \\
\text { satisfaction } \\
\text { regularty. }\end{array}$ & $\theta$ & 0 & $\theta$ & 6 & 0 & 0 & $\theta$ \\
\hline
\end{tabular}

\section{SSC READINESS FOR CHANGE}

When answering the following questions, please focus on a public SSC organization you know well.

SSC READINESS FOR CHANGE - Commitment of senior management to the change

\begin{tabular}{|c|c|c|c|c|c|c|c|}
\hline & $\begin{array}{c}\text { Strongly } \\
\text { Disagree } \\
1\end{array}$ & 2 & 3 & 4 & 5 & 6 & $\begin{array}{c}\text { Strongly } \\
\text { Agree } \\
7\end{array}$ \\
\hline $\begin{array}{l}\text { "Senior } \\
\text { management } \\
\text { is decisive } \\
\text { with respect } \\
\text { to } \\
\text { organizational } \\
\text { goals, } \\
\text { priorities and } \\
\text { strategies } \\
\text { conceming } \\
\text { the change. }\end{array}$ & 0 & 0 & 0 & 0 & $\theta$ & 0 & 0 \\
\hline $\begin{array}{l}\text { "Leaders } \\
\text { themselves } \\
\text { have bought } \\
\text { into the } \\
\text { change and } \\
\text { promote it by } \\
\text { behaving in a } \\
\text { manner } \\
\text { consistent } \\
\text { with the } \\
\text { change. }\end{array}$ & $\theta$ & 0 & 0 & 0 & 0 & $\theta$ & 0 \\
\hline $\begin{array}{l}\text { *There is a } \\
\text { champion of } \\
\text { change at the } \\
\text { most senior } \\
\text { level of the } \\
\text { organization, }\end{array}$ & 0 & $\theta$ & 0 & $\theta$ & 0 & 0 & 0 \\
\hline
\end{tabular}

SSC READINESS FOR CHANGE - Competence of change agents 


\begin{tabular}{|c|c|c|c|c|c|c|c|}
\hline & $\begin{array}{l}\text { Strongly } \\
\text { Disagree } \\
1\end{array}$ & 2 & 3 & 4 & 5 & 6 & $\begin{array}{c}\text { Strongly } \\
\text { Agree } \\
7\end{array}$ \\
\hline $\begin{array}{l}\text { "Change } \\
\text { agents have } \\
\text { done } \\
\text { research to } \\
\text { select the } \\
\text { right type of } \\
\text { change that } \\
\text { addresses } \\
\text { the } \\
\text { underying } \\
\text { causes of } \\
\text { organizational } \\
\text { problems } \\
\text { rather than } \\
\text { just } \\
\text { symmptoms. }\end{array}$ & 0 & 0 & 0 & 0 & 0 & 0 & 0 \\
\hline $\begin{array}{l}\text { "Change } \\
\text { agents are } \\
\text { competent to } \\
\text { answer } \\
\text { employee } \\
\text { questions } \\
\text { about the } \\
\text { change. }\end{array}$ & 0 & 0 & 0 & 0 & 0 & 0 & 0 \\
\hline
\end{tabular}

SSC READINESS FOR CHANGE - Support of immediate manager

\begin{tabular}{|c|c|c|c|c|c|c|c|}
\hline & $\begin{array}{c}\text { Strongly } \\
\text { Disagree } \\
1\end{array}$ & 2 & 3 & 4 & 5 & 6 & $\begin{array}{c}\text { Strongly } \\
\text { Agree } \\
7\end{array}$ \\
\hline $\begin{array}{l}\text { Managers } \\
\text { are held } \\
\text { accountable } \\
\text { for passing } \\
\text { information } \\
\text { on the } \\
\text { change io } \\
\text { their staft. }\end{array}$ & 0 & 0 & 0 & $\theta$ & 0 & 0 & 0 \\
\hline $\begin{array}{l}\text { "Managers } \\
\text { acknowledge } \\
\text { the impact } \\
\text { the change } \\
\text { may have on } \\
\text { their staft. }\end{array}$ & $\theta$ & 0 & 0 & 6 & 0 & 6 & 0 \\
\hline
\end{tabular}




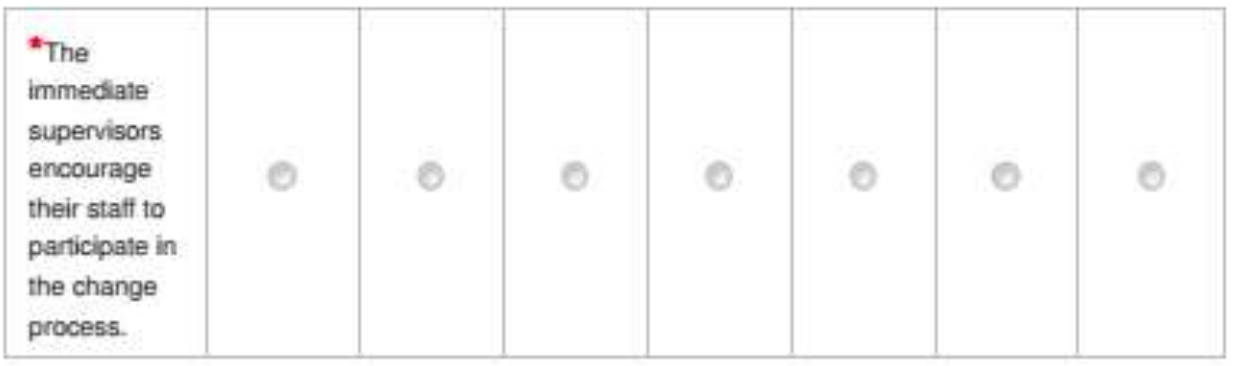

SSC READINESS FOR CHANGE - Adverse impact of the change on work

\begin{tabular}{|c|c|c|c|c|c|c|c|}
\hline & $\begin{array}{c}\text { Strongly } \\
\text { Disagree } \\
1\end{array}$ & 2 & 3 & 4 & 5 & 6 & $\begin{array}{c}\text { Strongly } \\
\text { Agree } \\
7\end{array}$ \\
\hline $\begin{array}{l}\text { "Workdoads } \\
\text { do not } \\
\text { permit } \\
\text { people to } \\
\text { get involved } \\
\text { in the } \\
\text { change } \\
\text { initiatives. }\end{array}$ & 0 & 0 & 0 & 0 & 0 & 0 & 0 \\
\hline $\begin{array}{l}\text { "People are } \\
\text { discouraged } \\
\text { trom saying } \\
\text { 'no' to work } \\
\text { - even } \\
\text { when the } \\
\text { assigned } \\
\text { task is not a } \\
\text { priority. }\end{array}$ & 0 & 6 & 0 & 0 & 0 & 6 & 0 \\
\hline
\end{tabular}

\section{SSC EXCELLENCE}

When answering the following questions, please focus on a public SSC organization you know well.

SSC EXCELLENCE - Excellence

\begin{tabular}{|c|c|c|c|c|c|c|c|}
\hline & $\begin{array}{c}\text { Strongly } \\
\text { Disagree } \\
1\end{array}$ & 2 & 3 & 4 & 5 & 6 & $\begin{array}{c}\text { Strongly } \\
\text { Agree } \\
7\end{array}$ \\
\hline $\begin{array}{l}\text { "The organizatio } \\
\text { instils a value } \\
\text { system in all its } \\
\text { employees. }\end{array}$ & 0 & 0 & 6 & $\theta$ & 6 & 0 & 0 \\
\hline & & & & & & & \\
\hline
\end{tabular}




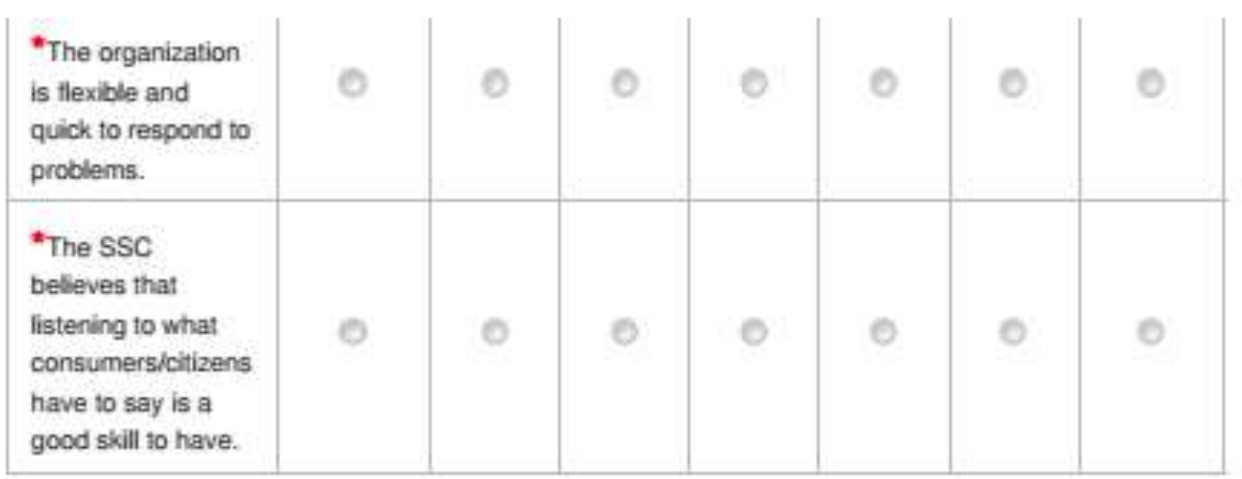

SSC EXCELLENCE - Service quality

\begin{tabular}{|l|l|l|l|l|l|l|l|}
\hline & $\begin{array}{c}\text { Strongly } \\
\text { Disagree } \\
1\end{array}$ & 2 & 3 & 4 & 5 & 6 & $\begin{array}{c}\text { Strongly } \\
\text { Agree } \\
7\end{array}$ \\
\hline $\begin{array}{l}\text { *The SSC offers } \\
\text { the greatest quality } \\
\text { in every service. }\end{array}$ & 0 & 0 & 0 & 0 & 0 & 0 & 0 \\
\hline $\begin{array}{l}\text { "The employees } \\
\text { are trained to } \\
\text { provide correctly } \\
\text { the services to } \\
\text { customers/citizens. }\end{array}$ & 0 & 0 & 0 & 0 & 0 & 0 & 0 \\
\hline $\begin{array}{l}\text { we have reduced } \\
\text { the number of } \\
\text { complaints and } \\
\text { conflicts. }\end{array}$ & 0 & 0 & 0 & 0 & 0 & 0 & 0 \\
\hline
\end{tabular}

SSC EXCELLENCE - Benchmarking

\begin{tabular}{|l|c|l|l|l|l|l|l|}
\hline & $\begin{array}{c}\text { Strongly } \\
\text { Disagree } \\
1\end{array}$ & 2 & 3 & 4 & 5 & 6 & $\begin{array}{c}\text { Strongly } \\
\text { Agree } \\
7\end{array}$ \\
\hline $\begin{array}{l}\text { *Our SSC } \\
\text { has engaged } \\
\text { in extensive } \\
\text { benchmarking } \\
\text { of other } \\
\text { companies' } \\
\text { business } \\
\text { processes in } \\
\text { other } \\
\text { industrites. }\end{array}$ & 0 & 0 & 0 & 0 & 0 & 0 & 0 \\
\hline & & & & & & & \\
\hline
\end{tabular}




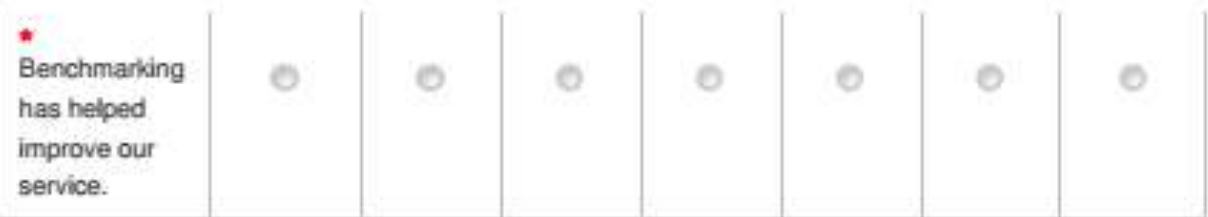

SSC EXCELLENCE - Service standards

\begin{tabular}{|c|c|c|c|c|c|c|c|}
\hline & $\begin{array}{c}\text { Strongly } \\
\text { Disagree } \\
1\end{array}$ & 2 & 3 & 4 & 5 & 6 & $\begin{array}{c}\text { Strongly } \\
\text { Agree } \\
7\end{array}$ \\
\hline $\begin{array}{l}\text { "Our SSC has } \\
\text { established service } \\
\text { standards based } \\
\text { on researched } \\
\text { cuslomericitizen } \\
\text { needs. }\end{array}$ & 0 & 0 & 0 & 0 & 0 & 0 & 0 \\
\hline $\begin{array}{l}\text { "Service } \\
\text { standards are } \\
\text { visible to both } \\
\text { employees and } \\
\text { customersicitizens. }\end{array}$ & 0 & 0 & 0 & 0 & 0 & 0 & 0 \\
\hline $\begin{array}{l}\text { "We have } \\
\text { established service } \\
\text { standards based } \\
\text { on external } \\
\text { benchmarks of } \\
\text { similar } \\
\text { organizations' } \\
\text { periormance. }\end{array}$ & 0 & 0 & 0 & $\theta$ & 0 & 0 & 0 \\
\hline
\end{tabular}

SSC EXCELLENCE - Value orientation

\begin{tabular}{|c|c|c|c|c|c|c|c|}
\hline & $\begin{array}{c}\text { Strongly } \\
\text { Disagree } \\
1\end{array}$ & 2 & 3 & 4 & 5 & 6 & $\begin{array}{c}\text { Strongly } \\
\text { Agree } \\
7\end{array}$ \\
\hline $\begin{array}{l}\text { "Providing } \\
\text { high-value } \\
\text { services is a } \\
\text { major focus } \\
\text { of managerial } \\
\text { attention in } \\
\text { our } \\
\text { organisation. }\end{array}$ & 6 & 0 & 0 & 0 & $\theta$ & $\theta$ & 0 \\
\hline $\begin{array}{l}\text { We have } \\
\text { programs in }\end{array}$ & & & & & & & \\
\hline
\end{tabular}




\begin{tabular}{|c|c|c|c|c|c|c|c|}
\hline $\begin{array}{l}\text { place io } \\
\text { tackde all } \\
\text { activities that } \\
\text { do not add } \\
\text { value to the } \\
\text { customer. }\end{array}$ & 0 & 0 & 0 & 0 & 0 & 0 & 0 \\
\hline $\begin{array}{l}\text { "Exoeptional } \\
\text { value is } \\
\text { created in } \\
\text { our } \\
\text { organisation } \\
\text { through a } \\
\text { combination } \\
\text { of quality. } \\
\text { service } \\
\text { design and } \\
\text { cost } \\
\text { management. }\end{array}$ & 8 & 0 & 0 & 0 & 0 & 0 & 0 \\
\hline
\end{tabular}

\section{SSC PERFORMANCE}

When answering the following questions, please focus on a public SSC organization you know well.

SSC PERFORMANCE - Goal orientation

\begin{tabular}{|c|c|c|c|c|c|c|c|}
\hline & $\begin{array}{c}\text { Strongly } \\
\text { Disagree } \\
1\end{array}$ & 2 & 3 & 4 & 5 & 6 & $\begin{array}{c}\text { Strongly } \\
\text { Agree } \\
7\end{array}$ \\
\hline $\begin{array}{l}\text { "The } \\
\text { programs } \\
\text { and } \\
\text { departments } \\
\text { are guided } \\
\text { by goals and } \\
\text { objectives. }\end{array}$ & 0 & 0 & 0 & 0 & 0 & 0 & 0 \\
\hline $\begin{array}{l}\text { The } \\
\text { programs } \\
\text { and } \\
\text { departments } \\
\text { clearty } \\
\text { communicate } \\
\text { strategies for } \\
\text { achieving } \\
\text { objectives. }\end{array}$ & $\theta$ & 0 & $\Theta$ & 0 & 0 & $\theta$ & $\theta$ \\
\hline
\end{tabular}

SSC PERFORMANCE - Measurement system 


\begin{tabular}{|l|c|l|l|l|l|l|l|}
\hline & $\begin{array}{c}\text { Strongly } \\
\text { Disagree } \\
1\end{array}$ & 2 & 3 & 4 & 5 & 6 & $\begin{array}{c}\text { Strongly } \\
\text { Agree } \\
7\end{array}$ \\
\hline $\begin{array}{l}\text { *Our metics } \\
\text { capture what } \\
\text { is } \\
\text { strategically } \\
\text { important for } \\
\text { measuring } \\
\text { customer } \\
\text { satistaction. }\end{array}$ & 0 & 0 & 0 & 0 & 0 & 0 & 0 \\
\hline $\begin{array}{l}\text { Non-financial } \\
\text { pertormance } \\
\text { measures } \\
\text { such as } \\
\text { employee } \\
\text { satisfaction } \\
\text { and } \\
\text { customer } \\
\text { satistaction } \\
\text { are routinely } \\
\text { used to } \\
\text { evaluate } \\
\text { success. }\end{array}$ & 0 & 0 & 0 & 0 & 0 & & \\
\hline
\end{tabular}

SSC PERFORMANCE - Resources for performance measurement

\begin{tabular}{|c|c|c|c|c|c|c|c|}
\hline & $\begin{array}{c}\text { Strongly } \\
\text { Disagree } \\
1\end{array}$ & 2 & 3 & 4 & 5 & 6 & $\begin{array}{c}\text { Strongly } \\
\text { Agree } \\
7\end{array}$ \\
\hline $\begin{array}{l}\text { "The } \\
\text { organization } \\
\text { has } \\
\text { cominitied } \\
\text { resources } \\
\text { (time, people, } \\
\text { and money) } \\
\text { to be used in } \\
\text { the } \\
\text { measurement } \\
\text { of program. } \\
\text { perlormance. }\end{array}$ & 0 & 0 & 0 & 0 & 0 & 0 & 0 \\
\hline
\end{tabular}

SSC PERFORMANCE - Pertormance 


\begin{tabular}{|c|c|c|c|c|c|c|c|}
\hline & $\begin{array}{c}\text { Strongly } \\
\text { Disagree } \\
1\end{array}$ & 2 & 3 & 4 & 5 & 6 & $\begin{array}{c}\text { Strongly } \\
\text { Agree } \\
7\end{array}$ \\
\hline $\begin{array}{l}\text { "The retum } \\
\text { on } \\
\text { investment } \\
\text { in the SSC } \\
\text { services } \\
\text { achieved or } \\
\text { exceeded } \\
\text { their } \\
\text { objectives. }\end{array}$ & 0 & 0 & 0 & 0 & 0 & 0 & 0 \\
\hline $\begin{array}{l}\text { "The } \\
\text { systems } \\
\text { (hardware, } \\
\text { software, } \\
\text { delivery } \\
\text { systems) } \\
\text { developed } \\
\text { to launch } \\
\text { the SSC } \\
\text { services } \\
\text { provided a } \\
\text { basis for a } \\
\text { better } \\
\text { introduction } \\
\text { of services } \\
\text { in the } \\
\text { future. }\end{array}$ & 0 & 0 & 0 & $\theta$ & 0 & 0 & 6 \\
\hline $\begin{array}{l}\text { "The SSC } \\
\text { services } \\
\text { increased } \\
\text { motivation } \\
\text { and } \\
\text { productivity } \\
\text { among } \\
\text { employees. }\end{array}$ & 0 & 0 & 0 & 0 & 0 & 0 & 0 \\
\hline $\begin{array}{l}\text { "The SSC } \\
\text { services } \\
\text { were more } \\
\text { reliable, } \\
\text { accurate } \\
\text { and of } \\
\text { consistent } \\
\text { quality. }\end{array}$ & 0 & 0 & 0 & $\theta$ & 0 & 0 & 6 \\
\hline
\end{tabular}


SSC PERFORMANCE - Society results

\begin{tabular}{|c|c|c|c|c|c|c|c|}
\hline & $\begin{array}{c}\text { Strongly } \\
\text { Disagree } \\
1\end{array}$ & 2 & 3 & 4 & 5 & 6 & $\begin{array}{c}\text { Strongly } \\
\text { Agree } \\
7\end{array}$ \\
\hline $\begin{array}{l}\text { "The SSC } \\
\text { has a } \\
\text { positive } \\
\text { impact in } \\
\text { society. }\end{array}$ & 0 & 0 & 0 & 0 & 0 & 0 & 0 \\
\hline
\end{tabular}

\section{FINAL QUESTIONS}

1) Do you believe there are significant differences between private sector and public sector SSC models?

If yes, what are the main differences?

If not, why not?

*2) The SSC in your organization is considered mostly as:

6. A new management model

(6) An operational solution

An organizational solution

A cut cost altemative

Other (please, specify):

If you chose Other above, please, specity:

«3) As for the SSC Model you are evaluating: 
The model of our SSC IS VERY SIMILAR to the SSC of the private sector, and this is NOT A PROBLEM at all.

The model of our SSC IS VERY SIMILAR to the SSC of the private sector, and this BRINGS MAJOR PROBLEMS to our operation.

The model of our SSC IS VERY SIMILAR to the SSC of the private sector, and this BRINGS MINOR PROBLEMS to our operations.

The model of our SSC IS NOT SIMILAR to the SSC of the private sector (it was designed specifically for the public sector).

Other (please, specify):

If you chose Other above, please, specity:

\section{SSC INFORMATION AND RESPONDENT PROFILE}

*Evaluated Organization (SSC) name:

*Country where the Organization (SSC) is located:

\# Number of SSC employees:

U. Up to 20

$21-50$

C $51-100$

O $101-200$

201 and above

* In your opinion, at which of the following stages of development is your SSC:

Visioning (design stage)

Launching (deployment)

Growing (deployed and in development)

Transforming/Sustaining (enhancement/changing)

Other (please, specity):

If you chose Other above, please, specily: 
¿For how many years has this SSC been running (approximately)?

Less than 2

O. 2 - 5

C. $6-10$

More than 10

* The customers of this SSC are (you can choose more than one):

7. Citizens

Departments/agencies

$\square$ Other (please, specify):

If you chose Other above, please, specify:

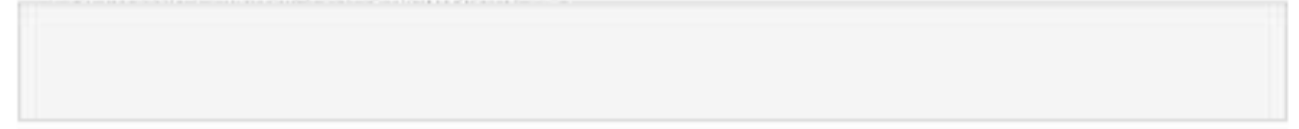

\pm Your position in this organization:
General Manager
(6) Manager
(1) Specialist
Coordinator
3 Anasyst
Other (please, specify):

If you chose Other above, please, specity:

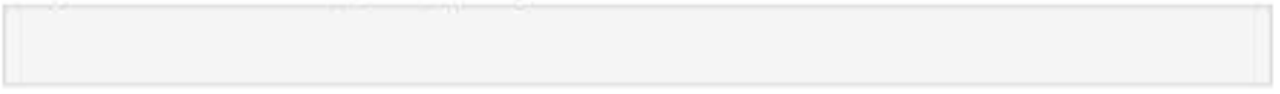

* Taking into account your SSC experience, do you consider yourself as:
An SSC expert
Q SSC experienced
- SSC knowledgeable
Other (please, specify)

If you chose Other above, please, specify:

* How many years of experience in SSC do you have?
U. Up to 2
3-5
6- 10
More than 10 
* How many years of experience in the public sector do you have?
Up to 2
$3-5$
6. $6-10$
C. More than 10

^How many years of experience in the private sector do you have (if any)?

No experience in the private sector

U. Up to 2

(3) $3-5$

$6-10$

Q. More than 10

Once more, thank you for participating in this study on the public Shared Services Centre. Your participation provides valuable data that could help improve this service and is greatly appreciated.

If you have any questions or comments, please do not hesitate to contact me.

Cicero Ferreira

cicero.ferreira@irverpool.ac.uk 


\section{Appendix C - Ethical Committee Approval}

Below the copy of the ethical protocol approval to run the research.

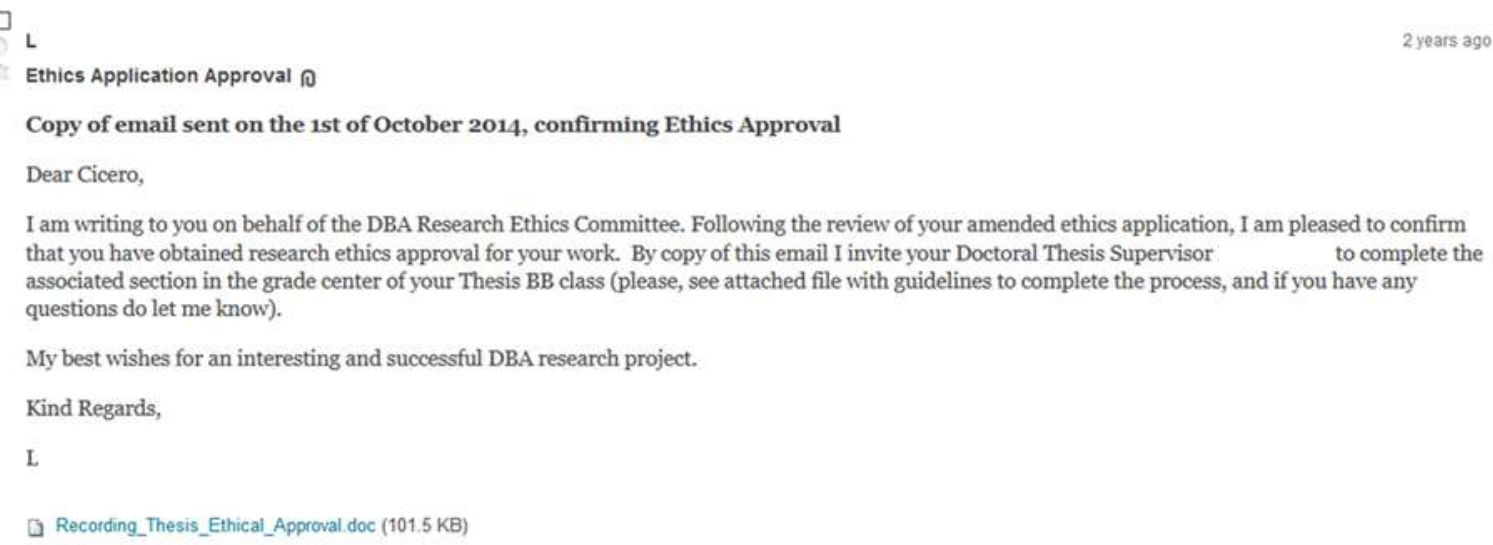

Copy of email sent on the 1st of October 2014, confirming Ethics Approval

Dear Cicero,

I am writing to you on behalf of the DBA Research Ethics Committee. Following the review of your amended ethics application, I am pleased to confirm that you have obtained research ethics approval for your work. By copy of this email I invite your Doctoral Thesis Supervisor. to complete the associated section in the grade center of your Thesis BB class (please, see attached file with guidelines to complete the process, and if you have any questions do let me know)

My best wishes for an interesting and successful DBA research project.

Kind Regards,

[. Recording_Thesis_Ethical_Approval.doc (101.5 KB) 


\section{Appendix D - Example of Emails to the Action Learning Set}

\section{Example 1}

Subject: Support for my doctorate - Action Learning Set - Additional Information

Dear participants of the Action Learning Set,

As agreed, below are the additional information for your participation in the Action Learning Set of my doctorate.

As you know, the purpose of your participation is helping me to improve the analyses, interpretation, preliminary findings, recommendations and practical application of my research. The main goal of this reflection and discussion is to identify the extent to which my research's findings can be used in an SSC organisation and bring actionable knowledge. Additionally, the results of the research will be discussed to identify if they have any contribution to the development of the professional and academic knowledge.

During the planned action, the learner is assisted by the participants of the action learning set. The participants contribute with their comments, criticism and suggestions for ways and ideas. I am attaching to this e-mail an article about action learning sets if you want to explore a little more the theme.

Our Action Learning Set is composed of 14 experts in public service and/or shared services centre, from Brazil, Canada, the United Kingdom and the United States.

At this moment, I would like to ask if you authorise the disclosure of your name in my thesis. If not, I will completely understand.

In the first week of September, I will develop and send to you open-ended questions based on my preliminary findings for obtaining your help to improve them.

Once more, thank you for your help. I am at your disposal for any clarification, suggestion or comment.

\section{Sincerely,}

Cicero Ferreira

E-mail:

Mobile: 
Planning for the Action Learning Set (ALS) work

\begin{tabular}{|l|l|}
\hline $\begin{array}{l}\text { The first half of } \\
\text { August/2016 }\end{array}$ & $\begin{array}{l}\text { The ALS participants will receive instructions regarding } \\
\text { the course of action. }\end{array}$ \\
\hline $\begin{array}{l}\text { The first week of } \\
\text { September } / 16\end{array}$ & $\begin{array}{l}\text { The first round of assessment and suggestions - The } \\
\text { ALS participants will receive by email my analyses and } \\
\text { preliminary research results. }\end{array}$ \\
\hline $\begin{array}{l}\text { The second week of } \\
\text { September/16 }\end{array}$ & $\begin{array}{l}\text { The participants of the ALS will send by email their } \\
\text { comments and suggestions. }\end{array}$ \\
\hline $\begin{array}{l}\text { The third week of } \\
\text { September /16 }\end{array}$ & $\begin{array}{l}\text { The comments and suggestions are consolidated and } \\
\text { incorporated into the thesis. }\end{array}$ \\
\hline $\begin{array}{l}\text { The fourth week of } \\
\text { September /16 }\end{array}$ & $\begin{array}{l}\text { The second round of assessment and suggestions - } \\
\text { analyses and improved results are forwarded to the ALS } \\
\text { participants for a final review. }\end{array}$ \\
\hline $\begin{array}{l}\text { The fourth week of } \\
\text { September } / 16\end{array}$ & $\begin{array}{l}\text { The participants of the ALS will send by email their final } \\
\text { comments and suggestions. }\end{array}$ \\
\hline $\begin{array}{l}\text { The fourth week of } \\
\text { September } / 16\end{array}$ & $\begin{array}{l}\text { Final comments and suggestions are incorporated into } \\
\text { the thesis. }\end{array}$ \\
\hline
\end{tabular}

\section{Example 2}

Subject: Support for my doctorate - Action Learning Set - First round of discussion Dear participants of the Action Learning Set,

Starting our process of discussion, I present in the document Key Findings enclosed the main findings of the qualitative and quantitative parts of the survey about Shared Services Centre, carried out with 146 respondents from countries such as Brazil, Canada, UK, and the USA. Following each finding, I address open-ended questions to you, the answers to which will help me to check if such findings are relevant and workable in your opinion.

Could you please, read the Key Findings document, reply to the open-ended questions, and send it back to me until September 17? Upon receiving the replies, I will consolidate them, interpret the results and submit them to you for our second and final round of discussion.

Just to recall, the main goal of this reflection and discussion is to identify the extent to which these research's findings can be used in an SSC organisation and bring actionable knowledge.

Thinking of your time, I have summarised the information you will need in the Key findings document. However, for those who want more detail, I have attached the chapters of my thesis Exploratory Qualitative Research, and Data Analysis and Results in full, with the survey context, response details and my complete interpretation for each finding. 
Note: The speakers of Portuguese can answer the questions in Portuguese if preferred. I look forward to your comments. Your help is highly appreciated!

Best regards,

Cicero Ferreira

Email:

\section{1) Key findings of the qualitative part of the survey}

Eighty-six respondents (59\%) understand there are significant differences between the SSCs of the private and public sectors. The responses of this group of respondents were analysed and grouped into five categories listed below, each category with its open-ended questions the following.

Category 01 Private Sector SSC as a service unit - This category represents the set of responses from those who believe that the SSCs from the industry offer services with better quality, better performance, more effectiveness, excellence, and that they are more oriented to customers, innovative and flexible.

My interpretation

A summarized preliminary interpretation, in the light of the theory, is that the starting point for the design of the SSC operation in the private sector tends to be more 'outside in', that is, tends to start at the clients' view, and the attributes that lead to satisfaction with the service (see Table 5.2 attached) tend to be adopted more efficiently by the private SSCs.

Open-ended questions

Do you agree with this interpretation? If not, why not?

In practical terms, what could be done to lead the public SSC to be comparatively more efficient?

Category 02 Public Sector SSC as a (local) public agency - This category includes respondents who consider that the fact that the SSCs of governments having to meet local priorities, provide the services prescribed by law and as they are of compulsory use of its clients (which cannot receive the service from another provider), makes these SSCs comparatively less competitive.

My interpretation 
A summarized preliminary interpretation, in the light of the theory, is that the correct definition of SSC's mission (considering the best use of public money and the outside in design discussed in the previous category), as well as the entrepreneurial attitude of leadership, can soften the negative impact that the bureaucracy and the legislation provoke in the effectiveness of the public SSCs.

Open-ended questions

Do you agree with this interpretation? If not, why not?

In practical terms, what could be done to lead the public SSC to be, comparatively, more competitive?

Category 03 Private Sector SSC as a Business Unit - This category brings together those respondents who claim that the private sector SSCs feature better overall quality because they are conducted as a business, profit-oriented and can fund more easily, especially in times of crisis.

\section{My interpretation}

A summarized preliminary interpretation, in the light of the theory, is that the SSCs of the public sector also can adopt the charging for the offered services and use the resources obtained in the maintenance of services with high level of quality, working as a public agency or in another legal configuration, as a public-private partnership, for example. Another essential factor for this, is the formation of a culture specific for the SSC, focused on excellence and best practices in services with inter-sector benchmarks established, whenever the type of service offered allows.

Open-ended questions

Do you agree with this interpretation? If not, why not?

In practical terms, what could be done to lead the public SSC to be run 'as a business?'

Category 04 Public Sector SSC and the public servant dilemma-Respondents in this category attribute the comparatively lower performance of SSCs of the public sector to the human factor, as the absence of meritocracy as criteria for pay and promotion; the fact that many public SSCs cannot choose their staff; job stability as a sponsor of 
accommodation and not encouraging the continued development; a higher turnover of staff in the SSCs, which reduces the average level of competency of employees and their condition to provide a better service; and a minor commitment of the public officials pro-excellence of service.

\section{My interpretation}

A summarized preliminary interpretation, in the light of the theory, is that since these causes are difficult to change in the public sector, the management theory offers alternatives and best practices in areas of study as motivation, leadership, teamwork and organisational culture as possible remedies for overcoming these barriers to quality of service.

\section{Open-ended questions}

Do you agree with this interpretation? If not, why not?

In practical terms, what could be done to lead the public SSC to raise the quality of service?'

Category 05 Public Sector SSC and the political environment - This category is formed by the comments that affirm that the political interference affects the performance of the public SSCs negatively, as well as for the comments that attribute this level of inferior performance to have been to work with a number expressively bigger of stakeholders in the public context.

My interpretation

A summarized preliminary interpretation, in the light of the theory, is that to deal with these opposing forces and to enable the management of expectations and demands of stakeholders, management theory offers solutions for understanding the power and political contexts, as well as successful professional managing these forces. It also provides extensive theory and case studies for effective mapping and stakeholder management and effective organisational communication.

Open-ended questions

Do you agree with this interpretation? If not, why not?

In practical terms, what could be done to lead the public SSC to overcome the political interference and the huge number of stakeholders? 


\section{2) Key findings of the quantitative part of the survey}

In my thesis, I am proposing the conceptual model shown in Figure 1 below. It has been tested empirically to answer the research question "To what extent does factors such as culture, leadership, resources and readiness for change, influence on service excellence, market orientation and performance of Shared Services Centres?"

Fïgure 1 Conceptual Model of Factors Influencing SSC Performance

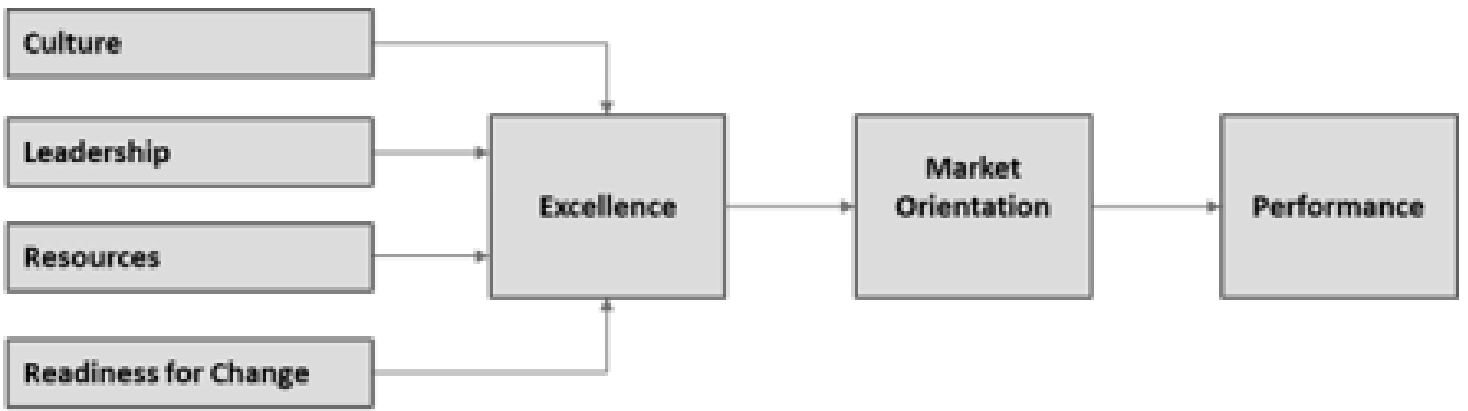

The results of quantitative research (extracted by multivariate data analysis) confirmed that there is a positive relationship between the elements (variables) of the proposed model, validating the model. However, the relationship between Excellence and Market Orientation proved to be much weaker than the relationship between Excellence and Performance. Also, the relationship between Market Orientation and Performance was weak. In both cases, for public and private SSCs.

My interpretation

An initial interpretation could be that the degree of market orientation (customeroriented SSC) does not influence much on the SSC performance and that the level of excellence is more crucial in this.

Open-ended questions

What is your opinion about this?

In practical terms, what could be done to lead the public SSC to be more customeroriented? 
Overall, considering the qualitative and quantitative survey parts, in your opinion, which other knowledge generated could be turned into practical actions to improve SSCs?

\section{Example 3}

\section{Second and final round of the Action Learning Set (ALS)}

Overall, there was agreement with the majority.of the preliminary interpretations, but with important questions and 'new readings' about the context of Shared Services Centres in the public sector that I had not thought of them. All this is helping in my development, and I am grateful.

As the primary objective of our ALS is transforming the knowledge generated by the doctoral thesis research on possible actions for improvement of SSCs (focusing on public sector), I analysed the preliminary findings again, reflecting on your questions and 'new readings' of the reality presented by you. In this way, I reached six measures that the SSCs of the public sector can adopt to perfect its service operations (the qualitative part of the survey) and gave them the next titles: 1 . Create its own service culture; 2 . Steering by its customers (client focus); 3. Develop an entrepreneurial leadership; 4. Bring some people from the industry to the public SSC; 5. Public SSC as a State policy; and 6. Organisational resilience. The established sequence reflects the classification for a bigger number of comments and quotations of the ALS.

These measures are presented individually following (I tried to summarize the content and structured as Likert scale to be quicker to respond, and to optimize your time) and I ask that you, please, express your opinion on each one of them (from strongly disagree to strongly agree) and make your comments, criticisms, and suggestions, so you will help me to check whether the measures make sense and improve them. Immediately after, two other measures are proposed concerning the quantitative part of the survey.

\section{A) As for the qualitative part of the survey}

Certainly, the measures proposed following will depend on favourable context for its adoption, but each one of them in some extension is already adopted in public SSCs of countries like Canada, the USA, and the UK.

1. Create its own service culture 
It should be allowed to an SSC have its own culture, oriented to the delivery of service to the customer with high employee engagement; combining with creative responses, the excellence in service with the necessity of fulfilment of the legislation and the limitations inherent in the civil service. The SSC should also have mechanisms to 'chargeback,' charging for their services and thus having the resources to invest.

(Please express your opinion by including an " $\mathrm{X}$ " in the box of your choice.)

\begin{tabular}{|l|l|l|l|c|}
\hline $\begin{array}{c}\text { Strongly } \\
\text { disagree }\end{array}$ & Disagree & Neutral & Agree & $\begin{array}{c}\text { Strongly } \\
\text { agree }\end{array}$ \\
\hline & & & & \\
\hline \multicolumn{5}{|c|}{ Please enter your comments in the box below. } \\
\hline
\end{tabular}

The focus of the client and his active participation in the direction of the SSC.

\section{Steering by its customers (client focus)}

An SSC must, by definition, be directed by its customers, and this direction level is typically more strategic (not in the daily management of the SSC), through a Council or similar mechanism. The public SSC will naturally be 'more competitive' if guided and directed by its customers (in the words of one of the members of the ALS to 'Do what the customer wants/needs'). Note: competitive here has the meaning to respond with effectiveness the needs of its customers.

(Please express your opinion by including an " $\mathrm{X}$ " in the box of your choice.)

\begin{tabular}{|l|l|l|l|c|}
\hline $\begin{array}{c}\text { Strongly } \\
\text { disagree }\end{array}$ & Disagree & Neutral & Agree & $\begin{array}{c}\text { Strongly } \\
\text { agree }\end{array}$ \\
\hline & & & & \\
\hline \multicolumn{5}{|c|}{ Please enter your comments in the box below. } \\
\hline
\end{tabular}

Leadership.

\section{Develop an entrepreneurial leadership}

An entrepreneurial leadership can revolutionise a lenient, accommodated SSC, even with the limitations arising from bureaucracy, scope defined by law, and limitations related to staff. This can lead to the transformation of the attitude of the SSC staff and positively affect service quality.

(Please express your opinion by including an " $\mathrm{X}$ " in the box of your choice.) 


\begin{tabular}{|c|c|c|c|c|}
\hline $\begin{array}{c}\text { Strongly } \\
\text { disagree }\end{array}$ & Disagree & Neutral & Agree & $\begin{array}{c}\text { Strongly } \\
\text { agree }\end{array}$ \\
\hline & & & & \\
\hline \multicolumn{5}{|c|}{ Please enter your comments in the box below. } \\
\hline \multicolumn{3}{|c|}{}
\end{tabular}

There is already a considerable number of experienced professionals in best practices of SSC in the private sector in many countries. Why not use their apprenticeship as a reference for the cultural change (role model) in the public sector SSCs that need this change?

4. Bring some people from the industry to the public SSC

People with the right skills to promote high-performance SSCs could be brought from the private sector to the SSCs of the public sector that need. This could occur on a temporary basis, by project, or other similar initiatives. Public-private partnerships could also provide the legal means for the establishment of SSCs that can count on professionals from the private sector; they can disseminate best practice and form a culture that favours acting as a client-oriented service unit. A member of the ALS commented that the public sector would need to develop mechanisms to identify the right people for doing that.

(Please express your opinion by including an " $\mathrm{X}$ " in the box of your choice.)

\begin{tabular}{|c|c|c|c|c|}
\hline $\begin{array}{c}\text { Strongly } \\
\text { disagree }\end{array}$ & Disagree & Neutral & Agree & $\begin{array}{c}\text { Strongly } \\
\text { agree }\end{array}$ \\
\hline & & & & \\
\hline
\end{tabular}

Please enter your comments in the box below.

A recurrent problem in the SSCs of the public sector in many countries, but not all, is the political interference in the exchange of governments, mainly from different parties. A measure to mitigate this risk is to turn the SSCs (together with other public services) into politics of State and not of government.

\section{Public SSC as a State policy}

The SSC should be part of a State policy, not a government policy, not to be discontinued (or severely impacted) when there is a change of government. An ALS member informs that in the US, for example, the level of this kind of political interference in federal SSCs, practically does not exist.

(Please express your opinion by including an " $\mathrm{X}$ " in the box of your choice.) 


\begin{tabular}{|l|c|c|c|c|}
\hline $\begin{array}{c}\text { Strongly } \\
\text { disagree }\end{array}$ & Disagree & Neutral & Agree & $\begin{array}{c}\text { Strongly } \\
\text { agree }\end{array}$ \\
\hline & & & & \\
\hline \multicolumn{5}{|c|}{ Please enter your comments in the box below. } \\
\hline
\end{tabular}

Create mechanisms that can 'shield' the SSCs to the extent possible against unwanted harmful political interference.

\section{Organisational resilience}

Public SSCs should have the ability to quickly adapt, respond and continue to provide high-quality services to its customers, regardless of possible changes brought about in the political context. (Shield the SSC against political interference.)

(Please express your opinion by including an " $\mathrm{X}$ " in the box of your choice.)

\begin{tabular}{|c|c|c|c|c|}
\hline $\begin{array}{c}\text { Strongly } \\
\text { disagree }\end{array}$ & Disagree & Neutral & Agree & $\begin{array}{c}\text { Strongly } \\
\text { agree }\end{array}$ \\
\hline & & & \\
\hline \multicolumn{5}{|c|}{ Please enter your comments in the box below. } \\
\hline
\end{tabular}

\section{B) As for the quantitative part of the survey}

In the quantitative part of the survey, a comment caught my attention as for those points on Market orientation and Excellence, apparently conflicting regarding to their relationship with the SSC performance, raised in the preliminary analysis. One of the ALS members commented that this is because of the conceptual model has captured the current view of respondents and not the desired view for public SSC. From this point of view, it is argued that public SSCs should, for sure, structure their service more and more from the customer focus.

1. It is desirable that the SSCs of the public sector are 'market-oriented' in their practices, that is, the design of its service operation takes into account primarily the needs of its customers (in addition to other stakeholders). Note: 'market' understood here as the external environment to the SSC ('outside-in' view).

(Please express your opinion by including an " $\mathrm{X}$ " in the box of your choice.) 


\begin{tabular}{|l|c|c|c|c|}
\hline $\begin{array}{c}\text { Strongly } \\
\text { disagree }\end{array}$ & Disagree & Neutral & Agree & $\begin{array}{c}\text { Strongly } \\
\text { agree }\end{array}$ \\
\hline & & & \\
\hline \multicolumn{5}{|c|}{ Please enter your comments in the box below. } \\
\hline \multicolumn{3}{|c|}{}
\end{tabular}

It was also suggested by the ALS that the conceptual model, reflected in the questionnaire attached to the email, could be transformed into part of a diagnosis for SSCs. This would help SSC managers to verify their degree of alignment with the best practices for this kind of service operation.

2. The conceptual model of this research (reflected in detail in the questionnaire attached to the email), transformed into a diagnostic tool could be useful for an internal SSC assessment (managers and employees) and external (customers and stakeholders), providing a comparative analysis of perceptions, and resulting improvement actions.

(Please express your opinion by including an " $\mathrm{X}$ " in the box of your choice.)

\begin{tabular}{|c|c|c|c|c|}
\hline $\begin{array}{c}\text { Strongly } \\
\text { disagree }\end{array}$ & Disagree & Neutral & Agree & $\begin{array}{c}\text { Strongly } \\
\text { agree }\end{array}$ \\
\hline & & & \\
\hline \multicolumn{5}{|c|}{ Please enter your comments in the box below. } \\
\hline \multicolumn{3}{|c|}{} \\
\hline
\end{tabular}

Any other suggestions, criticism or recommendation for the work as a whole?

I take this opportunity to thank you once again for your time and dedication.

I look forward to your reply. If you can send it up to 0ct 02, 2016, or earlier, I will appreciate very much.

Best regards,

Cicero Ferreira 